Supporting Information for

\title{
Construction of Spironaphthalenones via Gold-Catalyzed Intramolecular Dearomatization Reaction of $\beta$-Naphthol Derivatives
}

\author{
Lu Ding, ${ }^{\ddagger}$ Wen-Ting Wu, ${ }^{\dagger}$ Liming Zhang, ${ }^{* \S}$ and Shu-Li You* ${ }^{\star \ddagger}$
}

\begin{abstract}
† State Key Laboratory of Organometallic Chemistry, Center for Excellence in Molecular Synthesis, Shanghai Institute of Organic Chemistry, Chinese Academy of Sciences, 345 Lingling Lu, Shanghai 200032, China

ॠSchool of Physical Science and Technology, ShanghaiTech University, 100 Haike Road, Shanghai 201210, China

${ }^{\S}$ Department of Chemistry \& Biochemistry, University of California, Santa Barbara, California 93106, USA

E-mail: zhang@chem.ucsb.edu; slyou@sioc.ac.cn
\end{abstract}

\section{Table of Contents}

1. General methods

S2

2. Experimental details and characterization data

S3-S24

2.1. General procedure for the synthesis of naphthol derivatives

S3-S12

2.2. General procedure for gold-catalyzed intramolecular dearomatization reaction of $\beta$-naphthol derivatives

2.3. Transformation of products

3. References

S24

4. X-Ray crystal data of $\mathbf{2 a}$

S24-S32

5. Copies of NMR spectra and HPLC chromatographs

S33-S119 


\section{General Methods.}

Unless stated otherwise, all reactions were carried out in open-flask. All solvents were not purified and dried prior to use.

${ }^{1} \mathrm{H}$ NMR spectra were recorded on a Varian $(400 \mathrm{MHz})$ or Agilent instrument (400 $\mathrm{MHz}$ or $600 \mathrm{MHz}$ ) and internally referenced to tetramethylsilane signal or residual solvent signals. ${ }^{13} \mathrm{C}$ NMR spectra were recorded on a Varian $(100 \mathrm{MHz})$ or Agilent instrument $(100 \mathrm{MHz})$ and internally referenced to residual solvent signals. Data for ${ }^{1} \mathrm{H}$ NMR are recorded as follows: chemical shift $(\delta, \mathrm{ppm})$, multiplicity $(\mathrm{s}=$ singlet, $\mathrm{d}=$ doublet, $\mathrm{t}=$ triplet, $\mathrm{m}=$ multiplet or unresolved, $\mathrm{brs}=$ broad singlet, coupling constant (s) in $\mathrm{Hz}$, integration). Data for ${ }^{13} \mathrm{C} \mathrm{NMR}$ are reported in terms of chemical shift $(\delta$, ppm). The mass analyzer type used for the HRMS measurements is TOF. Oil bath is the heat source for all of our reactions that require heating.

The 1-aminoethyl-2-naphthol derivatives S1 were prepared according to the reported procedures. ${ }^{1,2}$ 


\section{Experimental details and characterization data}

\subsection{General procedure for the synthesis of naphthol derivatives (1a-1r)}

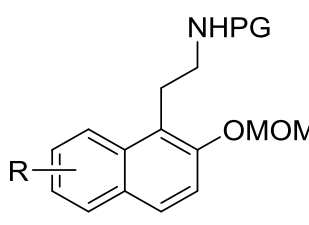

s1

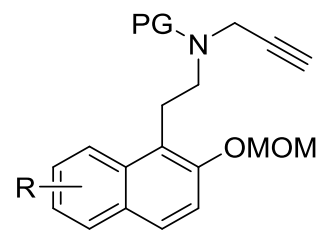

S2

Propargyl bromide (1.1 equiv) was added to a solution of $\mathbf{S 1} 1^{1,2}$ (1.0 equiv) and $\mathrm{K}_{2} \mathrm{CO}_{3}(2.0$ equiv) in $\mathrm{DMF}(0.3 \mathrm{M})$ at room temperature. After the reaction was complete (monitored by TLC), the crude reaction mixture was quenched by water and extracted with EtOAc. The organic layer was washed with brine, dried over anhydrous $\mathrm{Na}_{2} \mathrm{SO}_{4}$ and filtered. After the solvent was concentrated under reduced pressure, the crude product was purified by silica gel column chromatography $(\mathrm{PE} / \mathrm{EtOAc}=10: 1)$ to afford the desired product $\mathbf{S 2}$.

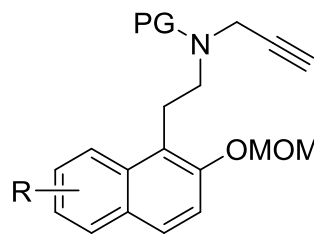

S2

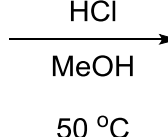

$50{ }^{\circ} \mathrm{C}$<smiles>[R][M]c1ccc2ccc(O)c(CCN([R6])CC#C)c2c1</smiles>

Concentrated $\mathrm{HCl}$ solution ( 0.7 equiv) was added to a solution of $\mathbf{S 2}$ (1.0 equiv) in $\mathrm{MeOH}(0.3 \mathrm{M})$ at room temperature. Then the reaction mixture was heated at $50{ }^{\circ} \mathrm{C}$ for $12 \mathrm{~h}$. After the reaction was complete (monitored by TLC), the crude reaction mixture was quenched by saturated $\mathrm{NaHCO}_{3}$ aqueous solution. Methanol was evaporated and the mixture was extracted with EtOAc. The organic layer was washed with brine, dried over anhydrous $\mathrm{Na}_{2} \mathrm{SO}_{4}$ and filtered. After the solvent was concentrated under reduced pressure, the crude product was purified by silica gel column chromatography $(\mathrm{PE} / \mathrm{EtOAc}=10: 1)$ to afford the desired product $(\mathbf{1 a}-1 \mathbf{r})$. The analytical data of the products are summarized below.<smiles>C#CCN([13CH3])CCc1c(O)ccc2ccccc12</smiles> 
1a: pale yellow solid, $4.61 \mathrm{~g}, 84 \%$ yield, m.p. $=66-67{ }^{\circ} \mathrm{C} .{ }^{1} \mathrm{H} \mathrm{NMR}\left(400 \mathrm{MHz}, \mathrm{CDCl}_{3}\right)$ $\delta 7.92(\mathrm{~d}, J=8.4 \mathrm{~Hz}, 1 \mathrm{H}), 7.74(\mathrm{~d}, J=8.4 \mathrm{~Hz}, 1 \mathrm{H}), 7.70(\mathrm{~d}, J=8.0 \mathrm{~Hz}, 2 \mathrm{H}), 7.62(\mathrm{~d}, J$ $=8.8 \mathrm{~Hz}, 1 \mathrm{H}), 7.46(\mathrm{t}, J=7.6 \mathrm{~Hz}, 1 \mathrm{H}), 7.30(\mathrm{t}, J=7.6 \mathrm{~Hz}, 1 \mathrm{H}), 7.20(\mathrm{~d}, J=8.0 \mathrm{~Hz}$, 2H), $7.11(\mathrm{~d}, J=9.2 \mathrm{~Hz}, 1 \mathrm{H}), 6.23$ (brs, 1H), 4.18 (d, $J=2.0 \mathrm{~Hz}, 2 \mathrm{H}), 3.42$ (s, 4H), 2.35 (s, 3H), 2.09 (t, $J=2.4 \mathrm{~Hz}, 1 \mathrm{H}) .{ }^{13} \mathrm{C} \mathrm{NMR}\left(100 \mathrm{MHz}, \mathrm{CDCl}_{3}\right) \delta 151.9,143.8,135.2$, 133.2, 129.6, 129.2, 128.7, 128.6, 127.7, 126.8, 123.1, 122.4, 118.1, 115.4, 77.2, 73.9, 46.3, 37.6, 24.9, 21.6; IR (film): $\mathrm{v}_{\max }\left(\mathrm{cm}^{-1}\right)=3431,3283,1659,1598,1513,1495$, $1453,1438,1399,1323,1306,1267,1210,1150,1093,1065,1035,1018,1002,956$, 901, 865, 810, 746, 704, 656, 577, 543, 424; HRMS (ESI): Exact mass calcd. for $\mathrm{C}_{22} \mathrm{H}_{22} \mathrm{NO}_{3} \mathrm{~S}\left([\mathrm{M}+\mathrm{H}]^{+}\right): 380.1315$. Found: 380.1322 .

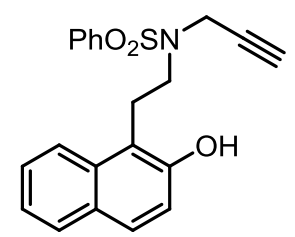

1b: pale yellow solid, $978 \mathrm{mg}$, 93\% yield, m.p. $=85-87^{\circ} \mathrm{C} .{ }^{1} \mathrm{H} \mathrm{NMR}\left(400 \mathrm{MHz}, \mathrm{CDCl}_{3}\right)$ $\delta 7.93(\mathrm{~d}, J=8.4 \mathrm{~Hz}, 1 \mathrm{H}), 7.84(\mathrm{~d}, J=7.6 \mathrm{~Hz}, 2 \mathrm{H}), 7.76(\mathrm{~d}, J=8.4 \mathrm{~Hz}, 1 \mathrm{H}), 7.65(\mathrm{~d}, J$ $=8.8 \mathrm{~Hz}, 1 \mathrm{H}), 7.55-7.43(\mathrm{~m}, 4 \mathrm{H}), 7.32(\mathrm{t}, J=7.6 \mathrm{~Hz}, 1 \mathrm{H}), 7.11(\mathrm{~d}, J=8.8 \mathrm{~Hz}, 1 \mathrm{H})$, 5.99 (brs, 1H), 4.19 (d, $J=1.6 \mathrm{~Hz}, 2 \mathrm{H}), 3.44$ (s, 4H), 2.08 (s, 1H). ${ }^{13} \mathrm{C}$ NMR (100 MHz, $\left.\mathrm{CDCl}_{3}\right) \delta 151.8,138.3,133.2,132.9,129.2,129.0,128.7,128.6,127.6,126.8,123.1$, 122.3, 118.0, 115.3, 77.0, 73.9, 46.3, 37.7, 24.9; IR (film): $\mathrm{V}_{\max }\left(\mathrm{cm}^{-1}\right)=3432,3280$, 2380, 2348, 2323, 1626, 1604, 1582, 1514, 1478, 1439, 1349, 1322, 1265, 1245, 1211, 1159, 1129, 1096, 1067, 1038, 1024, 923, 903, 861, 813, 793, 745, 730, 686, 675, 647, 598, 552, 481, 423; HRMS (ESI): Exact mass calcd. for $\mathrm{C}_{21} \mathrm{H}_{20} \mathrm{NO}_{3} \mathrm{~S}\left([\mathrm{M}+\mathrm{H}]^{+}\right)$: 366.1158. Found: 366.1165.

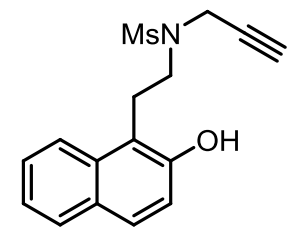

1c: pale yellow solid, $934 \mathrm{mg}, 83 \%$ yield, m.p. $=109-110{ }^{\circ} \mathrm{C} .{ }^{1} \mathrm{H}$ NMR $(400 \mathrm{MHz}$, $\left.\mathrm{CDCl}_{3}\right) \delta 7.96(\mathrm{~d}, J=8.8 \mathrm{~Hz}, 1 \mathrm{H}), 7.77(\mathrm{~d}, J=8.0 \mathrm{~Hz}, 1 \mathrm{H}), 7.66(\mathrm{~d}, J=8.8 \mathrm{~Hz}, 1 \mathrm{H})$, $7.51(\mathrm{t}, J=7.2 \mathrm{~Hz}, 1 \mathrm{H}), 7.34$ (t, $J=7.2 \mathrm{~Hz}, 1 \mathrm{H}), 7.10$ (d, $J=8.8 \mathrm{~Hz}, 1 \mathrm{H}), 5.88$ (brs, 
$1 \mathrm{H}), 4.12(\mathrm{~d}, J=2.4 \mathrm{~Hz}, 2 \mathrm{H}), 3.53(\mathrm{dd}, J=10.4,6.4 \mathrm{~Hz}, 2 \mathrm{H}), 3.41(\mathrm{dd}, J=8.8,5.2 \mathrm{~Hz}$, 2H), $2.92(\mathrm{~s}, 3 \mathrm{H}), 2.35(\mathrm{t}, J=2.4 \mathrm{~Hz}, 1 \mathrm{H}) .{ }^{13} \mathrm{C} \mathrm{NMR}\left(100 \mathrm{MHz}, \mathrm{CDCl}_{3}\right) \delta 151.7,133.1$, 129.2, 128.73, 128.70, 126.9, 123.2, 122.3, 117.9, 115.3, 77.6, 74.2, 46.5, 37.9, 37.4, 25.0; IR (film): $V_{\max }\left(\mathrm{cm}^{-1}\right)=3493,3277,1627,1607,1584,1514,1479,1461,1438$, 1428, 1380, 1355, 1336, 1318, 1265, 1211, 1173, 1141, 1092, 1060, 1031, 1006, 978, $909,864,816,797,778,763,743,694,672,624,582,553,509,475,438,420$; HRMS (ESI): Exact mass calcd. for $\mathrm{C}_{16} \mathrm{H}_{21} \mathrm{~N}_{2} \mathrm{O}_{3} \mathrm{~S}\left(\left[\mathrm{M}+\mathrm{NH}_{4}\right]^{+}\right)$: 321.1267 . Found: 321.1279 .

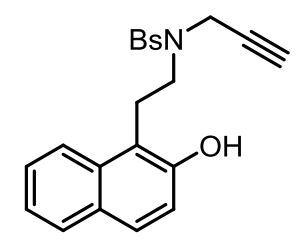

1d: brown solid, $1.65 \mathrm{~g}, 93 \%$ yield, m.p. $=68-70{ }^{\circ} \mathrm{C} .{ }^{1} \mathrm{H} \mathrm{NMR}\left(400 \mathrm{MHz}, \mathrm{CDCl}_{3}\right) \delta$ $7.90(\mathrm{~d}, J=8.4 \mathrm{~Hz}, 1 \mathrm{H}), 7.74(\mathrm{~d}, J=8.0 \mathrm{~Hz}, 1 \mathrm{H}), 7.64-7.60(\mathrm{~m}, 3 \mathrm{H}), 7.50-7.45(\mathrm{~m}, 3 \mathrm{H})$, $7.31(\mathrm{t}, J=7.2 \mathrm{~Hz}, 1 \mathrm{H}), 7.06$ (d, $J=8.4 \mathrm{~Hz}, 1 \mathrm{H}), 6.07$ (brs, 1H), 4.22 (s, 2H), 3.45-3.41 (m, 4H), 2.11 (s, 1H). ${ }^{13} \mathrm{C} \mathrm{NMR}\left(100 \mathrm{MHz}, \mathrm{CDCl}_{3}\right) \delta 151.7,137.4,133.2,132.1,129.2$, $129.1,128.8,128.7,127.9,126.9,123.2,122.3,117.9,115.4,76.8,74.2,46.1,37.3$, 24.5; IR (film): $V_{\max }\left(\mathrm{cm}^{-1}\right)=3439,3286,2925,1626,1602,1573,1513,1470,1437$, 1389, 1328, 1266, 1207, 1152, 1095, 1067, 1034, 1006, 901, 866, 811, 749, 669, 603, 569, 554, 536, 480, 419; HRMS (ESI): Exact mass calcd. for $\mathrm{C}_{21} \mathrm{H}_{19} \mathrm{BrNO}_{3} \mathrm{~S}\left([\mathrm{M}+\mathrm{H}]^{+}\right)$: 444.0264. Found: 444.0250.

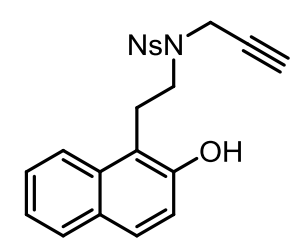

1e: yellow solid, 1.30 g, 93\% yield, m.p. $=90-92{ }^{\circ} \mathrm{C} .{ }^{1} \mathrm{H} \mathrm{NMR}\left(400 \mathrm{MHz}, \mathrm{CDCl}_{3}\right) \delta$ $8.17(\mathrm{~d}, J=8.8 \mathrm{~Hz}, 2 \mathrm{H}), 7.92-7.88(\mathrm{~m}, 3 \mathrm{H}), 7.73(\mathrm{~d}, J=8.0 \mathrm{~Hz}, 1 \mathrm{H}), 7.63$ (d, $J=8.4$ $\mathrm{Hz}, 1 \mathrm{H}), 7.49$ (t, $J=8.4 \mathrm{~Hz}, 1 \mathrm{H}), 7.32$ (t, $J=8.0 \mathrm{~Hz}, 1 \mathrm{H}), 7.03$ (d, $J=8.8 \mathrm{~Hz}, 1 \mathrm{H}), 5.53$ (brs, $1 \mathrm{H}), 4.31(\mathrm{~d}, J=2.4 \mathrm{~Hz}, 2 \mathrm{H}), 3.52$ (dd, $J=9.6,6.8 \mathrm{~Hz}, 2 \mathrm{H}), 3.40$ (dd, $J=8.4,5.6$ $\mathrm{Hz}, 2 \mathrm{H}), 2.14(\mathrm{t}, J=2.4 \mathrm{~Hz}, 1 \mathrm{H}) .{ }^{13} \mathrm{C} \mathrm{NMR}\left(100 \mathrm{MHz}, \mathrm{CDCl}_{3}\right) \delta 151.5,149.8,144.3$, 133.0, 129.2, 128.8, 128.7, 127.0, 123.9, 123.4, 122.1, 117.7, 115.3, 76.6, 74.4, 46.0, 37.0, 24.1; IR (film): $V_{\max }\left(\mathrm{cm}^{-1}\right)=3437,3288,1630,1608,1586,1527,1479,1454$, 
$1432,1401,1346,1322,1303,1282,1264,1208,1147,1100,1080,1031,1001,976$, $952,922,896,872,851,803,776,759,739,716,679,655,608,589,562,520,472$, 456, 432; HRMS (ESI): Exact mass calcd. for $\mathrm{C}_{21} \mathrm{H}_{19} \mathrm{~N}_{2} \mathrm{O}_{5} \mathrm{~S}\left([\mathrm{M}+\mathrm{H}]^{+}\right)$: 411.1009 . Found: 411.1007.

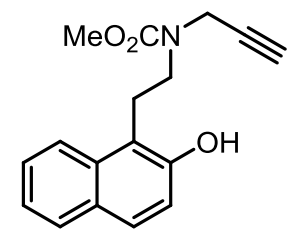

1f: pale white solid, $677 \mathrm{mg}, 88 \%$ yield, m.p. $=66-67{ }^{\circ} \mathrm{C} .{ }^{1} \mathrm{H}$ NMR $(400 \mathrm{MHz}$, $\left.\left(\mathrm{CD}_{3}\right)_{2} \mathrm{CO}\right) \delta 8.62$ (brs, 1H), $8.12(\mathrm{~d}, J=34.4 \mathrm{~Hz}, 1 \mathrm{H}), 7.79(\mathrm{~d}, J=8.0 \mathrm{~Hz}, 1 \mathrm{H}), 7.68$ $(\mathrm{d}, J=8.8 \mathrm{~Hz}, 1 \mathrm{H}), 7.49$ (t, $J=7.6 \mathrm{~Hz}, 1 \mathrm{H}), 7.30$ (t, $J=7.2 \mathrm{~Hz}, 1 \mathrm{H}), 7.22(\mathrm{~d}, J=8.8$ $\mathrm{Hz}, 1 \mathrm{H}), 4.15$ (brs, 2H), 3.73-3.61 (m, 5H), 3.43-3.39 (m, 2H), 2.75 (brs, $1 \mathrm{H}) .{ }^{13} \mathrm{C}$ NMR (100 MHz, $\left.\left(\mathrm{CD}_{3}\right)_{2} \mathrm{CO}\right) \delta 155.9,152.7,133.8,129.0,128.5,128.0,126.3,122.5,117.7$, 116.4, 79.8, 72.3, 52.1, 46.8, 45.5, 36.3, 23.7; IR (film): $\mathrm{v}_{\max }\left(\mathrm{cm}^{-1}\right)=3308,3284,1673$, $1623,1601,1579,1511,1475,1434,1402,1356,1316,1255,1169,1149,1122,1053$, $1019,980,943,929,915,865,837,815,773,745,696,660,635,571,533,491,477$, 424; HRMS (ESI): Exact mass calcd. for $\mathrm{C}_{17} \mathrm{H}_{18} \mathrm{NO}_{3}\left([\mathrm{M}+\mathrm{H}]^{+}\right)$: 284.1281. Found: 284.1293.

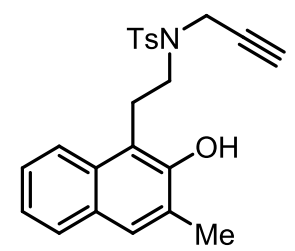

1g: pale white solid, $1.90 \mathrm{~g}, 91 \%$ yield, m.p. $=60-62{ }^{\circ} \mathrm{C} .{ }^{1} \mathrm{H}$ NMR $\left(400 \mathrm{MHz}, \mathrm{CDCl}_{3}\right)$ $\delta 7.86(\mathrm{~d}, J=8.4 \mathrm{~Hz}, 1 \mathrm{H}), 7.73-7.68(\mathrm{~m}, 3 \mathrm{H}), 7.52(\mathrm{~s}, 1 \mathrm{H}), 7.42(\mathrm{t}, J=7.2 \mathrm{~Hz}, 1 \mathrm{H})$, 7.30 (t, $J=7.2 \mathrm{~Hz}, 1 \mathrm{H}), 7.24$ (d, $J=7.2 \mathrm{~Hz}, 2 \mathrm{H}), 5.89$ (brs, 1H), 4.15 (s, 2H), 3.41 (s, 4H), $2.43(\mathrm{~s}, 3 \mathrm{H}), 2.38(\mathrm{~s}, 3 \mathrm{H}), 2.12(\mathrm{~s}, 1 \mathrm{H}) .{ }^{13} \mathrm{C} \mathrm{NMR}\left(100 \mathrm{MHz}, \mathrm{CDCl}_{3}\right) \delta$ 151.3, $143.8,135.2$, 131.9, 129.6, 129.1, 128.3, 127.9, 127.7, 126.3, 125.8, 123.2, 122.1, 114.7, 77.3, 73.8, 46.4, 38.0, 25.6, 21.6, 17.1; IR (film): $\mathrm{V}_{\max }\left(\mathrm{cm}^{-1}\right)=3489,3283,1629,1598$, $1507,1447,1399,1376,1328,1306,1288,1251,1205,1180,1150,1093,1032,1006$, 909, 883, 852, 812, 745, 701, 659, 578, 542, 450, 432; HRMS (ESI): Exact mass calcd. for $\mathrm{C}_{23} \mathrm{H}_{24} \mathrm{NO}_{3} \mathrm{~S}\left([\mathrm{M}+\mathrm{H}]^{+}\right)$: 394.1471. Found: 394.1480 . 


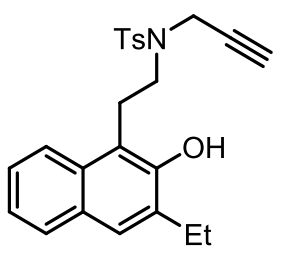

1h: pale white solid, $1.61 \mathrm{~g}, 86 \%$ yield, m.p. $=67-69{ }^{\circ} \mathrm{C} .{ }^{1} \mathrm{H} \mathrm{NMR}\left(400 \mathrm{MHz}, \mathrm{CDCl}_{3}\right)$ $\delta 7.85(\mathrm{~d}, J=8.4 \mathrm{~Hz}, 1 \mathrm{H}), 7.71-7.69(\mathrm{~m}, 3 \mathrm{H}), 7.50(\mathrm{~s}, 1 \mathrm{H}), 7.41(\mathrm{t}, J=7.2 \mathrm{~Hz}, 1 \mathrm{H})$, $7.28(\mathrm{t}, J=7.2 \mathrm{~Hz}, 1 \mathrm{H}), 7.20$ (d, $J=7.2 \mathrm{~Hz}, 2 \mathrm{H}), 6.06$ (brs, 1H), 4.12 (s, 2H), 3.41 (s, 4H), $2.79(\mathrm{dd}, J=14.4,7.2 \mathrm{~Hz}, 2 \mathrm{H}), 2.34(\mathrm{~s}, 3 \mathrm{H}), 2.11(\mathrm{~s}, 1 \mathrm{H}), 1.32$ (t, $J=7.2 \mathrm{~Hz}, 3 \mathrm{H})$. ${ }^{13} \mathrm{C}$ NMR (100 MHz, $\left.\mathrm{CDCl}_{3}\right) \delta 151.1,143.9,135.2,132.3,131.8,129.6,129.2,128.2$, $127.7,126.5,125.9,123.2,122.1,114.9,77.3,74.0,46.5,38.0,25.6,23.9,21.6,13.9$; IR (film): $\mathrm{V}_{\max }\left(\mathrm{cm}^{-1}\right)=3529,3269,3060,2976,2907,2878,2120,1630,1597,1580$, 1504, 1441, 1404, 1382, 1342, 1324, 1279, 1254, 1235, 1201, 1174, 1148, 1101, 1068, 1049, 1006, 939, 906, 891, 852, 805, 746, 708, 683, 659, 618, 577, 539, 522, 497, 441; HRMS (ESI): Exact mass calcd. for $\mathrm{C}_{24} \mathrm{H}_{26} \mathrm{NO}_{3} \mathrm{~S}\left([\mathrm{M}+\mathrm{H}]^{+}\right)$: 408.1628. Found: 408.1637.

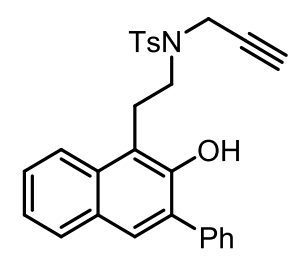

1i: pale white solid, 2.53 g, 94\% yield, m.p. $=81-83{ }^{\circ} \mathrm{C} .{ }^{1} \mathrm{H}$ NMR $\left(400 \mathrm{MHz}, \mathrm{CDCl}_{3}\right) \delta$ $8.01(\mathrm{~d}, J=8.4 \mathrm{~Hz}, 1 \mathrm{H}), 7.77(\mathrm{~d}, J=8.0 \mathrm{~Hz}, 1 \mathrm{H}), 7.70(\mathrm{~d}, J=8.0 \mathrm{~Hz}, 2 \mathrm{H}), 7.64(\mathrm{~s}, 1 \mathrm{H})$, 7.53-7.44 (m, 6H), 7.35 (t, $J=7.2 \mathrm{~Hz}, 1 \mathrm{H}), 7.20$ (d, $J=8.0 \mathrm{~Hz}, 2 \mathrm{H}), 5.58$ (brs, 1H), 4.27 (s, 2H), 3.48 (s, 4H), 2.36 (s, 3H), 2.10 (s, 1H). $\left.{ }^{13} \mathrm{C} \mathrm{NMR} \mathrm{(100} \mathrm{MHz,} \mathrm{CDCl}_{3}\right) \delta$ 148.7, 143.4, 136.9, 135.9, 132.9, 130.1, 129.4, 129.3, 128.9, 128.6, 128.4, 127.6, 126.9, 123.6, 122.6, 116.3, 77.3, 73.6, 46.0, 37.1, 25.0, 21.5; IR (film): $v_{\max }\left(\mathrm{cm}^{-1}\right)=3537$, $3278,1731,1629,1597,1580,1501,1430,1372,1347,1329,1306,1281,1242,1212$, 1198, 1158, 1094, 1042, 1004, 976, 947, 930, 895, 814, 766, 749, 706, 657, 575, 555, 542, 514, 495, 463, 422; HRMS (ESI): Exact mass calcd. for $\mathrm{C}_{28} \mathrm{H}_{26} \mathrm{NO}_{3} \mathrm{~S}\left([\mathrm{M}+\mathrm{H}]^{+}\right)$: 456.1628. Found: 456.1633. 


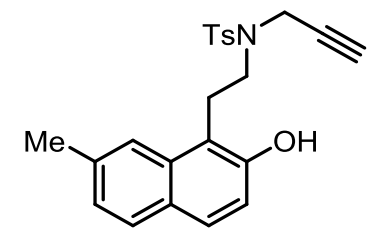

1j: pale white solid, $2.90 \mathrm{~g}, 86 \%$ yield, m.p. $=84-86{ }^{\circ} \mathrm{C} .{ }^{1} \mathrm{H}$ NMR $\left(400 \mathrm{MHz}, \mathrm{CDCl}_{3}\right)$ $\delta 7.72-7.70(\mathrm{~m}, 3 \mathrm{H}), 7.64(\mathrm{~d}, J=8.4 \mathrm{~Hz}, 1 \mathrm{H}), 7.58(\mathrm{~d}, J=8.4 \mathrm{~Hz}, 1 \mathrm{H}), 7.21(\mathrm{~d}, J=7.6$ $\mathrm{Hz}, 2 \mathrm{H}), 7.15$ (d, $J=8.0 \mathrm{~Hz}, 1 \mathrm{H}), 7.03$ (d, $J=8.8 \mathrm{~Hz}, 1 \mathrm{H}), 6.03$ (brs, 1H), 4.20 (s, 2H), 3.42-3.41 (m, 4H), $2.50(\mathrm{~s}, 3 \mathrm{H}), 2.36(\mathrm{~s}, 3 \mathrm{H}), 2.11(\mathrm{~s}, 1 \mathrm{H}) .{ }^{13} \mathrm{C} \mathrm{NMR}\left(100 \mathrm{MHz}, \mathrm{CDCl}_{3}\right)$ $\delta 151.9,143.7,136.4,135.3,133.3,129.5,128.5,128.3,127.7,127.4,125.3,121.6$, 117.1, 114.8, 77.2, 73.8, 46.2, 37.6, 24.8, 22.2, 21.5; IR (film): $\mathrm{v}_{\max }\left(\mathrm{cm}^{-1}\right)=3428,3308$, $3289,1731,1633,1583,1514,1496,1455,1437,1322,1305,1270,1241,1151,1097$, $1060,1041,1019,1004,952,916,894,831,814,794,754,721,658,635,577,547$, 504, 432; HRMS (ESI): Exact mass calcd. for $\mathrm{C}_{23} \mathrm{H}_{24} \mathrm{NO}_{3} \mathrm{~S}\left([\mathrm{M}+\mathrm{H}]^{+}\right)$: 394.1471. Found: 394.1483.<smiles>C#CCN([13CH3])CCc1c(O)ccc2ccc(OC)cc12</smiles>

1k: pale white solid, $423 \mathrm{mg}, 97 \%$ yield, m.p. $=70-72{ }^{\circ} \mathrm{C} .{ }^{1} \mathrm{H} \mathrm{NMR}\left(400 \mathrm{MHz}, \mathrm{CDCl}_{3}\right)$ $\delta 7.69(\mathrm{~d}, J=8.0 \mathrm{~Hz}, 2 \mathrm{H}), 7.62(\mathrm{~d}, J=8.8 \mathrm{~Hz}, 1 \mathrm{H}), 7.52(\mathrm{~d}, J=8.8 \mathrm{~Hz}, 1 \mathrm{H}), 7.32(\mathrm{~s}$, $1 \mathrm{H}), 7.19(\mathrm{~d}, J=8.0 \mathrm{~Hz}, 2 \mathrm{H}), 6.98(\mathrm{~d}, J=8.4 \mathrm{~Hz}, 1 \mathrm{H}), 6.92(\mathrm{~d}, J=8.8 \mathrm{~Hz}, 1 \mathrm{H}), 6.09$ (brs, 1H), $4.18(\mathrm{~s}, 2 \mathrm{H}), 3.96(\mathrm{~s}, 3 \mathrm{H}), 3.45-3.38(\mathrm{~m}, 4 \mathrm{H}), 2.34(\mathrm{~s}, 3 \mathrm{H}), 2.08(\mathrm{~s}, 1 \mathrm{H}) .{ }^{13} \mathrm{C}$ NMR (100 MHz, $\left.\mathrm{CDCl}_{3}\right) \delta 158.7,152.2,143.7,135.5,134.7,130.1,129.6,128.2,127.5$, 124.5, 115.6, 115.2, 114.7, 101.5, 77.3, 73.8, 55.5, 46.0, 37.5, 25.2, 21.5; IR (film): $v_{\max }$ $\left(\mathrm{cm}^{-1}\right)=3384,3260,1626,1588,1514,1463,1450,1434,1379,1348,1322,1305$, $1281,1263,1247,1217,1183,1156,1138,1098,1044,1030,1007,967,930,895,832$, 802, 786, 752, 720, 662, 617, 580, 550, 486, 465, 442; HRMS (ESI): Exact mass calcd. for $\mathrm{C}_{23} \mathrm{H}_{24} \mathrm{NO}_{4} \mathrm{~S}\left([\mathrm{M}+\mathrm{H}]^{+}\right)$: 410.1421. Found: 410.1429 . 


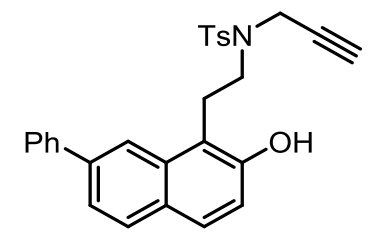

11: pale yellow solid, 2.27 g, 96\% yield, m.p. $=64-65{ }^{\circ} \mathrm{C} .{ }^{1} \mathrm{H}$ NMR $\left(400 \mathrm{MHz}, \mathrm{CDCl}_{3}\right)$ $\delta 8.14(\mathrm{~s}, 1 \mathrm{H}), 7.78(\mathrm{~d}, J=8.4 \mathrm{~Hz}, 1 \mathrm{H}), 7.73(\mathrm{~d}, J=7.2 \mathrm{~Hz}, 2 \mathrm{H}), 7.67$ (d, $J=8.4 \mathrm{~Hz}$, 2H), $7.61(\mathrm{~d}, J=8.8 \mathrm{~Hz}, 1 \mathrm{H}), 7.57-7.54(\mathrm{~m}, 1 \mathrm{H}), 7.45$ (t, $J=8.0 \mathrm{~Hz}, 2 \mathrm{H}), 7.34$ (t, $J=$ $7.6 \mathrm{~Hz}, 1 \mathrm{H}), 7.13$ (d, $J=8.4 \mathrm{~Hz}, 2 \mathrm{H}), 7.11$ (d, $J=8.8 \mathrm{~Hz}, 1 \mathrm{H}), 6.29$ (brs, 1H), 4.21 (d, $J=2.4 \mathrm{~Hz}, 2 \mathrm{H}), 3.47(\mathrm{~s}, 4 \mathrm{H}), 2.30(\mathrm{~s}, 3 \mathrm{H}), 2.01(\mathrm{t}, J=2.4 \mathrm{~Hz}, 1 \mathrm{H}) .{ }^{13} \mathrm{C} \mathrm{NMR}(100 \mathrm{MHz}$, $\left.\mathrm{CDCl}_{3}\right) \delta 152.3,143.8,141.5,139.4,135.2,133.5,129.6,129.2,128.8,128.4,128.3$, 127.7, 127.6, 127.4, 122.8, 120.6, 118.2, 115.8, 77.2, 74.1, 46.2, 37.6, 24.8, 21.5; IR (film): $V_{\max }\left(\mathrm{cm}^{-1}\right)=3431,3286,2144,2093,1625,1598,1577,1514,1492,1449,1434$, 1376, 1342, 1325, 1286, 1247, 1186, 1150, 1096, 1041, 1007, 906, 877, 837, 812, 754, 722, 697, 656, 580, 544, 442, 410; HRMS (ESI): Exact mass calcd. for $\mathrm{C}_{28} \mathrm{H}_{26} \mathrm{NO}_{3} \mathrm{~S}$ $\left([\mathrm{M}+\mathrm{H}]^{+}\right): 456.1628$. Found: 456.1634 .

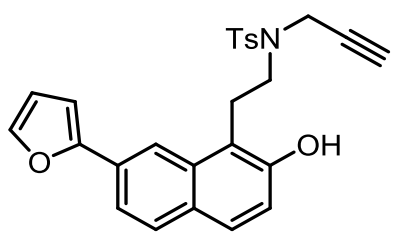

1m: brown solid, $331 \mathrm{mg}, 93 \%$ yield, m.p. $=90-91{ }^{\circ} \mathrm{C} .{ }^{1} \mathrm{H}$ NMR $\left(400 \mathrm{MHz}, \mathrm{CDCl}_{3}\right) \delta$ 8.27 (s, 1H), 7.76-7.72 (m, 3H), $7.61(\mathrm{t}, J=8.8 \mathrm{~Hz}, 2 \mathrm{H}), 7.50(\mathrm{~s}, 1 \mathrm{H}), 7.21$ (d, $J=7.6$ $\mathrm{Hz}, 2 \mathrm{H}), 7.08$ (d, $J=8.8 \mathrm{~Hz}, 1 \mathrm{H}), 6.84$ (d, $J=2.8 \mathrm{~Hz}, 1 \mathrm{H}), 6.51(\mathrm{~s}, 1 \mathrm{H}), 6.05$ (brs, 1H), $4.20(\mathrm{~s}, 2 \mathrm{H}), 3.50-3.43(\mathrm{~m}, 4 \mathrm{H}), 2.35(\mathrm{~s}, 3 \mathrm{H}), 2.13(\mathrm{~s}, 1 \mathrm{H}) .{ }^{13} \mathrm{C} \mathrm{NMR}\left(100 \mathrm{MHz}, \mathrm{CDCl}_{3}\right)$ $\delta 154.3,152.4,143.8,142.2,135.2,133.4,129.6,129.2,128.9,128.3,127.6,119.5$, 118.0, 116.9, 115.8, 112.0, 105.9, 77.1, 74.1, 46.2, 37.6, 24.8, 21.5; IR (film): $\mathrm{V}_{\max }\left(\mathrm{cm}^{-}\right.$ $\left.{ }^{1}\right)=3405,3285,1734,1625,1598,1578,1514,1491,1443,1373,1352,1325,1302$, 1284, 1245, 1218, 1160, 1130, 1096, 1067, 1046, 1007, 951, 927, 897, 832, 812, 780, 759, 737, 718, 685, 661, 577, 548, 510, 492, 439; HRMS (ESI): Exact mass calcd. for $\mathrm{C}_{26} \mathrm{H}_{24} \mathrm{NO}_{4} \mathrm{~S}\left([\mathrm{M}+\mathrm{H}]^{+}\right): 446.1421$. Found: 446.1431 . 


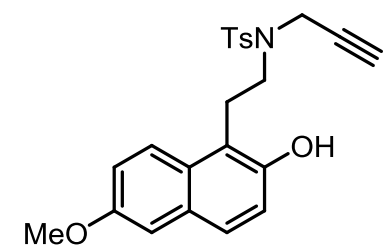

1n: pale white solid, $345 \mathrm{mg}, 88 \%$ yield, m.p. $=69-71{ }^{\circ} \mathrm{C} .{ }^{1} \mathrm{H} \mathrm{NMR}\left(400 \mathrm{MHz}, \mathrm{CDCl}_{3}\right)$ $\delta 7.83(\mathrm{~d}, J=9.6 \mathrm{~Hz}, 1 \mathrm{H}), 7.71(\mathrm{~d}, J=8.4 \mathrm{~Hz}, 2 \mathrm{H}), 7.53(\mathrm{~d}, J=8.8 \mathrm{~Hz}, 1 \mathrm{H}), 7.22(\mathrm{~d}, J$ $=8.0 \mathrm{~Hz}, 2 \mathrm{H}), 7.17-7.14(\mathrm{~m}, 1 \mathrm{H}), 7.09-7.07(\mathrm{~m}, 2 \mathrm{H}), 5.88$ (brs, $1 \mathrm{H}), 4.16(\mathrm{~d}, J=2.0$ $\mathrm{Hz}, 2 \mathrm{H}), 3.89$ (s, 3H), 3.40 (s, 4H), 2.37 (s, 3H), 2.10 (s, 1H). ${ }^{13} \mathrm{C}$ NMR (100 MHz, $\left.\mathrm{CDCl}_{3}\right) \delta 155.6,150.2,143.7,135.3,130.1,129.6,128.4,127.7,127.2,124.0,119.2$, $118.5,115.9,106.9,77.2,73.9,55.3,46.3,37.6,25.0,21.5$; IR (film): $V_{\max }\left(\mathrm{cm}^{-1}\right)=$ $3413,3300,2931,1611,1591,1513,1460,1440,1377,1348,1324,1283,1249,1157$, 1093, 1064, 1025, 940, 892, 855, 808, 775, 751, 720, 661, 628, 579, 545, 531, 505, 429; HRMS (ESI): Exact mass calcd. for $\mathrm{C}_{23} \mathrm{H}_{24} \mathrm{NO}_{4} \mathrm{~S}\left([\mathrm{M}+\mathrm{H}]^{+}\right)$: 410.1421. Found: 410.1430 .

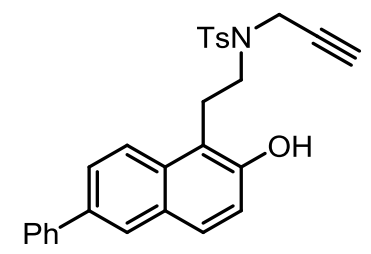

10: pale white solid, $1.51 \mathrm{~g}, 93 \%$ yield, m.p. $=70-71{ }^{\circ} \mathrm{C} .{ }^{1} \mathrm{H} \mathrm{NMR}\left(400 \mathrm{MHz}, \mathrm{CDCl}_{3}\right)$ $\delta 7.99(\mathrm{~d}, J=8.8 \mathrm{~Hz}, 1 \mathrm{H}), 7.96(\mathrm{~d}, J=1.6 \mathrm{~Hz}, 1 \mathrm{H}), 7.77-7.68(\mathrm{~m}, 6 \mathrm{H}), 7.47(\mathrm{t}, J=8.0$ $\mathrm{Hz}, 2 \mathrm{H}), 7.35$ (t, $J=7.6 \mathrm{~Hz}, 1 \mathrm{H}), 7.24$ (d, $J=8.8 \mathrm{~Hz}, 2 \mathrm{H}), 7.14(\mathrm{~d}, J=8.8 \mathrm{~Hz}, 1 \mathrm{H})$, 5.98 (brs, 1H), 4.18 (d, $J=2.4 \mathrm{~Hz}, 2 \mathrm{H}), 3.45$ (s, 4H), 2.37 (s, 3H), 2.11 (t, $J=2.4 \mathrm{~Hz}$, 1H). ${ }^{13} \mathrm{C} \mathrm{NMR}\left(100 \mathrm{MHz}, \mathrm{CDCl}_{3}\right) \delta 152.0,143.8,140.8,135.8,135.3,132.3,129.6$, $129.5,128.9,128.8,127.7,127.1,126.5,126.3,123.0,118.5,115.4,77.2,73.8,46.4$, 37.8, 25.2, 21.5; IR (film): $V_{\max }\left(\mathrm{cm}^{-1}\right)=3397,3294,1627,1597,1574,1494,1445$, 1363, 1321, 1288, 1187, 1148, 1096, 1034, 1017, 1002, 923, 905, 891, 807, 757, 743, 721, 698, 658, 580, 542, 482, 425; HRMS (ESI): Exact mass calcd. for $\mathrm{C}_{28} \mathrm{H}_{26} \mathrm{NO}_{3} \mathrm{~S}$ $\left([\mathrm{M}+\mathrm{H}]^{+}\right): 456.1628$. Found: 456.1635 . 


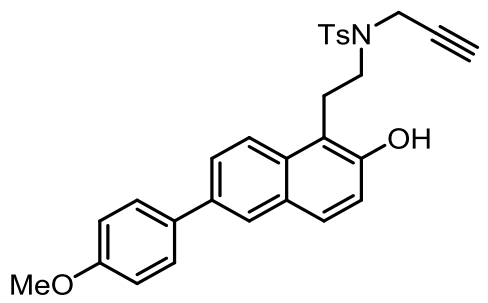

1p: pale white solid, $835 \mathrm{mg}, 86 \%$ yield, m.p. $=135-137{ }^{\circ} \mathrm{C} .{ }^{1} \mathrm{H} \mathrm{NMR}\left(400 \mathrm{MHz}, \mathrm{CDCl}_{3}\right)$ $\delta 7.97(\mathrm{~d}, J=8.8 \mathrm{~Hz}, 1 \mathrm{H}), 7.91(\mathrm{~s}, 1 \mathrm{H}), 7.73-7.71(\mathrm{~m}, 3 \mathrm{H}), 7.68(\mathrm{~d}, J=9.2 \mathrm{~Hz}, 1 \mathrm{H})$, $7.62(\mathrm{~d}, J=8.4 \mathrm{~Hz}, 2 \mathrm{H}), 7.25-7.22(\mathrm{~m}, 2 \mathrm{H}), 7.12(\mathrm{~d}, J=8.8 \mathrm{~Hz}, 1 \mathrm{H}), 7.01(\mathrm{~d}, J=8.8$ Hz, 2H), 5.97 (brs, 1H), 4.18 (d, $J=1.6 \mathrm{~Hz}, 2 \mathrm{H}), 3.86$ (s, 3H), 3.44 (s, 4H), 2.37 (s, 3H), $2.11(\mathrm{~s}, 1 \mathrm{H}) .{ }^{13} \mathrm{C} \mathrm{NMR}\left(100 \mathrm{MHz}, \mathrm{CDCl}_{3}\right) \delta 159.0,151.7,143.7,135.4,135.3$, $133.4,132.0,129.6,128.8,128.1,127.7,126.2,125.7,122.9,118.5,115.3,114.3,77.2$, 73.8, 55.4, 46.4, 37.8, 25.1, 21.5; IR (film): $\mathrm{V}_{\max }\left(\mathrm{cm}^{-1}\right)=3480,3265,1630,1606,1581$, $1518,1500,1460,1416,1361,1343,1314,1281,1247,1159,1127,1096,945,928$, 913, 890, 842, 809, 763, 749, 699, 676, 658, 581, 546, 478, 441, 426; HRMS (ESI): Exact mass calcd. for $\mathrm{C}_{29} \mathrm{H}_{28} \mathrm{NO}_{4} \mathrm{~S}\left([\mathrm{M}+\mathrm{H}]^{+}\right)$: 486.1734. Found: 486.1733.

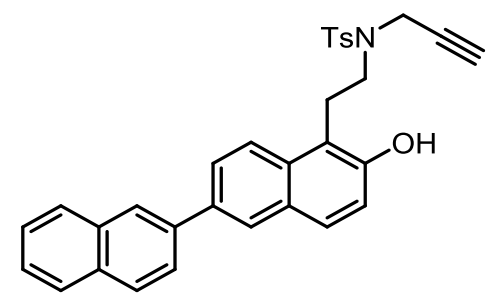

1q: pale white solid, $620 \mathrm{mg}, 75 \%$ yield, m.p. $=163-165{ }^{\circ} \mathrm{C} .{ }^{1} \mathrm{H}$ NMR $(400 \mathrm{MHz}$, $\left.\left(\mathrm{CD}_{3}\right)_{2} \mathrm{CO}\right) \delta 8.84(\mathrm{brs}, 1 \mathrm{H}), 8.31(\mathrm{~s}, 1 \mathrm{H}), 8.24(\mathrm{~s}, 1 \mathrm{H}), 8.17(\mathrm{~d}, J=8.8 \mathrm{~Hz}, 1 \mathrm{H}), 8.02-$ 7.97 (m, 3H), 7.94 (d, $J=7.6 \mathrm{~Hz}, 1 \mathrm{H}), 7.82$ (d, $J=10.0 \mathrm{~Hz}, 1 \mathrm{H}), 7.74$ (d, $J=7.2 \mathrm{~Hz}$, 2H), 7.56-7.49 (m, 2H), $7.32(\mathrm{~d}, J=7.6 \mathrm{~Hz}, 2 \mathrm{H}), 7.28$ (d, J=8.8 Hz, 1H), 4.39 (s, 2H), 3.50-3.48 (m, 4H), $3.01(\mathrm{~s}, 1 \mathrm{H}), 2.75(\mathrm{~s}, 1 \mathrm{H}), 2.35$ (s, 3H). ${ }^{13} \mathrm{C}$ NMR (100 MHz, $\left.\left(\mathrm{CD}_{3}\right)_{2} \mathrm{CO}\right) \delta 153.0,143.4,138.1,136.5,134.7,134.0,133.0,132.7,129.5,129.4,128.7$ $128.5,128.2,127.6,127.5,126.6,126.3,125.9,125.3,123.4,118.2,115.8,77.5,74.5$, 45.9, 36.6, 24.0, 20.5; IR (film): $\mathrm{v}_{\max }\left(\mathrm{cm}^{-1}\right)=3483,3290,1626,1597,1577,1505$, 1484, 1463, 1444, 1426, 1362, 1347, 1310, 1278, 1199, 1164, 1120, 1094, 1017, 952, 916, 900, 885, 861, 836, 808, 747, 713, 682, 644, 579, 546, 473, 437; HRMS (ESI): Exact mass calcd. for $\mathrm{C}_{32} \mathrm{H}_{28} \mathrm{NO}_{3} \mathrm{~S}\left([\mathrm{M}+\mathrm{H}]^{+}\right)$: 506.1784. Found: 506.1784. 


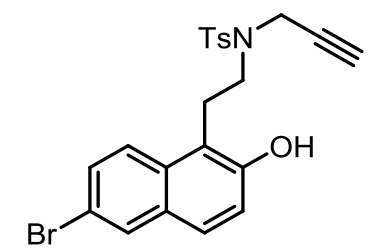

1r: brown solid, $236 \mathrm{mg}, 92 \%$ yield, m.p. $=56-58{ }^{\circ} \mathrm{C} .{ }^{1} \mathrm{H}$ NMR $\left(400 \mathrm{MHz}, \mathrm{CDCl}_{3}\right) \delta$ $7.89(\mathrm{~d}, J=1.2 \mathrm{~Hz}, 1 \mathrm{H}), 7.78(\mathrm{~d}, J=9.2 \mathrm{~Hz}, 1 \mathrm{H}), 7.70(\mathrm{~d}, J=8.0 \mathrm{~Hz}, 2 \mathrm{H}), 7.56-7.51$ (m, 2H), 7.24 (d, $J=8.0 \mathrm{~Hz}, 2 \mathrm{H}), 7.13$ (d, $J=8.8 \mathrm{~Hz}, 1 \mathrm{H}), 6.12$ (brs, 1H), 4.15 (d, $J=$ $2.4 \mathrm{~Hz}, 2 \mathrm{H}), 3.39$ (s, 4H), 2.39 (s, 3H), 2.10 (s, 1H). ${ }^{13} \mathrm{C} \mathrm{NMR}\left(100 \mathrm{MHz}, \mathrm{CDCl}_{3}\right) \delta$ 152.2, 143.9, 135.0, 131.7, 130.5, 130.3, 129.9, 129.6, 127.7, 127.6, 124.2, 119.2, 116.8, 115.7, 77.1, 73.8, 46.2, 37.8, 25.1, 21.6; IR (film): $\mathrm{v}_{\max }\left(\mathrm{cm}^{-1}\right)=3428,3291,1657,1624$, 1594, 1499, 1454, 1419, 1325, 1305, 1267, 1193, 1150, 1094, 1016, 1003, 901, 880, 809, 738, 706, 656, 571, 544, 493, 423; HRMS (ESI): Exact mass calcd. for $\mathrm{C}_{22} \mathrm{H}_{21} \mathrm{BrNO}_{3} \mathrm{~S}\left([\mathrm{M}+\mathrm{H}]^{+}\right)$: 458.0420. Found: 458.0417 .

\subsection{General procedure for gold-catalyzed intramolecular dearomatization} reaction of $\beta$-naphthol derivatives<smiles>C#CCN(CCCc1c(O)ccc2ccccc12)C(=O)O</smiles>

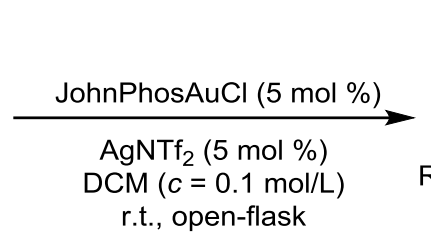

r.t., open-flask

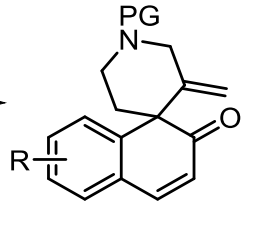

2

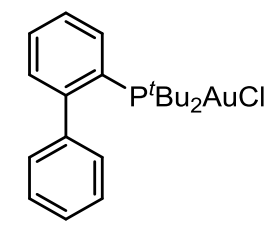

JohnPhosAuCl

To a dried test tube were added 1 (0.2 mmol), JohnPhosAuCl (5.3 mg, $0.01 \mathrm{mmol}$, $5 \mathrm{~mol} \%)$ and $\operatorname{DCM}(2.0 \mathrm{~mL})$, then $\operatorname{AgNTf}_{2}(3.9 \mathrm{mg}, 0.01 \mathrm{mmol}, 5 \mathrm{~mol} \%)$. The reaction mixture was stirred at room temperature. After the reaction was complete (monitored by TLC or LC-MS), it was quenched by brine $(0.1 \mathrm{~mL})$. The mixture was then filtered through celite and washed by EtOAc $(30 \mathrm{~mL})$. The mixture was concentrated in vacuo. Then the residue was purified by silica gel column chromatography (PE/acetone $=10: 1)$ to afford the desired product (2a-2r). The analytical data of the products are summarized below. 


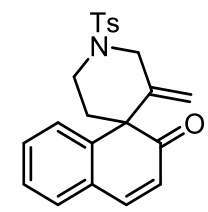

2a: white solid, $75.8 \mathrm{mg}$, 94\% yield, m.p. $=151-153{ }^{\circ} \mathrm{C} .{ }^{1} \mathrm{H}$ NMR $\left(400 \mathrm{MHz}, \mathrm{CDCl}_{3}\right)$ $\delta 7.73(\mathrm{~d}, J=7.6 \mathrm{~Hz}, 2 \mathrm{H}), 7.37-7.29(\mathrm{~m}, 6 \mathrm{H}), 7.16(\mathrm{~d}, J=7.2 \mathrm{~Hz}, 1 \mathrm{H}), 5.90(\mathrm{~d}, J=9.6$ $\mathrm{Hz}, 1 \mathrm{H}), 5.08(\mathrm{~s}, 1 \mathrm{H}), 4.33(\mathrm{~s}, 1 \mathrm{H}), 4.33$ (d, $J=12.8 \mathrm{~Hz}, 1 \mathrm{H}), 3.82-3.79(\mathrm{~m}, 2 \mathrm{H}), 3.30$ (t, $J=12.0 \mathrm{~Hz}, 1 \mathrm{H}), 2.47(\mathrm{~s}, 3 \mathrm{H}), 2.26$ (td, $J=13.6,4.8 \mathrm{~Hz}, 1 \mathrm{H}), 1.89$ (d, $J=14.0 \mathrm{~Hz}$, 1H). ${ }^{13} \mathrm{C}$ NMR (100 MHz, $\left.\mathrm{CDCl}_{3}\right) \delta 200.9,145.2,143.8,143.6,141.7,134.0,130.2$, $130.1,129.81,129.77,129.4,127.8,127.5,123.2,116.8,54.2,50.6,42.2,36.0,21.6$; IR (film): $\mathrm{V}_{\max }\left(\mathrm{cm}^{-1}\right)=2968,2946,2921,2883,2850,1649,1597,1564,1492,1461$, $1436,1397,1339,1329,1311,1286,1252,1236,1217,1155,1135,1097,1031,1002$, 960, 915, 872, 840, 816, 751, 707, 686, 658, 602, 545, 528, 510, 467, 452; HRMS (ESI): Exact mass calcd. for $\mathrm{C}_{22} \mathrm{H}_{22} \mathrm{NO}_{3} \mathrm{~S}\left([\mathrm{M}+\mathrm{H}]^{+}\right)$: 380.1315 . Found: 380.1326 .

\section{The procedure for a gram-scale reaction of product 2a:}

To a dried tube were added $1 \mathrm{a}(1.14 \mathrm{~g}, 3.0 \mathrm{mmol})$, JohnPhosAuCl (79.5 mg, 0.15 mmol, $5 \mathrm{~mol} \%)$ and DCM (30.0 mL), then $\operatorname{AgNTf}_{2}(58.5 \mathrm{mg}, 0.15 \mathrm{mmol}, 5 \mathrm{~mol} \%)$. The reaction mixture was stirred at room temperature. After the reaction was complete (monitored by TLC or LC-MS), it was quenched by brine $(1.5 \mathrm{~mL})$. The mixture was then filtered through celite and washed by EtOAc $(60 \mathrm{~mL})$. The mixture was concentrated in vacuo. Then the residue was purified by silica gel column chromatography $(\mathrm{PE} /$ acetone $=10: 1)$ to afford the desired product 2a $(1.06 \mathrm{~g}, 93 \%$ yield).

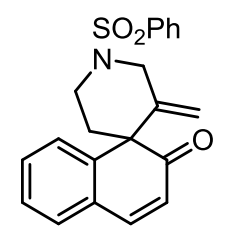

2b: white solid, $72.5 \mathrm{mg}$, 99\% yield, m.p. $=167-168{ }^{\circ} \mathrm{C} .{ }^{1} \mathrm{H}$ NMR $\left(400 \mathrm{MHz}, \mathrm{CDCl}_{3}\right)$ $\delta 7.86(\mathrm{~d}, J=7.6 \mathrm{~Hz}, 2 \mathrm{H}), 7.65(\mathrm{t}, J=7.2 \mathrm{~Hz}, 1 \mathrm{H}), 7.60-7.56(\mathrm{~m}, 2 \mathrm{H}), 7.38-7.29(\mathrm{~m}$, 4H), $7.17(\mathrm{~d}, J=7.6 \mathrm{~Hz}, 1 \mathrm{H}), 5.90(\mathrm{~d}, J=9.6 \mathrm{~Hz}, 1 \mathrm{H}), 5.10(\mathrm{~s}, 1 \mathrm{H}), 4.35(\mathrm{~d}, J=13.2$ $\mathrm{Hz}, 1 \mathrm{H}), 4.34(\mathrm{~s}, 1 \mathrm{H}), 3.84-3.81(\mathrm{~m}, 2 \mathrm{H}), 3.33(\mathrm{td}, J=12.4,2.8 \mathrm{~Hz}, 1 \mathrm{H}), 2.27(\mathrm{td}, J=$ 
13.2, 4.8 Hz, 1H), $1.90(\mathrm{~d}, J=14.4 \mathrm{~Hz}, 1 \mathrm{H}) .{ }^{13} \mathrm{C} \mathrm{NMR}\left(100 \mathrm{MHz}, \mathrm{CDCl}_{3}\right) \delta 200.9$, 145.2, 143.8, 141.6, 137.1, 132.8, 130.3, 130.1, 129.7, 129.4, 129.2, 127.7, 127.6, 123.2, 116.9, 54.2, 50.5, 42.2, 36.0; IR (film): $\mathrm{v}_{\max }\left(\mathrm{cm}^{-1}\right)=2925,2884,2851,1647,1595$, 1564, 1460, 1446, 1396, 1370, 1344, 1328, 1311, 1284, 1250, 1235, 1216, 1200, 1155, 1134, 1098, 1071, 1029, 1001, 959, 930, 913, 872, 840, 811, 769, 750, 718, 689, 657, 621, 604, 573, 552, 526, 508, 448; HRMS (ESI): Exact mass calcd. for $\mathrm{C}_{21} \mathrm{H}_{20} \mathrm{NO}_{3} \mathrm{~S}$ $\left([\mathrm{M}+\mathrm{H}]^{+}\right): 366.1158$. Found: 366.1169 .

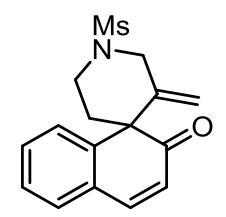

2c: pale yellow solid, $57.5 \mathrm{mg}, 95 \%$ yield, m.p. $=73-75^{\circ} \mathrm{C} .{ }^{1} \mathrm{H} \mathrm{NMR}\left(400 \mathrm{MHz}, \mathrm{CDCl}_{3}\right)$ 反 7.45-7.44 (m, 3H), 7.354-7.348 (m, 2H), $6.01(\mathrm{~d}, J=10.0 \mathrm{~Hz}, 1 \mathrm{H}), 5.12(\mathrm{~s}, 1 \mathrm{H}), 4.41$ $(\mathrm{s}, 1 \mathrm{H}), 4.28\left(\mathrm{AB}, J_{A B}=13.6 \mathrm{~Hz}, 1 \mathrm{H}\right), 4.12\left(\mathrm{BA}, J_{B A}=13.2 \mathrm{~Hz}, 1 \mathrm{H}\right), 3.76(\mathrm{~d}, J=9.6$ $\mathrm{Hz}, 1 \mathrm{H}), 3.62(\mathrm{td}, J=12.0,2.4 \mathrm{~Hz}, 1 \mathrm{H}), 2.92(\mathrm{~s}, 3 \mathrm{H}), 2.36(\mathrm{td}, J=14.0,4.8 \mathrm{~Hz}, 1 \mathrm{H})$, $2 . .0(\mathrm{~d}, J=14.4 \mathrm{~Hz}, 1 \mathrm{H}) .{ }^{13} \mathrm{C} \mathrm{NMR}\left(100 \mathrm{MHz}, \mathrm{CDCl}_{3}\right) \delta 201.0,145.4,143.8,141.7$, 130.4, 130.1, 129.7, 129.5, 127.7, 123.3, 116.9, 54.3, 50.1, 41.8, 36.3, 35.1; IR (film): $\mathrm{V}_{\max }\left(\mathrm{cm}^{-1}\right)=2927,2879,1648,1597,1565,1487,1457,1435,1399,1323,1282,1249$, 1227, 1150, 1096, 1031, 1004, 958, 914, 877, 832, 812, 774, 752, 704, 658, 609, 585, 521, 504, 458, 439; HRMS (ESI): Exact mass calcd. for $\mathrm{C}_{16} \mathrm{H}_{18} \mathrm{NO}_{3} \mathrm{~S}\left([\mathrm{M}+\mathrm{H}]^{+}\right)$: 304.1002. Found: 304.1011.

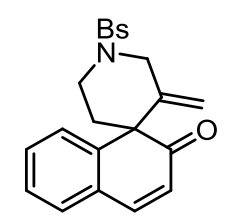

2d: white solid, $86.3 \mathrm{mg}$, 97\% yield, m.p. $=152-153{ }^{\circ} \mathrm{C} .{ }^{1} \mathrm{H} \mathrm{NMR}\left(400 \mathrm{MHz}, \mathrm{CDCl}_{3}\right)$ $\delta 7.72(\mathrm{~s}, 3 \mathrm{H}), 7.42-7.37(\mathrm{~m}, 2 \mathrm{H}), 7.34-7.30(\mathrm{~m}, 2 \mathrm{H}), 7.26(\mathrm{~s}, 1 \mathrm{H}), 7.11(\mathrm{~d}, J=8.0 \mathrm{~Hz}$, $1 \mathrm{H}), 5.92(\mathrm{~d}, J=10.0 \mathrm{~Hz}, 1 \mathrm{H}), 5.10(\mathrm{~s}, 1 \mathrm{H}), 4.35(\mathrm{~s}, 1 \mathrm{H}), 4.34\left(\mathrm{AB}, J_{A B}=14.4 \mathrm{~Hz}, 1 \mathrm{H}\right)$, $3.86\left(\mathrm{BA}, J_{B A}=14.0 \mathrm{~Hz}, 1 \mathrm{H}\right), 3.83-3.79(\mathrm{~m}, 1 \mathrm{H}), 3.36(\mathrm{td}, J=12.4,3.2 \mathrm{~Hz}, 1 \mathrm{H}), 2.25$ $(\mathrm{td}, J=14.0,2.8 \mathrm{~Hz}, 1 \mathrm{H}), 1.90(\mathrm{~d}, J=14.0 \mathrm{~Hz}, 1 \mathrm{H}) .{ }^{13} \mathrm{C} \mathrm{NMR}\left(100 \mathrm{MHz}, \mathrm{CDCl}_{3}\right) \delta$ 200.8, 145.3, 143.6, 141.3, 136.4, 132.5, 130.4, 130.1, 129.6, 129.5, 129.3, 127.9, 127.7, 123.2, 117.1, 54.1, 50.5, 42.2, 35.8; IR (film): $\mathrm{v}_{\max }\left(\mathrm{cm}^{-1}\right)=3080,3022,2923,2852$, 
$1647,1596,1572,1489,1461,1435,1391,1343,1314,1284,1251,1235,1218,1155$, 1134, 1098, 1067, 1031, 1003, 957, 934, 915, 874, 839, 824, 811, 766, 749, 705, 683, 657, 622, 607, 587, 552, 527, 508, 486, 465, 451, 421; HRMS (ESI): Exact mass calcd. for $\mathrm{C}_{21} \mathrm{H}_{19} \mathrm{BrNO}_{3} \mathrm{~S}\left([\mathrm{M}+\mathrm{H}]^{+}\right)$: 444.0264. Found: 444.0266 .

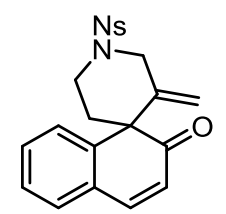

2e: white solid, $82.6 \mathrm{mg}$, 99\% yield, m.p. $=154-155^{\circ} \mathrm{C} .{ }^{1} \mathrm{H} \mathrm{NMR}\left(400 \mathrm{MHz}, \mathrm{CDCl}_{3}\right)$ $\delta 8.43(\mathrm{~d}, J=8.4 \mathrm{~Hz}, 2 \mathrm{H}), 8.04(\mathrm{~d}, J=8.4 \mathrm{~Hz}, 2 \mathrm{H}), 7.41-7.32(\mathrm{~m}, 4 \mathrm{H}), 7.22(\mathrm{~d}, J=7.6$ $\mathrm{Hz}, 1 \mathrm{H}), 5.91(\mathrm{~d}, J=9.6 \mathrm{~Hz}, 1 \mathrm{H}), 5.13(\mathrm{~s}, 1 \mathrm{H}), 4.39(\mathrm{~s}, 1 \mathrm{H}), 4.37\left(\mathrm{AB}, J_{A B}=14.8 \mathrm{~Hz}\right.$, $1 \mathrm{H}), 3.87\left(\mathrm{BA}, J_{B A}=13.2 \mathrm{~Hz}, 1 \mathrm{H}\right), 3.85-3.82(\mathrm{~m}, 1 \mathrm{H}), 3.39$ (td, $\left.J=11.6,1.6 \mathrm{~Hz}, 1 \mathrm{H}\right)$, $2.30(\mathrm{td}, J=14.0,5.2 \mathrm{~Hz}, 1 \mathrm{H}), 1.94(\mathrm{~d}, J=14.4 \mathrm{~Hz}, 1 \mathrm{H}) .{ }^{13} \mathrm{C} \mathrm{NMR}\left(100 \mathrm{MHz}, \mathrm{CDCl}_{3}\right)$ $\delta 200.7,150.2,145.4,143.3,143.0,141.0,130.4,130.1,129.6,129.5,128.9,127.8$, 124.5, 123.1, 117.3, 54.0, 50.4, 42.2, 36.0; IR (film): $\mathrm{v}_{\max }\left(\mathrm{cm}^{-1}\right)=3109,2922,2890$, 2852, 1646, 1597, 1565, 1532, 1462, 1436, 1397, 1366, 1344, 1309, 1285, 1251, 1235, $1217,1159,1132,1098,1030,1001,956,934,913,871,853,838,811,766,748,698$, 681, 657, 623, 608, 591, 549, 521, 453; HRMS (ESI): Exact mass calcd. for $\mathrm{C}_{21} \mathrm{H}_{19} \mathrm{~N}_{2} \mathrm{O}_{5} \mathrm{~S}\left([\mathrm{M}+\mathrm{H}]^{+}\right)$: 411.1009. Found: 41.1011.

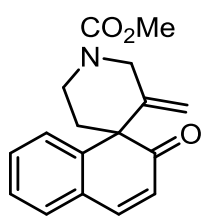

2f: white solid, $43.1 \mathrm{mg}, 76 \%$ yield, m.p. $=69-70{ }^{\circ} \mathrm{C} .{ }^{1} \mathrm{H}$ NMR $\left(400 \mathrm{MHz}, \mathrm{CDCl}_{3}\right) \delta$ 7.43-7.38 (m, 2H), 7.36-7.28 (m, 3H), $6.03(\mathrm{~d}, J=10.0 \mathrm{~Hz}, 1 \mathrm{H}), 5.09$ (d, $J=15.2 \mathrm{~Hz}$, $1 \mathrm{H}), 4.65-4.46(\mathrm{~m}, 1 \mathrm{H}), 4.36(\mathrm{~s}, 1 \mathrm{H}), 4.23-4.15(\mathrm{~m}, 1 \mathrm{H}), 4.00-3.88(\mathrm{~m}, 1 \mathrm{H}), 3.76(\mathrm{~s}$, 3H), 3.72-3.70 (m, 1H), 2.10-2.06 (m, 2H). $\left.{ }^{13} \mathrm{C} \mathrm{NMR} \mathrm{(100} \mathrm{MHz,} \mathrm{CDCl}_{3}\right) \delta$ 201.2, 156.0, 144.9, 144.6, 143.0, 130.1, 130.0, 129.44, 129.40, 127.4, 123.4, 115.8, 55.4, 52.7, 48.4, 39.8, 36.3; IR (film): $v_{\max }\left(\mathrm{cm}^{-1}\right)=3080,3028,2998,2963,2924,2850,1695,1642$, $1595,1566,1474,1451,1415,1369,1349,1306,1282,1237,1194,1179,1137,1107$, $1060,1007,991,950,914,884,866,832,778,765,701,667,614,583,533,521,483$, 456, 423; HRMS (ESI): Exact mass calcd. for $\mathrm{C}_{17} \mathrm{H}_{18} \mathrm{NO}_{3}\left([\mathrm{M}+\mathrm{H}]^{+}\right)$: 284.1281. Found: 
284.1292 .

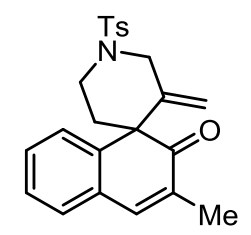

2g: white solid, $78.1 \mathrm{mg}, 92 \%$ yield, m.p. $=180-181{ }^{\circ} \mathrm{C} .{ }^{1} \mathrm{H} \mathrm{NMR}\left(400 \mathrm{MHz}, \mathrm{CDCl}_{3}\right)$ $\delta 7.73(\mathrm{~d}, J=8.0 \mathrm{~Hz}, 2 \mathrm{H}), 7.36(\mathrm{~d}, J=8.0 \mathrm{~Hz}, 2 \mathrm{H}), 7.30-7.22(\mathrm{~m}, 4 \mathrm{H}), 7.11-7.09$ (m, $1 \mathrm{H}), 5.06(\mathrm{~s}, 1 \mathrm{H}), 4.34\left(\mathrm{AB}, J_{A B}=13.2 \mathrm{~Hz}, 1 \mathrm{H}\right), 4.32(\mathrm{~s}, 1 \mathrm{H}), 3.82\left(\mathrm{BA}, J_{B A}=13.6 \mathrm{~Hz}\right.$, $1 \mathrm{H}), 3.83-3.80(\mathrm{~m}, 1 \mathrm{H}), 3.32(\mathrm{td}, J=12.0,2.4 \mathrm{~Hz}, 1 \mathrm{H}), 2.47(\mathrm{~s}, 3 \mathrm{H}), 2.25(\mathrm{td}, J=13.2$, $4.8 \mathrm{~Hz}, 1 \mathrm{H}), 1.87-1.84(\mathrm{~m}, 4 \mathrm{H}) .{ }^{13} \mathrm{C} \mathrm{NMR}\left(100 \mathrm{MHz}, \mathrm{CDCl}_{3}\right) \delta 201.1,143.6,143.4$, 141.99, 141.97, 134.2, 130.6, 130.1, 129.8, 129.5, 129.1, 128.5, 127.8, 127.4, 116.7, 53.8, 50.6, 42.2, 36.2, 21.6, 15.9; IR (film): $\mathrm{V}_{\max }\left(\mathrm{cm}^{-1}\right)=2966,2954,2926,2893,2849$, 1644, 1596, 1572, 1491, 1458, 1441, 1373, 1348, 1309, 1281, 1256, 1217, 1195, 1161, 1136, 1089, 1057, 1019, 999, 980, 950, 906, 871, 858, 817, 775, 759, 746, 701, 656, 603, 557, 544, 522, 478, 460; HRMS (ESI): Exact mass calcd. for $\mathrm{C}_{23} \mathrm{H}_{24} \mathrm{NO}_{3} \mathrm{~S}$ $\left([\mathrm{M}+\mathrm{H}]^{+}\right): 394.1471$. Found: 394.1483.

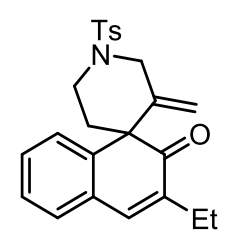

2h: white solid, $73.3 \mathrm{mg}, 90 \%$ yield, m.p. $=182-183{ }^{\circ} \mathrm{C} .{ }^{1} \mathrm{H} \mathrm{NMR}\left(400 \mathrm{MHz}, \mathrm{CDCl}_{3}\right)$ $\delta 7.73(\mathrm{~d}, J=8.0 \mathrm{~Hz}, 2 \mathrm{H}), 7.36(\mathrm{~d}, J=8.0 \mathrm{~Hz}, 2 \mathrm{H}), 7.28-7.26(\mathrm{~m}, 3 \mathrm{H}), 7.15-7.11(\mathrm{~m}$, 2H), $5.07(\mathrm{~s}, 1 \mathrm{H}), 4.34\left(\mathrm{AB}, J_{A B}=12.8 \mathrm{~Hz}, 1 \mathrm{H}\right), 4.33(\mathrm{~s}, 1 \mathrm{H}), 3.81-3.78(\mathrm{~m}, 1 \mathrm{H}), 3.80$ $\left(\mathrm{BA}, J_{B A}=13.3 \mathrm{~Hz}, 1 \mathrm{H}\right), 3.31(\mathrm{td}, J=12.4,2.8 \mathrm{~Hz}, 1 \mathrm{H}), 2.47(\mathrm{~s}, 3 \mathrm{H}), 2.31-2.21(\mathrm{~m}$, $3 \mathrm{H}), 1.87(\mathrm{~d}, J=14.0 \mathrm{~Hz}, 1 \mathrm{H}), 1.05(\mathrm{t}, J=7.2 \mathrm{~Hz}, 3 \mathrm{H}) .{ }^{13} \mathrm{C} \mathrm{NMR}\left(100 \mathrm{MHz}, \mathrm{CDCl}_{3}\right)$ $\delta 200.8,143.6,143.2,141.9,140.0,135.6,134.3,130.7,129.8,129.3,129.1,128.7$, $127.7,127.4,116.6,54.0,50.6,42.3,36.0,22.2,21.6,12.5$; IR (film): $v_{\max }\left(\mathrm{cm}^{-1}\right)=$ 2965, 2929, 2870, 2852, 1652, 1633, 1595, 1572, 1492, 1459, 1380, 1343, 1330, 1307, 1280, 1258, 1238, 1218, 1190, 1166, 1156, 1134, 1107, 1090, 1037, 1012, 1000, 955, 921, 901, 871, 814, 791, 759, 706, 659, 604, 560, 544, 524, 498, 485, 473, 458; HRMS (ESI): Exact mass calcd. for $\mathrm{C}_{24} \mathrm{H}_{26} \mathrm{NO}_{3} \mathrm{~S}\left([\mathrm{M}+\mathrm{H}]^{+}\right)$: 408.1628 . Found: 408.1642 . 


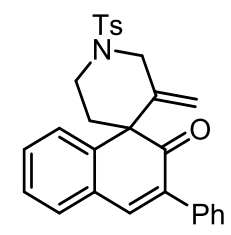

2i: white solid, $81.4 \mathrm{mg}, 89 \%$ yield, m.p. $=171-173{ }^{\circ} \mathrm{C} .{ }^{1} \mathrm{H}$ NMR $\left(400 \mathrm{MHz}, \mathrm{CDCl}_{3}\right) \delta$ $7.72(\mathrm{~d}, J=7.6 \mathrm{~Hz}, 2 \mathrm{H}), 7.44$ (s, 1H), 7.39-7.33 (m, 9H), 7.26 (s, 1H), 7.23 (d, $J=7.6$ $\mathrm{Hz}, 1 \mathrm{H}), 5.14(\mathrm{~s}, 1 \mathrm{H}), 4.44(\mathrm{~s}, 1 \mathrm{H}), 4.36\left(\mathrm{AB}, J_{A B}=13.6 \mathrm{~Hz}, 1 \mathrm{H}\right), 3.83(\mathrm{dd}, J=13.2$, $3.6 \mathrm{~Hz}, 1 \mathrm{H}), 3.77\left(\mathrm{BA}, J_{B A}=13.2 \mathrm{~Hz}, 1 \mathrm{H}\right), 3.33(\mathrm{t}, J=12.4 \mathrm{~Hz}, 1 \mathrm{H}), 2.46(\mathrm{~s}, 3 \mathrm{H}), 2.36$ $(\operatorname{td}, J=13.6,5.2 \mathrm{~Hz}, 1 \mathrm{H}), 2.05(\mathrm{~d}, J=14.0 \mathrm{~Hz}, 1 \mathrm{H}) .{ }^{13} \mathrm{C} \mathrm{NMR}\left(100 \mathrm{MHz}, \mathrm{CDCl}_{3}\right) \delta$ 199.6, 143.7, 143.6, 142.5, 141.7, 135.5, 134.1, 133.4, 130.6, 130.0, 129.9, 129.6, 129.4, 128.6, 128.24, 128.17, 127.8, 127.7, 117.2, 54.9, 50.6, 42.6, 36.0, 21.6; IR (film): $v_{\max }$ $\left(\mathrm{cm}^{-1}\right)=2963,2926,2881,1649,1596,1572,1491,1460,1429,1397,1363,1340$, $1286,1222,1186,1158,1095,1025,1003,959,921,898,845,817,801,788,765,753$, 741, 702, 658, 601, 569, 550, 538, 517, 490, 461, 414; HRMS (ESI): Exact mass calcd. for $\mathrm{C}_{28} \mathrm{H}_{26} \mathrm{NO}_{3} \mathrm{~S}\left([\mathrm{M}+\mathrm{H}]^{+}\right)$: 456.1628. Found: 456.1636 .

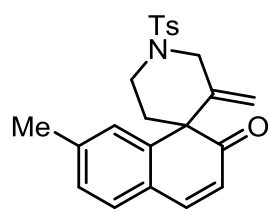

2j: white solid, $74.8 \mathrm{mg}, 95 \%$ yield, m.p. $=125-126{ }^{\circ} \mathrm{C} .{ }^{1} \mathrm{H}$ NMR $\left(400 \mathrm{MHz}, \mathrm{CDCl}_{3}\right) \delta$ $7.73(\mathrm{~d}, J=8.0 \mathrm{~Hz}, 2 \mathrm{H}), 7.37-7.33(\mathrm{~m}, 3 \mathrm{H}), 7.19(\mathrm{~d}, J=7.6 \mathrm{~Hz}, 1 \mathrm{H}), 7.12-7.09(\mathrm{~m}, 2 \mathrm{H})$, $5.84(\mathrm{~d}, J=10.0 \mathrm{~Hz}, 1 \mathrm{H}), 5.08(\mathrm{~s}, 1 \mathrm{H}), 4.36(\mathrm{~s}, 1 \mathrm{H}), 4.33\left(\mathrm{AB}, J_{A B}=13.6 \mathrm{~Hz}, 1 \mathrm{H}\right)$, 3.81-3.75 (m, 2H), $3.28(\mathrm{td}, J=12 ., 2.4 \mathrm{~Hz}, 1 \mathrm{H}), 2.46(\mathrm{~s}, 3 \mathrm{H}), 2.36(\mathrm{~s}, 3 \mathrm{H}), 2.28(\mathrm{td}, J$ $=13.6,5.2 \mathrm{~Hz}, 1 \mathrm{H}), 1.89(\mathrm{~d}, J=14.0 \mathrm{~Hz}, 1 \mathrm{H}) .{ }^{13} \mathrm{C} \mathrm{NMR}\left(100 \mathrm{MHz}, \mathrm{CDCl}_{3}\right) \delta 201.1$, $145.3,143.9,143.6,141.9,140.8,133.9,130.4,129.8,129.3,128.4,127.7,127.6,122.2$, 116.7, 54.2, 50.6, 42.2, 36.2, 21.7, 21.6; IR (film): $\mathrm{V}_{\max }\left(\mathrm{cm}^{-1}\right)=2950,2925,2873,1640$, $1606,1561,1492,1458,1438,1391,1341,1328,1304,1271,1249,1223,1201,1158$, $1140,1119,1096,1021,1007,959,918,880,845,817,753,732,709,680,656,602$, 542, 494, 463; HRMS (ESI): Exact mass calcd. for $\mathrm{C}_{23} \mathrm{H}_{24} \mathrm{NO}_{3} \mathrm{~S}\left([\mathrm{M}+\mathrm{H}]^{+}\right)$: 394.1471 . Found: 394.1482. 


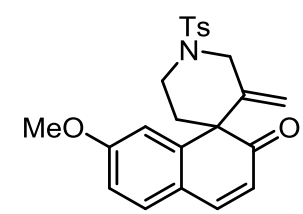

2k: white solid, $74.2 \mathrm{mg}, 91 \%$ yield, m.p. $=144-146{ }^{\circ} \mathrm{C} .{ }^{1} \mathrm{H}$ NMR $\left(400 \mathrm{MHz}, \mathrm{CDCl}_{3}\right)$ $\delta 7.73(\mathrm{~d}, J=8.0 \mathrm{~Hz}, 2 \mathrm{H}), 7.36(\mathrm{~d}, J=8.0 \mathrm{~Hz}, 2 \mathrm{H}), 7.32(\mathrm{~d}, J=10.0 \mathrm{~Hz}, 1 \mathrm{H}), 7.24(\mathrm{~d}$, $J=8.4 \mathrm{~Hz}, 1 \mathrm{H}), 6.83(\mathrm{dd}, J=8.4,2.4 \mathrm{~Hz}, 1 \mathrm{H}), 6.75(\mathrm{~d}, J=2.0 \mathrm{~Hz}, 1 \mathrm{H}), 5.78(\mathrm{~d}, J=$ $10.0 \mathrm{~Hz}, 1 \mathrm{H}), 5.10(\mathrm{~s}, 1 \mathrm{H}), 4.40(\mathrm{~s}, 1 \mathrm{H}), 4.32\left(\mathrm{AB}, J_{A B}=13.6 \mathrm{~Hz}, 1 \mathrm{H}\right), 3.84(\mathrm{~s}, 3 \mathrm{H})$, 3.81-3.78 (m, 2H), $3.28(\mathrm{td}, J=12.4,2.8 \mathrm{~Hz}, 1 \mathrm{H}), 2.46(\mathrm{~s}, 3 \mathrm{H}), 2.21(\mathrm{td}, J=13.2,5.2$ $\mathrm{Hz}, 1 \mathrm{H}), 1.89$ (d, $J=14.4 \mathrm{~Hz}, 1 \mathrm{H}) .{ }^{13} \mathrm{C} \mathrm{NMR}\left(100 \mathrm{MHz}, \mathrm{CDCl}_{3}\right) \delta 201.0,161.4,146.1$, 145.1, 143.7, 142.0, 133.8, 131.0, 129.8, 127.7, 123.4, 120.7, 116.7, 115.7, 113.0, 55.6, 54.5, 50.6, 42.1 , 36.4, 21.6; IR (film): $\mathrm{V}_{\max }\left(\mathrm{cm}^{-1}\right)=2980,2954,2917,2848,1647,1621$, $1600,1557,1498,1463,1432,1398,1337,1307,1286,1259,1232,1200,1173,1155$, $1117,1095,1034,945,921,887,844,816,785,753,737,714,685,659,602,545,529$, 491, 466, 423; HRMS (ESI): Exact mass calcd. for $\mathrm{C}_{23} \mathrm{H}_{24} \mathrm{NO}_{4} \mathrm{~S}\left([\mathrm{M}+\mathrm{H}]^{+}\right): 410.1421$. Found: 410.1431.

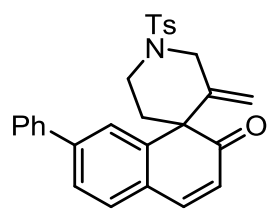

2I: white solid, $88.6 \mathrm{mg}, 97 \%$ yield, m.p. $=140-141{ }^{\circ} \mathrm{C} .{ }^{1} \mathrm{H}$ NMR $\left(400 \mathrm{MHz}, \mathrm{CDCl}_{3}\right) \delta$ $7.72(\mathrm{~d}, J=8.0 \mathrm{~Hz}, 2 \mathrm{H}), 7.62-7.58(\mathrm{~m}, 3 \mathrm{H}), 7.54(\mathrm{~d}, J=7.6 \mathrm{~Hz}, 1 \mathrm{H}), 7.48$ (t, $J=7.6$ $\mathrm{Hz}, 2 \mathrm{H}), 7.42-7.36(\mathrm{~m}, 3 \mathrm{H}), 7.33$ (d, $J=7.6 \mathrm{~Hz}, 2 \mathrm{H}), 5.91(\mathrm{~d}, J=10.0 \mathrm{~Hz}, 1 \mathrm{H}), 5.14(\mathrm{~s}$, $1 \mathrm{H}), 4.48(\mathrm{~s}, 1 \mathrm{H}), 4.34\left(\mathrm{AB}, J_{A B}=13.2 \mathrm{~Hz}, 1 \mathrm{H}\right), 3.80-3.74(\mathrm{~m}, 2 \mathrm{H}), 3.23(\mathrm{td}, J=12.4$, $2.4 \mathrm{~Hz}, 1 \mathrm{H}), 2.38-2.34(\mathrm{~m}, 4 \mathrm{H}), 1.97(\mathrm{~d}, J=14.4 \mathrm{~Hz}, 1 \mathrm{H}) .{ }^{13} \mathrm{C} \mathrm{NMR}\left(100 \mathrm{MHz}, \mathrm{CDCl}_{3}\right)$ $\delta$ 201.0, 144.8, 144.4, 143.7, 143.2, 142.0, 139.8, 133.4, 129.9, 129.8, 129.1, 129.0, 128.6, 128.2, 127.7, 127.2, 126.3, 123.0, 116.9, 54.6, 50.8, 42.1, 36.6, 21.5; IR (film): $\mathrm{V}_{\max }\left(\mathrm{cm}^{-1}\right)=3068,3037,2986,2926,2855,1654,1622,1600,1551,1484,1458,1383$, 1344, 1330, 1306, 1250, 1227, 1205, 1159, 1095, 1024, 1006, 964, 920, 899, 845, 815, 778, 751, 703, 679, 657, 601, 569, 548, 526, 489, 460, 424; HRMS (ESI): Exact mass calcd. for $\mathrm{C}_{28} \mathrm{H}_{26} \mathrm{NO}_{3} \mathrm{~S}\left([\mathrm{M}+\mathrm{H}]^{+}\right)$: 456.1628. Found: 456.1636 . 


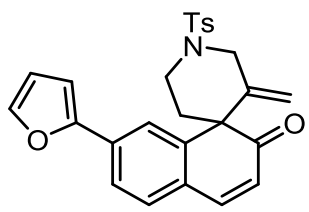

2m: light brown solid, $82.0 \mathrm{mg}, 92 \%$ yield, m.p. $=152-153{ }^{\circ} \mathrm{C} .{ }^{1} \mathrm{H}$ NMR $(400 \mathrm{MHz}$, $\left.\mathrm{CDCl}_{3}\right) \delta 7.72(\mathrm{~s}, 3 \mathrm{H}), 7.61(\mathrm{~d}, J=7.6 \mathrm{~Hz}, 1 \mathrm{H}), 7.54(\mathrm{~s}, 1 \mathrm{H}), 7.38-7.36(\mathrm{~m}, 3 \mathrm{H}), 7.32$ $(\mathrm{d}, J=7.6 \mathrm{~Hz}, 1 \mathrm{H}), 6.79(\mathrm{~s}, 1 \mathrm{H}), 6.53(\mathrm{~s}, 1 \mathrm{H}), 5.88(\mathrm{~d}, J=9.6 \mathrm{~Hz}, 1 \mathrm{H}), 5.13(\mathrm{~s}, 1 \mathrm{H})$, $4.45(\mathrm{~s}, 1 \mathrm{H}), 4.33\left(\mathrm{AB}, J_{A B}=13.2 \mathrm{~Hz}, 1 \mathrm{H}\right), 3.79(\mathrm{~d}, J=9.6 \mathrm{~Hz}, 1 \mathrm{H}), 3.70\left(\mathrm{BA}, J_{B A}=\right.$ $13.2 \mathrm{~Hz}, 1 \mathrm{H}), 3.19$ (t, $J=11.2 \mathrm{~Hz}, 1 \mathrm{H}), 2.45-2.37$ (m, 4H), 1.97 (d, $J=14.0 \mathrm{~Hz}, 1 \mathrm{H}$ ). ${ }^{13} \mathrm{C}$ NMR $\left(100 \mathrm{MHz}, \mathrm{CDCl}_{3}\right) \delta 201.0,152.7,144.8,144.5,143.7,143.1,141.8,133.0$, $132.5,129.9,129.8,129.0,127.8,124.9,122.7,117.0,112.1,107.1,54.4,50.7,42.2$, 36.4, 21.6; IR (film): $v_{\max }\left(\mathrm{cm}^{-1}\right)=3138,2952,2925,2895,2852,1724,1653,1600$, $1544,1506,1491,1464,1441,1395,1369,1348,1333,1305,1281,1254,1229,1212$, 1190, 1162, 1123, 1101, 1087, 1054, 1030, 1015, 956, 912, 886, 852, 814, 748, 707, 680, 659, 597, 554, 541, 521, 488, 452, 413; HRMS (ESI): Exact mass calcd. for $\mathrm{C}_{26} \mathrm{H}_{24} \mathrm{NO}_{4} \mathrm{~S}\left([\mathrm{M}+\mathrm{H}]^{+}\right): 446.1421$. Found: 446.1427.

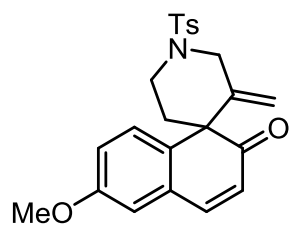

2n: white solid, $80.6 \mathrm{mg}$, 98\% yield, m.p. $=157-159{ }^{\circ} \mathrm{C} .{ }^{1} \mathrm{H}$ NMR $\left(400 \mathrm{MHz}, \mathrm{CDCl}_{3}\right)$ $\delta 7.73(\mathrm{~d}, J=8.0 \mathrm{~Hz}, 2 \mathrm{H}), 7.36(\mathrm{~d}, J=7.6 \mathrm{~Hz}, 2 \mathrm{H}), 7.31(\mathrm{~d}, J=10.0 \mathrm{~Hz}, 1 \mathrm{H}), 7.06(\mathrm{~d}$, $J=8.4 \mathrm{~Hz}, 1 \mathrm{H}), 6.91(\mathrm{~d}, J=7.6 \mathrm{~Hz}, 1 \mathrm{H}), 6.80(\mathrm{~s}, 1 \mathrm{H}), 5.89(\mathrm{~d}, J=10.0 \mathrm{~Hz}, 1 \mathrm{H}), 5.07$ (s, 1H), $4.35(\mathrm{~s}, 1 \mathrm{H}), 4.31\left(\mathrm{AB}, J_{A B}=13.6 \mathrm{~Hz}, 1 \mathrm{H}\right), 3.84(\mathrm{~s}, 3 \mathrm{H}), 3.79-3.75(\mathrm{~m}, 2 \mathrm{H})$, $3.30(\mathrm{t}, J=12.0 \mathrm{~Hz}, 1 \mathrm{H}), 2.47(\mathrm{~s}, 3 \mathrm{H}), 2.23(\mathrm{td}, J=13.2,4.8 \mathrm{~Hz}, 1 \mathrm{H}), 1.86(\mathrm{~d}, J=14.0$ $\mathrm{Hz}, 1 \mathrm{H}) .{ }^{13} \mathrm{C} \mathrm{NMR}\left(100 \mathrm{MHz}, \mathrm{CDCl}_{3}\right) \delta 201.1,158.6,145.1,143.6,141.9,135.5,134.0$, $131.1,130.9,129.8,127.8,123.6,116.7,116.2,113.7,55.4,53.7,50.5,42.3,35.9,21.6$; IR (film): $V_{\max }\left(\mathrm{cm}^{-1}\right)=2953,2926,2851,1656,1599,1566,1501,1459,1435,1397$, $1377,1331,1288,1266,1245,1189,1160,1135,1097,1031,998,949,912,866,815$, 780, 754, 710, 686, 660, 635, 604, 584, 556, 543, 493, 478, 448; HRMS (ESI): Exact mass calcd. for $\mathrm{C}_{23} \mathrm{H}_{24} \mathrm{NO}_{4} \mathrm{~S}\left([\mathrm{M}+\mathrm{H}]^{+}\right)$: 410.1421 . Found: 410.1427 . 


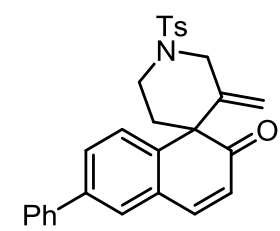

2o: pale white solid, $80.9 \mathrm{mg}, 89 \%$ yield, m.p. $=165-166{ }^{\circ} \mathrm{C} .{ }^{1} \mathrm{H}$ NMR $(400 \mathrm{MHz}$, $\left.\mathrm{CDCl}_{3}\right) \delta 7.74(\mathrm{~d}, J=8.4 \mathrm{~Hz}, 2 \mathrm{H}), 7.61-7.57(\mathrm{~m}, 3 \mathrm{H}), 7.51-7.36(\mathrm{~m}, 7 \mathrm{H}), 7.27(\mathrm{~d}, J=$ $8.0 \mathrm{~Hz}, 1 \mathrm{H}), 5.94(\mathrm{~d}, J=10.0 \mathrm{~Hz}, 1 \mathrm{H}), 5.12(\mathrm{~s}, 1 \mathrm{H}), 4.43(\mathrm{~s}, 1 \mathrm{H}), 4.35\left(\mathrm{AB}, J_{A B}=13.2\right.$ $\mathrm{Hz}, 1 \mathrm{H}), 3.82-3.79(\mathrm{~m}, 2 \mathrm{H}), 3.31(\mathrm{td}, J=12.4,3.2 \mathrm{~Hz}, 1 \mathrm{H}), 2.47(\mathrm{~s}, 3 \mathrm{H}), 2.31(\mathrm{td}, J=$ 14.0, 5.2 Hz, 1H), $1.93(\mathrm{~d}, J=14.0 \mathrm{~Hz}, 1 \mathrm{H}) .{ }^{13} \mathrm{C} \mathrm{NMR}\left(100 \mathrm{MHz}, \mathrm{CDCl}_{3}\right) \delta 200.8$, 145.1, 143.6, 142.6, 141.7, 140.5, 139.5, 134.1, 130.5, 130.3, 129.8, 129.0, 128.8, 127.9, 127.8, 126.9, 123.6, 116.9, 54.1, 50.6, 42.2, 36.0, 21.6; IR (film): $\mathrm{v}_{\max }\left(\mathrm{cm}^{-1}\right)=3032$, 2966, 2925, 2891, 2118, 1646, 1596, 1557, 1486, 1454, 1374, 1342, 1314, 1291, 1253 , 1226, 1194, 1156, 1117, 1096, 1029, 998, 960, 948, 912, 898, 876, 853, 813, 762, 690, 655, 600, 557, 544, 532, 492, 465, 444; HRMS (ESI): Exact mass calcd. for $\mathrm{C}_{28} \mathrm{H}_{26} \mathrm{NO}_{3} \mathrm{~S}\left([\mathrm{M}+\mathrm{H}]^{+}\right): 456.1628$. Found: 456.1637 .

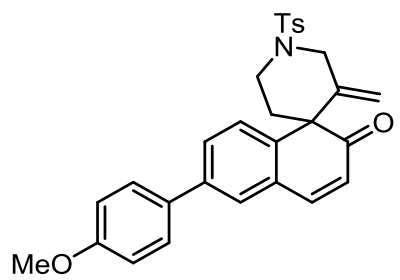

2p: white solid, $90.0 \mathrm{mg}$, 93\% yield, m.p. $=223-224{ }^{\circ} \mathrm{C} .{ }^{1} \mathrm{H}$ NMR $\left(400 \mathrm{MHz}, \mathrm{CDCl}_{3}\right)$ $\delta 7.74(\mathrm{~d}, J=8.0 \mathrm{~Hz}, 2 \mathrm{H}), 7.55-7.53(\mathrm{~m}, 3 \mathrm{H}), 7.46(\mathrm{~s}, 1 \mathrm{H}), 7.42(\mathrm{~d}, J=9.6 \mathrm{~Hz}, 1 \mathrm{H})$, $7.37(\mathrm{~d}, J=8.0 \mathrm{~Hz}, 2 \mathrm{H}), 7.25(\mathrm{~d}, J=8.8 \mathrm{~Hz}, 1 \mathrm{H}), 7.00(\mathrm{~d}, J=8.4 \mathrm{~Hz}, 2 \mathrm{H}), 5.93(\mathrm{~d}, J=$ $10.0 \mathrm{~Hz}, 1 \mathrm{H}), 5.12(\mathrm{~s}, 1 \mathrm{H}), 4.43(\mathrm{~s}, 1 \mathrm{H}), 4.34\left(\mathrm{AB}, J_{A B}=13.6 \mathrm{~Hz}, 1 \mathrm{H}\right), 3.87(\mathrm{~s}, 3 \mathrm{H})$, 3.81-3.78 (m, 2H), $3.30(\mathrm{td}, J=12.4,2.4 \mathrm{~Hz}, 1 \mathrm{H}), 2.48(\mathrm{~s}, 3 \mathrm{H}), 2.31(\mathrm{td}, J=12.8,4.8$ $\mathrm{Hz}, 1 \mathrm{H}), 1.92(\mathrm{~d}, J=14.4 \mathrm{~Hz}, 1 \mathrm{H}) .{ }^{13} \mathrm{C} \mathrm{NMR}\left(100 \mathrm{MHz}, \mathrm{CDCl}_{3}\right) \delta 200.9,159.6,145.2$, $143.6,141.9,141.7,140.1,134.0,132.0,130.4,130.2$, 129.8, 128.4, 128.0, 127.8, 127.3, 123.5, 116.8, 114.4, 55.4, 54.1, 50.6, 42.2, 36.0, 21.6; IR (film): $\mathrm{V}_{\max }\left(\mathrm{cm}^{-1}\right)=2963$, 2923, 2886, 2851, 2808, 1652, 1599, 1557, 1519, 1491, 1459, 1435, 1374, 1332, 1305, $1285,1246,1229,1182,1161,1121,1098,1042,1022,999,949,911,879,858,814$, 758, 708, 690, 661, 628, 605, 590, 559, 543, 523; HRMS (ESI): Exact mass calcd. for 
$\mathrm{C}_{29} \mathrm{H}_{28} \mathrm{NO}_{4} \mathrm{~S}\left([\mathrm{M}+\mathrm{H}]^{+}\right): 486.1734$. Found: 486.1745 .

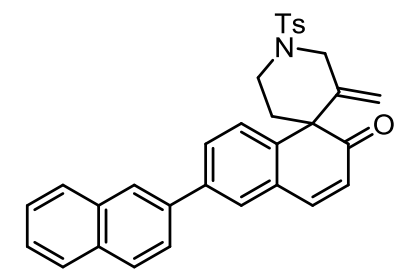

2q: white solid, $100.1 \mathrm{mg}, 99 \%$ yield, m.p. $=166-167{ }^{\circ} \mathrm{C} .{ }^{1} \mathrm{H} \mathrm{NMR}\left(400 \mathrm{MHz}, \mathrm{CDCl}_{3}\right)$ $\delta 8.05(\mathrm{~s}, 1 \mathrm{H}), 7.94-7.90(\mathrm{~m}, 2 \mathrm{H}), 7.87(\mathrm{~d}, J=7.2 \mathrm{~Hz}, 1 \mathrm{H}), 7.75-7.73(\mathrm{~m}, 3 \mathrm{H}), 7.68(\mathrm{~d}$, $J=8.0 \mathrm{~Hz}, 1 \mathrm{H}), 7.62(\mathrm{~s}, 1 \mathrm{H}), 7.54-7.48(\mathrm{~m}, 2 \mathrm{H}), 7.44$ (d, $J=10.0 \mathrm{~Hz}, 1 \mathrm{H}), 7.34$ (d, $J$ $=8.0 \mathrm{~Hz}, 2 \mathrm{H}), 7.28(\mathrm{~d}, J=8.4 \mathrm{~Hz}, 1 \mathrm{H}), 5.94(\mathrm{~d}, J=10.0 \mathrm{~Hz}, 1 \mathrm{H}), 5.12(\mathrm{~s}, 1 \mathrm{H}), 4.44(\mathrm{~s}$, $1 \mathrm{H}), 4.36\left(\mathrm{AB}, J_{A B}=13.6 \mathrm{~Hz}, 1 \mathrm{H}\right), 3.82-3.78(\mathrm{~m}, 2 \mathrm{H}), 3.29(\mathrm{td}, J=11.6,1.6 \mathrm{~Hz}, 1 \mathrm{H})$, $2.45(\mathrm{~s}, 3 \mathrm{H}), 2.30(\mathrm{td}, J=13.6,4.8 \mathrm{~Hz}, 1 \mathrm{H}), 1.92(\mathrm{~d}, J=14.0 \mathrm{~Hz}, 1 \mathrm{H}) .{ }^{13} \mathrm{C} \mathrm{NMR}(100$ $\left.\mathrm{MHz}, \mathrm{CDCl}_{3}\right) \delta 200.8,145.2,143.7,142.7,141.7,140.4,136.8,133.9,133.6,132.8$, 130.6, 130.3, 129.9, 129.0, 128.8, 128.3, 128.1, 127.8, 127.7, 126.6, 126.4, 125.8, 125.0, 123.6, 117.0, 54.1, 50.7, 42.2, 36.1, 21.7; IR (film): $\mathrm{V}_{\max }\left(\mathrm{cm}^{-1}\right)=3053,2959,2921$, 2851, 1648, 1595, 1560, 1493, 1459, 1386, 1342, 1330, 1306, 1284, 1251, 1218, 1159 , 1129, 1092, 1029, 1001, 962, 929, 908, 863, 817, 748, 709, 693, 660, 604, 578, 543, 471, 452; HRMS (ESI): Exact mass calcd. for $\mathrm{C}_{32} \mathrm{H}_{28} \mathrm{NO}_{3} \mathrm{~S}\left([\mathrm{M}+\mathrm{H}]^{+}\right): 506.1784$. Found: 506.1785 .

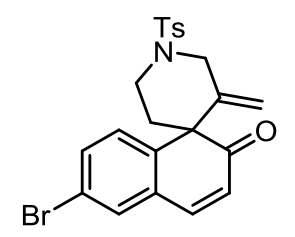

2r: white solid, $76.6 \mathrm{mg}, 84 \%$ yield, m.p. $=140-142{ }^{\circ} \mathrm{C} .{ }^{1} \mathrm{H} \mathrm{NMR}\left(400 \mathrm{MHz}, \mathrm{CDCl}_{3}\right)$ $\delta 7.72(\mathrm{~d}, J=8.0 \mathrm{~Hz}, 2 \mathrm{H}), 7.48-7.4(\mathrm{~m}, 2 \mathrm{H}), 7.36(\mathrm{~d}, J=8.0 \mathrm{~Hz}, 2 \mathrm{H}), 7.29(\mathrm{~d}, J=10.0$ Hz, 1H), 7.07 (d, $J=8.4 \mathrm{~Hz}, 1 \mathrm{H}), 5.93$ (d, $J=9.6 \mathrm{~Hz}, 1 \mathrm{H}), 5.11(\mathrm{~s}, 1 \mathrm{H}), 4.35$ (s, 1H), $4.33\left(\mathrm{AB}, J_{A B}=16.4 \mathrm{~Hz}, 1 \mathrm{H}\right), 3.81-3.75(\mathrm{~m}, 1 \mathrm{H}), 3.77\left(\mathrm{BA}, J_{B A}=13.6 \mathrm{~Hz}, 1 \mathrm{H}\right), 3.26$ $(\mathrm{td}, J=12.4,2.8 \mathrm{~Hz}, 1 \mathrm{H}), 2.47(\mathrm{~s}, 3 \mathrm{H}), 2.21(\mathrm{td}, J=13.6,4.8 \mathrm{~Hz}, 1 \mathrm{H}), 1.88(\mathrm{~d}, J=14.0$ $\mathrm{Hz}, 1 \mathrm{H}) .{ }^{13} \mathrm{C} \mathrm{NMR}\left(100 \mathrm{MHz}, \mathrm{CDCl}_{3}\right) \delta 200.1,143.7,143.6,142.6,141.2,133.9,132.9$, 132.0, 131.7, 131.5, 129.8, 127.8, 124.3, 121.3, 117.0, 54.1, 50.5, 42.0, 35.9, 21.6; IR (film): $\mathrm{v}_{\max }\left(\mathrm{cm}^{-1}\right)=2214,2096,1658,1595,1552,1485,1459,1438,1369,1345,1322$, 1303, 1289, 1269, 1249, 1230, 1211, 1159, 1122, 1098, 1047, 1023, 994, 948, 931, 913, 
$866,841,816,799,754,732,709,678,656,606,543,525,480,459,436,412$; HRMS

(ESI): Exact mass calcd. for $\mathrm{C}_{22} \mathrm{H}_{21} \mathrm{BrNO}_{3} \mathrm{~S}\left([\mathrm{M}+\mathrm{H}]^{+}\right)$: 458.0420. Found: 458.0429 .

\subsection{Transformation of products}

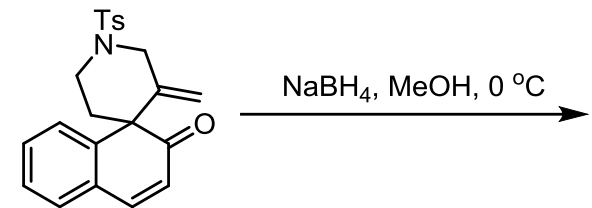

2a

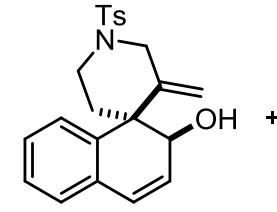

$3 a$

$77 \%$ combined yield, $2.3: 1 \mathrm{dr}$

$\mathrm{NaBH}_{4}(15.1 \mathrm{mg}, 0.4 \mathrm{mmol}, 2.0$ equiv) was added to a solution of $2 \mathbf{a}(75.9 \mathrm{mg}$, $0.2 \mathrm{mmol}, 1.0$ equiv) in $\mathrm{MeOH}(5.0 \mathrm{~mL})$ at $0{ }^{\circ} \mathrm{C}$. The reaction mixture was stirred at room temperature. After the reaction was complete (monitored by TLC), the crude was quenched by saturated $\mathrm{NH}_{4} \mathrm{Cl}$ aqueous solution $(10.0 \mathrm{~mL})$. Methanol was evaporated and the mixture was extracted with EtOAc $(10.0 \mathrm{~mL}$ x 3). The organic layer was washed with brine, dried over anhydrous $\mathrm{Na}_{2} \mathrm{SO}_{4}$ and filtered. After the solvent was concentrated under reduced pressure, the crude product was purified by silica gel column chromatography $(\mathrm{PE} / \mathrm{EtOAc}=5: 1-3: 1)$ to afford the desired product $\mathbf{3 a} / \mathbf{3 a}$ ' The analytical data of the product are summarized below.

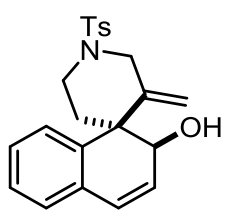

3a: white solid, $40.4 \mathrm{mg}$, 53\% yield, m.p. $=66-67{ }^{\circ} \mathrm{C} .{ }^{1} \mathrm{H}$ NMR $\left(400 \mathrm{MHz}, \mathrm{CDCl}_{3}\right) \delta$ $7.70(\mathrm{~d}, J=8.0 \mathrm{~Hz}, 2 \mathrm{H}), 7.36(\mathrm{~d}, J=8.0 \mathrm{~Hz}, 2 \mathrm{H}), 7.17(\mathrm{t}, J=6.8 \mathrm{~Hz}, 1 \mathrm{H}), 7.12-7.08$ (m, 2H), $7.03(\mathrm{~d}, J=7.6 \mathrm{~Hz}, 1 \mathrm{H}), 6.54(\mathrm{~d}, J=9.6 \mathrm{~Hz}, 1 \mathrm{H}), 6.05(\mathrm{dd}, J=9.2,5.6 \mathrm{~Hz}$, $1 \mathrm{H}), 5.46(\mathrm{~s}, 1 \mathrm{H}), 5.09(\mathrm{~s}, 1 \mathrm{H}), 4.31\left(\mathrm{AB}, J_{A B}=14.0 \mathrm{~Hz}, 1 \mathrm{H}\right), 3.94(\mathrm{~d}, J=5.2 \mathrm{~Hz}, 1 \mathrm{H})$, $3.48\left(\mathrm{BA}, J_{B A}=14.0 \mathrm{~Hz}, 1 \mathrm{H}\right), 3.45-3.39(\mathrm{~m}, 1 \mathrm{H}), 2.92-2.86(\mathrm{~m}, 1 \mathrm{H}), 2.47(\mathrm{~s}, 3 \mathrm{H}), 1.84-$ $1.66(\mathrm{~m}, 3 \mathrm{H}) .{ }^{13} \mathrm{C} \mathrm{NMR}\left(100 \mathrm{MHz}, \mathrm{CDCl}_{3}\right) \delta 143.8,139.4,138.1,133.5,131.7,129.9$, $129.8,128.33,128.26,128.1,127.9,127.0,125.0,119.0,64.9,50.4,48.7,42.4,34.0$, 21.6; IR (film): $V_{\max }\left(\mathrm{cm}^{-1}\right)=3502,3063,3026,2954,2922,2855,1918,1719,1643$, 1597, 1487, 1450, 1400, 1342, 1305, 1288, 1218, 1185, 1156, 1120, 1090, 1037, 1003, 956, 915, 815, 794, 752, 708, 661, 601, 545, 469; HRMS (ESI): Exact mass calcd. for 
$\mathrm{C}_{22} \mathrm{H}_{24} \mathrm{NO}_{3} \mathrm{~S}\left([\mathrm{M}+\mathrm{H}]^{+}\right): 382.1471$. Found: 382.1475 .

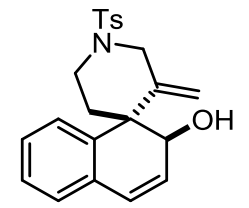

3a': white solid, $18.6 \mathrm{mg}, 24 \%$ yield, m.p. $=59-60{ }^{\circ} \mathrm{C} .{ }^{1} \mathrm{H}$ NMR $\left(400 \mathrm{MHz}, \mathrm{CDCl}_{3}\right) \delta$ $7.70(\mathrm{~d}, J=8.4 \mathrm{~Hz}, 2 \mathrm{H}), 7.35(\mathrm{~d}, J=8.0 \mathrm{~Hz}, 2 \mathrm{H}), 7.24-7.16(\mathrm{~m}, 2 \mathrm{H}), 7.10-7.06(\mathrm{~m}, 2 \mathrm{H})$, $6.44(\mathrm{~d}, J=9.6 \mathrm{~Hz}, 1 \mathrm{H}), 5.93(\mathrm{dd}, J=9.6,5.2 \mathrm{~Hz}, 1 \mathrm{H}), 4.97(\mathrm{~s}, 1 \mathrm{H}), 4.32-4.30(\mathrm{~m}, 1 \mathrm{H})$, $4.26(\mathrm{~s}, 1 \mathrm{H}), 4.15\left(\mathrm{AB}, J_{A B}=13.6 \mathrm{~Hz}, 1 \mathrm{H}\right), 3.64-3.59(\mathrm{~m}, 1 \mathrm{H}), 3.44\left(\mathrm{BA}, J_{B A}=13.2 \mathrm{~Hz}\right.$, 1H), 3.06-3.00 (m, 1H), $2.47(\mathrm{~s}, 3 \mathrm{H}), 2.39-2.34(\mathrm{~m}, 1 \mathrm{H}), 2.28-2.21(\mathrm{~m}, 1 \mathrm{H}), 1.51(\mathrm{~d}, J$ $=7.2 \mathrm{~Hz}, 1 \mathrm{H}) .{ }^{13} \mathrm{C} \mathrm{NMR}\left(100 \mathrm{MHz}, \mathrm{CDCl}_{3}\right) \delta 143.7,143.4,137.3,133.3,132.8,129.8$, $129.5,128.5,127.8,127.5,127.4,127.4,126.8,115.2,67.2,50.1,47.3,42.2,27.7,21.6$; IR (film): $V_{\max }\left(\mathrm{cm}^{-1}\right)=3521,3029,2973,2926,2866,2842,1641,1597,1471,1450$, 1402, 1343, 1306, 1289, 1235, 1216, 1184, 1159, 1118, 1091, 1052, 1038, 996, 959, 916, 882, 818, 790, 754, 710, 663, 627, 607, 584, 547, 492, 445; HRMS (ESI): Exact mass calcd. for $\mathrm{C}_{22} \mathrm{H}_{24} \mathrm{NO}_{3} \mathrm{~S}\left([\mathrm{M}+\mathrm{H}]^{+}\right)$: 382.1471. Found: 382.1473 .

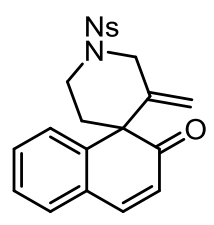

$2 \mathrm{e}$

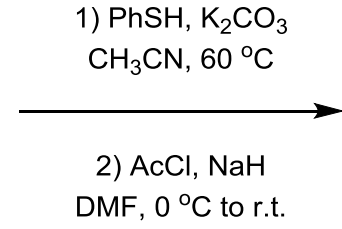

DMF, $0^{\circ} \mathrm{C}$ to r.t.

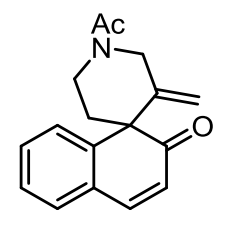

$3 e$

$78 \%$ yield for 2 steps

$\mathrm{PhSH}$ (66.1 mg, $0.6 \mathrm{mmol}, 2.0$ equiv) was added to a solution of $\mathbf{2 e}(123.1 \mathrm{mg}, 0.3$ mmol, 1.0 equiv) and $\mathrm{K}_{2} \mathrm{CO}_{3}\left(82.9 \mathrm{mg}, 0.6 \mathrm{mmol}, 2.0\right.$ equiv) in $\mathrm{CH}_{3} \mathrm{CN}(3 \mathrm{~mL})$ at room temperature. Then the reaction mixture was stirred at $60{ }^{\circ} \mathrm{C}$ for $12 \mathrm{~h}$. The reaction system was filtered through celite and washed by EtOAc $(30 \mathrm{~mL})$. The mixture was concentrated in vacuo to afford the amine.

$\mathrm{NaH}$ (20 mg, $0.6 \mathrm{mmol}, 2.0$ equiv, $60 \%$ dispersion in mineral oil) was added to the solution of the above obtained amine in $\operatorname{DMF}(3.0 \mathrm{~mL})$ at $0{ }^{\circ} \mathrm{C}$, then the reaction mixture was stirred at $0{ }^{\circ} \mathrm{C}$ for $30 \mathrm{~min}$. $\mathrm{AcCl}(39 \mathrm{mg}, 35 \mu \mathrm{L}, 0.45 \mathrm{mmol}, 1.5$ equiv) was added dropwise. The reaction mixture was stirred at room temperature. After the reaction was complete (monitored by TLC), the crude reaction mixture was quenched by water ( 5 $\mathrm{mL})$. The mixture was extracted with EtOAc $(10.0 \mathrm{~mL}$ x 3$)$. The combined organic layer 
was washed with brine, dried over anhydrous $\mathrm{Na}_{2} \mathrm{SO}_{4}$ and filtered. After the solvent was concentrated under reduced pressure, the crude product was purified by silica gel column chromatography $(\mathrm{PE} / \mathrm{EtOAc}=5: 1-1: 1)$ to afford the desired product 3e. The analytical data of the product are summarized below.

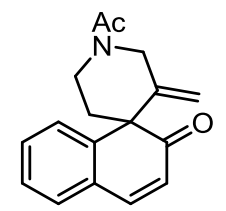

3e: yellow oil, $62.8 \mathrm{mg}, 78 \%$ yield for 2 steps. ${ }^{1} \mathrm{H}$ NMR (400 MHz, $\left.\mathrm{CDCl}_{3}\right) \delta$ 7.41-7.26 (m, 5H), $6.02(\mathrm{~d}, J=9.6 \mathrm{~Hz}, 1 \mathrm{H}), 5.09(\mathrm{~d}, J=24.0 \mathrm{~Hz}, 1 \mathrm{H}), 4.93(\mathrm{~d}, J=15.2 \mathrm{~Hz}, 0.5 \mathrm{H})$, $4.47(\mathrm{~d}, J=14.4 \mathrm{~Hz}, 0.5 \mathrm{H}), 4.36(\mathrm{~d}, J=8.4 \mathrm{~Hz}, 1 \mathrm{H}), 4.27(\mathrm{~d}, J=13.2 \mathrm{~Hz}, 0.5 \mathrm{H}), 4.20$ $(\mathrm{d}, J=14.8 \mathrm{~Hz}, 0.5 \mathrm{H}), 3.99(\mathrm{~d}, J=14.8 \mathrm{~Hz}, 0.5 \mathrm{H}), 3.95-3.89(\mathrm{~m}, 0.5 \mathrm{H}), 3.69-3.54(\mathrm{~m}$, 1H), 2.16-2.13 (m, 4H), 2.08-2.06 (m, 1H). $\left.{ }^{13} \mathrm{C} \mathrm{NMR} \mathrm{(100} \mathrm{MHz,} \mathrm{CDCl}_{3}\right) \delta$ 201.1, 169.0, 145.0, 144.9, 144.4, 144.3, 143.4, 142.8, 130.20, 130.17, 130.00, 129.95, 129.5, 129.4, $129.1,127.5,123.5,123.3,116.6,115.9,55.5,55.4,51.3,45.9,42.3,38.2,36.6,36.3$, 21.6, 21.4. ${ }^{1} \mathrm{H}$ NMR $\left(600 \mathrm{MHz}, d_{6}\right.$-DMSO, $\left.80{ }^{\circ} \mathrm{C}\right) \delta 7.63(\mathrm{~d}, J=9.6 \mathrm{~Hz}, 1 \mathrm{H}), 7.49-7.47$ (m, 1H), 7.46-7.43 (m, 1H), 7.37-7.35 (m, 1H), 7.33-7.30 (m, 1H), $6.01(\mathrm{~d}, J=10.2 \mathrm{~Hz}$, $1 \mathrm{H}), 5.11(\mathrm{~d}, J=58.8 \mathrm{~Hz}, 1 \mathrm{H}), 4.65(\mathrm{~s}, 0.5 \mathrm{H}), 4.33-4.25(\mathrm{~m}, 2 \mathrm{H}), 3.95-3.91(\mathrm{~m}, 1 \mathrm{H})$, 3.75-3.71 (m, 1H), 3.53-3.48 (m, 0.5H), 2.18-1.99 (m, 5H). ${ }^{13} \mathrm{C}$ NMR (150 MHz, $d_{6^{-}}$ DMSO, $\left.80{ }^{\circ} \mathrm{C}\right) \delta 199.8,167.9,144.4,144.1,143.6,129.6,129.3,129.0,128.2,126.9$, 122.5, 114.5, 54.9, 49.6, 44.9, 41.3, 37.4, 35.1, 20.6; IR (film): $\mathrm{V}_{\max }\left(\mathrm{cm}^{-1}\right)=3471,2922$, 2853, 2386, 1617, 1564, 1423, 1399, 1362, 1343, 1304, 1244, 1194, 1161, 1132, 1091, 1025, 1007, 983, 912, 879, 829, 754, 701, 657, 599, 536, 471; HRMS (ESI): Exact mass calcd. for $\mathrm{C}_{17} \mathrm{H}_{18} \mathrm{NO}_{2}\left([\mathrm{M}+\mathrm{H}]^{+}\right)$: 268.1332. Found: 268.1339 .

\section{References}

(1) Langlois, M.; Bremont, B.; Shen, S.; Poncet, A.; Andrieux, J.; Sicsic, S.; Serraz, I. M.-Allainmat, M.; Renard, P.; Delagrange, P. J. Med. Chem. 1995, 38, 2050.

(2) Ding, L.; You, S.-L. Org. Lett. 2018, 20, 6206.

\section{X-Ray crystal data of $2 a$ (The crystal was obtained by slow evaporation of $2 a$}




\section{in DCM) (CCDC 1993867)}
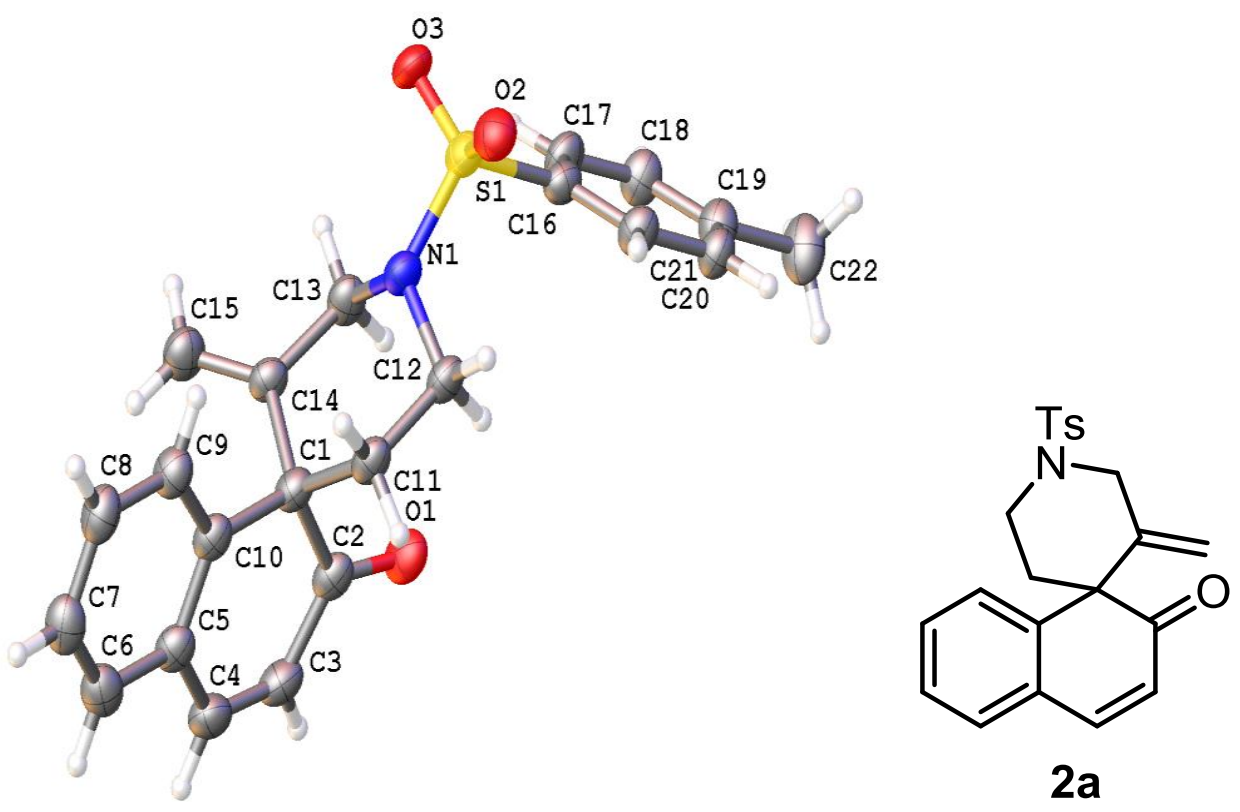

Table S1. Crystal data and structure refinement for mj118252_0m.

Identification code

Empirical formula

Formula weight

Temperature

Wavelength

Crystal system

Space group

Unit cell dimensions

Volume

$\mathrm{Z}$

Density (calculated)

Absorption coefficient

$\mathrm{F}(000)$

Crystal size

Theta range for data collection

Index ranges

Reflections collected

Independent reflections

Completeness to theta $=53.594^{\circ}$

Absorption correction mj118252_0m

C22 H21 N O3 S

379.46

$200 \mathrm{~K}$

$1.34139 \AA$

Orthorhombic

Pbca

$\mathrm{a}=12.314(4) \AA$

$\alpha=90^{\circ}$.

$\mathrm{b}=13.111(4) \AA$

$\beta=90^{\circ}$.

$c=23.197(8) \AA$

$\gamma=90^{\circ}$.

3745(2) $\AA^{3}$

8

$1.346 \mathrm{Mg} / \mathrm{m}^{3}$

$1.115 \mathrm{~mm}^{-1}$

1600

$0.12 \times 0.1 \times 0.01 \mathrm{~mm}^{3}$

4.556 to $56.033^{\circ}$.

$-15<=\mathrm{h}<=12,-9<=\mathrm{k}<=16,-28<=\mathrm{l}<=23$

19215

$3551[\mathrm{R}($ int $)=0.1004]$

$97.1 \%$

Semi-empirical from equivalents 
Max. and min. transmission

Refinement method

Data / restraints / parameters

Goodness-of-fit on $\mathrm{F}^{2}$

Final $\mathrm{R}$ indices [I $>2 \operatorname{sigma}(\mathrm{I})]$

$\mathrm{R}$ indices (all data)

Extinction coefficient

Largest diff. peak and hole
0.7508 and 0.4653

Full-matrix least-squares on $\mathrm{F}^{2}$

3551 / 0 / 246

0.978

$\mathrm{R} 1=0.0758, \mathrm{wR} 2=0.2001$

$\mathrm{R} 1=0.1274, \mathrm{wR} 2=0.2459$

$0.0045(7)$

0.416 and -0.457 e. $\AA^{-3}$

Table S2. Atomic coordinates $\quad\left(x 10^{4}\right)$ and equivalent isotropic displacement parameters $\left(\AA^{2} \mathrm{x}\right.$ $\left.10^{3}\right)$

for mj118252_0m. $\quad \mathrm{U}(\mathrm{eq})$ is defined as one third of the trace of the orthogonalized $\mathrm{U}^{\mathrm{ij}}$ tensor.

\begin{tabular}{|c|c|c|c|c|}
\hline & $\mathrm{x}$ & $\mathrm{y}$ & $\mathrm{z}$ & $\mathrm{U}(\mathrm{eq})$ \\
\hline$S(1)$ & $2206(1)$ & $3016(1)$ & $3502(1)$ & $58(1)$ \\
\hline $\mathrm{O}(1)$ & $4566(2)$ & $3633(2)$ & $5168(1)$ & $70(1)$ \\
\hline $\mathrm{O}(2)$ & 1991(2) & $3829(2)$ & $3104(1)$ & $69(1)$ \\
\hline $\mathrm{O}(3)$ & $1413(2)$ & $2241(2)$ & $3587(1)$ & $66(1)$ \\
\hline $\mathrm{N}(1)$ & $2415(2)$ & $3550(2)$ & $4126(1)$ & $53(1)$ \\
\hline $\mathrm{C}(1)$ & $2844(3)$ & $4485(3)$ & $5251(2)$ & $51(1)$ \\
\hline$C(2)$ & $3952(3)$ & $4120(3)$ & $5478(2)$ & $54(1)$ \\
\hline $\mathrm{C}(3)$ & $4253(3)$ & $4356(3)$ & $6075(2)$ & $63(1)$ \\
\hline $\mathrm{C}(4)$ & $3697(3)$ & $5018(3)$ & $6389(2)$ & $64(1)$ \\
\hline$C(5)$ & $2715(3)$ & $5511(3)$ & $6187(2)$ & $55(1)$ \\
\hline$C(6)$ & $2184(3)$ & $6218(3)$ & $6534(2)$ & $65(1)$ \\
\hline$C(7)$ & $1222(4)$ & $6669(3)$ & $6357(2)$ & $68(1)$ \\
\hline$C(8)$ & $775(3)$ & $6376(3)$ & $5836(2)$ & $67(1)$ \\
\hline $\mathrm{C}(9)$ & $1295(3)$ & $5681(3)$ & $5491(2)$ & $61(1)$ \\
\hline $\mathrm{C}(10)$ & $2273(2)$ & $5238(3)$ & $5650(2)$ & $52(1)$ \\
\hline $\mathrm{C}(11)$ & $3031(3)$ & $5026(3)$ & $4659(2)$ & $55(1)$ \\
\hline$C(12)$ & $3270(3)$ & $4332(3)$ & $4155(2)$ & $54(1)$ \\
\hline$C(13)$ & $2426(3)$ & $2889(3)$ & $4638(2)$ & $59(1)$ \\
\hline$C(14)$ & 2167(3) & $3523(3)$ & $5169(2)$ & $50(1)$ \\
\hline$C(15)$ & $1420(3)$ & $3201(3)$ & $5538(2)$ & $69(1)$ \\
\hline$C(16)$ & $3421(3)$ & $2437(3)$ & $3276(2)$ & $53(1)$ \\
\hline$C(17)$ & $3628(3)$ & $1426(3)$ & $3396(2)$ & $62(1)$ \\
\hline $\mathrm{C}(18)$ & $4565(3)$ & $979(3)$ & $3210(2)$ & $63(1)$ \\
\hline
\end{tabular}




$\begin{array}{lllll}\mathrm{C}(19) & 5336(3) & 1516(3) & 2911(2) & 60(1) \\ \mathrm{C}(20) & 5145(3) & 2537(3) & 2796(2) & 57(1) \\ \mathrm{C}(21) & 4183(3) & 2995(3) & 2980(2) & 58(1) \\ \mathrm{C}(22) & 6379(4) & 1035(4) & 2705(2) & 84(1)\end{array}$

Table S3. Bond lengths $[\AA]$ and angles $\left[{ }^{\circ}\right]$ for mj118252_0m.

\begin{tabular}{ll}
\hline $\mathrm{S}(1)-\mathrm{O}(2)$ & $1.434(3)$ \\
$\mathrm{S}(1)-\mathrm{O}(3)$ & $1.423(3)$ \\
$\mathrm{S}(1)-\mathrm{N}(1)$ & $1.629(3)$ \\
$\mathrm{S}(1)-\mathrm{C}(16)$ & $1.758(4)$ \\
$\mathrm{O}(1)-\mathrm{C}(2)$ & $1.223(4)$ \\
$\mathrm{N}(1)-\mathrm{C}(12)$ & $1.471(4)$ \\
$\mathrm{N}(1)-\mathrm{C}(13)$ & $1.471(5)$ \\
$\mathrm{C}(1)-\mathrm{C}(2)$ & $1.539(5)$ \\
$\mathrm{C}(1)-\mathrm{C}(10)$ & $1.525(5)$ \\
$\mathrm{C}(1)-\mathrm{C}(11)$ & $1.563(5)$ \\
$\mathrm{C}(1)-\mathrm{C}(14)$ & $1.525(5)$ \\
$\mathrm{C}(2)-\mathrm{C}(3)$ & $1.466(5)$ \\
$\mathrm{C}(3)-\mathrm{H}(3)$ & 0.9500 \\
$\mathrm{C}(3)-\mathrm{C}(4)$ & $1.324(5)$ \\
$\mathrm{C}(4)-\mathrm{H}(4)$ & 0.9500 \\
$\mathrm{C}(4)-\mathrm{C}(5)$ & $1.449(5)$ \\
$\mathrm{C}(5)-\mathrm{C}(6)$ & $1.391(5)$ \\
$\mathrm{C}(5)-\mathrm{C}(10)$ & $1.405(5)$ \\
$\mathrm{C}(6)-\mathrm{H}(6)$ & 0.9500 \\
$\mathrm{C}(6)-\mathrm{C}(7)$ & $1.386(6)$ \\
$\mathrm{C}(7)-\mathrm{H}(7)$ & 0.9500 \\
$\mathrm{C}(7)-\mathrm{C}(8)$ & $1.384(6)$ \\
$\mathrm{C}(8)-\mathrm{H}(8)$ & 0.9500 \\
$\mathrm{C}(8)-\mathrm{C}(9)$ & $1.370(5)$ \\
$\mathrm{C}(9)-\mathrm{H}(9)$ & 0.9500 \\
$\mathrm{C}(9)-\mathrm{C}(10)$ & $1.386(4)$ \\
$\mathrm{C}(11)-\mathrm{H}(11 \mathrm{~A})$ & 0.9900 \\
$\mathrm{C}(11)-\mathrm{H}(11 \mathrm{~B})$ & 0.9900 \\
$\mathrm{C}(11)-\mathrm{C}(12)$ & $1.512(5)$ \\
$\mathrm{C}(12)-\mathrm{H}(12 \mathrm{~A})$ & 0.9900 \\
&
\end{tabular}




\begin{tabular}{|c|c|}
\hline $\mathrm{C}(12)-\mathrm{H}(12 \mathrm{~B})$ & 0.9900 \\
\hline $\mathrm{C}(13)-\mathrm{H}(13 \mathrm{~A})$ & 0.9900 \\
\hline $\mathrm{C}(13)-\mathrm{H}(13 \mathrm{~B})$ & 0.9900 \\
\hline$C(13)-C(14)$ & $1.519(5)$ \\
\hline $\mathrm{C}(14)-\mathrm{C}(15)$ & $1.326(5)$ \\
\hline $\mathrm{C}(15)-\mathrm{H}(15 \mathrm{~A})$ & 0.9500 \\
\hline $\mathrm{C}(15)-\mathrm{H}(15 \mathrm{~B})$ & 0.9500 \\
\hline$C(16)-C(17)$ & $1.378(5)$ \\
\hline$C(16)-C(21)$ & $1.375(5)$ \\
\hline $\mathrm{C}(17)-\mathrm{H}(17)$ & 0.9500 \\
\hline $\mathrm{C}(17)-\mathrm{C}(18)$ & $1.363(5)$ \\
\hline $\mathrm{C}(18)-\mathrm{H}(18)$ & 0.9500 \\
\hline $\mathrm{C}(18)-\mathrm{C}(19)$ & $1.372(5)$ \\
\hline $\mathrm{C}(19)-\mathrm{C}(20)$ & $1.385(5)$ \\
\hline $\mathrm{C}(19)-\mathrm{C}(22)$ & $1.508(5)$ \\
\hline $\mathrm{C}(20)-\mathrm{H}(20)$ & 0.9500 \\
\hline$C(20)-C(21)$ & $1.394(5)$ \\
\hline $\mathrm{C}(21)-\mathrm{H}(21)$ & 0.9500 \\
\hline $\mathrm{C}(22)-\mathrm{H}(22 \mathrm{~A})$ & 0.9800 \\
\hline $\mathrm{C}(22)-\mathrm{H}(22 \mathrm{~B})$ & 0.9800 \\
\hline $\mathrm{C}(22)-\mathrm{H}(22 \mathrm{C})$ & 0.9800 \\
\hline $\mathrm{O}(2)-\mathrm{S}(1)-\mathrm{N}(1)$ & $106.39(16)$ \\
\hline $\mathrm{O}(2)-\mathrm{S}(1)-\mathrm{C}(16)$ & $106.66(17)$ \\
\hline $\mathrm{O}(3)-\mathrm{S}(1)-\mathrm{O}(2)$ & $119.64(16)$ \\
\hline $\mathrm{O}(3)-\mathrm{S}(1)-\mathrm{N}(1)$ & $106.91(15)$ \\
\hline $\mathrm{O}(3)-\mathrm{S}(1)-\mathrm{C}(16)$ & $108.46(17)$ \\
\hline $\mathrm{N}(1)-\mathrm{S}(1)-\mathrm{C}(16)$ & $108.36(16)$ \\
\hline $\mathrm{C}(12)-\mathrm{N}(1)-\mathrm{S}(1)$ & $116.9(2)$ \\
\hline $\mathrm{C}(12)-\mathrm{N}(1)-\mathrm{C}(13)$ & $111.6(3)$ \\
\hline $\mathrm{C}(13)-\mathrm{N}(1)-\mathrm{S}(1)$ & $117.9(2)$ \\
\hline$C(2)-C(1)-C(11)$ & 108.2(3) \\
\hline $\mathrm{C}(10)-\mathrm{C}(1)-\mathrm{C}(2)$ & $113.8(3)$ \\
\hline $\mathrm{C}(10)-\mathrm{C}(1)-\mathrm{C}(11)$ & $107.9(3)$ \\
\hline $\mathrm{C}(14)-\mathrm{C}(1)-\mathrm{C}(2)$ & $105.7(3)$ \\
\hline$C(14)-C(1)-C(10)$ & 111.1(3) \\
\hline $\mathrm{C}(14)-\mathrm{C}(1)-\mathrm{C}(11)$ & $110.2(3)$ \\
\hline $\mathrm{O}(1)-\mathrm{C}(2)-\mathrm{C}(1)$ & $120.6(3)$ \\
\hline
\end{tabular}




\begin{tabular}{|c|c|}
\hline $\mathrm{O}(1)-\mathrm{C}(2)-\mathrm{C}(3)$ & $120.6(3)$ \\
\hline$C(3)-C(2)-C(1)$ & $118.8(3)$ \\
\hline $\mathrm{C}(2)-\mathrm{C}(3)-\mathrm{H}(3)$ & 119.1 \\
\hline $\mathrm{C}(4)-\mathrm{C}(3)-\mathrm{C}(2)$ & $121.8(3)$ \\
\hline $\mathrm{C}(4)-\mathrm{C}(3)-\mathrm{H}(3)$ & 119.1 \\
\hline $\mathrm{C}(3)-\mathrm{C}(4)-\mathrm{H}(4)$ & 118.5 \\
\hline $\mathrm{C}(3)-\mathrm{C}(4)-\mathrm{C}(5)$ & $123.1(4)$ \\
\hline $\mathrm{C}(5)-\mathrm{C}(4)-\mathrm{H}(4)$ & 118.5 \\
\hline$C(6)-C(5)-C(4)$ & $120.1(4)$ \\
\hline$C(6)-C(5)-C(10)$ & $120.0(3)$ \\
\hline$C(10)-C(5)-C(4)$ & $119.8(3)$ \\
\hline $\mathrm{C}(5)-\mathrm{C}(6)-\mathrm{H}(6)$ & 119.5 \\
\hline$C(7)-C(6)-C(5)$ & $121.0(4)$ \\
\hline $\mathrm{C}(7)-\mathrm{C}(6)-\mathrm{H}(6)$ & 119.5 \\
\hline $\mathrm{C}(6)-\mathrm{C}(7)-\mathrm{H}(7)$ & 120.7 \\
\hline$C(8)-C(7)-C(6)$ & $118.6(4)$ \\
\hline $\mathrm{C}(8)-\mathrm{C}(7)-\mathrm{H}(7)$ & 120.7 \\
\hline $\mathrm{C}(7)-\mathrm{C}(8)-\mathrm{H}(8)$ & 119.7 \\
\hline $\mathrm{C}(9)-\mathrm{C}(8)-\mathrm{C}(7)$ & $120.6(4)$ \\
\hline $\mathrm{C}(9)-\mathrm{C}(8)-\mathrm{H}(8)$ & 119.7 \\
\hline $\mathrm{C}(8)-\mathrm{C}(9)-\mathrm{H}(9)$ & 119.0 \\
\hline $\mathrm{C}(8)-\mathrm{C}(9)-\mathrm{C}(10)$ & $122.0(4)$ \\
\hline $\mathrm{C}(10)-\mathrm{C}(9)-\mathrm{H}(9)$ & 119.0 \\
\hline$C(5)-C(10)-C(1)$ & $121.5(3)$ \\
\hline$C(9)-C(10)-C(1)$ & $120.8(3)$ \\
\hline $\mathrm{C}(9)-\mathrm{C}(10)-\mathrm{C}(5)$ & $117.7(3)$ \\
\hline $\mathrm{C}(1)-\mathrm{C}(11)-\mathrm{H}(11 \mathrm{~A})$ & 108.3 \\
\hline $\mathrm{C}(1)-\mathrm{C}(11)-\mathrm{H}(11 \mathrm{~B})$ & 108.3 \\
\hline $\mathrm{H}(11 \mathrm{~A})-\mathrm{C}(11)-\mathrm{H}(11 \mathrm{~B})$ & 107.4 \\
\hline $\mathrm{C}(12)-\mathrm{C}(11)-\mathrm{C}(1)$ & $115.8(3)$ \\
\hline $\mathrm{C}(12)-\mathrm{C}(11)-\mathrm{H}(11 \mathrm{~A})$ & 108.3 \\
\hline $\mathrm{C}(12)-\mathrm{C}(11)-\mathrm{H}(11 \mathrm{~B})$ & 108.3 \\
\hline $\mathrm{N}(1)-\mathrm{C}(12)-\mathrm{C}(11)$ & $108.4(3)$ \\
\hline $\mathrm{N}(1)-\mathrm{C}(12)-\mathrm{H}(12 \mathrm{~A})$ & 110.0 \\
\hline $\mathrm{N}(1)-\mathrm{C}(12)-\mathrm{H}(12 \mathrm{~B})$ & 110.0 \\
\hline $\mathrm{C}(11)-\mathrm{C}(12)-\mathrm{H}(12 \mathrm{~A})$ & 110.0 \\
\hline $\mathrm{C}(11)-\mathrm{C}(12)-\mathrm{H}(12 \mathrm{~B})$ & 110.0 \\
\hline $\mathrm{H}(12 \mathrm{~A})-\mathrm{C}(12)-\mathrm{H}(12 \mathrm{~B})$ & 108.4 \\
\hline
\end{tabular}




\begin{tabular}{|c|c|}
\hline $\mathrm{N}(1)-\mathrm{C}(13)-\mathrm{H}(13 \mathrm{~A})$ & 109.8 \\
\hline $\mathrm{N}(1)-\mathrm{C}(13)-\mathrm{H}(13 \mathrm{~B})$ & 109.8 \\
\hline $\mathrm{N}(1)-\mathrm{C}(13)-\mathrm{C}(14)$ & $109.3(3)$ \\
\hline $\mathrm{H}(13 \mathrm{~A})-\mathrm{C}(13)-\mathrm{H}(13 \mathrm{~B})$ & 108.3 \\
\hline $\mathrm{C}(14)-\mathrm{C}(13)-\mathrm{H}(13 \mathrm{~A})$ & 109.8 \\
\hline $\mathrm{C}(14)-\mathrm{C}(13)-\mathrm{H}(13 \mathrm{~B})$ & 109.8 \\
\hline $\mathrm{C}(13)-\mathrm{C}(14)-\mathrm{C}(1)$ & 116.1(3) \\
\hline $\mathrm{C}(15)-\mathrm{C}(14)-\mathrm{C}(1)$ & $124.2(3)$ \\
\hline $\mathrm{C}(15)-\mathrm{C}(14)-\mathrm{C}(13)$ & $119.7(3)$ \\
\hline $\mathrm{C}(14)-\mathrm{C}(15)-\mathrm{H}(15 \mathrm{~A})$ & 120.0 \\
\hline $\mathrm{C}(14)-\mathrm{C}(15)-\mathrm{H}(15 \mathrm{~B})$ & 120.0 \\
\hline $\mathrm{H}(15 \mathrm{~A})-\mathrm{C}(15)-\mathrm{H}(15 \mathrm{~B})$ & 120.0 \\
\hline$C(17)-C(16)-S(1)$ & $120.9(3)$ \\
\hline $\mathrm{C}(21)-\mathrm{C}(16)-\mathrm{S}(1)$ & $120.0(3)$ \\
\hline $\mathrm{C}(21)-\mathrm{C}(16)-\mathrm{C}(17)$ & $119.1(3)$ \\
\hline $\mathrm{C}(16)-\mathrm{C}(17)-\mathrm{H}(17)$ & 119.8 \\
\hline $\mathrm{C}(18)-\mathrm{C}(17)-\mathrm{C}(16)$ & $120.4(3)$ \\
\hline $\mathrm{C}(18)-\mathrm{C}(17)-\mathrm{H}(17)$ & 119.8 \\
\hline $\mathrm{C}(17)-\mathrm{C}(18)-\mathrm{H}(18)$ & 119.2 \\
\hline $\mathrm{C}(17)-\mathrm{C}(18)-\mathrm{C}(19)$ & $121.7(3)$ \\
\hline $\mathrm{C}(19)-\mathrm{C}(18)-\mathrm{H}(18)$ & 119.2 \\
\hline $\mathrm{C}(18)-\mathrm{C}(19)-\mathrm{C}(20)$ & $118.4(3)$ \\
\hline$C(18)-C(19)-C(22)$ & $122.3(4)$ \\
\hline $\mathrm{C}(20)-\mathrm{C}(19)-\mathrm{C}(22)$ & 119.3(4) \\
\hline $\mathrm{C}(19)-\mathrm{C}(20)-\mathrm{H}(20)$ & 119.9 \\
\hline$C(19)-C(20)-C(21)$ & $120.2(3)$ \\
\hline $\mathrm{C}(21)-\mathrm{C}(20)-\mathrm{H}(20)$ & 119.9 \\
\hline$C(16)-C(21)-C(20)$ & $120.2(3)$ \\
\hline $\mathrm{C}(16)-\mathrm{C}(21)-\mathrm{H}(21)$ & 119.9 \\
\hline $\mathrm{C}(20)-\mathrm{C}(21)-\mathrm{H}(21)$ & 119.9 \\
\hline $\mathrm{C}(19)-\mathrm{C}(22)-\mathrm{H}(22 \mathrm{~A})$ & 109.5 \\
\hline $\mathrm{C}(19)-\mathrm{C}(22)-\mathrm{H}(22 \mathrm{~B})$ & 109.5 \\
\hline $\mathrm{C}(19)-\mathrm{C}(22)-\mathrm{H}(22 \mathrm{C})$ & 109.5 \\
\hline $\mathrm{H}(22 \mathrm{~A})-\mathrm{C}(22)-\mathrm{H}(22 \mathrm{~B})$ & 109.5 \\
\hline $\mathrm{H}(22 \mathrm{~A})-\mathrm{C}(22)-\mathrm{H}(22 \mathrm{C})$ & 109.5 \\
\hline $\mathrm{H}(22 \mathrm{~B})-\mathrm{C}(22)-\mathrm{H}(22 \mathrm{C})$ & 109.5 \\
\hline
\end{tabular}

Symmetry transformations used to generate equivalent atoms: 
Table S4. Anisotropic displacement parameters $\left(\AA^{2} \times 10^{3}\right)$ for mj118252_0m. The anisotropic displacement factor exponent takes the form: $\quad-2 \pi^{2}\left[h^{2} a^{* 2} U^{11}+\ldots+2 h k a^{*} b^{*} U^{12}\right]$

\begin{tabular}{|c|c|c|c|c|c|c|}
\hline & $\mathrm{U}^{11}$ & $\mathrm{U}^{22}$ & $\mathrm{U}^{33}$ & $\mathrm{U}^{23}$ & $\mathrm{U}^{13}$ & $\mathrm{U}^{12}$ \\
\hline$S(1)$ & $44(1)$ & $58(1)$ & $72(1)$ & $-7(1)$ & $1(1)$ & $-3(1)$ \\
\hline $\mathrm{O}(1)$ & $49(2)$ & $62(2)$ & $98(2)$ & $-10(1)$ & $1(1)$ & $13(1)$ \\
\hline $\mathrm{O}(2)$ & $56(2)$ & $72(2)$ & $78(2)$ & $-2(2)$ & $-4(1)$ & $7(1)$ \\
\hline $\mathrm{O}(3)$ & $44(1)$ & $70(2)$ & $83(2)$ & $-16(1)$ & $4(1)$ & $-17(1)$ \\
\hline $\mathrm{N}(1)$ & $47(2)$ & $47(2)$ & $65(2)$ & $-1(1)$ & $0(1)$ & $-8(1)$ \\
\hline $\mathrm{C}(1)$ & $41(2)$ & $46(2)$ & $68(2)$ & $0(2)$ & $-3(2)$ & $-1(2)$ \\
\hline $\mathrm{C}(2)$ & $47(2)$ & $36(2)$ & $80(3)$ & $3(2)$ & $-1(2)$ & $-1(2)$ \\
\hline $\mathrm{C}(3)$ & $47(2)$ & $56(2)$ & $85(3)$ & $7(2)$ & $-11(2)$ & $-5(2)$ \\
\hline $\mathrm{C}(4)$ & $58(2)$ & $58(2)$ & $75(3)$ & $-4(2)$ & $-7(2)$ & $-9(2)$ \\
\hline$C(5)$ & $52(2)$ & $40(2)$ & $73(3)$ & $0(2)$ & $0(2)$ & $-2(2)$ \\
\hline $\mathrm{C}(6)$ & $76(3)$ & $47(2)$ & $72(3)$ & $-1(2)$ & $4(2)$ & $-5(2)$ \\
\hline $\mathrm{C}(7)$ & $76(3)$ & $45(2)$ & $83(3)$ & $1(2)$ & $15(2)$ & $7(2)$ \\
\hline $\mathrm{C}(8)$ & $57(2)$ & $56(2)$ & $87(3)$ & $8(2)$ & $12(2)$ & $8(2)$ \\
\hline $\mathrm{C}(9)$ & $53(2)$ & $53(2)$ & $77(3)$ & $-2(2)$ & $3(2)$ & $6(2)$ \\
\hline$C(10)$ & $46(2)$ & $37(2)$ & $72(2)$ & $3(2)$ & $1(2)$ & $-1(2)$ \\
\hline $\mathrm{C}(11)$ & $47(2)$ & $40(2)$ & $78(3)$ & $-1(2)$ & $2(2)$ & $-3(2)$ \\
\hline $\mathrm{C}(12)$ & $42(2)$ & $46(2)$ & $74(2)$ & $2(2)$ & $4(2)$ & $-5(2)$ \\
\hline $\mathrm{C}(13)$ & $60(2)$ & $42(2)$ & $76(3)$ & $-4(2)$ & $10(2)$ & $-7(2)$ \\
\hline $\mathrm{C}(14)$ & $48(2)$ & $38(2)$ & $66(2)$ & $-1(2)$ & $-2(2)$ & $-3(2)$ \\
\hline$C(15)$ & $71(3)$ & $56(2)$ & $81(3)$ & $0(2)$ & $9(2)$ & $-12(2)$ \\
\hline$C(16)$ & $50(2)$ & $50(2)$ & $60(2)$ & 2(2) & $-3(2)$ & $-9(2)$ \\
\hline $\mathrm{C}(17)$ & $55(2)$ & $52(2)$ & 79(3) & $6(2)$ & $8(2)$ & $-11(2)$ \\
\hline $\mathrm{C}(18)$ & $63(2)$ & $45(2)$ & $82(3)$ & $8(2)$ & $6(2)$ & $7(2)$ \\
\hline$C(19)$ & $56(2)$ & $57(2)$ & $66(2)$ & $7(2)$ & $1(2)$ & $10(2)$ \\
\hline $\mathrm{C}(20)$ & $48(2)$ & $52(2)$ & $72(2)$ & $1(2)$ & $7(2)$ & $0(2)$ \\
\hline $\mathrm{C}(21)$ & $54(2)$ & $42(2)$ & $79(3)$ & $0(2)$ & $2(2)$ & $0(2)$ \\
\hline$C(22)$ & $74(3)$ & $85(3)$ & $94(3)$ & $16(3)$ & $10(2)$ & $32(2)$ \\
\hline
\end{tabular}


Table S5. Hydrogen coordinates ( x 10 $)$ and isotropic displacement parameters $\left(\AA^{2} \times 10^{3}\right)$ for mj118252_0m.

\begin{tabular}{|c|c|c|c|c|}
\hline & $\mathrm{x}$ & $\mathrm{y}$ & z & $\mathrm{U}(\mathrm{eq})$ \\
\hline $\mathrm{H}(3)$ & 4864 & 4024 & 6241 & 75 \\
\hline $\mathrm{H}(4)$ & 3953 & 5177 & 6765 & 76 \\
\hline $\mathrm{H}(6)$ & 2486 & 6394 & 6897 & 78 \\
\hline $\mathrm{H}(7)$ & 876 & 7169 & 6590 & 82 \\
\hline $\mathrm{H}(8)$ & 103 & 6659 & 5715 & 80 \\
\hline $\mathrm{H}(9)$ & 975 & 5497 & 5133 & 73 \\
\hline $\mathrm{H}(11 \mathrm{~A})$ & 2376 & 5433 & 4567 & 66 \\
\hline $\mathrm{H}(11 \mathrm{~B})$ & 3645 & 5509 & 4702 & 66 \\
\hline $\mathrm{H}(12 \mathrm{~A})$ & 3283 & 4731 & 3792 & 65 \\
\hline $\mathrm{H}(12 \mathrm{~B})$ & 3988 & 4004 & 4205 & 65 \\
\hline $\mathrm{H}(13 \mathrm{~A})$ & 3151 & 2570 & 4682 & 71 \\
\hline $\mathrm{H}(13 \mathrm{~B})$ & 1881 & 2339 & 4595 & 71 \\
\hline $\mathrm{H}(15 \mathrm{~A})$ & 1050 & 2577 & 5470 & 83 \\
\hline $\mathrm{H}(15 \mathrm{~B})$ & 1254 & 3595 & 5870 & 83 \\
\hline $\mathrm{H}(17)$ & 3115 & 1037 & 3609 & 74 \\
\hline $\mathrm{H}(18)$ & 4686 & 277 & 3291 & 76 \\
\hline $\mathrm{H}(20)$ & 5670 & 2926 & 2592 & 69 \\
\hline $\mathrm{H}(21)$ & 4054 & 3695 & 2899 & 70 \\
\hline $\mathrm{H}(22 \mathrm{~A})$ & 6398 & 1040 & 2283 & 127 \\
\hline $\mathrm{H}(22 \mathrm{~B})$ & 6418 & 329 & 2844 & 127 \\
\hline $\mathrm{H}(22 \mathrm{C})$ & 6998 & 1422 & 2856 & 127 \\
\hline
\end{tabular}




\section{Copies of NMR spectra}

Figure $1{ }^{1} \mathrm{H}$ NMR spectrum of $\mathbf{1 a}\left(400 \mathrm{MHz}, \mathrm{CDCl}_{3}\right)$

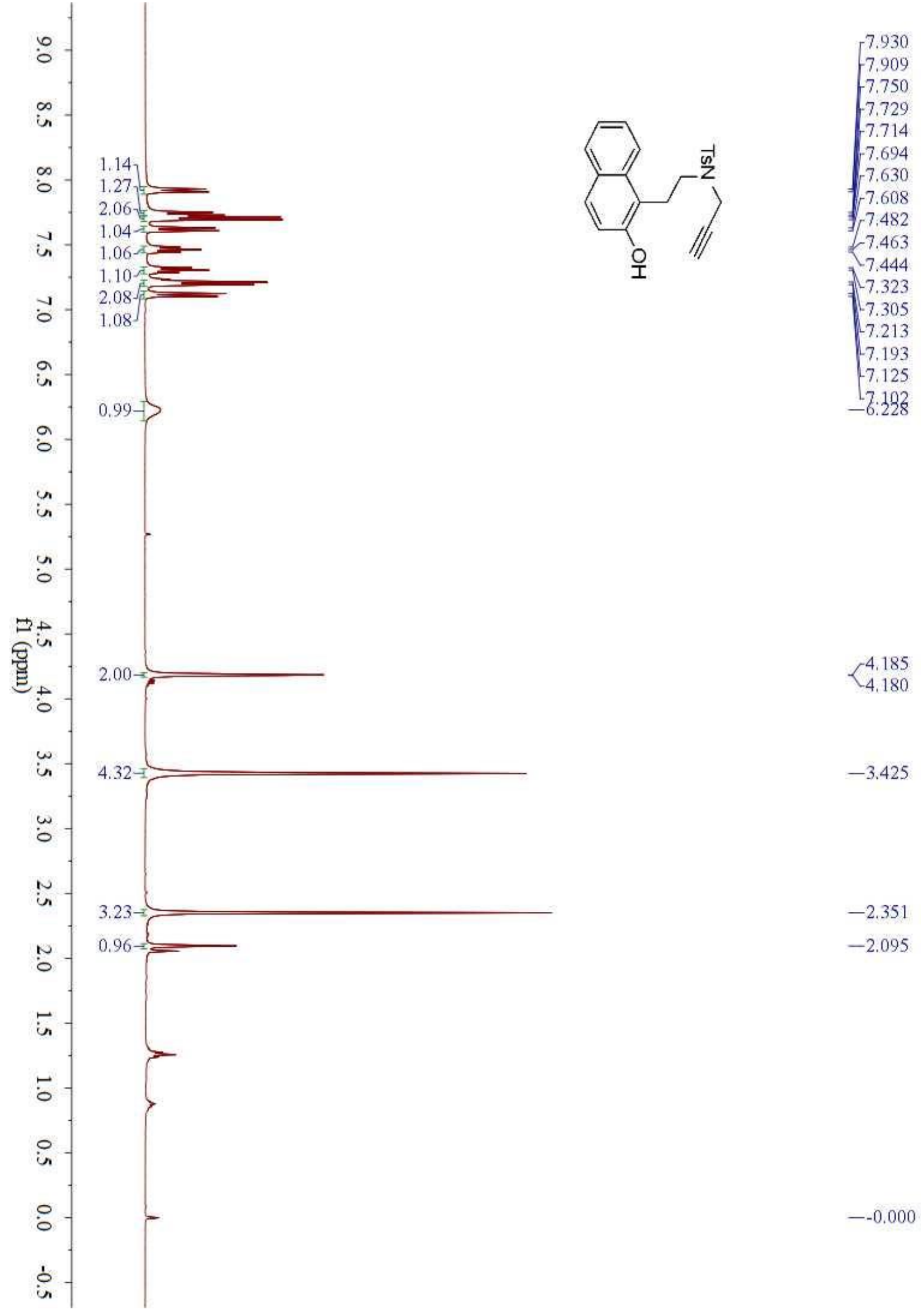


Figure $2{ }^{13} \mathrm{C}$ NMR spectrum of $\mathbf{1 a}\left(100 \mathrm{MHz}, \mathrm{CDCl}_{3}\right)$

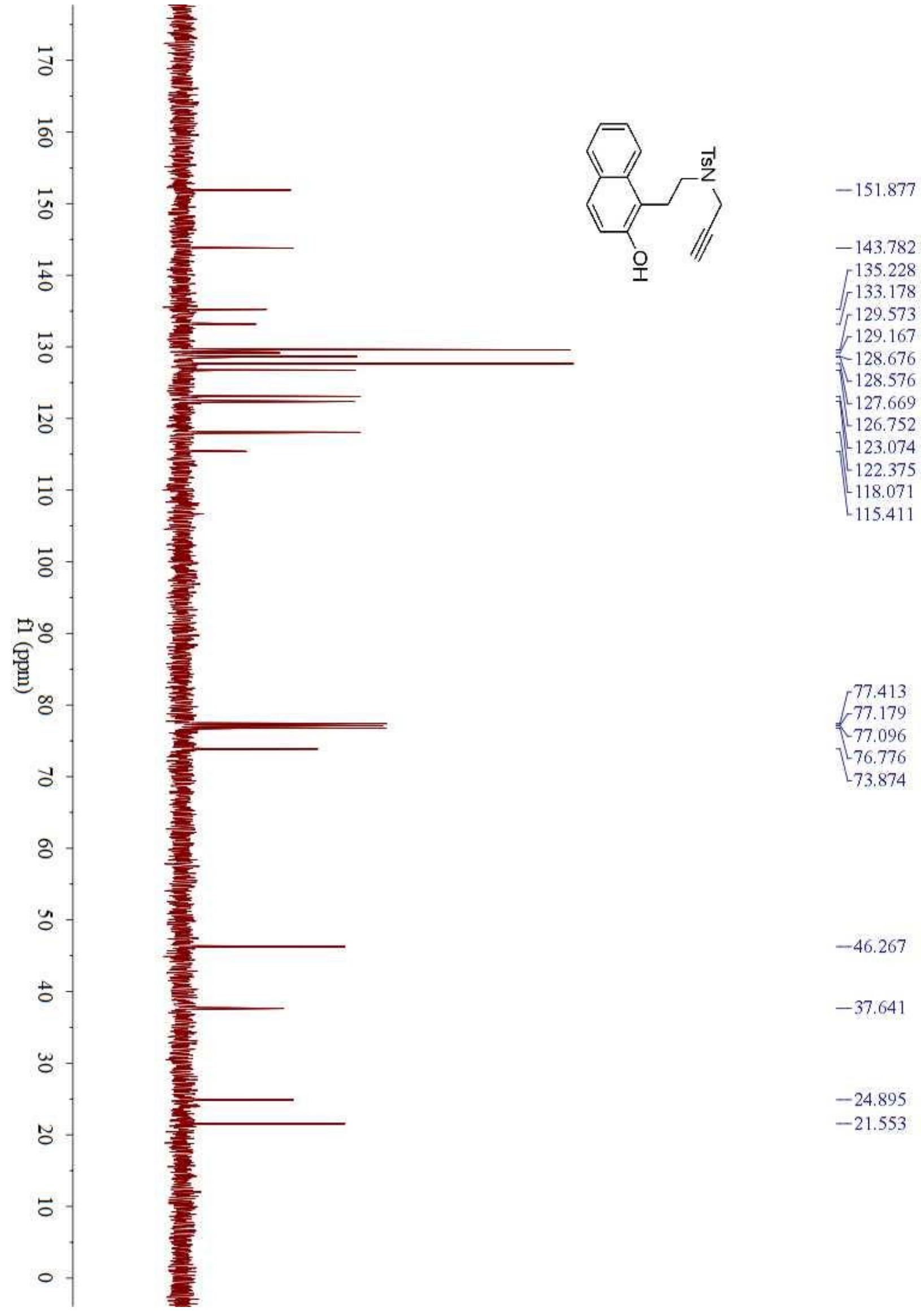


Figure $3{ }^{1} \mathrm{H}$ NMR spectrum of $\mathbf{1 b}\left(400 \mathrm{MHz}, \mathrm{CDCl}_{3}\right)$

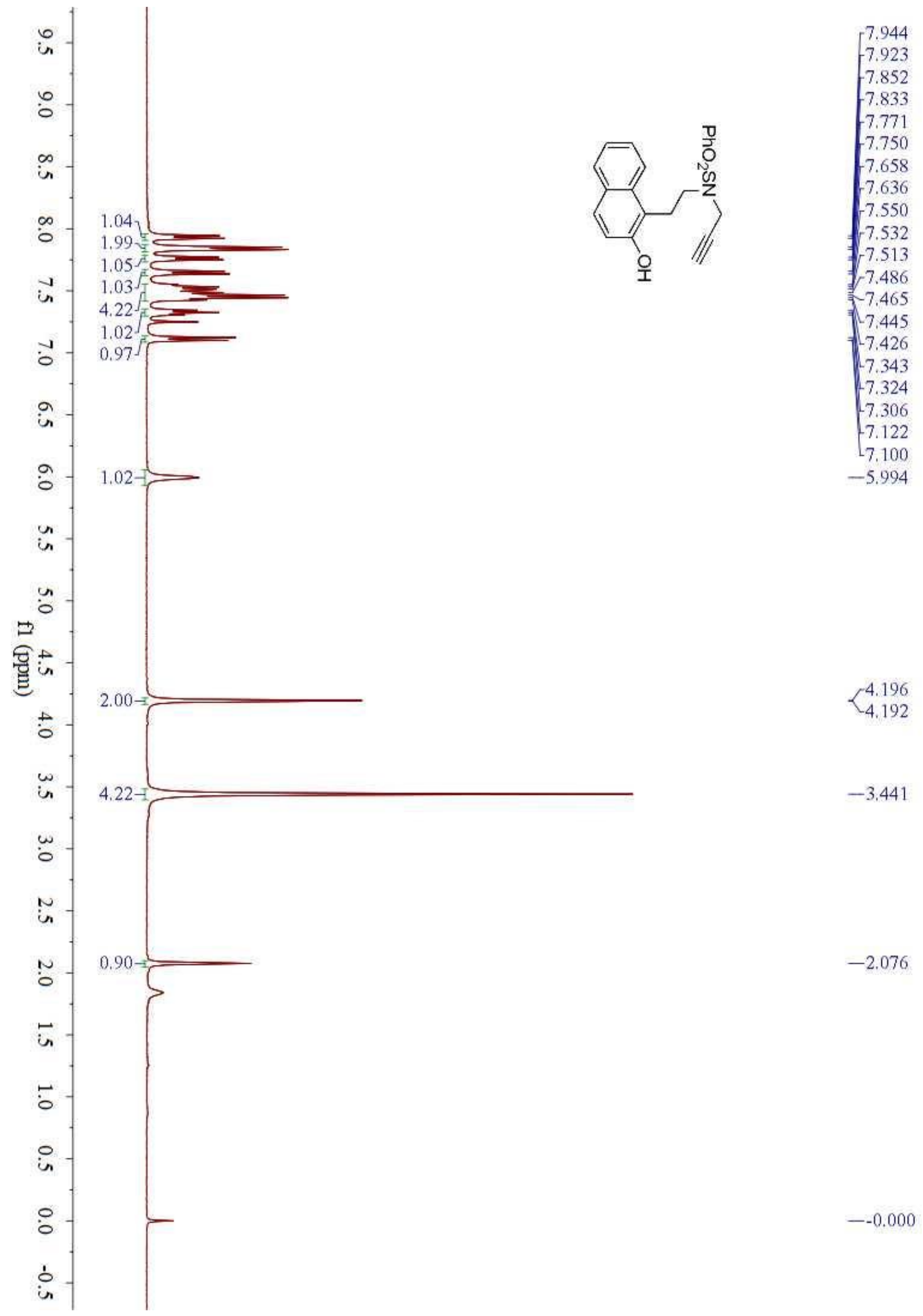


Figure $4{ }^{13} \mathrm{C}$ NMR spectrum of $\mathbf{1 b}\left(100 \mathrm{MHz}, \mathrm{CDCl}_{3}\right)$

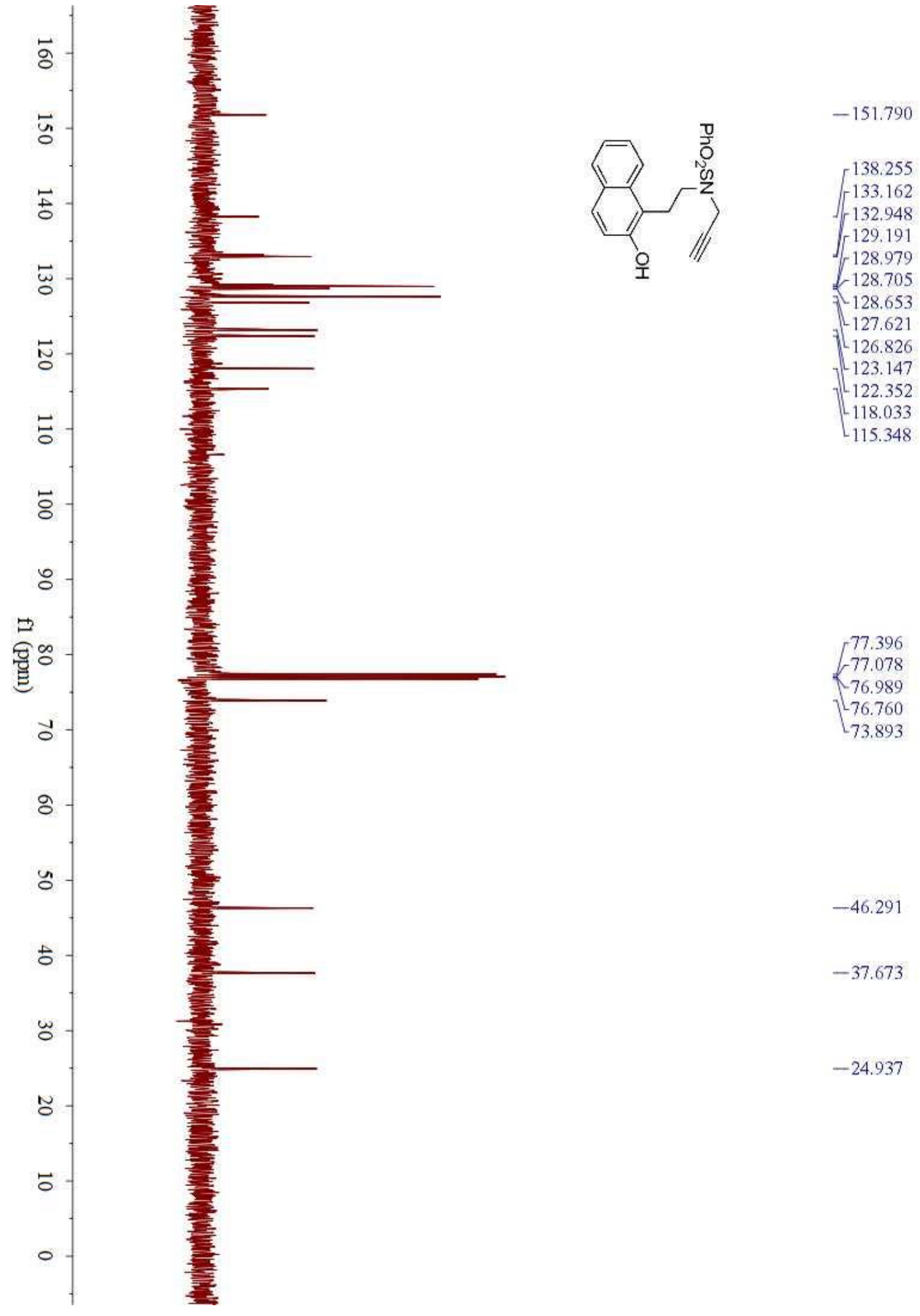


Figure $5{ }^{1} \mathrm{H}$ NMR spectrum of $1 \mathbf{c}\left(400 \mathrm{MHz}, \mathrm{CDCl}_{3}\right)$

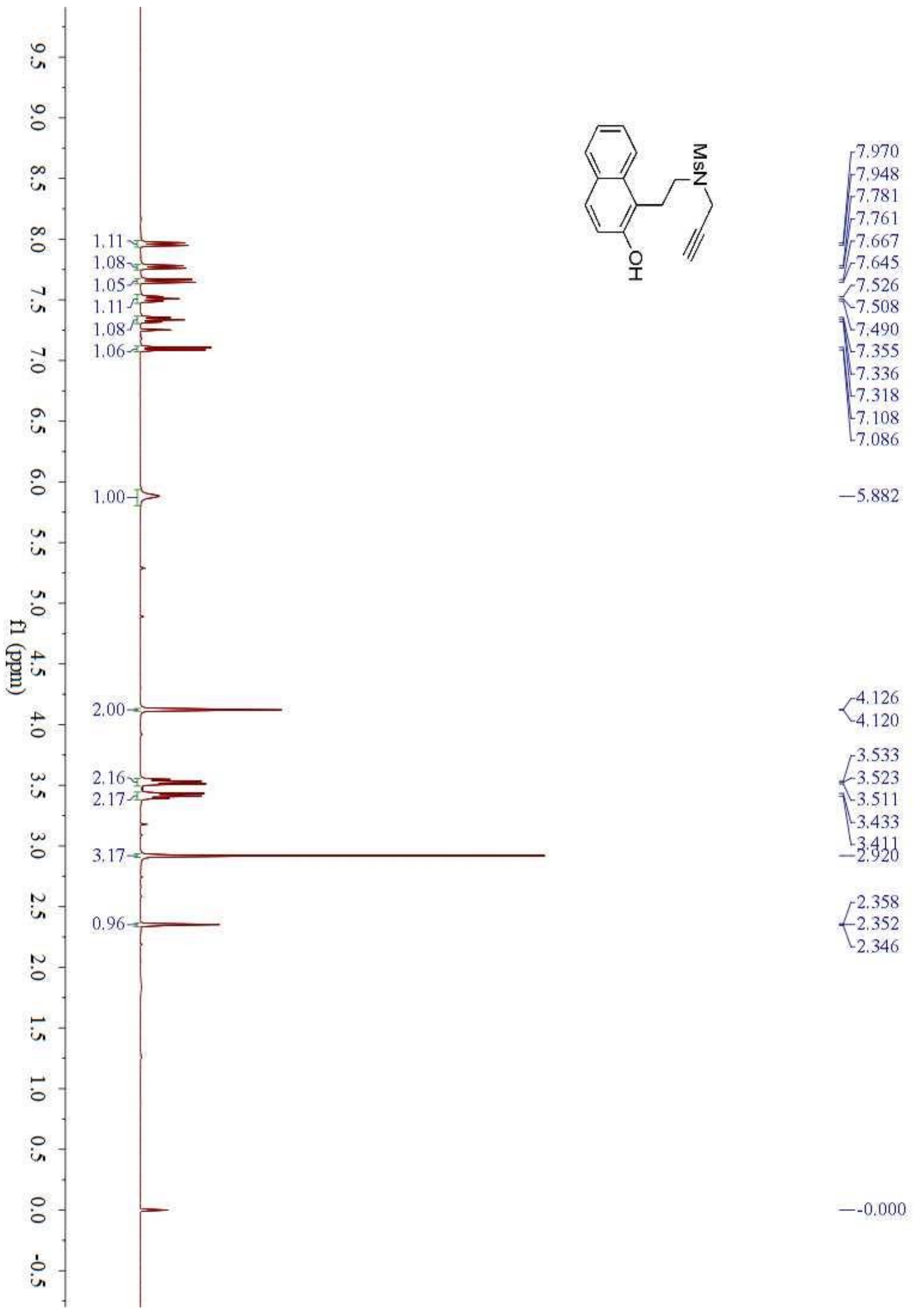


Figure $6{ }^{13} \mathrm{C} \mathrm{NMR}$ spectrum of $1 \mathrm{c}\left(100 \mathrm{MHz}, \mathrm{CDCl}_{3}\right)$

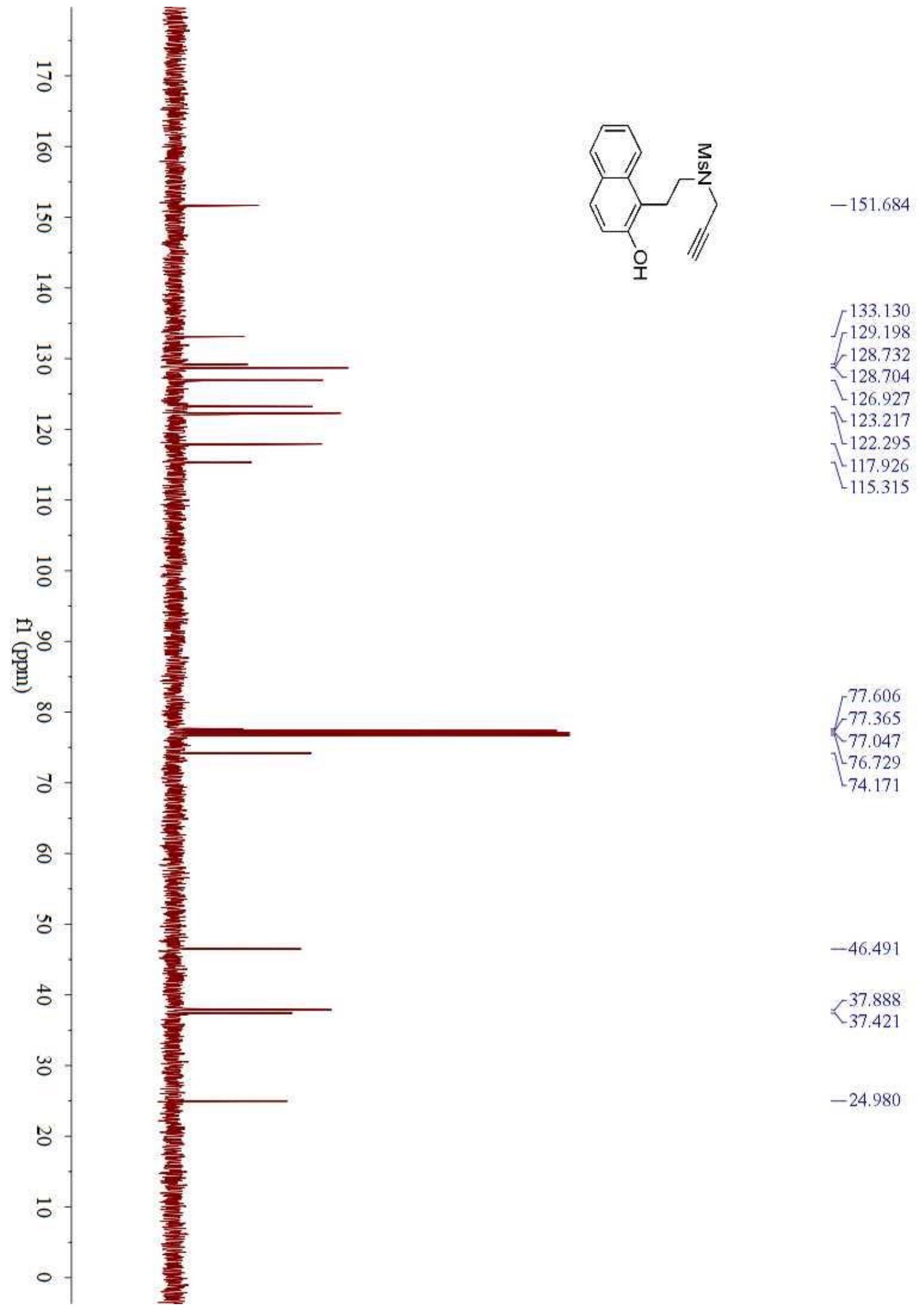


Figure $7{ }^{1} \mathrm{H}$ NMR spectrum of $\mathbf{1 d}\left(400 \mathrm{MHz}, \mathrm{CDCl}_{3}\right)$

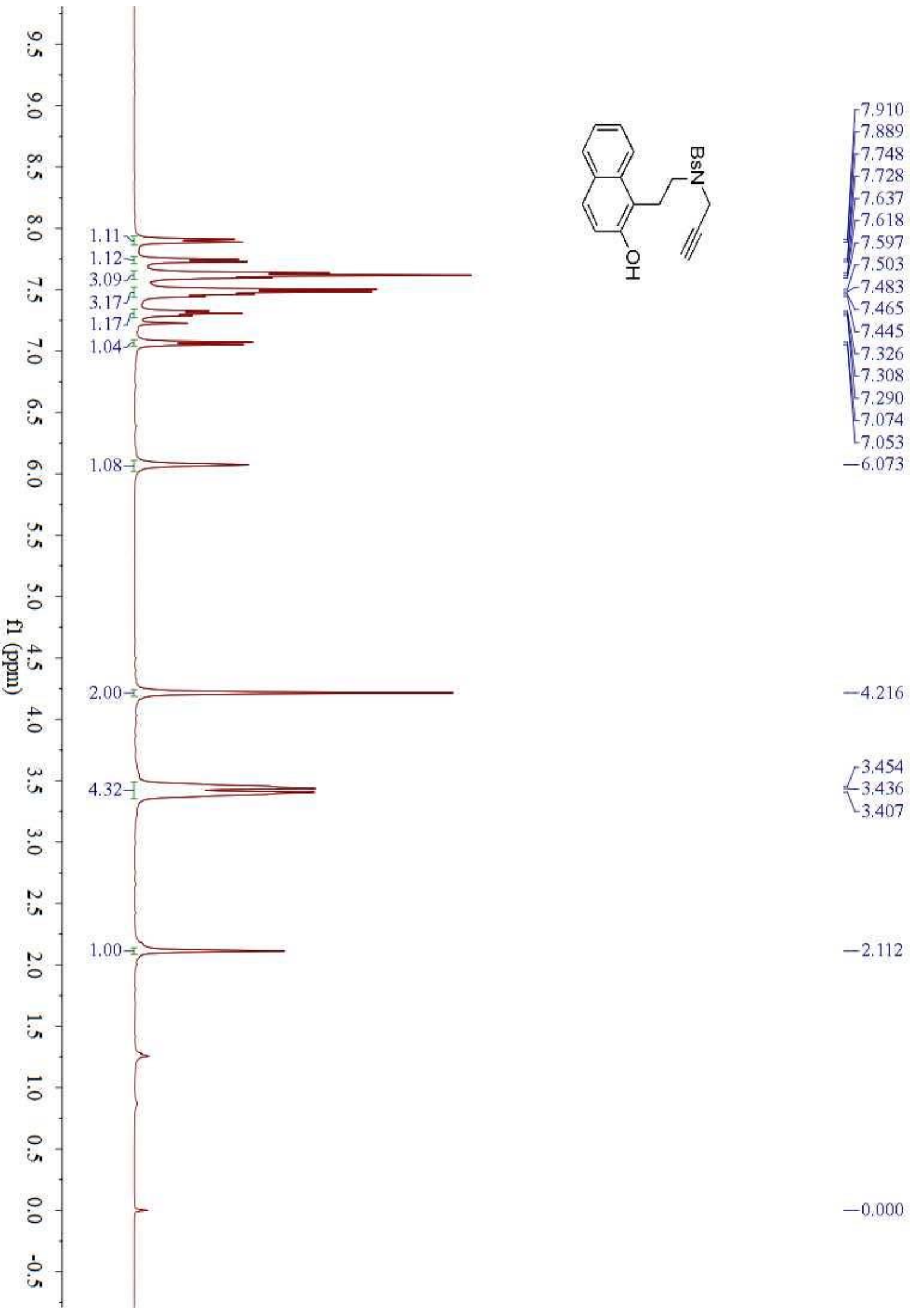


Figure $8^{13} \mathrm{C}$ NMR spectrum of $\mathbf{1 d}\left(100 \mathrm{MHz}, \mathrm{CDCl}_{3}\right)$
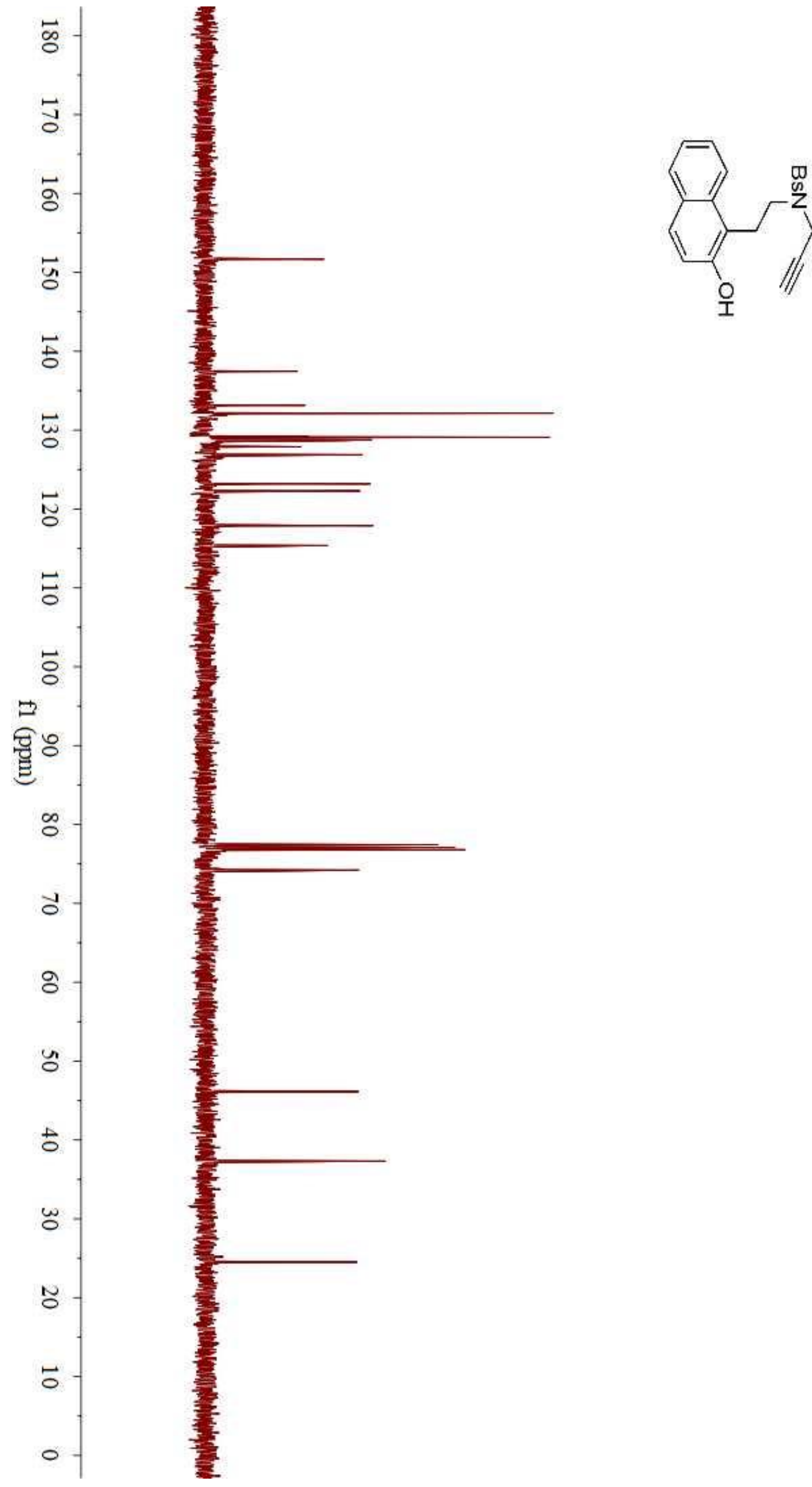

$-151.679$

$-137.455$

$-133.153$

$-132.141$

129.196

129.100

$-128.768$

128.666

127.894

$-126.876$

123.195

$-122.282$

$-117.886$

$-115.379$

77.424

$-77.105$

$-76.842$

$-76.787$

$-74.213$

$-46.139$

$-37.322$

$-24.530$ 
Figure $9{ }^{1} \mathrm{H}$ NMR spectrum of $1 \mathbf{e}\left(400 \mathrm{MHz}, \mathrm{CDCl}_{3}\right)$

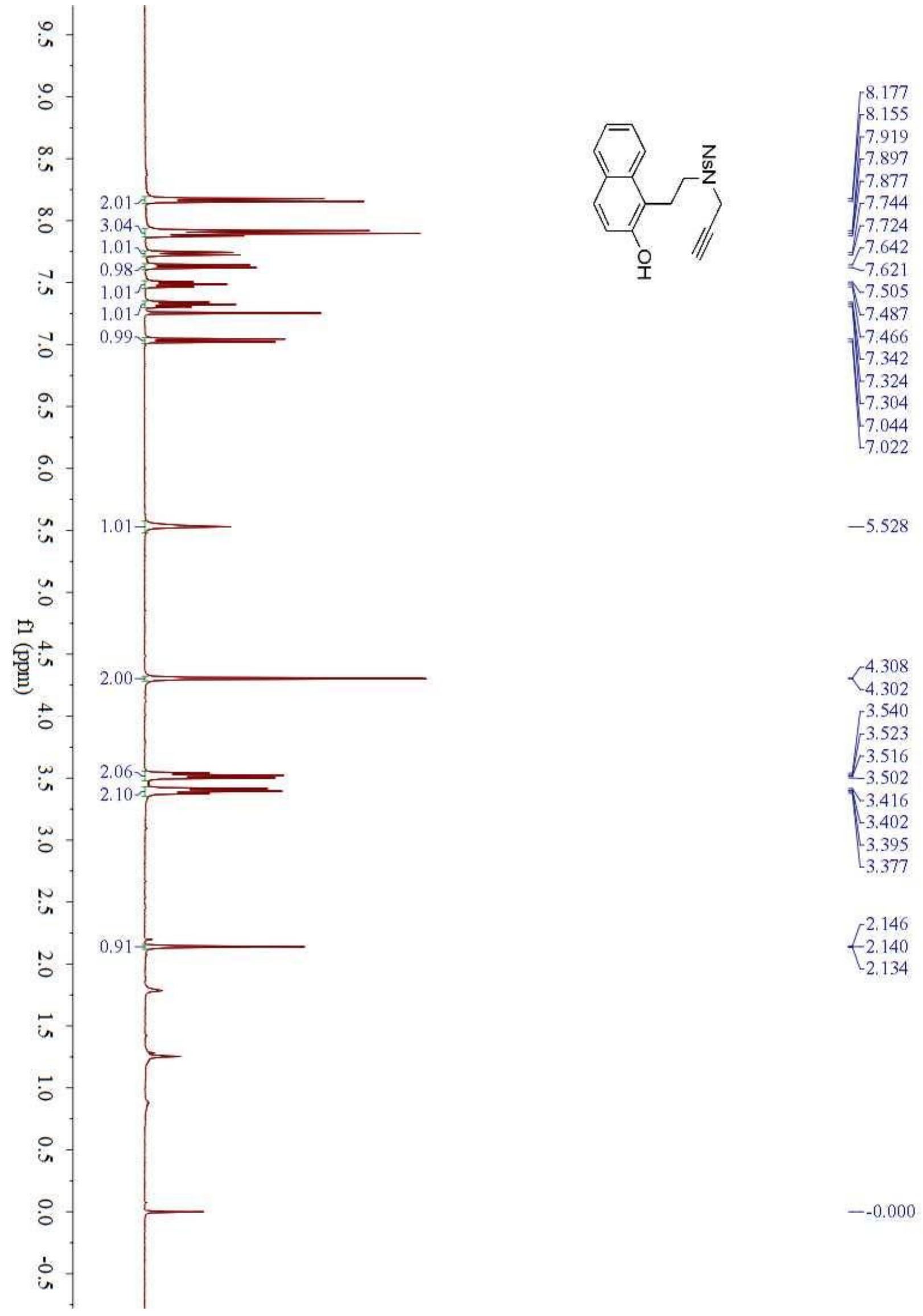


Figure $10{ }^{13} \mathrm{C}$ NMR spectrum of 1 e $\left(100 \mathrm{MHz}, \mathrm{CDCl}_{3}\right)$
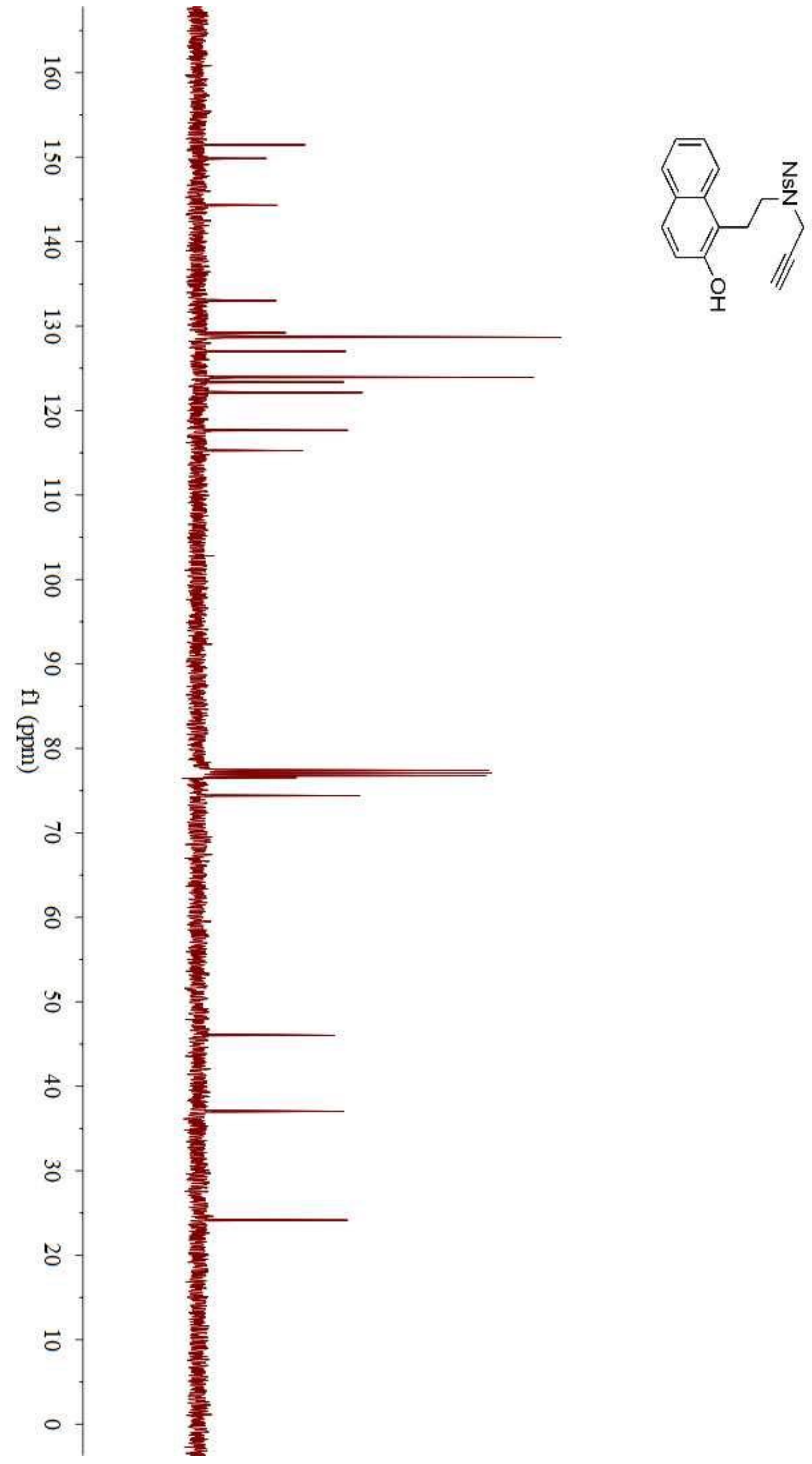

$-151.462$

$-149.848$

$-144.308$

133.032

129.210

128.754

$L_{128.675}$

$-127.001$

- 123.926

123.354

$-122.144$

ᄂ 117.659

$-115.250$

77.397

$-77.078$

$-76.761$

$-76.568$

$-74.401$ 
Figure $11{ }^{1} \mathrm{H}$ NMR spectrum of $1 \mathbf{f}\left(400 \mathrm{MHz}, \mathrm{d}_{6}\right.$ - Acetone)

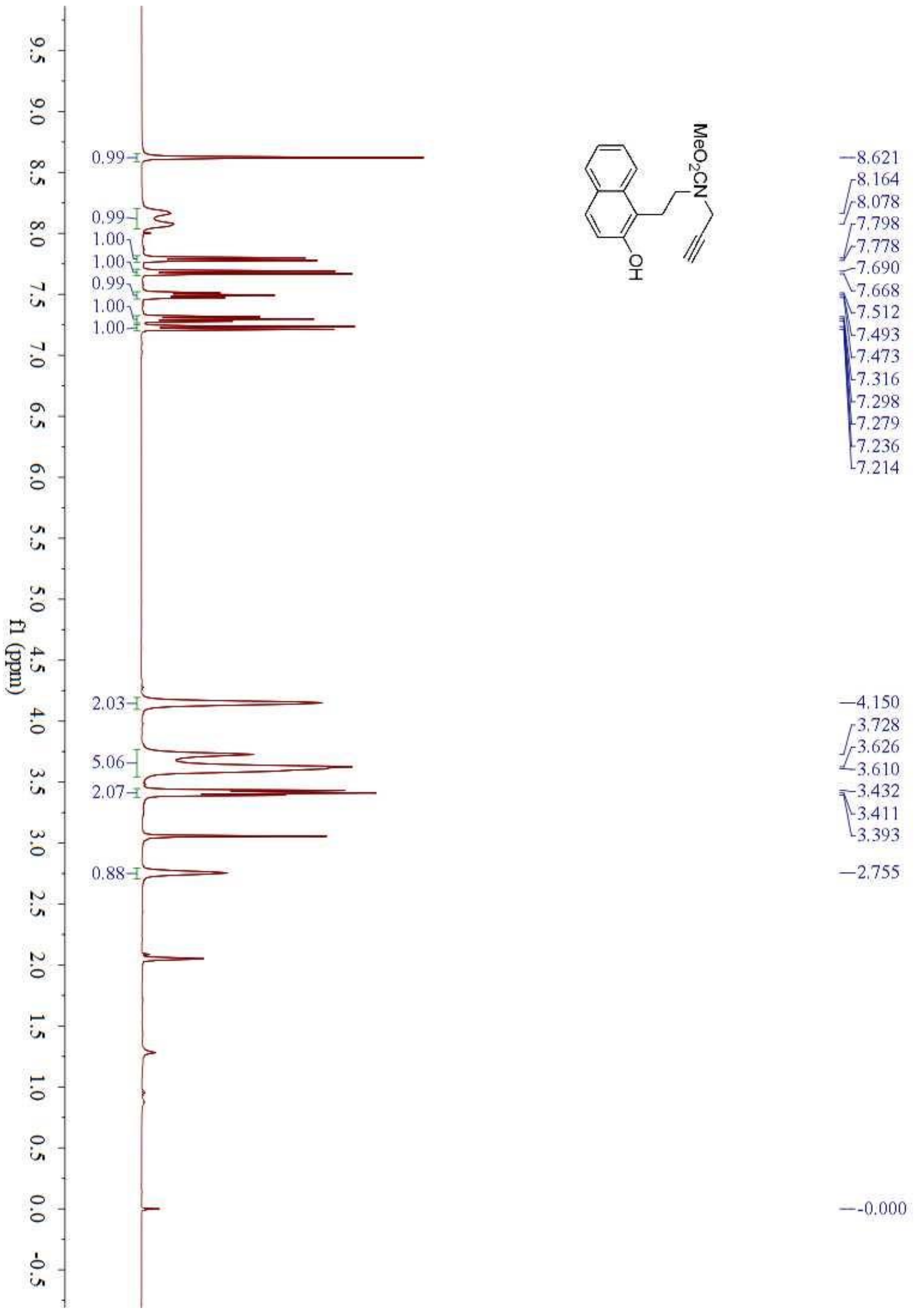


Figure $12{ }^{13} \mathrm{C}$ NMR spectrum of $\mathbf{1 f}\left(100 \mathrm{MHz}, \mathrm{d}_{6}\right.$ - Acetone)

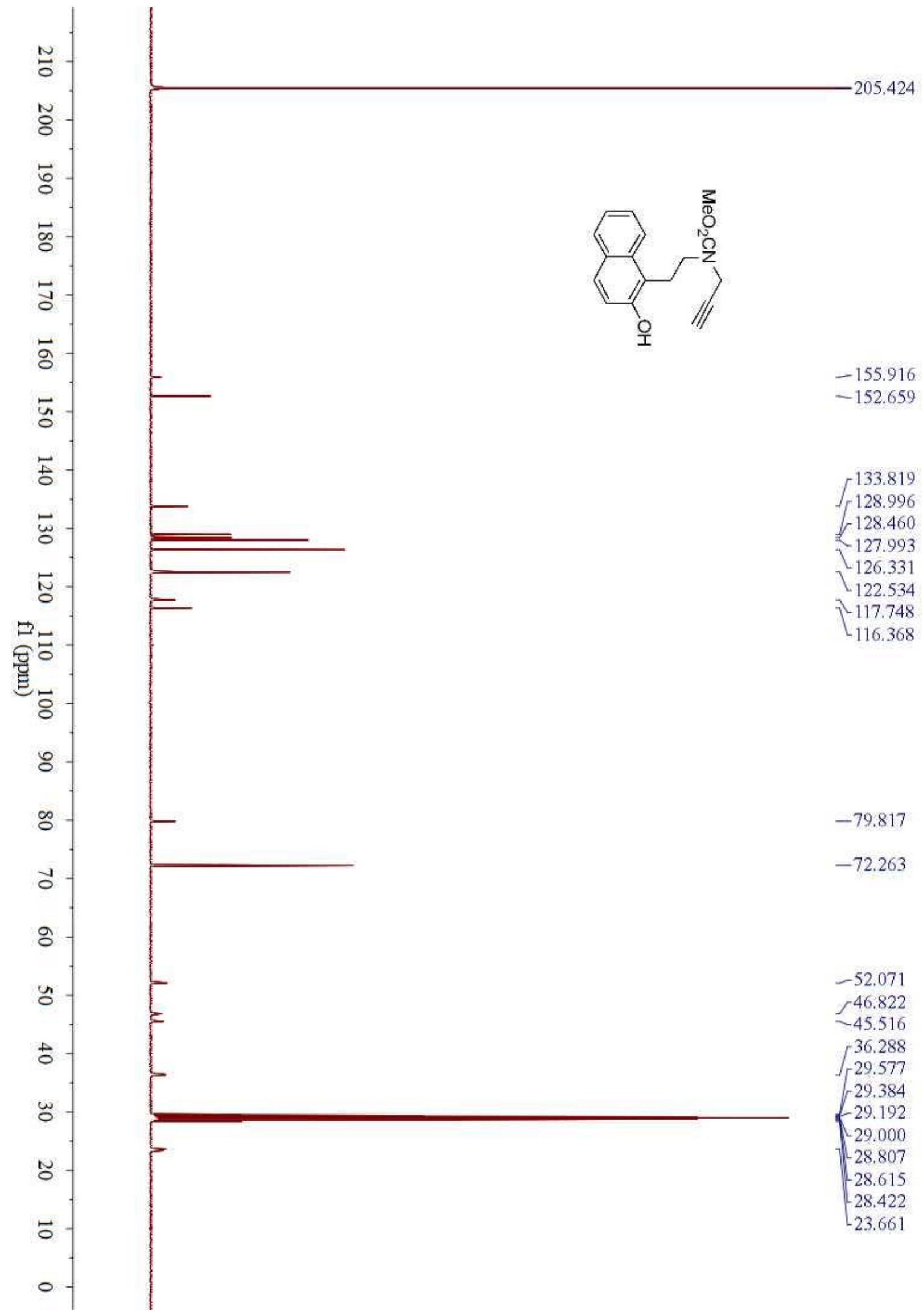


Figure $13{ }^{1} \mathrm{H}$ NMR spectrum of 1 g $\left(400 \mathrm{MHz}, \mathrm{CDCl}_{3}\right)$

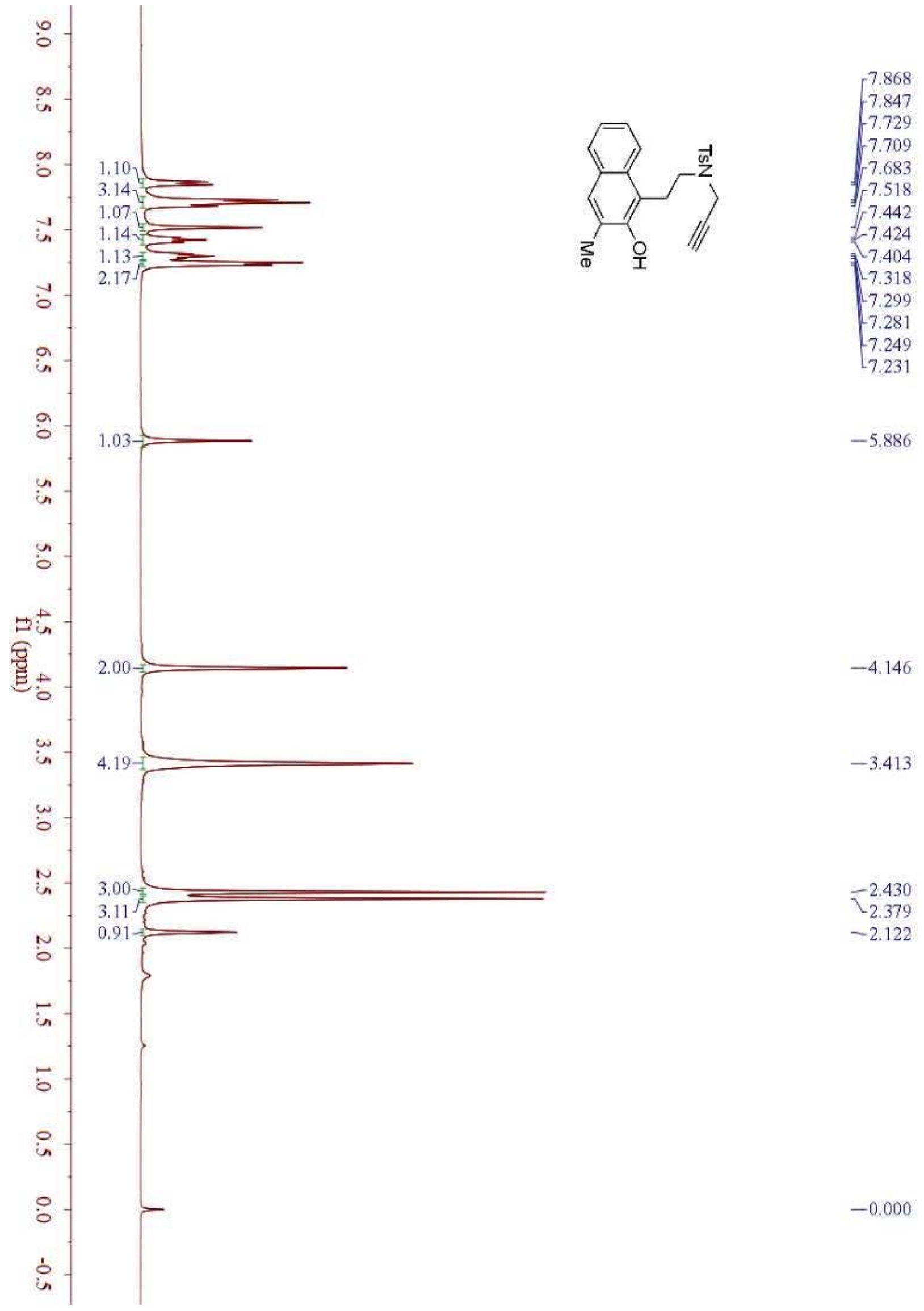


Figure $14{ }^{13} \mathrm{C} \mathrm{NMR}$ spectrum of $1 \mathrm{~g}\left(100 \mathrm{MHz}, \mathrm{CDCl}_{3}\right)$

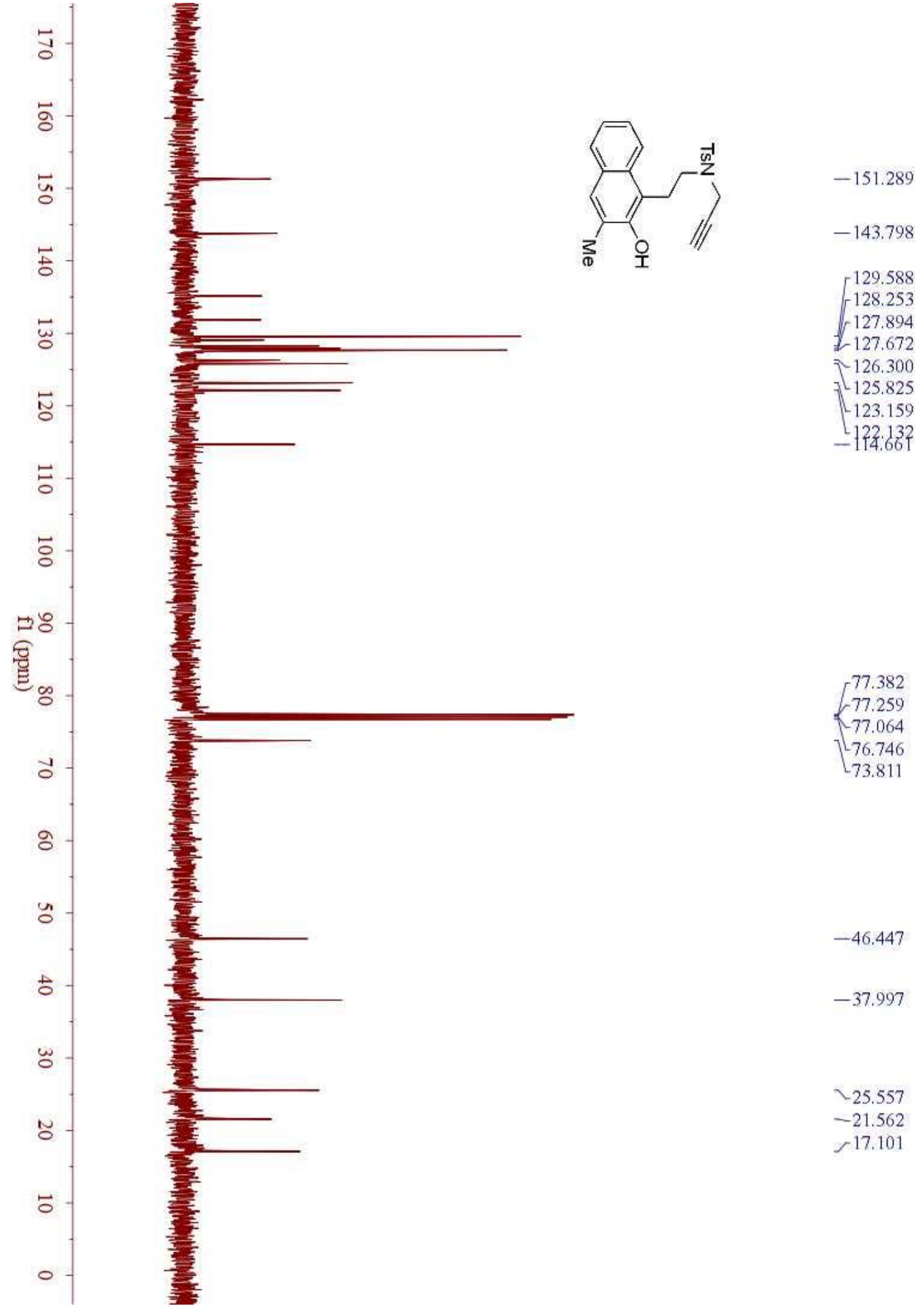


Figure $15{ }^{1} \mathrm{H}$ NMR spectrum of $\mathbf{1 h}\left(400 \mathrm{MHz}, \mathrm{CDCl}_{3}\right)$

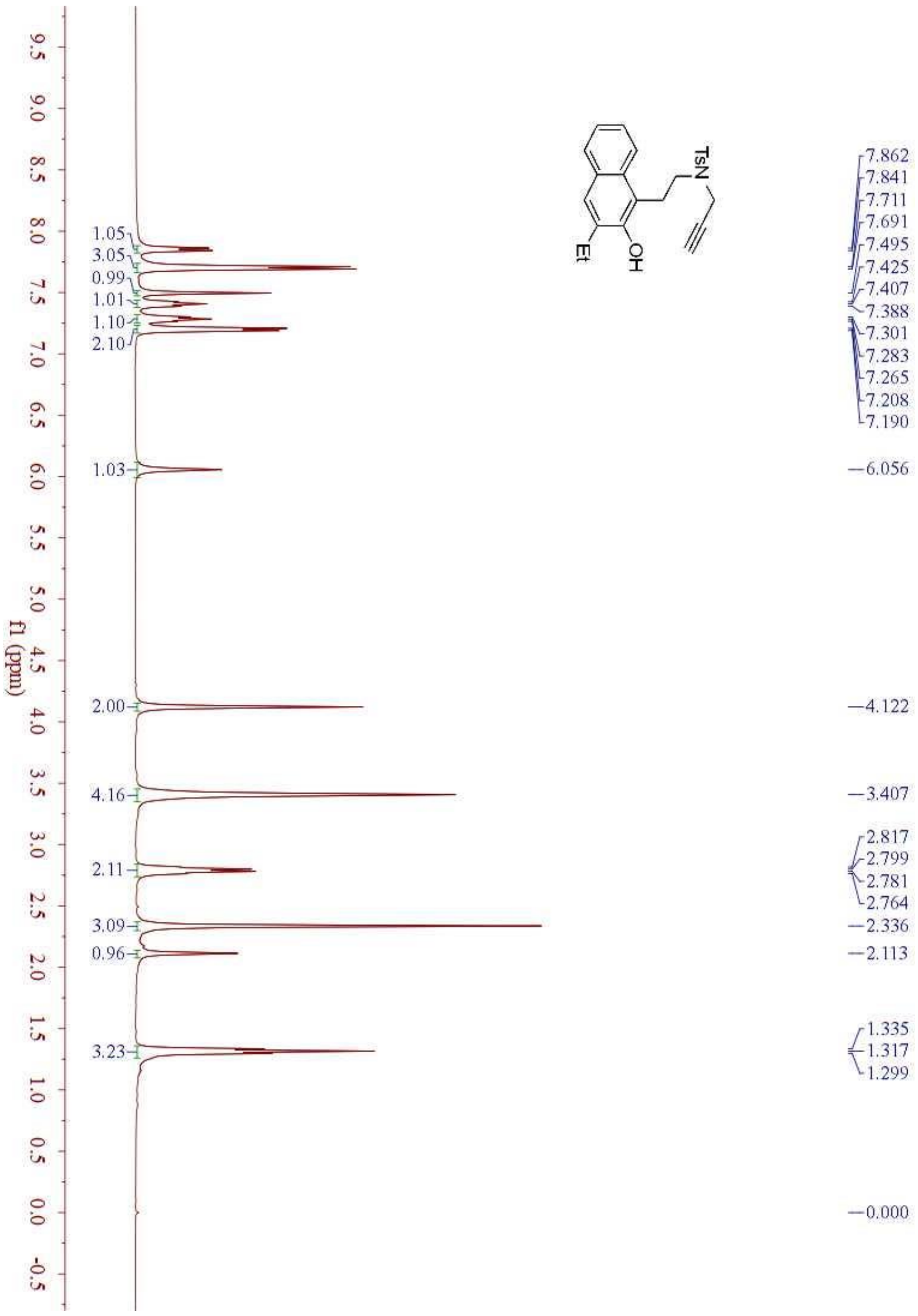


Figure $16{ }^{13} \mathrm{C}$ NMR spectrum of $\mathbf{1 h}\left(100 \mathrm{MHz}, \mathrm{CDCl}_{3}\right)$

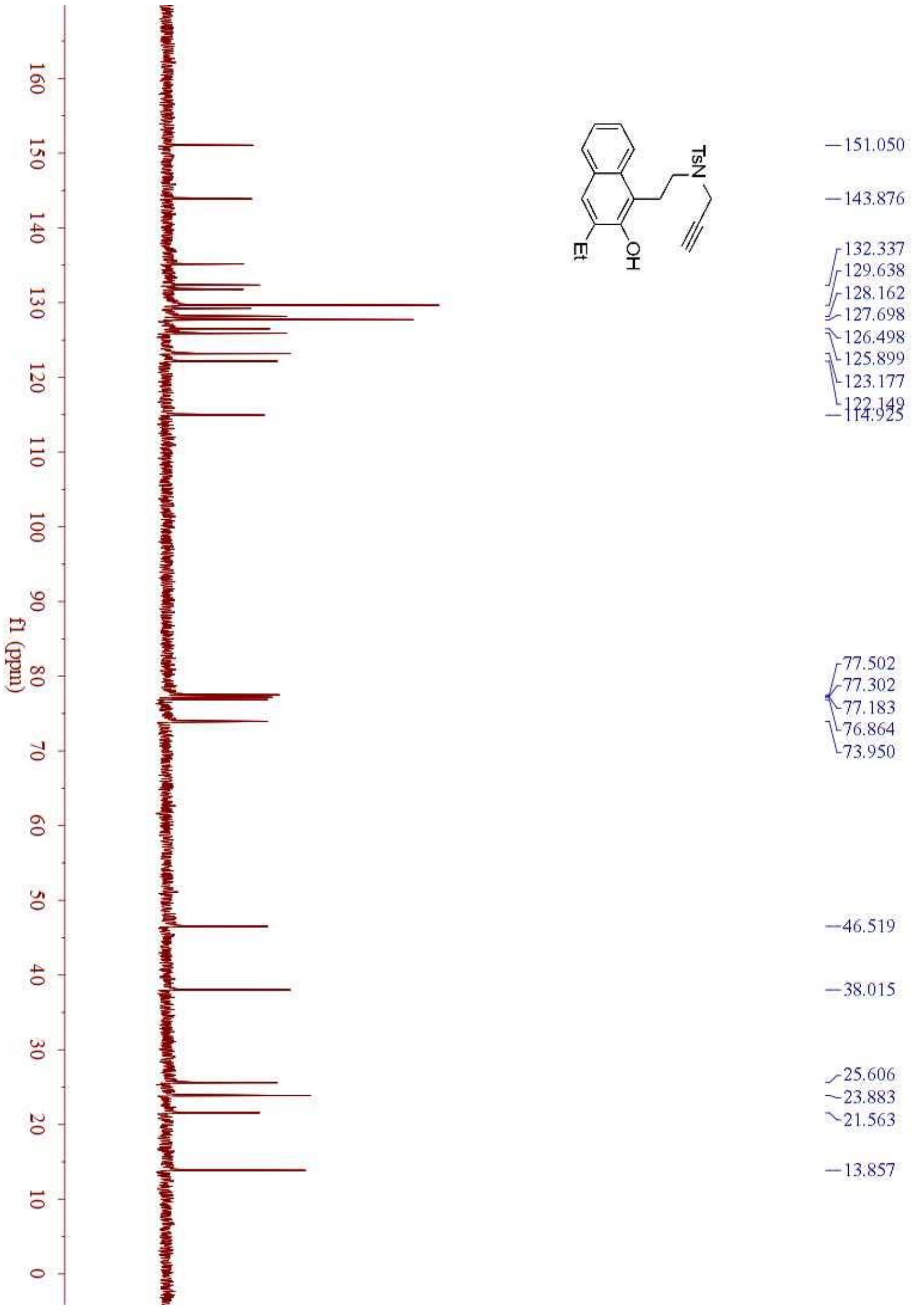


Figure $17{ }^{1} \mathrm{H}$ NMR spectrum of $\mathbf{1 i}\left(400 \mathrm{MHz}, \mathrm{CDCl}_{3}\right)$

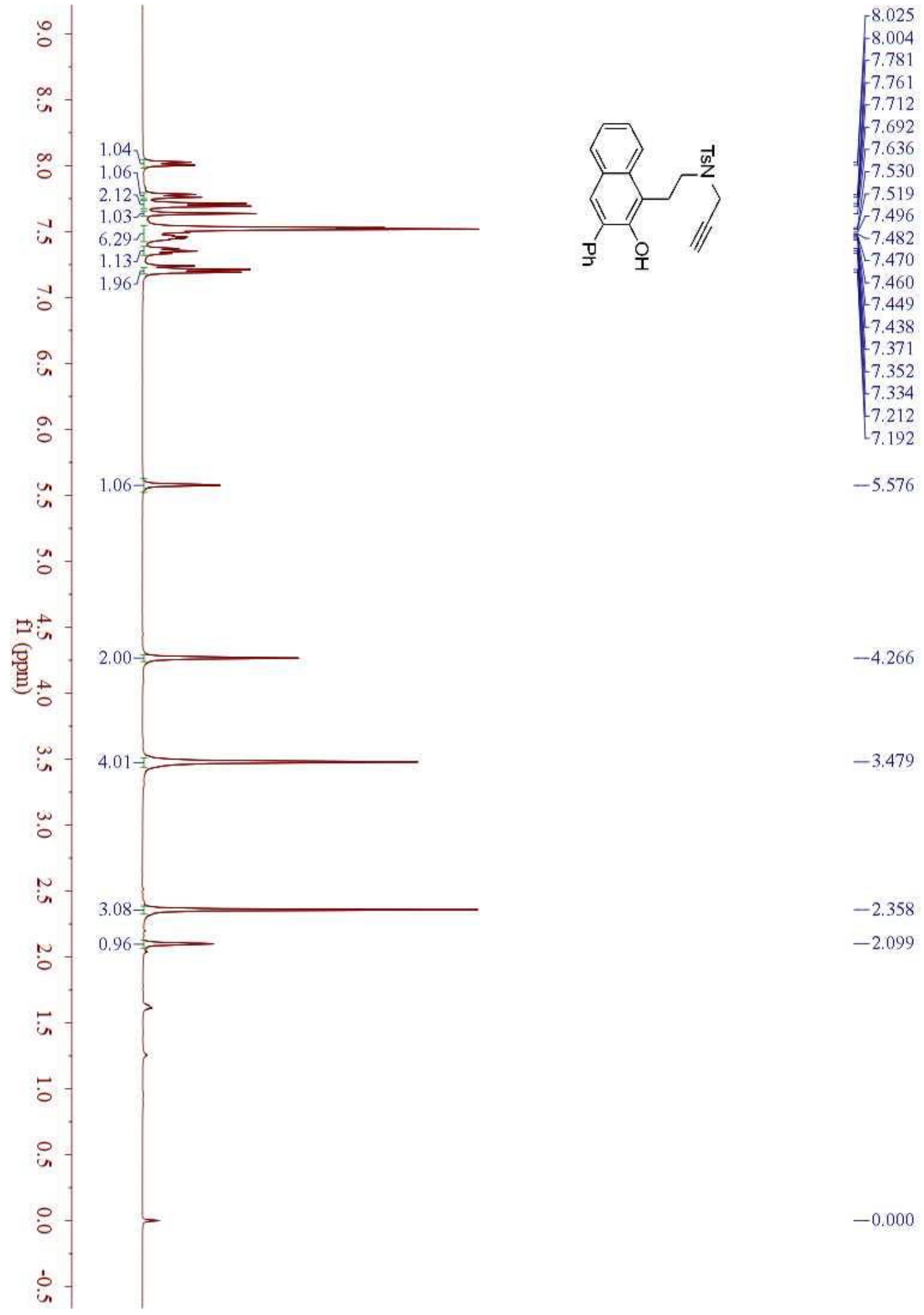


Figure $18{ }^{13} \mathrm{C}$ NMR spectrum of $1 \mathbf{i}\left(100 \mathrm{MHz}, \mathrm{CDCl}_{3}\right)$

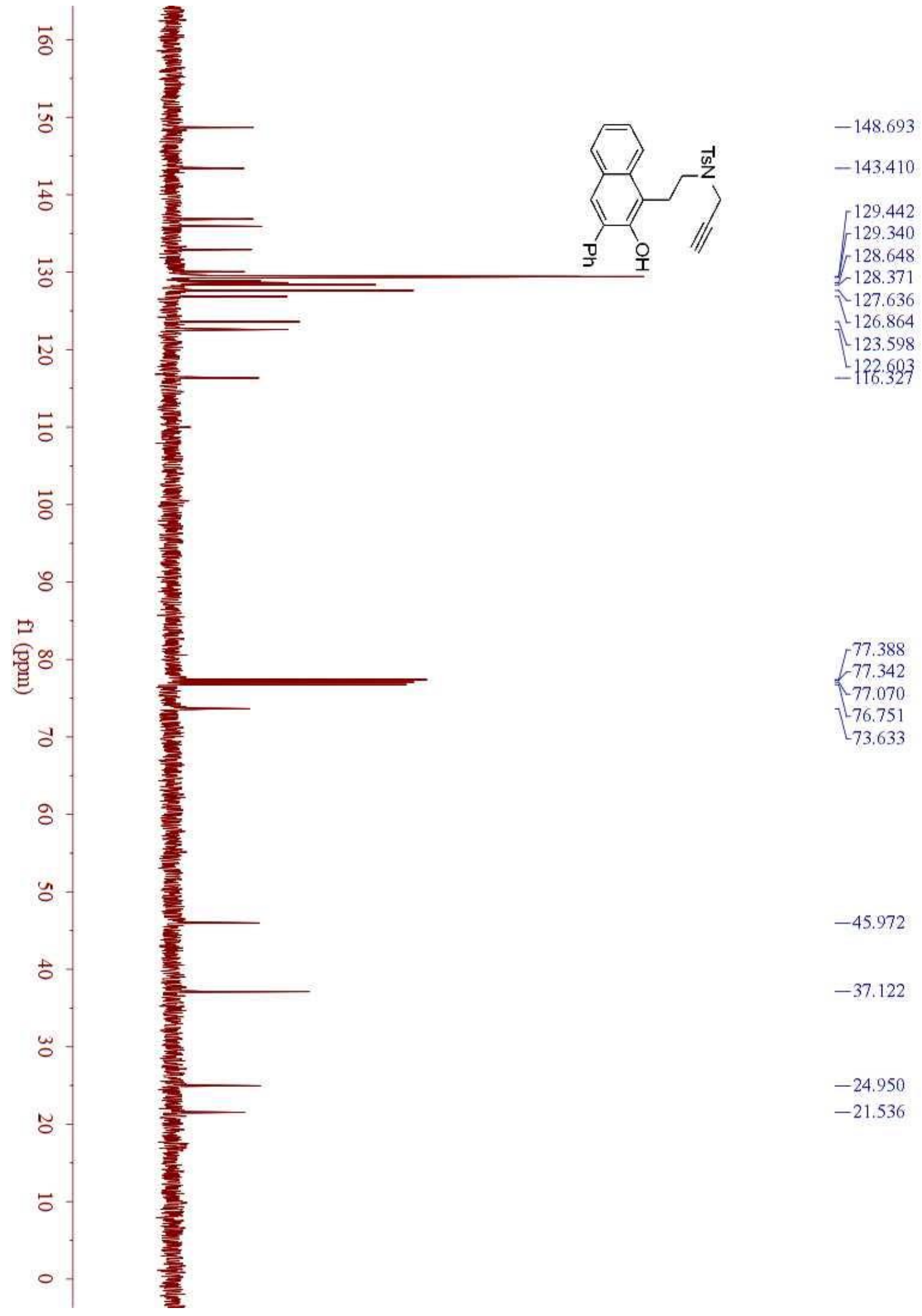


Figure $19{ }^{1} \mathrm{H}$ NMR spectrum of $\mathbf{1 j}\left(400 \mathrm{MHz}, \mathrm{CDCl}_{3}\right)$

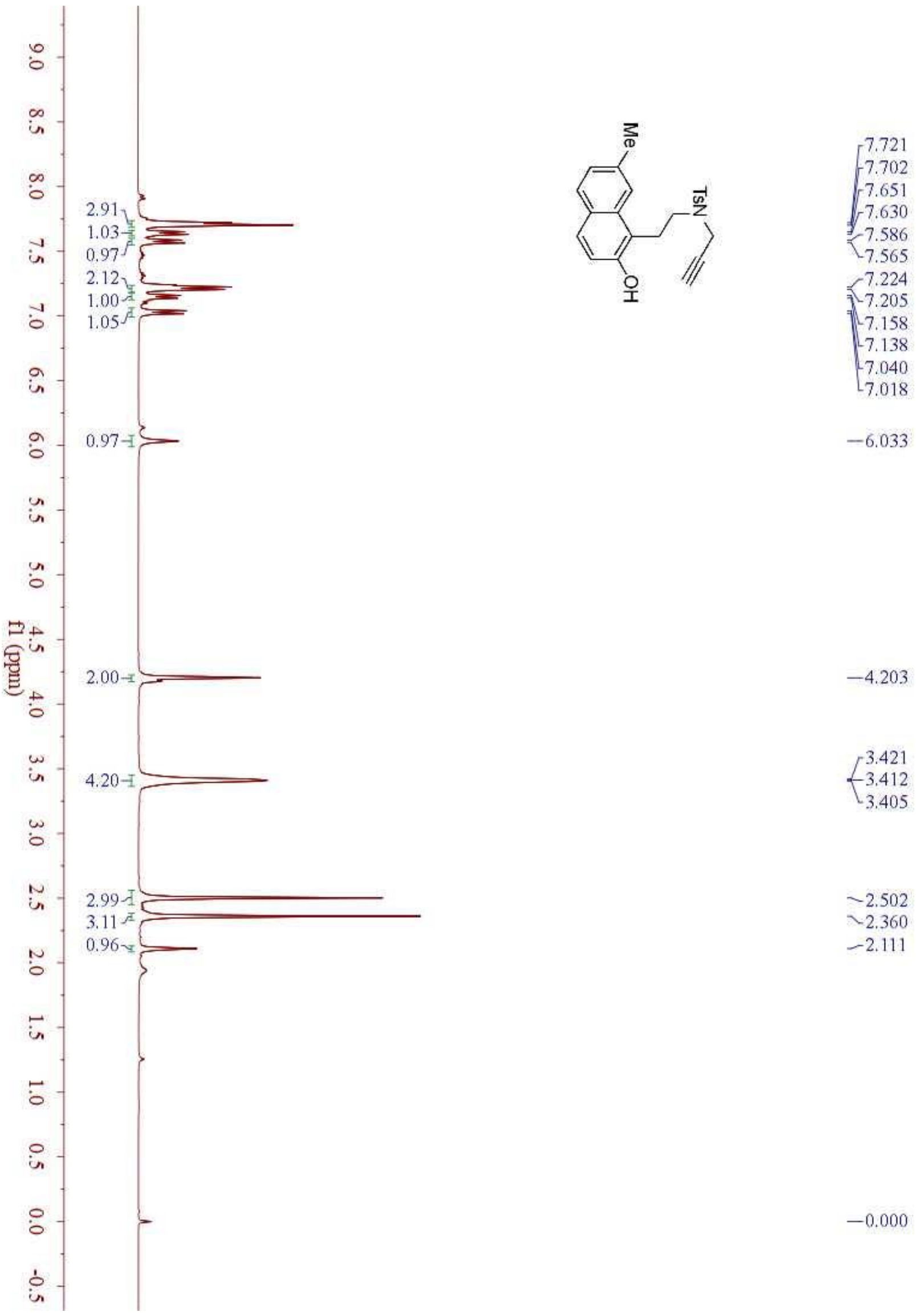


Figure $20{ }^{13} \mathrm{C}$ NMR spectrum of $\mathbf{1 j}\left(100 \mathrm{MHz}, \mathrm{CDCl}_{3}\right)$

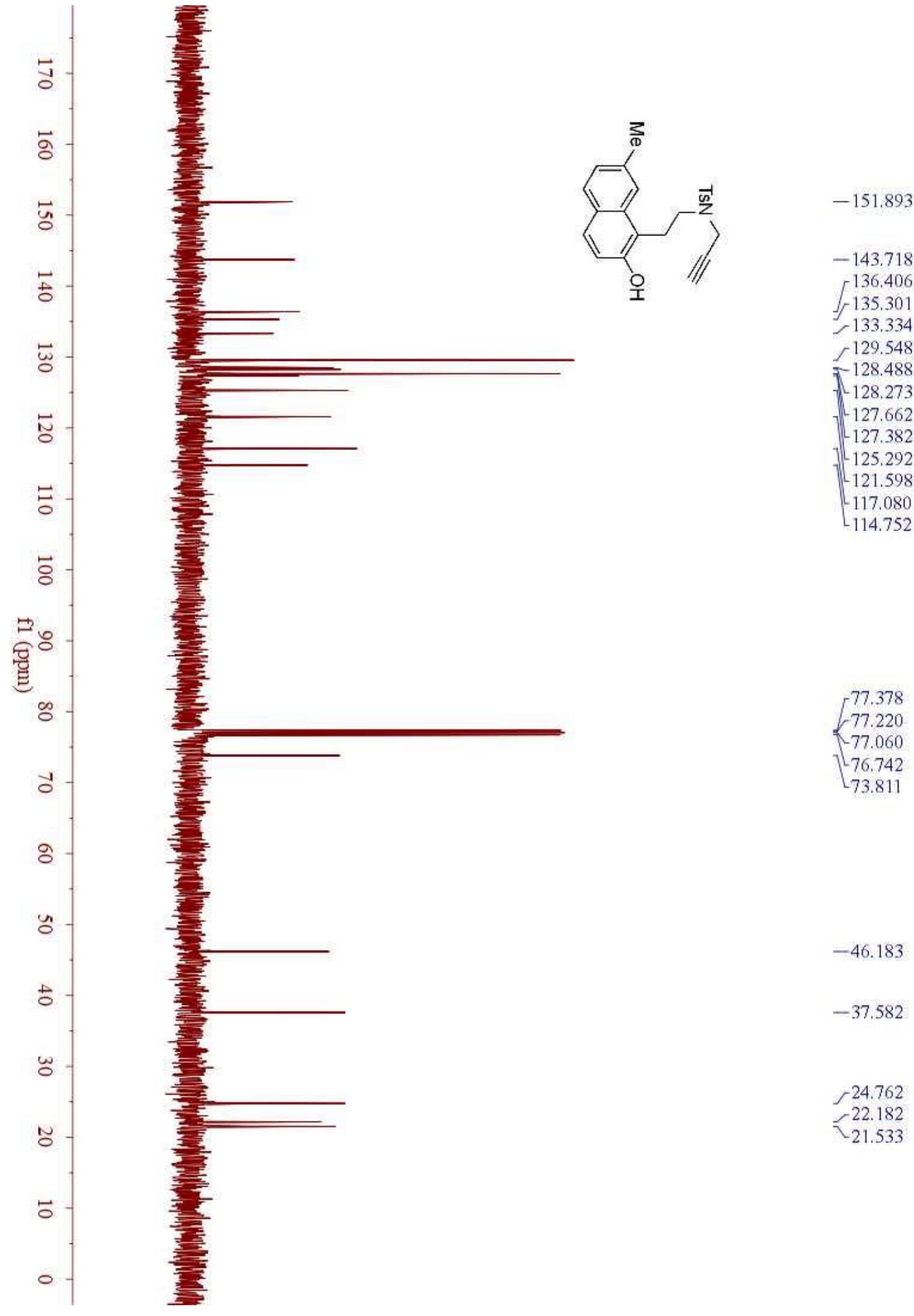


Figure $21{ }^{1} \mathrm{H}$ NMR spectrum of $\mathbf{1 k}\left(400 \mathrm{MHz}, \mathrm{CDCl}_{3}\right)$

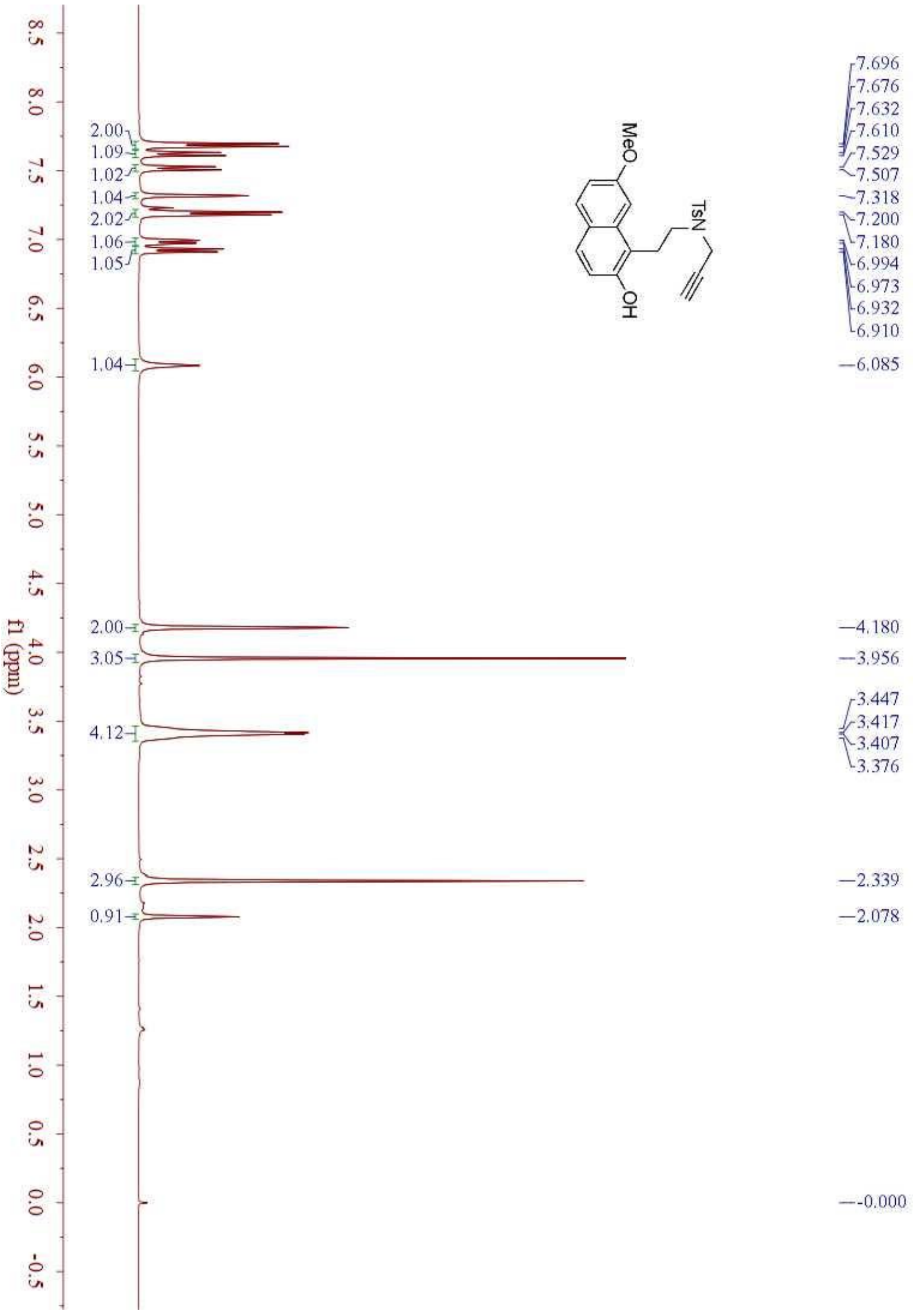


Figure $22{ }^{13} \mathrm{C}$ NMR spectrum of $\mathbf{1 k}\left(100 \mathrm{MHz}, \mathrm{CDCl}_{3}\right)$

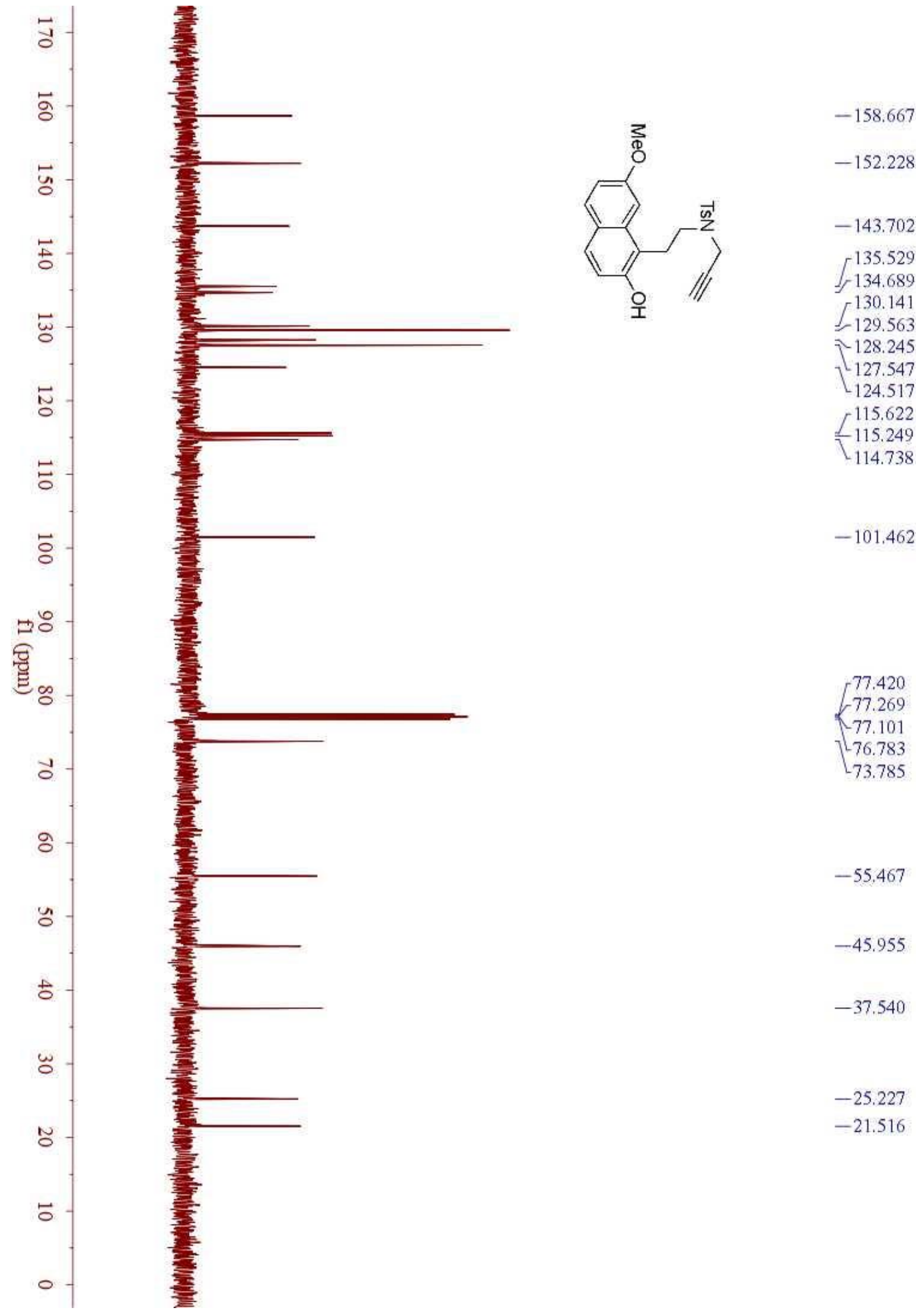


Figure $23{ }^{1} \mathrm{H}$ NMR spectrum of 11 (400 MHz, $\left.\mathrm{CDCl}_{3}\right)$

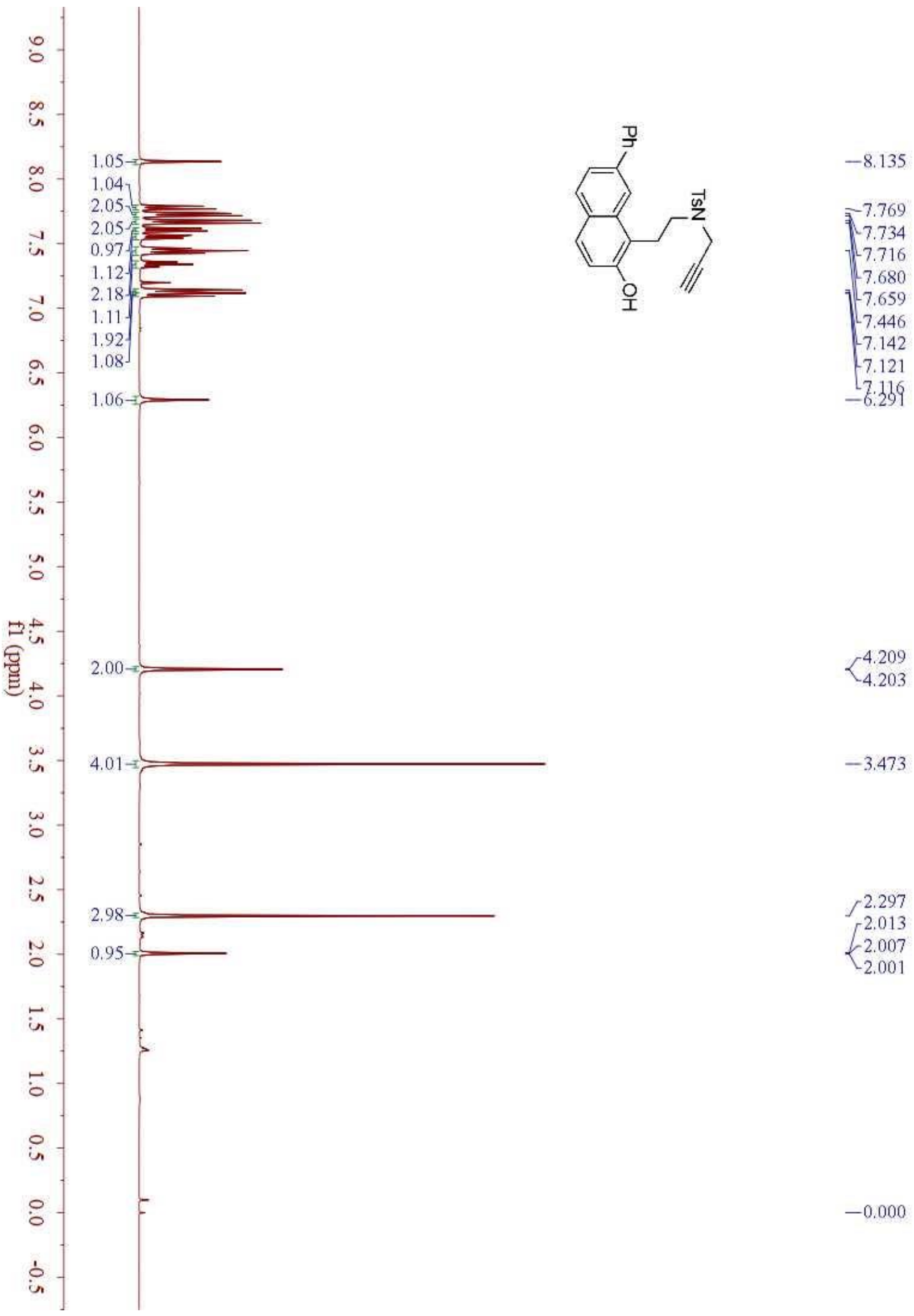


Figure $24{ }^{13} \mathrm{C}$ NMR spectrum of $11\left(100 \mathrm{MHz}, \mathrm{CDCl}_{3}\right)$

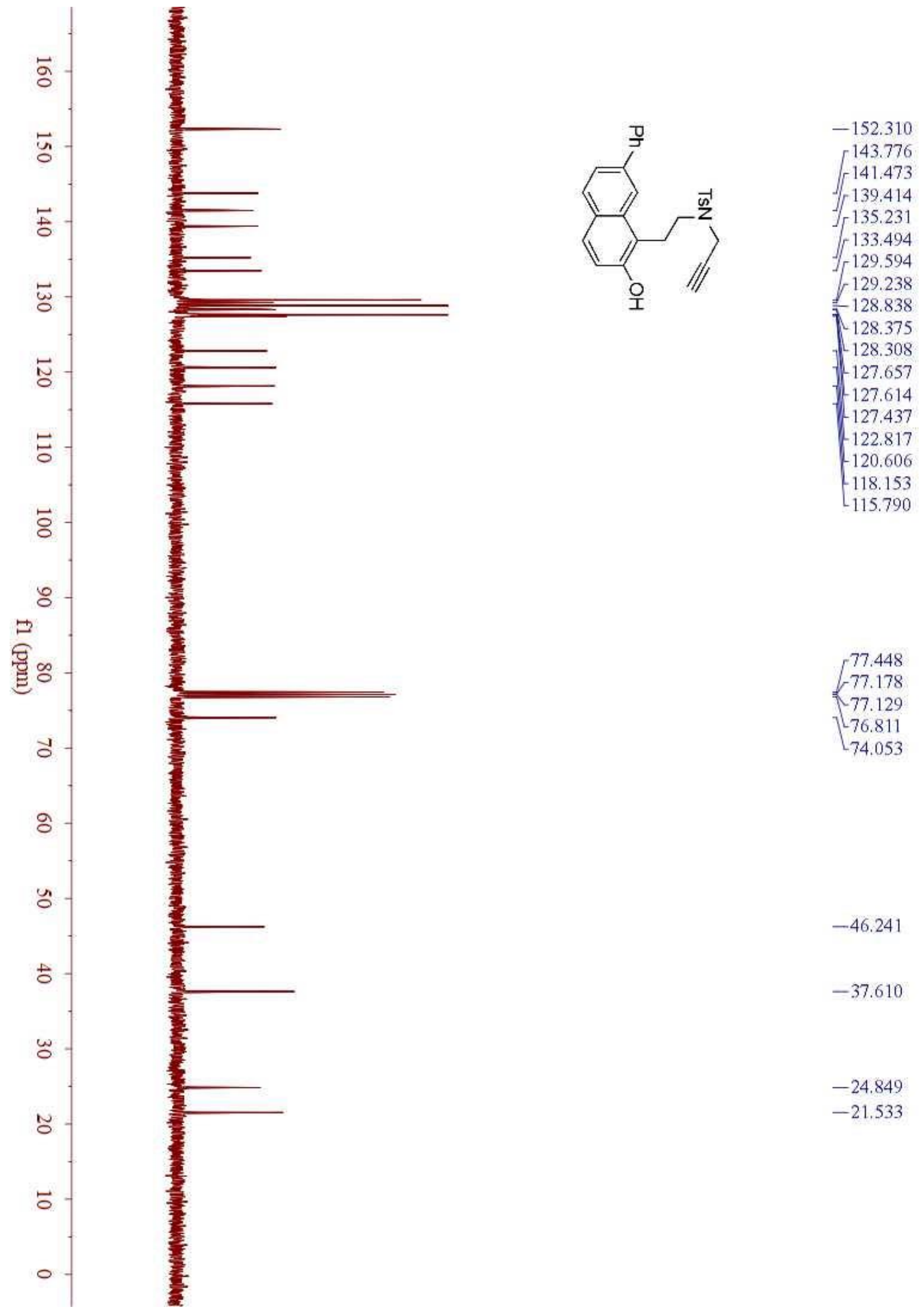


Figure $25{ }^{1} \mathrm{H}$ NMR spectrum of $\mathbf{1 m}\left(400 \mathrm{MHz}, \mathrm{CDCl}_{3}\right)$

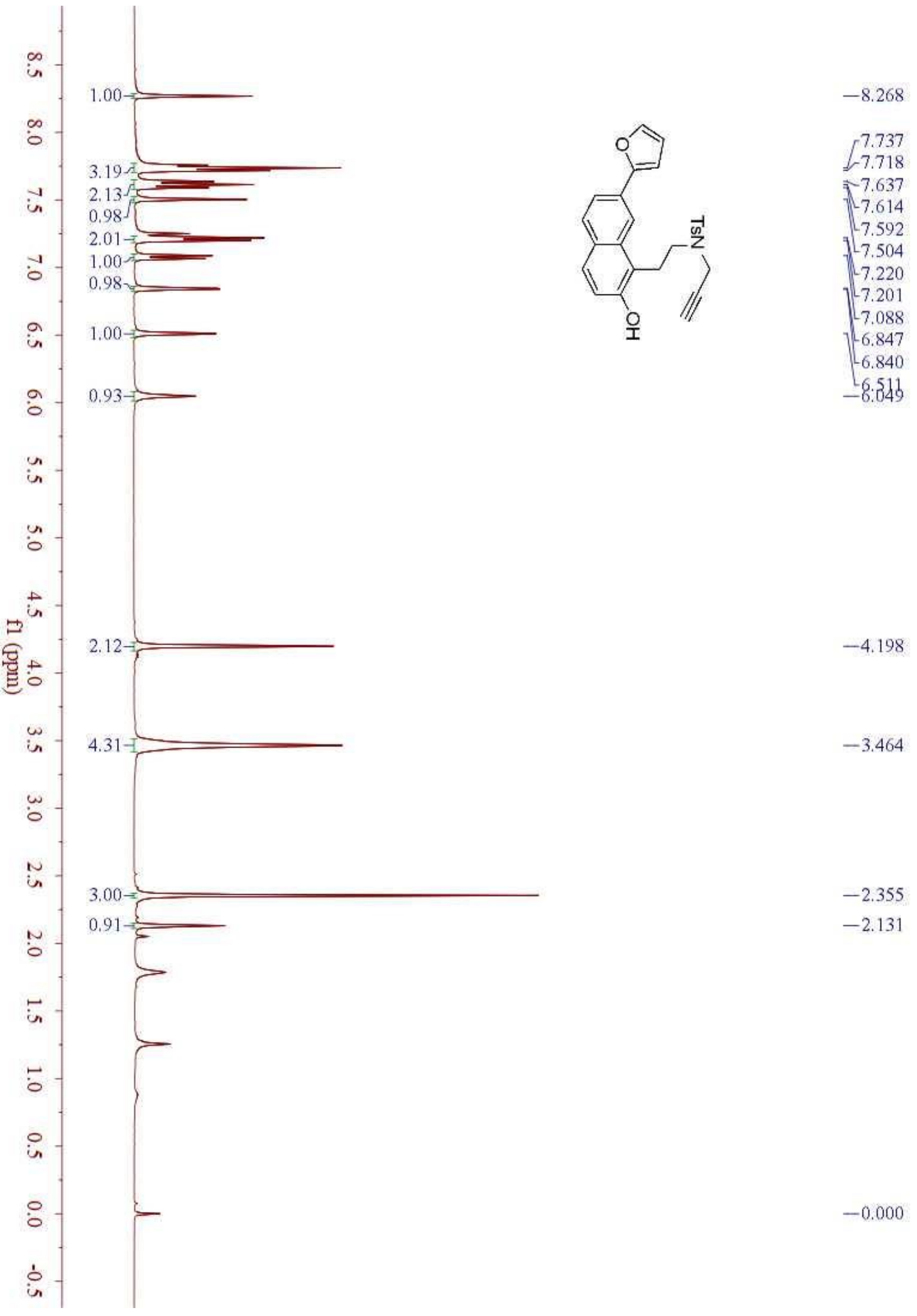


Figure $26{ }^{13} \mathrm{C}$ NMR spectrum of $\mathbf{1 m}\left(100 \mathrm{MHz}, \mathrm{CDCl}_{3}\right)$

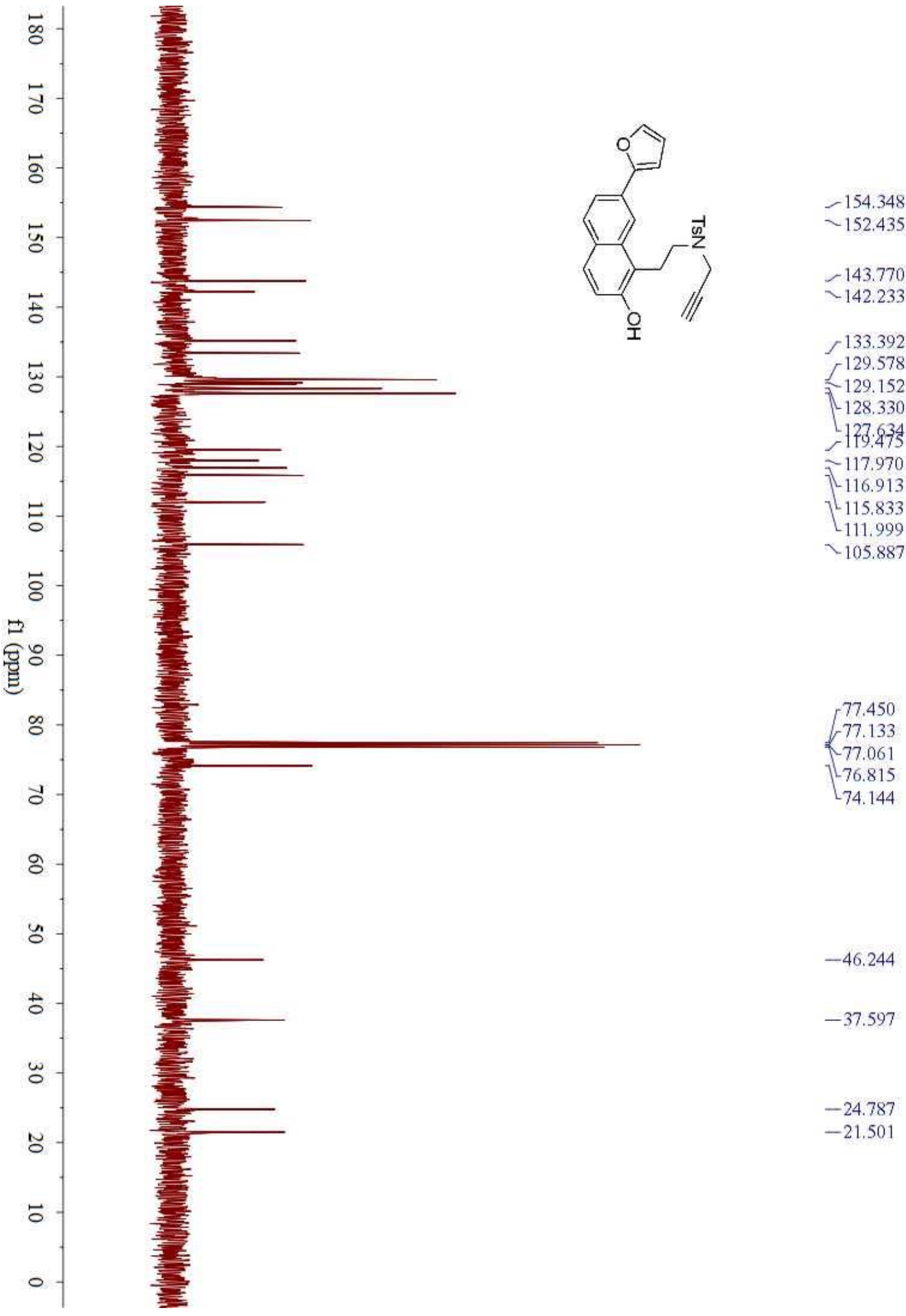


Figure $27{ }^{1} \mathrm{H}$ NMR spectrum of $\mathbf{1 n}\left(400 \mathrm{MHz}, \mathrm{CDCl}_{3}\right)$

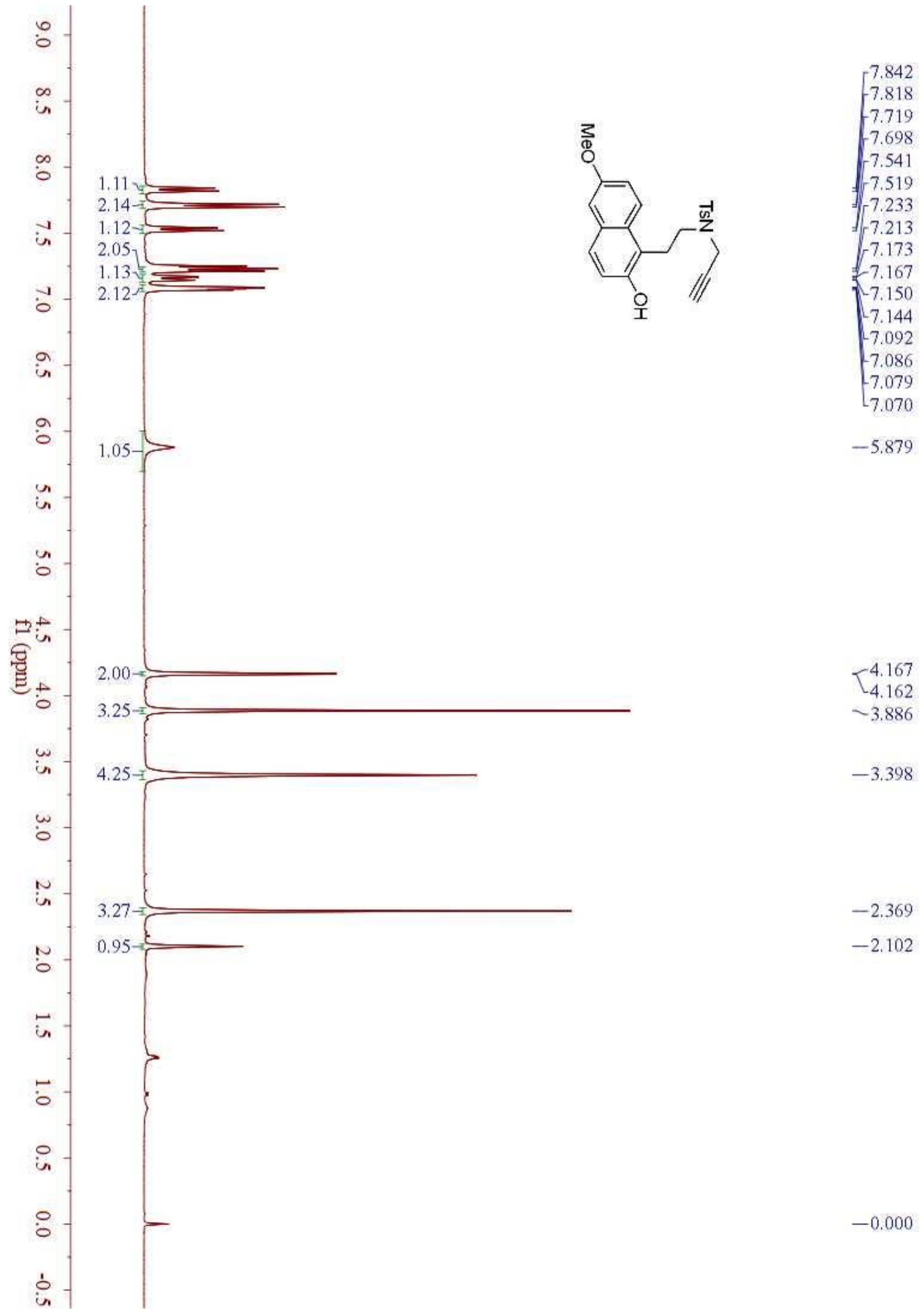


Figure $28{ }^{13} \mathrm{C}$ NMR spectrum of 1 n $\left(100 \mathrm{MHz}, \mathrm{CDCl}_{3}\right)$

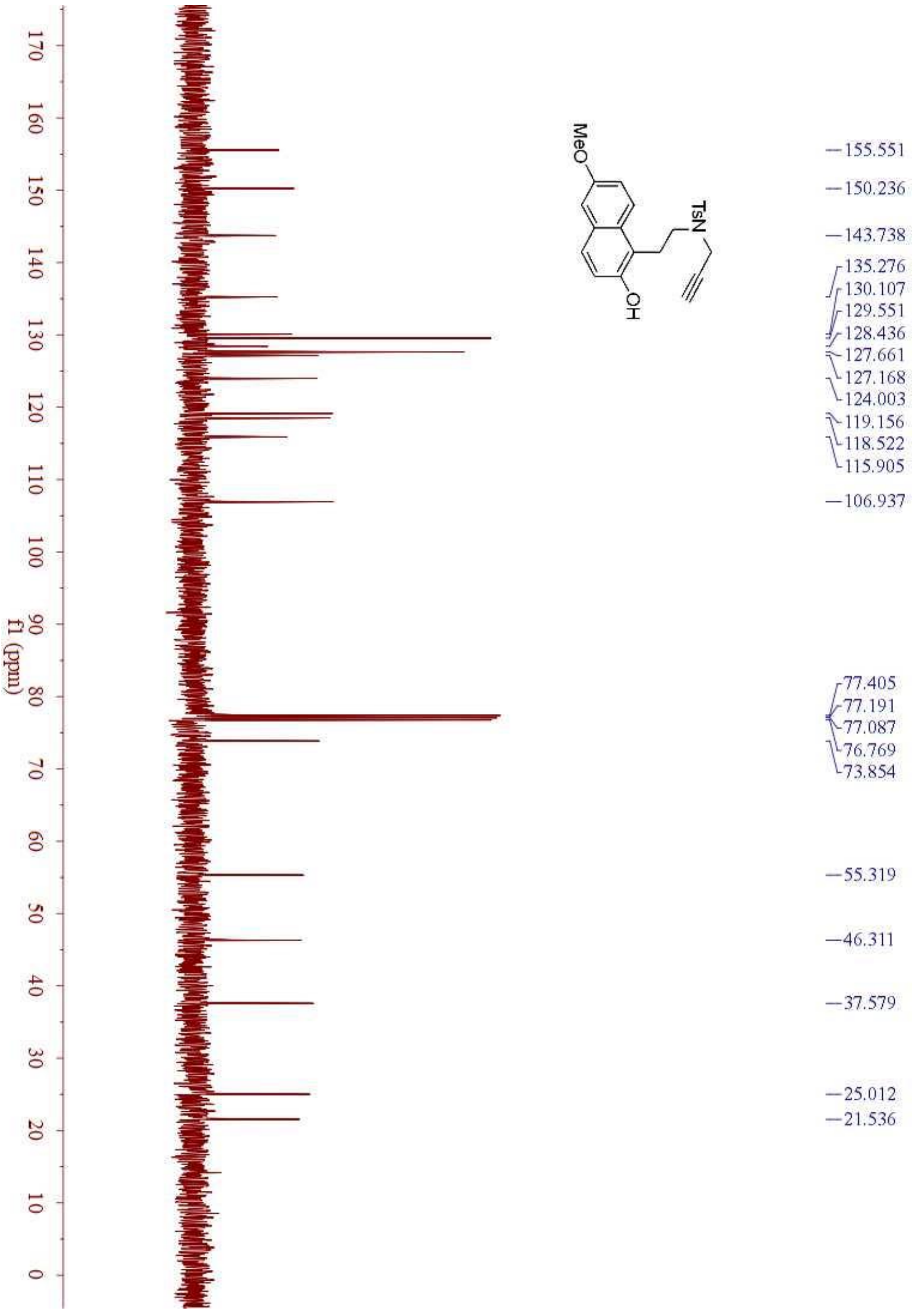


Figure $29{ }^{1} \mathrm{H}$ NMR spectrum of $10\left(400 \mathrm{MHz}, \mathrm{CDCl}_{3}\right)$

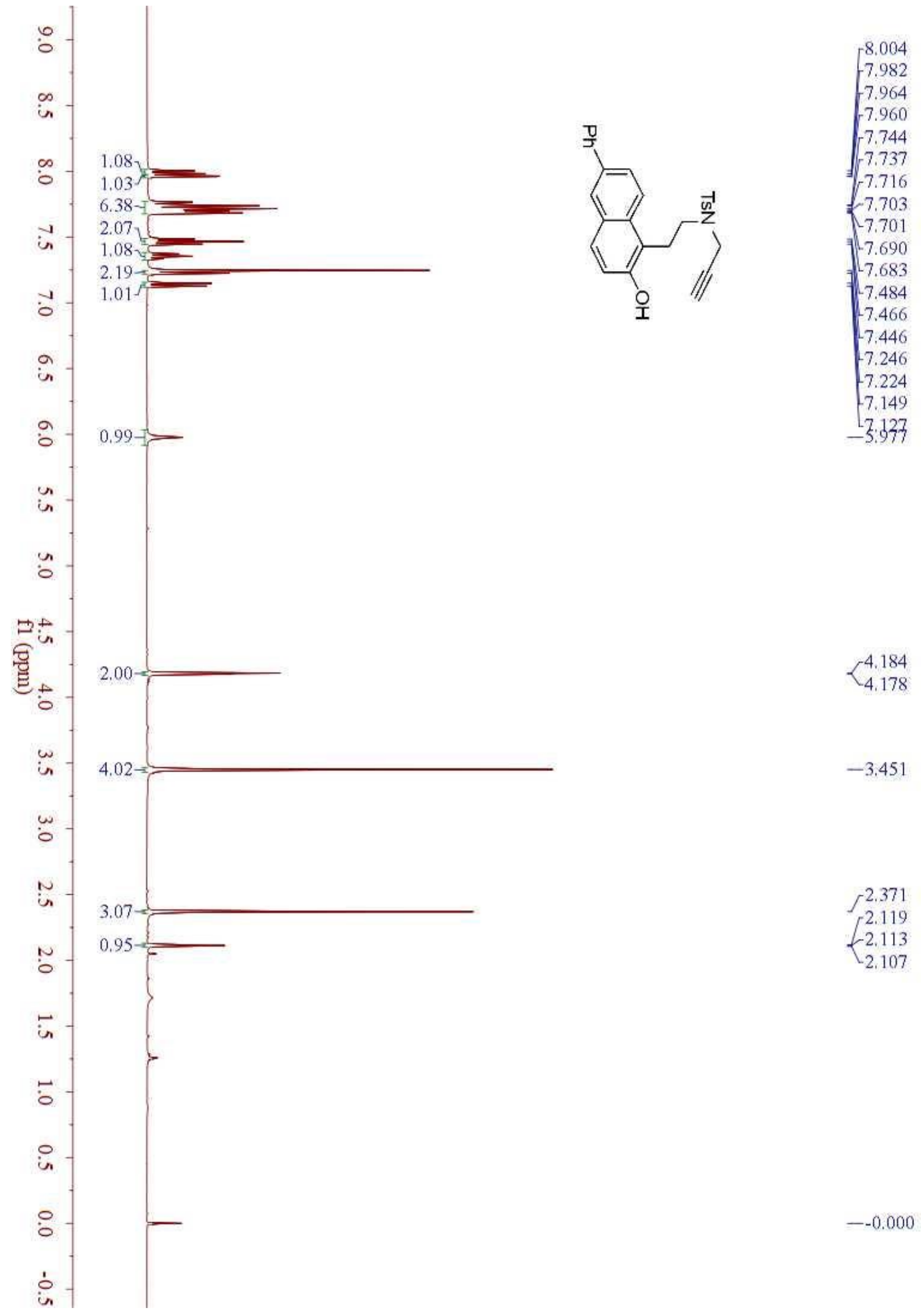


Figure $30{ }^{13} \mathrm{C}$ NMR spectrum of $10\left(100 \mathrm{MHz}, \mathrm{CDCl}_{3}\right)$

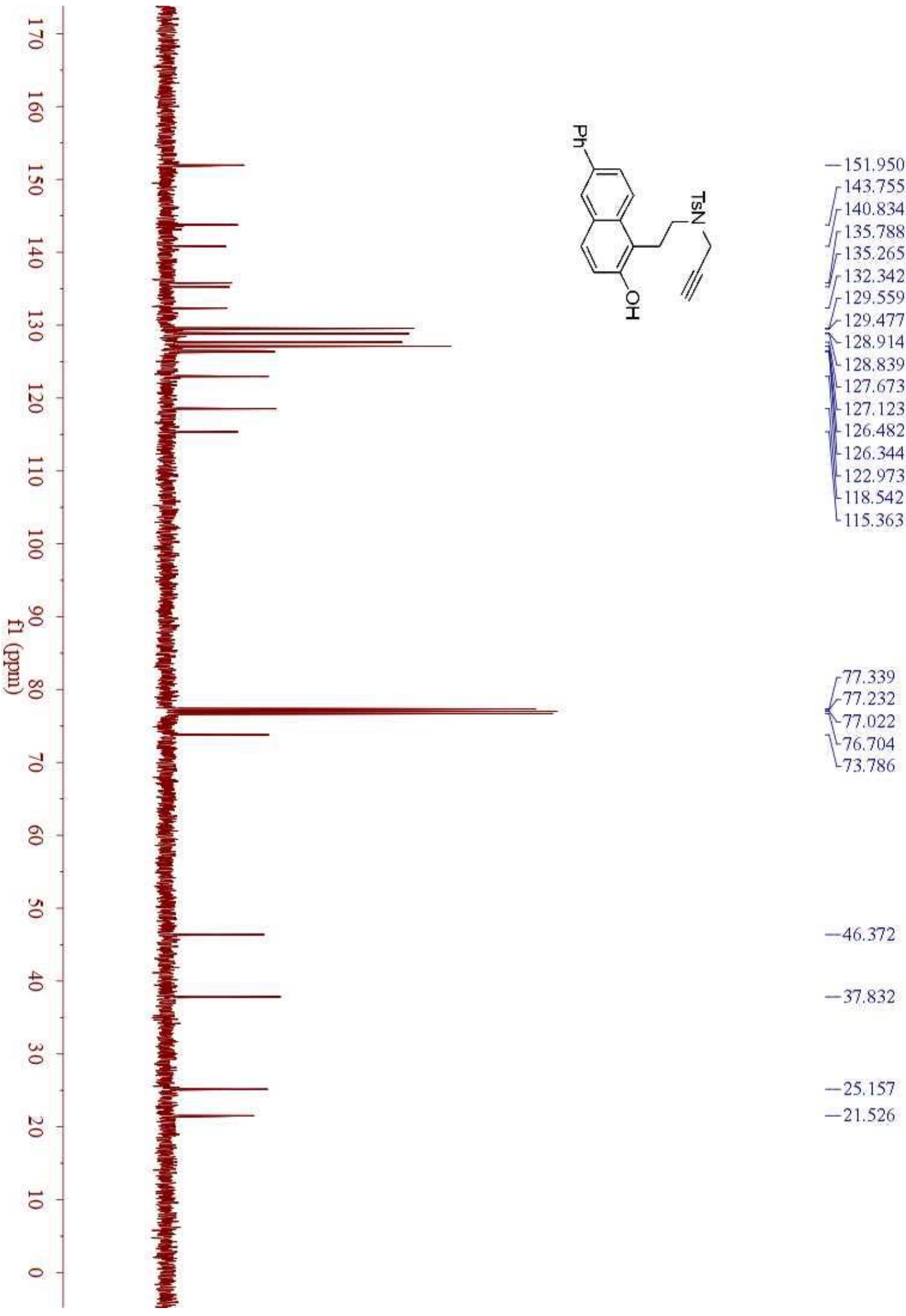


Figure $31{ }^{1} \mathrm{H}$ NMR spectrum of $\mathbf{1 p}\left(400 \mathrm{MHz}, \mathrm{CDCl}_{3}\right)$

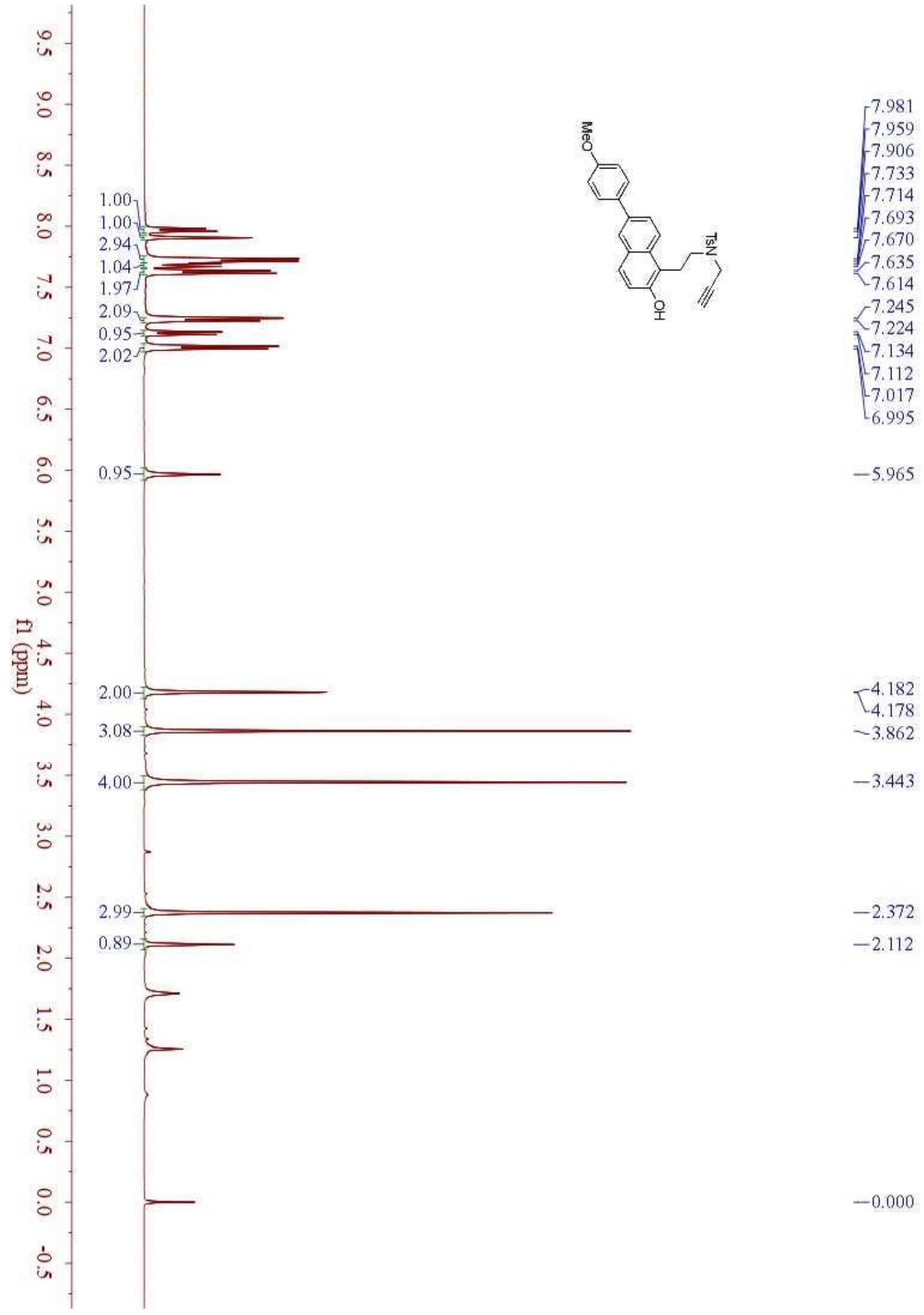


Figure $32{ }^{13} \mathrm{C}$ NMR spectrum of $\mathbf{1 p}\left(100 \mathrm{MHz}, \mathrm{CDCl}_{3}\right)$

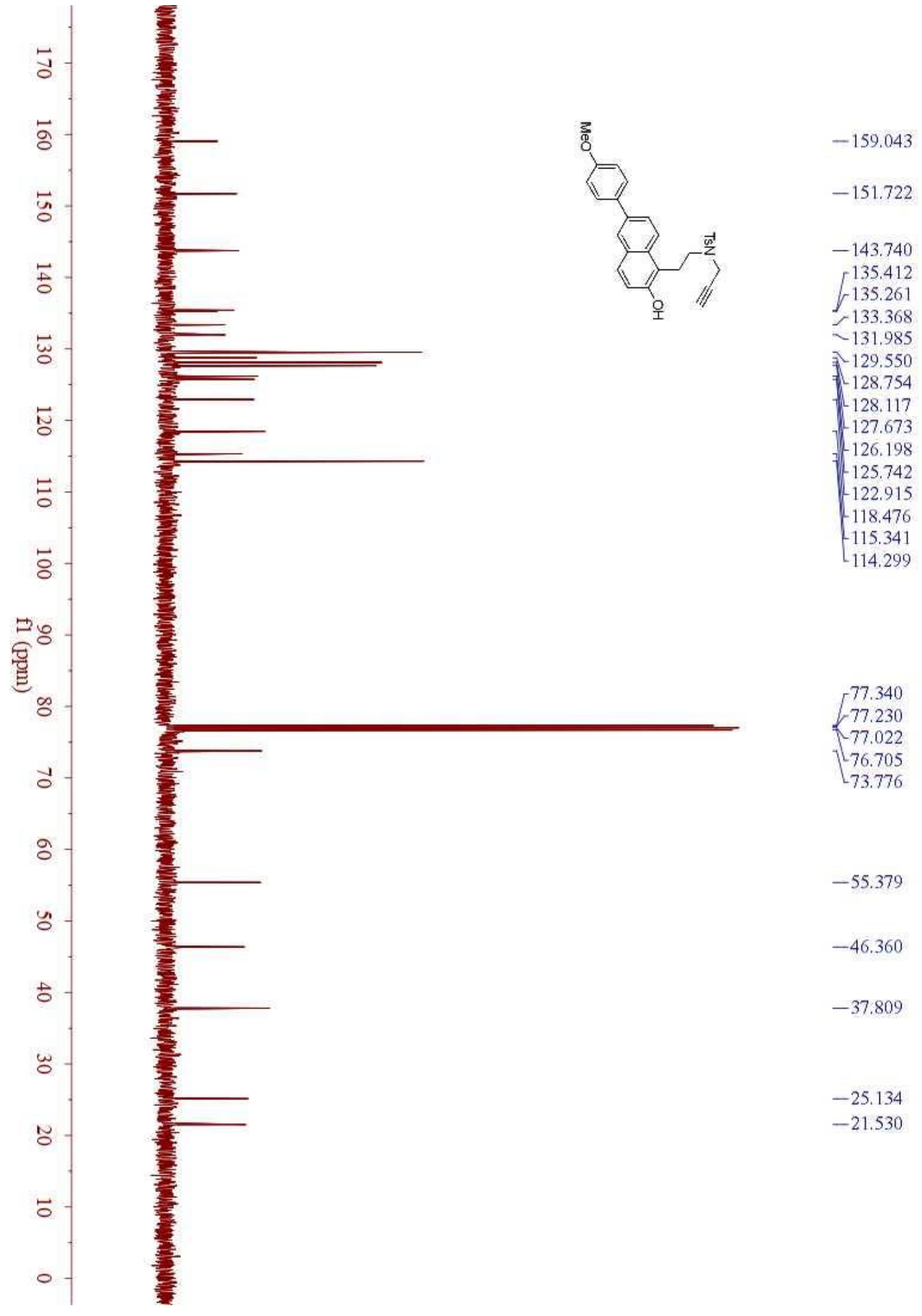


Figure $33{ }^{1} \mathrm{H}$ NMR spectrum of $\mathbf{1 q}$ (400 MHz, d6- Acetone)

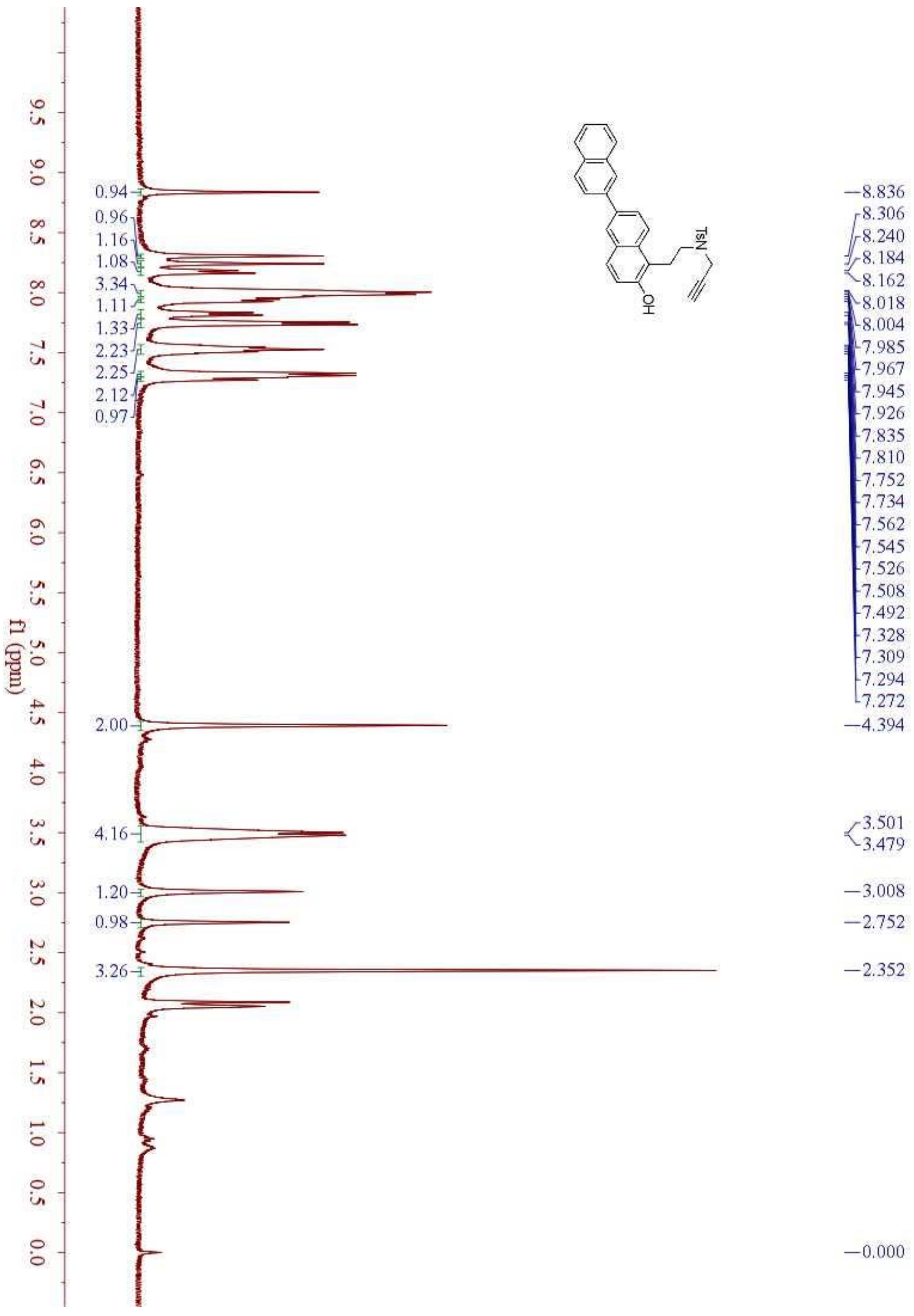


Figure $34{ }^{13} \mathrm{C}$ NMR spectrum of $\mathbf{1 q}\left(100 \mathrm{MHz}, \mathrm{d}_{6}\right.$ - Acetone $)$

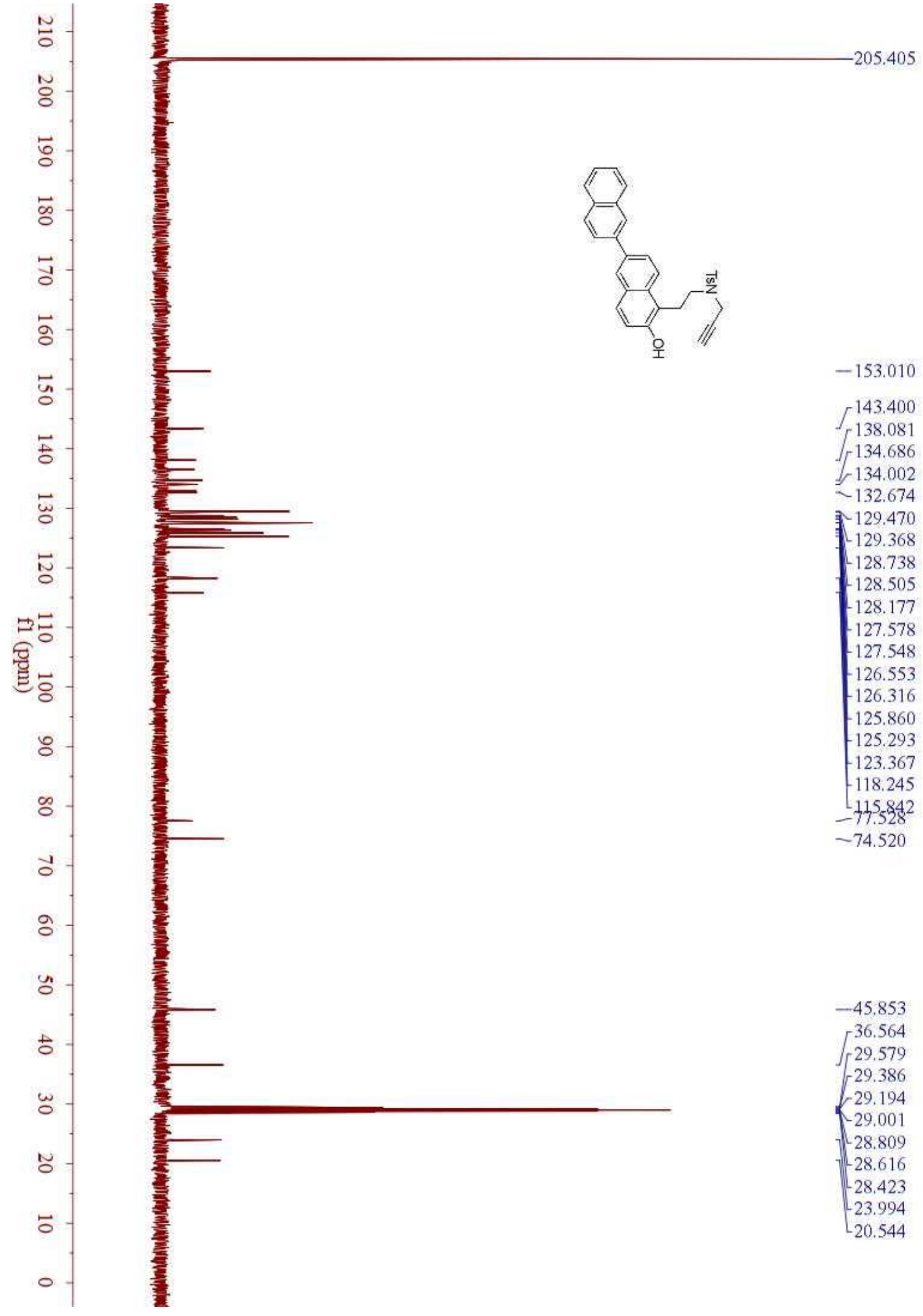


Figure $35{ }^{1} \mathrm{H}$ NMR spectrum of $1 r\left(400 \mathrm{MHz}, \mathrm{CDCl}_{3}\right)$

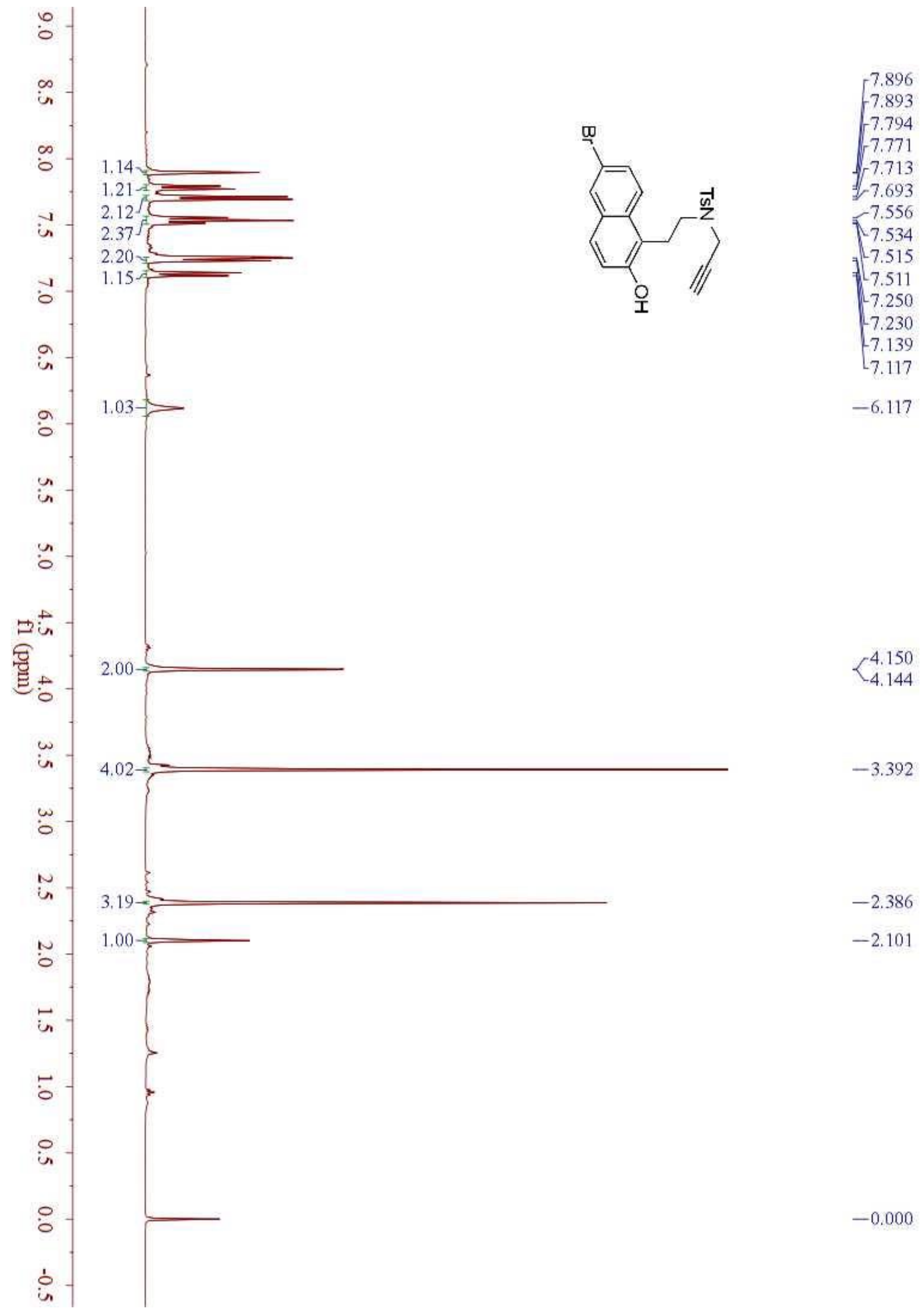


Figure $36{ }^{13} \mathrm{C} \mathrm{NMR}$ spectrum of $\operatorname{1r}\left(100 \mathrm{MHz}, \mathrm{CDCl}_{3}\right)$

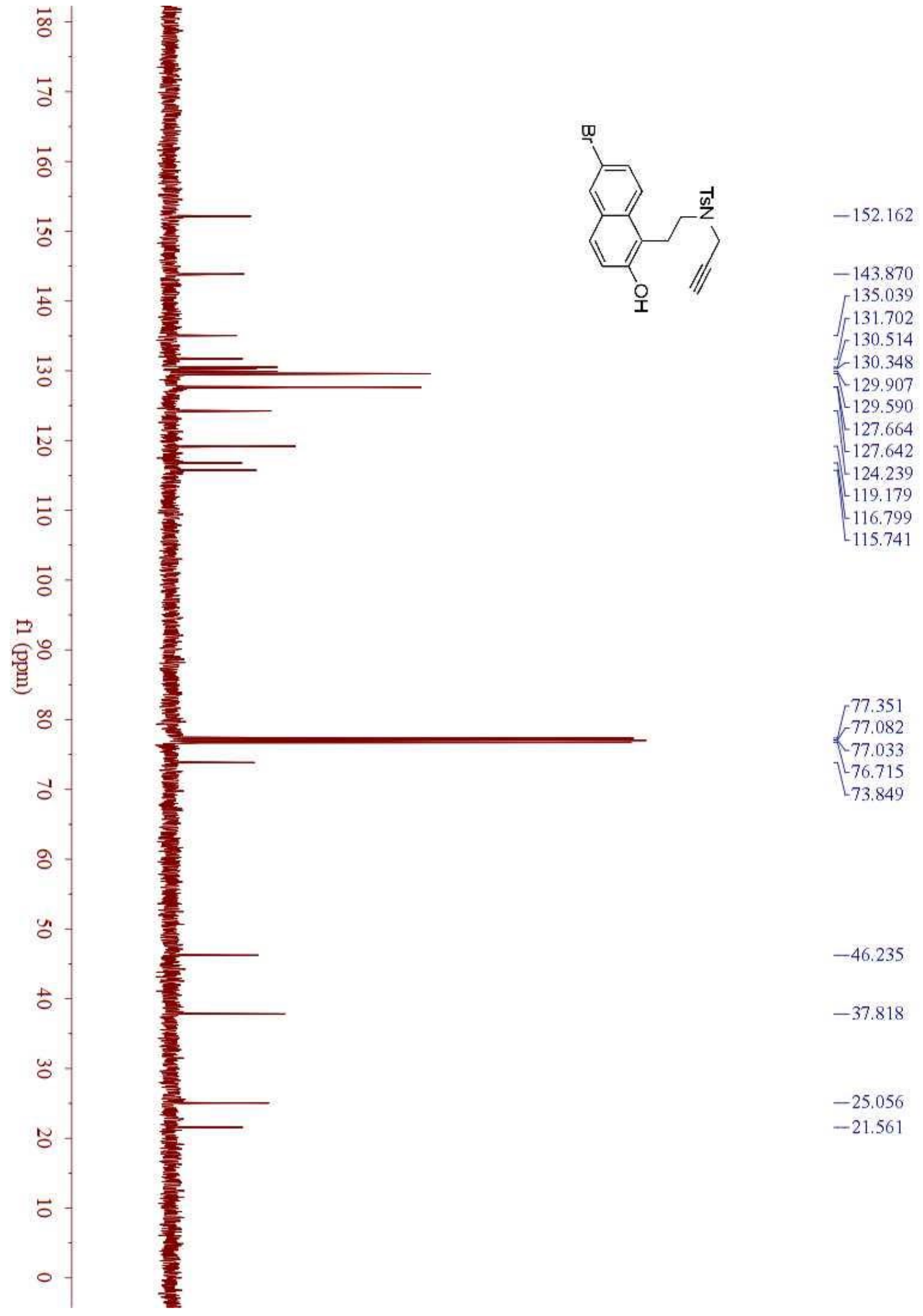


Figure $37{ }^{1} \mathrm{H}$ NMR spectrum of $\mathbf{2 a}\left(400 \mathrm{MHz}, \mathrm{CDCl}_{3}\right)$

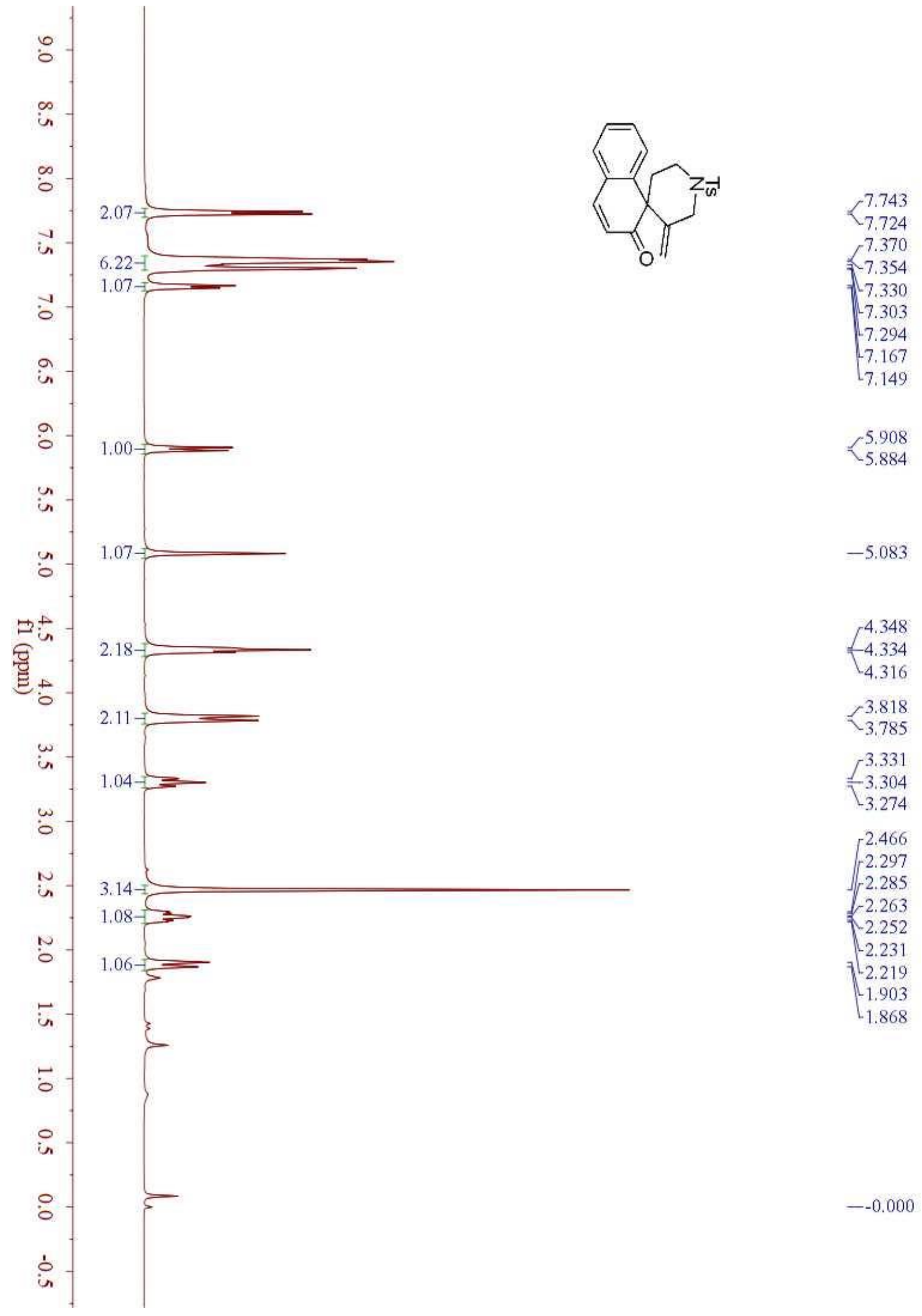


Figure $38{ }^{13} \mathrm{C}$ NMR spectrum of $\mathbf{2 a}\left(100 \mathrm{MHz}, \mathrm{CDCl}_{3}\right)$
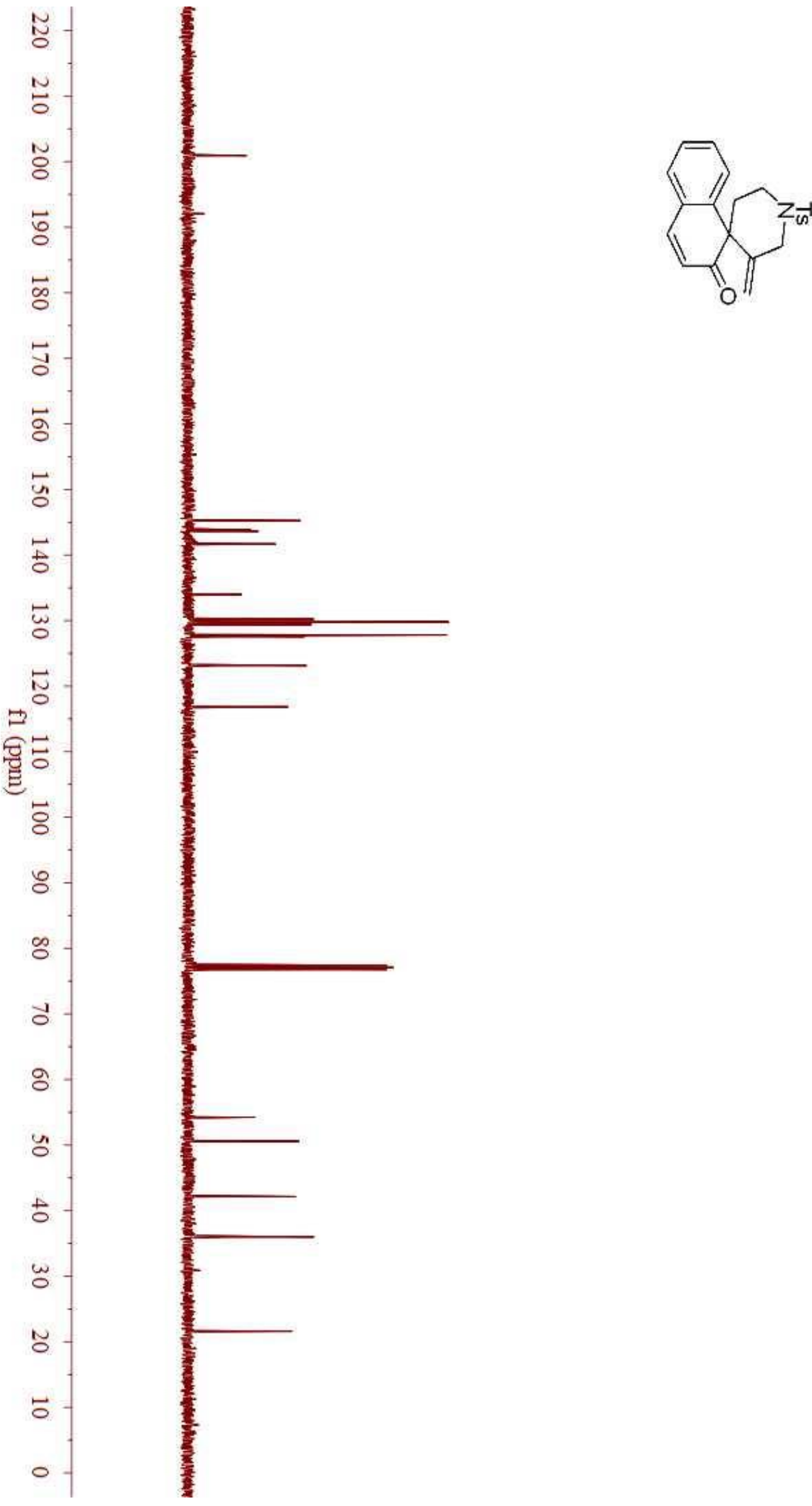

$-200.922$

$-145.227$

$-143.838$

143.631

1. 141.675

133.973

130.247

130.068

$-129.813$

129.770

$-129.380$

$-127.795$

$-127.549$

$-123.158$

$L_{116.819}$

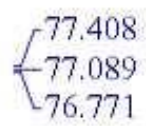

$-54.239$

$-50.599$

$-42.153$

$-35.999$

$-21.608$ 
Figure $39{ }^{1} \mathrm{H}$ NMR spectrum of $\mathbf{2 b}\left(400 \mathrm{MHz}, \mathrm{CDCl}_{3}\right)$

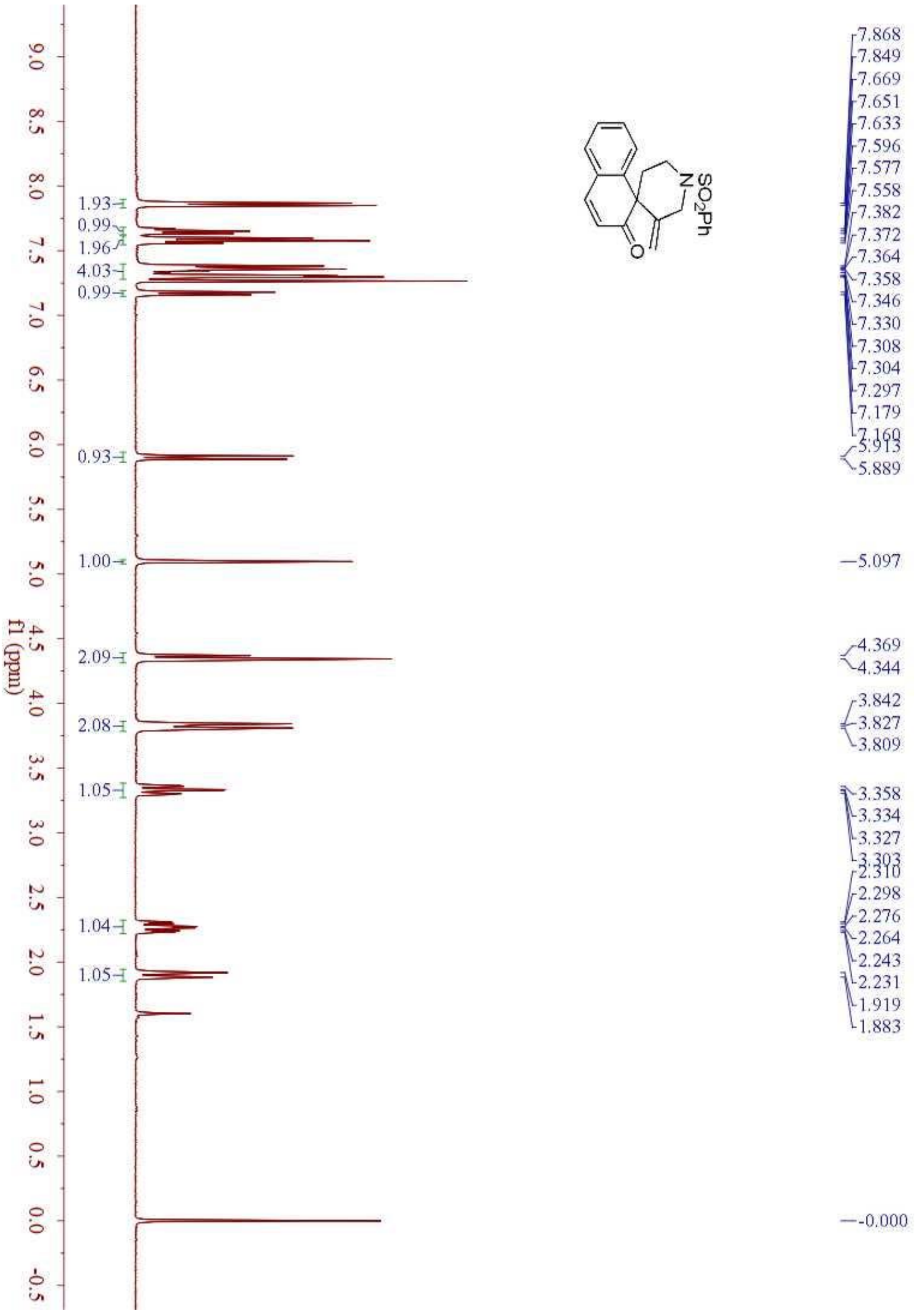


Figure $40{ }^{13} \mathrm{C}$ NMR spectrum of $\mathbf{2 b}\left(100 \mathrm{MHz}, \mathrm{CDCl}_{3}\right)$
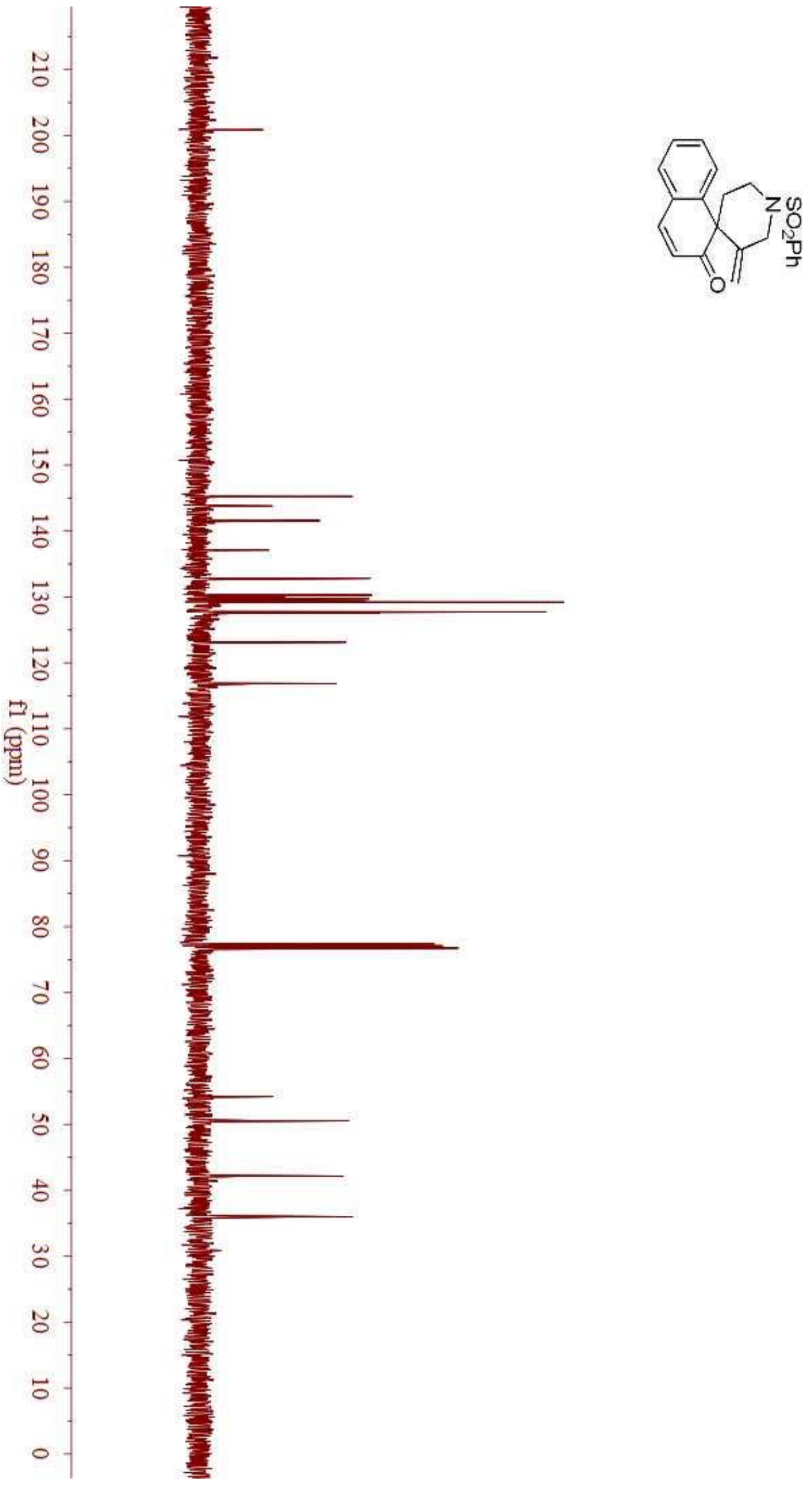
Figure $41{ }^{1} \mathrm{H}$ NMR spectrum of $2 \mathbf{c}\left(400 \mathrm{MHz}, \mathrm{CDCl}_{3}\right)$

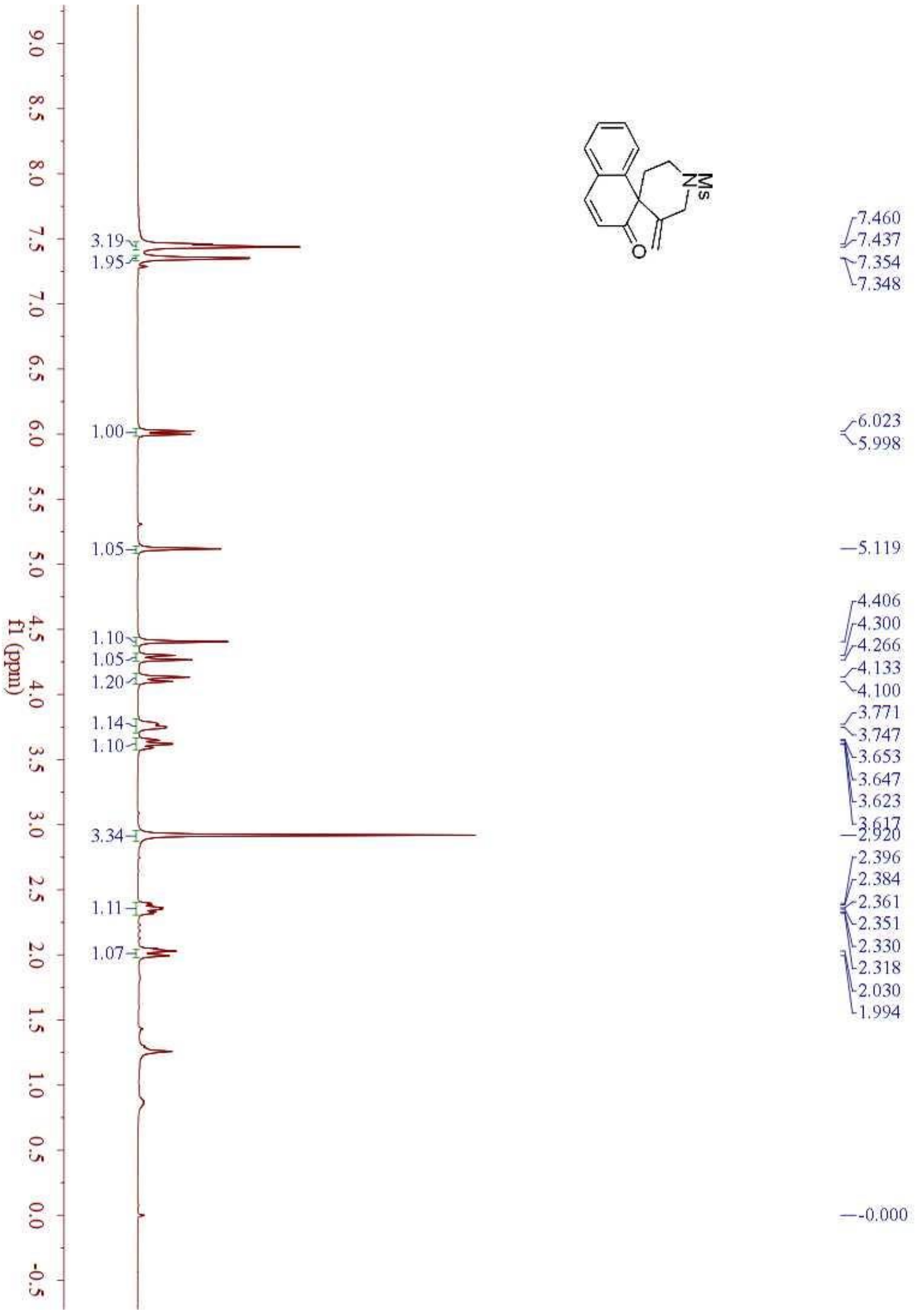


Figure $42{ }^{13} \mathrm{C}$ NMR spectrum of $2 \mathrm{c}\left(100 \mathrm{MHz}, \mathrm{CDCl}_{3}\right)$

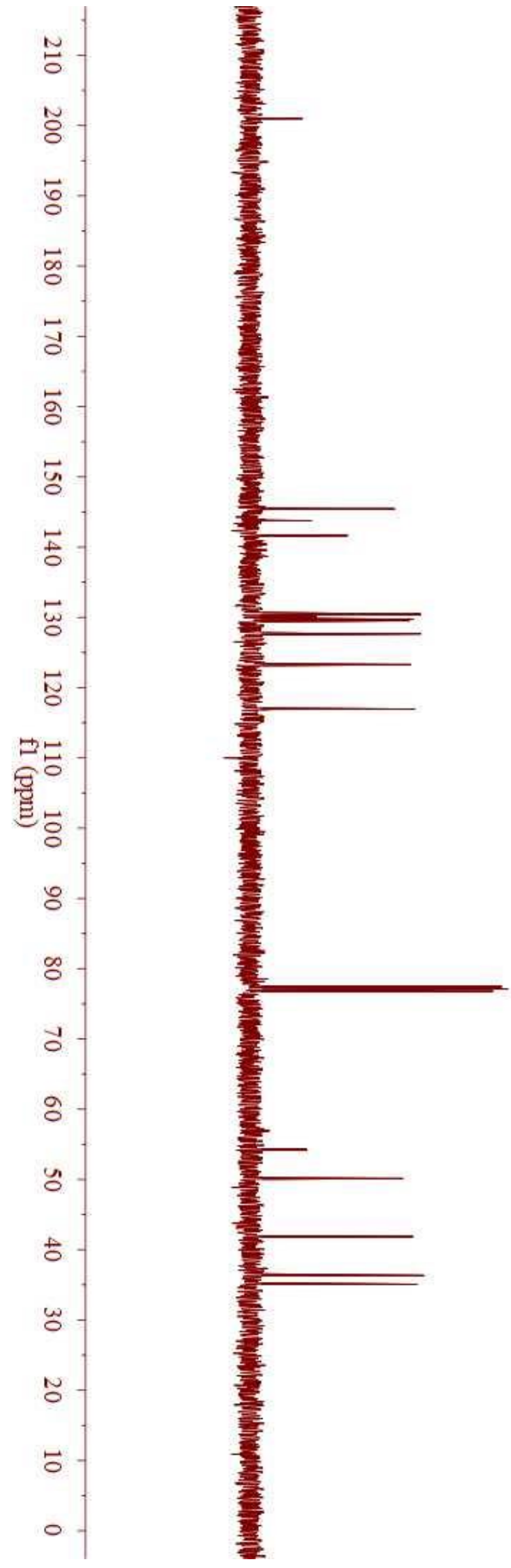

$-200.998$

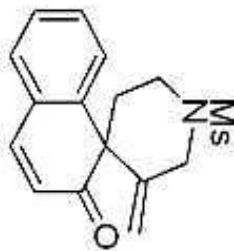

$\Gamma^{145.435}$

$-143.755$

$\chi_{141.652}$

130.447

$-130.085$

$-129.716$

$-129.510$

$-127.669$

- 123.275

$-116.943$

77.416

$-77.097$

$-76.779$

$-54.269$

$-50.135$

$-41.841$

$-36.324$

$-35.079$ 
Figure $43{ }^{1} \mathrm{H}$ NMR spectrum of $2 \mathbf{d}\left(400 \mathrm{MHz}, \mathrm{CDCl}_{3}\right)$

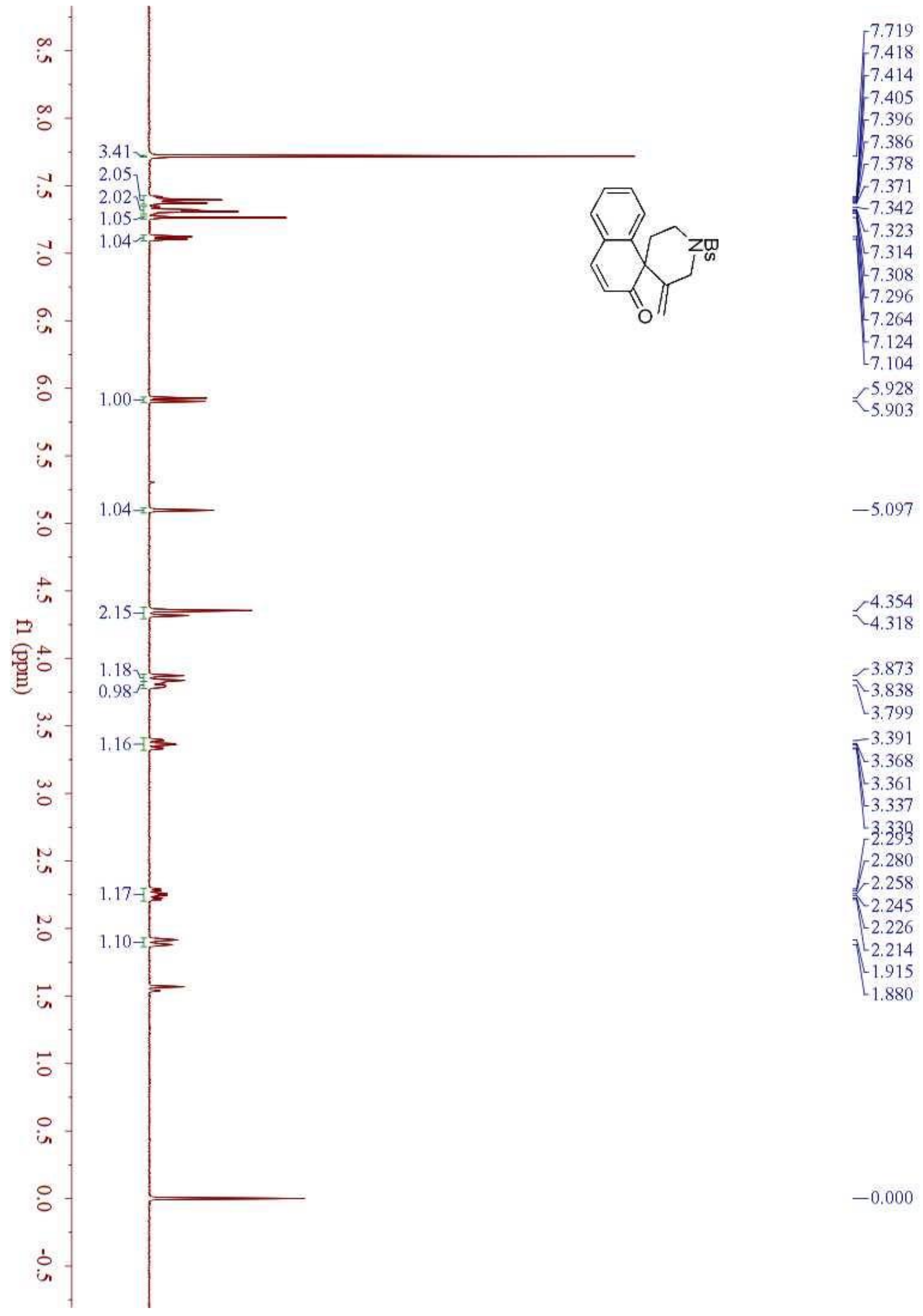


Figure $44{ }^{13} \mathrm{C}$ NMR spectrum of $2 d\left(100 \mathrm{MHz}, \mathrm{CDCl}_{3}\right)$

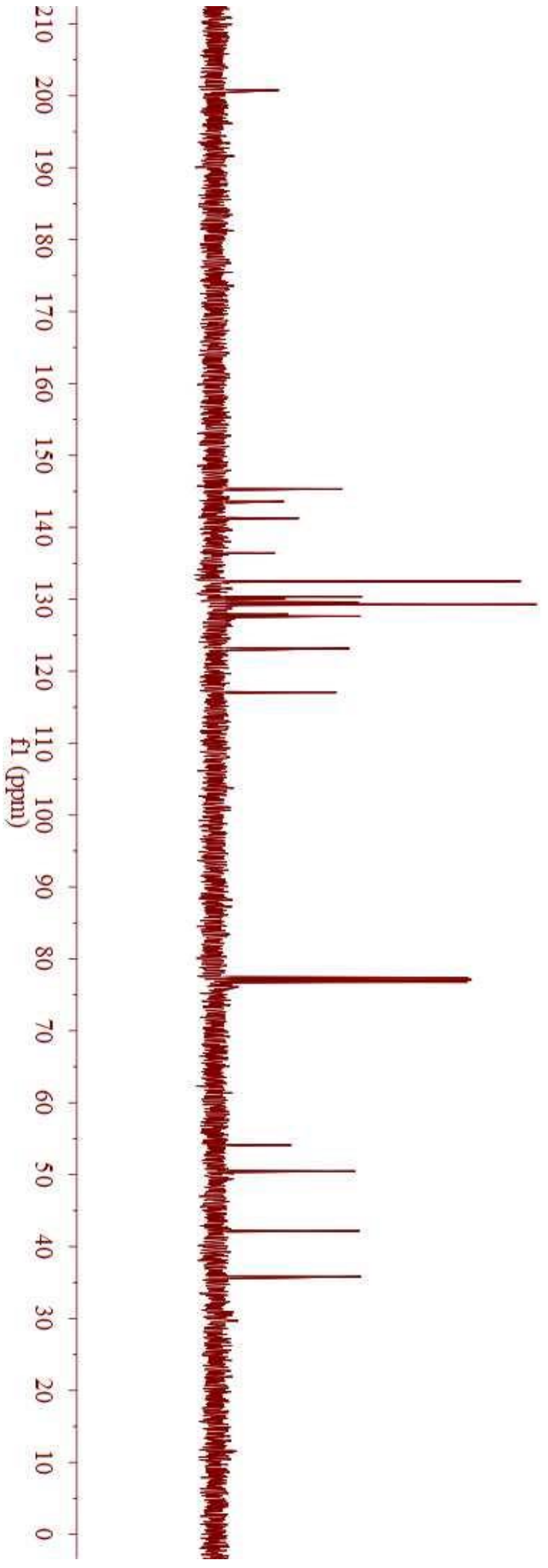

$-200.779$
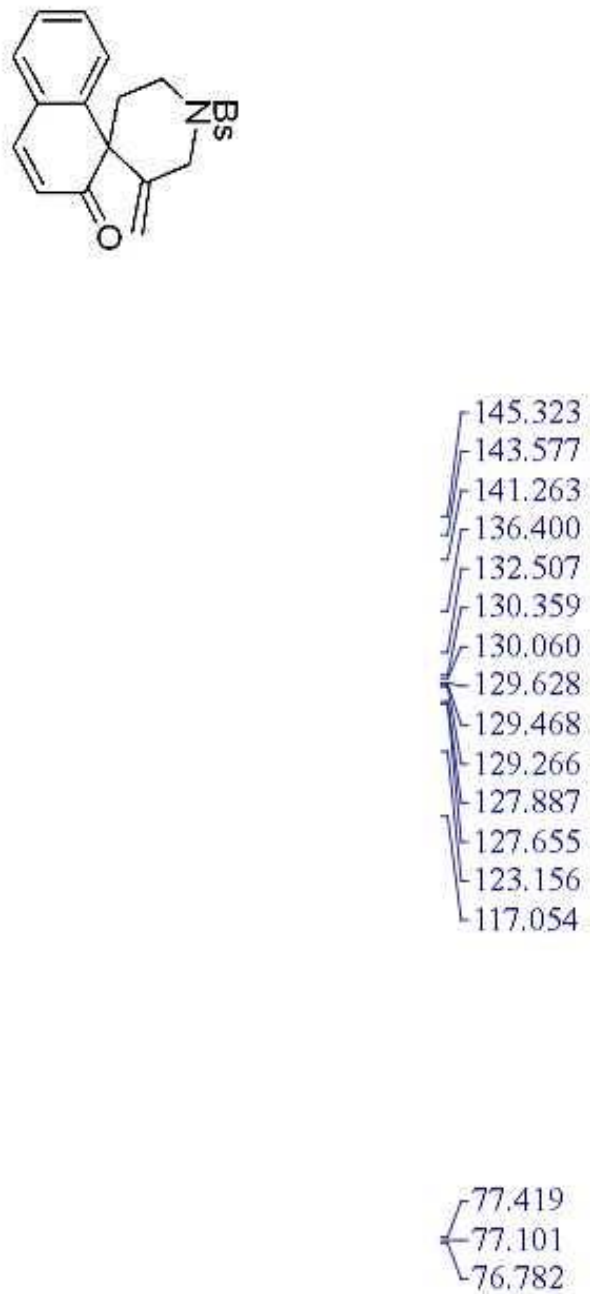

-54.127
-50.476

$-42.161$

$-35.809$ 
Figure $45{ }^{1} \mathrm{H}$ NMR spectrum of $2 \mathbf{e}\left(400 \mathrm{MHz}, \mathrm{CDCl}_{3}\right)$

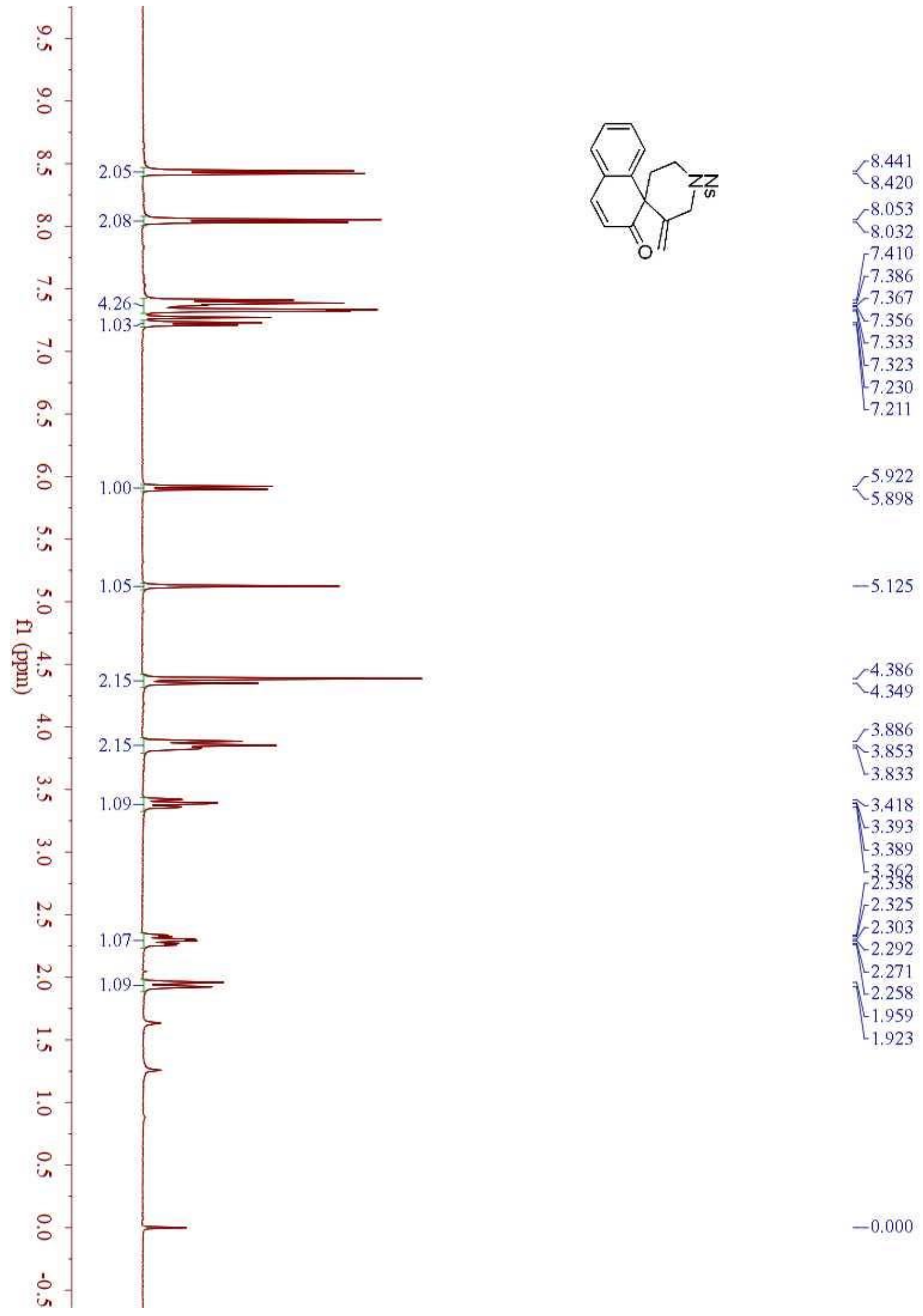


Figure $46{ }^{13} \mathrm{C}$ NMR spectrum of $2 \mathbf{e}\left(100 \mathrm{MHz}, \mathrm{CDCl}_{3}\right)$

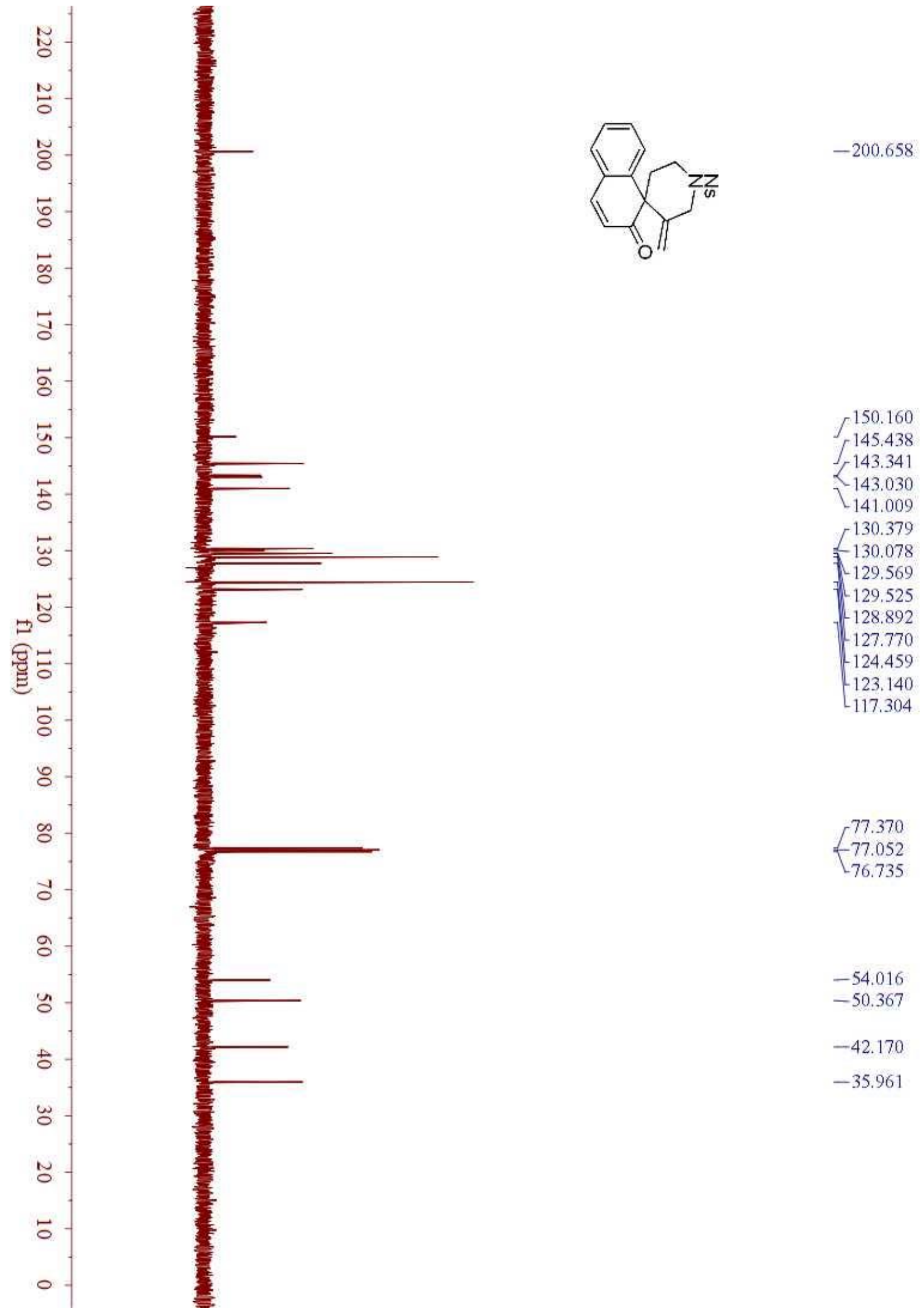


Figure $47{ }^{1} \mathrm{H}$ NMR spectrum of $2 \mathbf{f}\left(400 \mathrm{MHz}, \mathrm{CDCl}_{3}\right)$

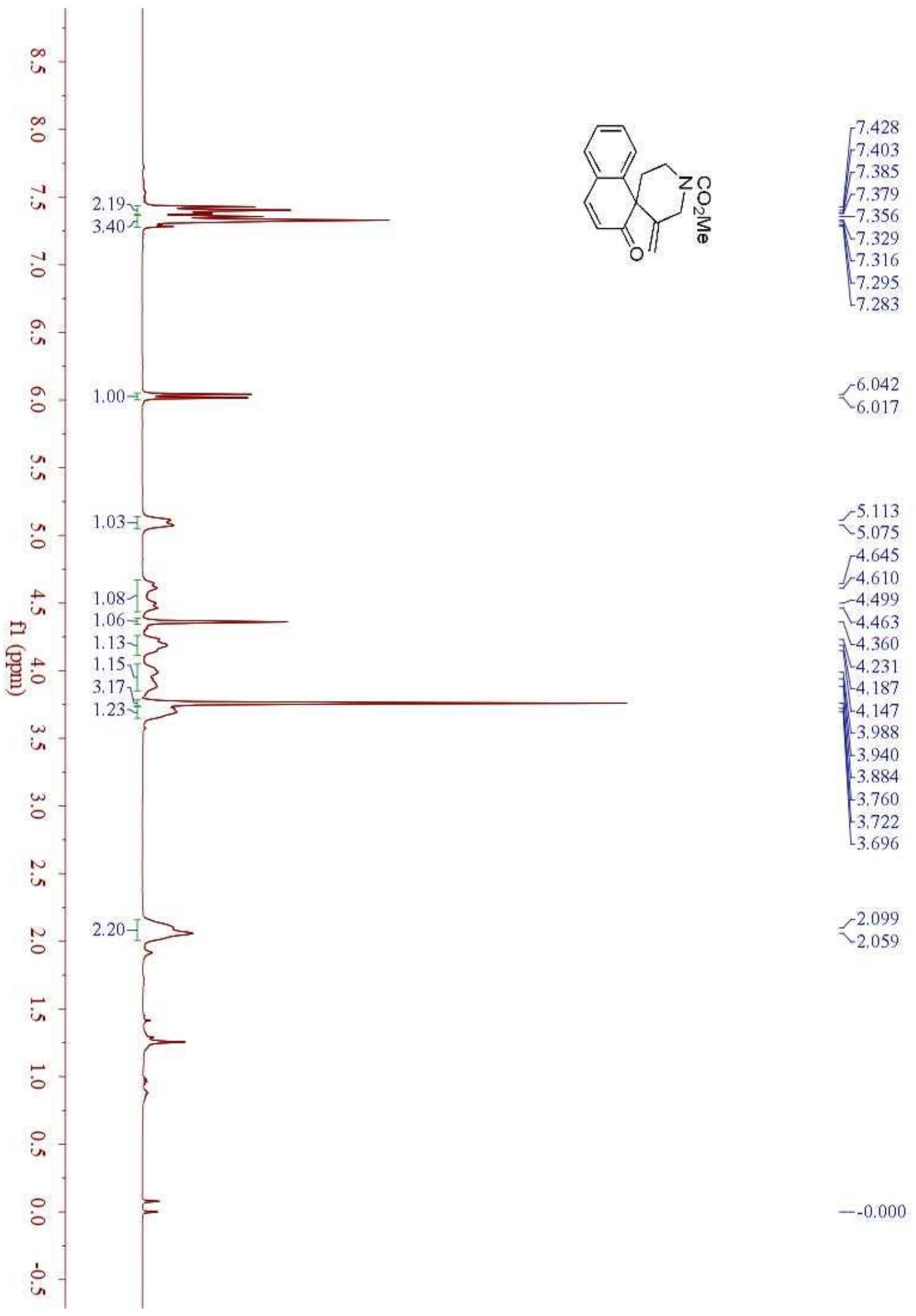


Figure $48{ }^{13} \mathrm{C}$ NMR spectrum of $2 \mathbf{f}\left(100 \mathrm{MHz}, \mathrm{CDCl}_{3}\right)$
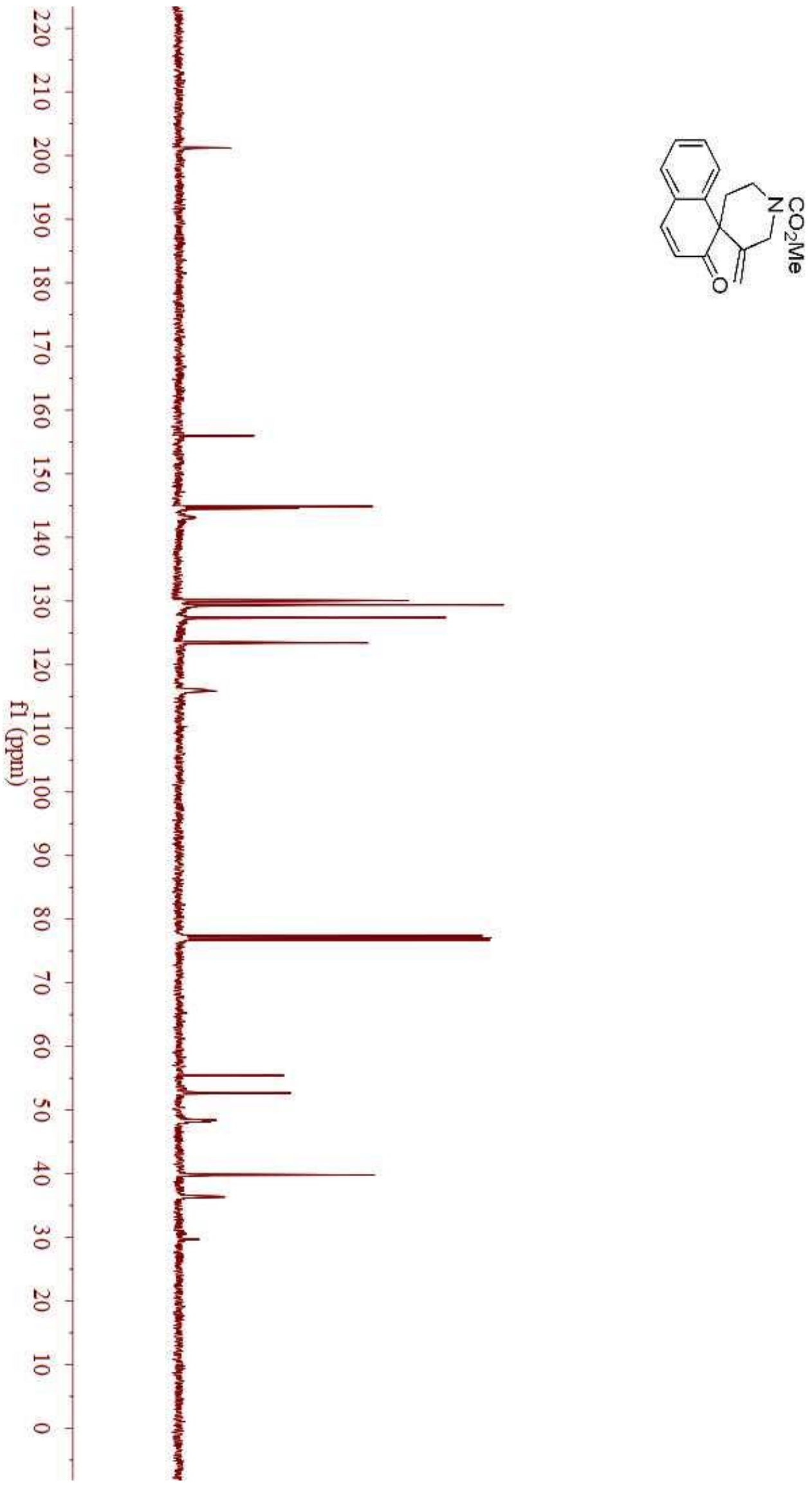
Figure $49{ }^{1} \mathrm{H}$ NMR spectrum of $2 \mathrm{~g}\left(400 \mathrm{MHz}, \mathrm{CDCl}_{3}\right)$

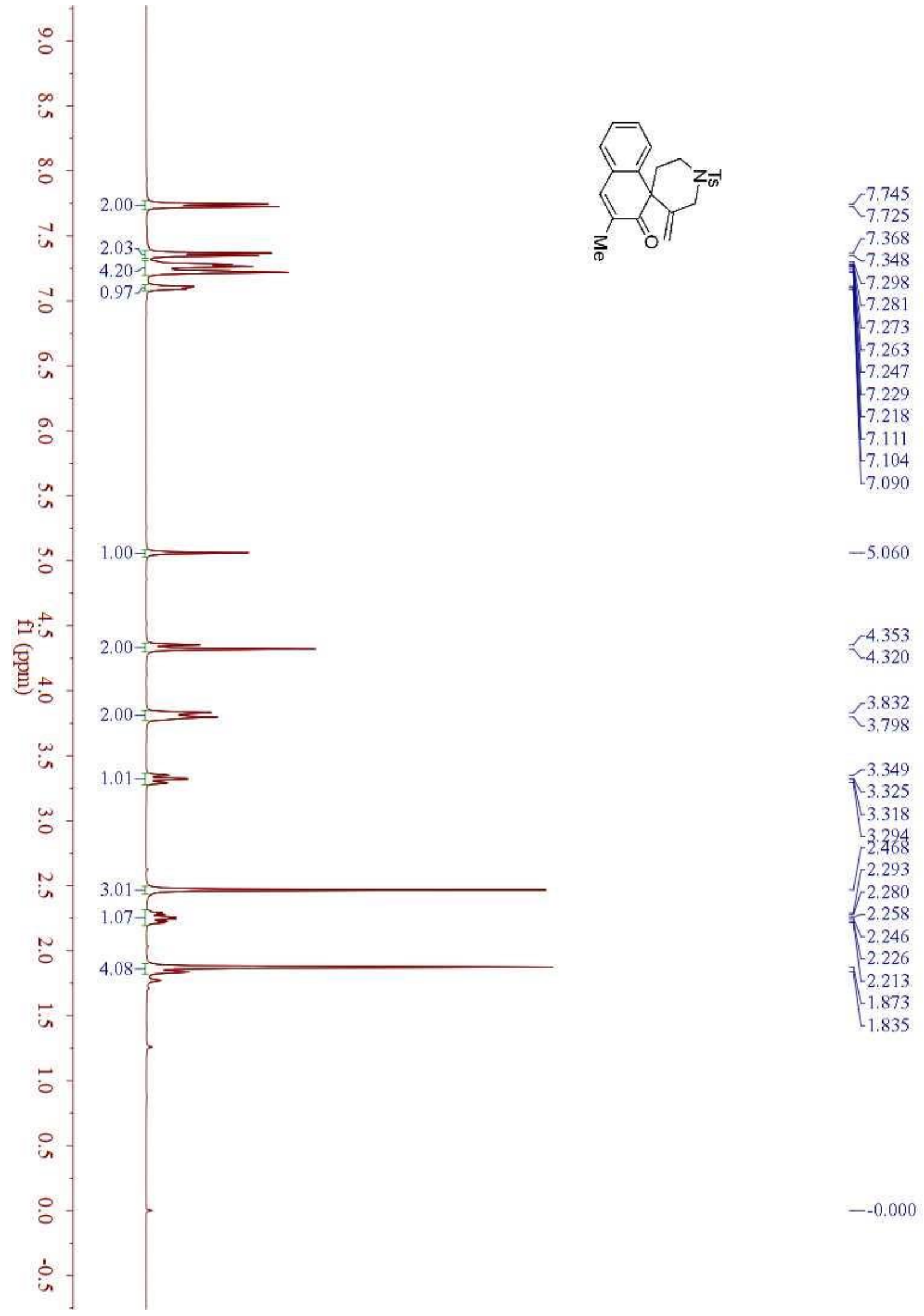


Figure $50{ }^{13} \mathrm{C}$ NMR spectrum of $2 \mathbf{g}\left(100 \mathrm{MHz}, \mathrm{CDCl}_{3}\right)$

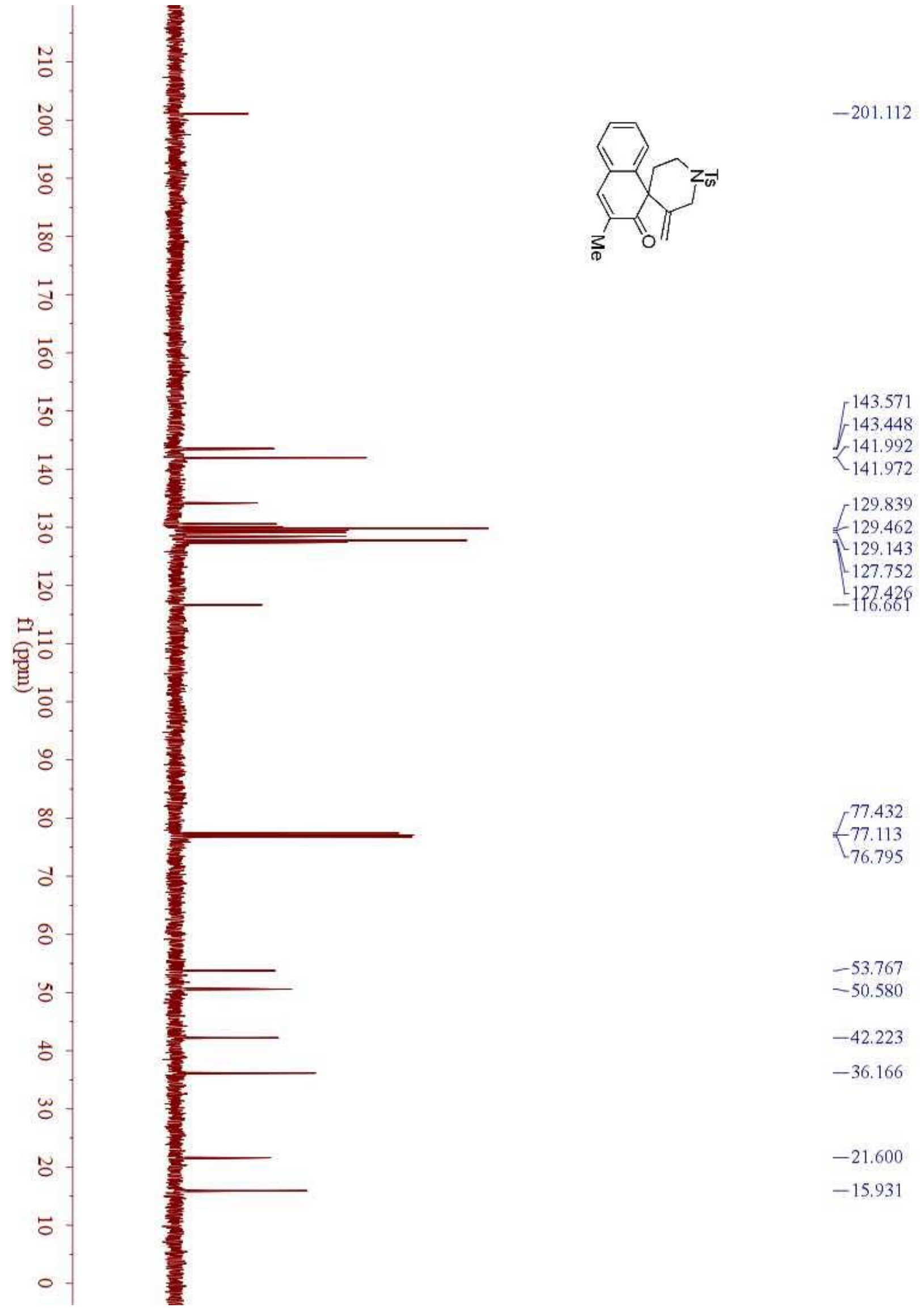


Figure $51{ }^{1} \mathrm{H}$ NMR spectrum of $\mathbf{2 h}\left(400 \mathrm{MHz}, \mathrm{CDCl}_{3}\right)$

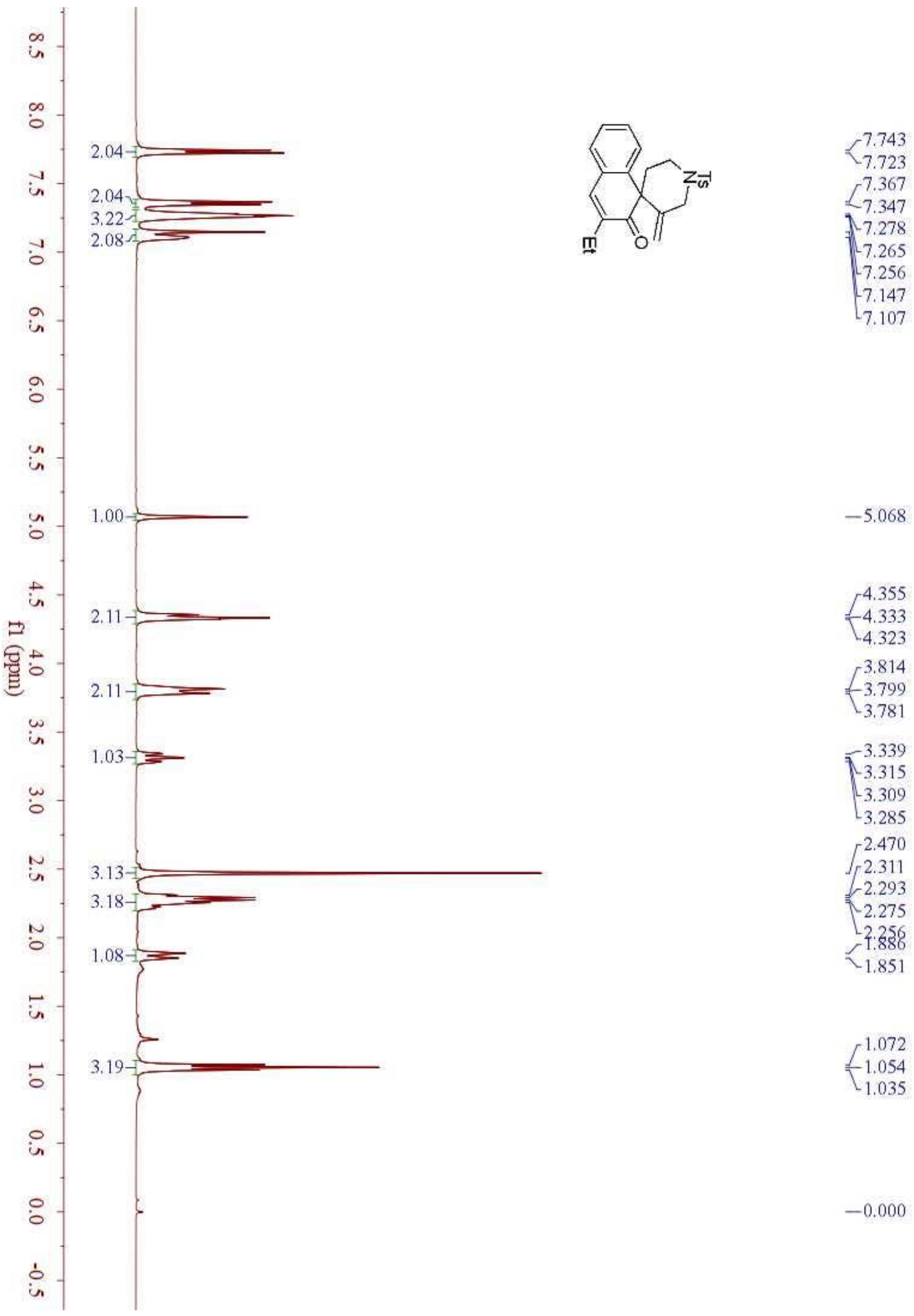


Figure $52{ }^{13} \mathrm{C}$ NMR spectrum of $\mathbf{2 h}\left(100 \mathrm{MHz}, \mathrm{CDCl}_{3}\right)$

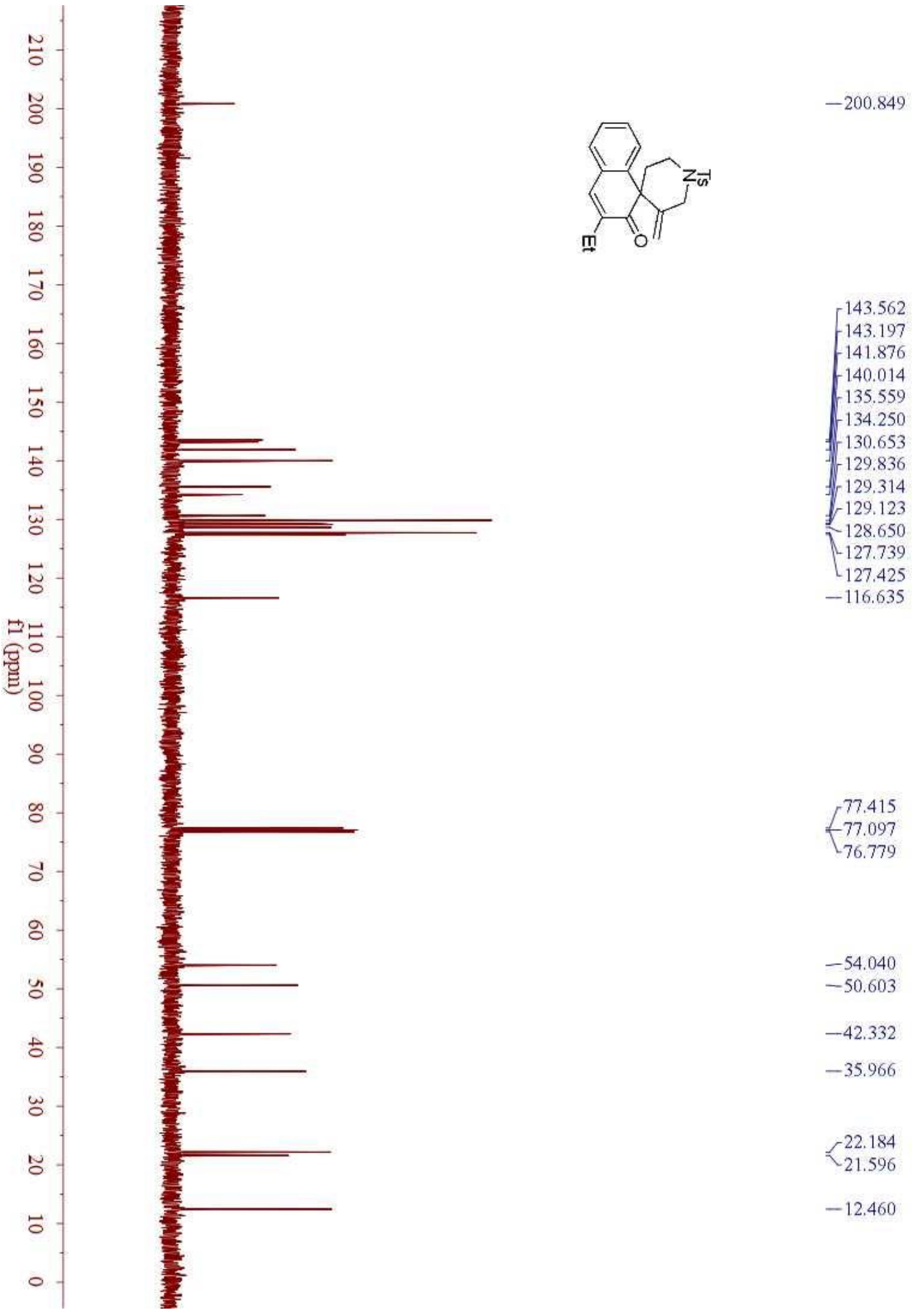


Figure $53{ }^{1} \mathrm{H}$ NMR spectrum of $2 \mathbf{i}\left(400 \mathrm{MHz}, \mathrm{CDCl}_{3}\right)$

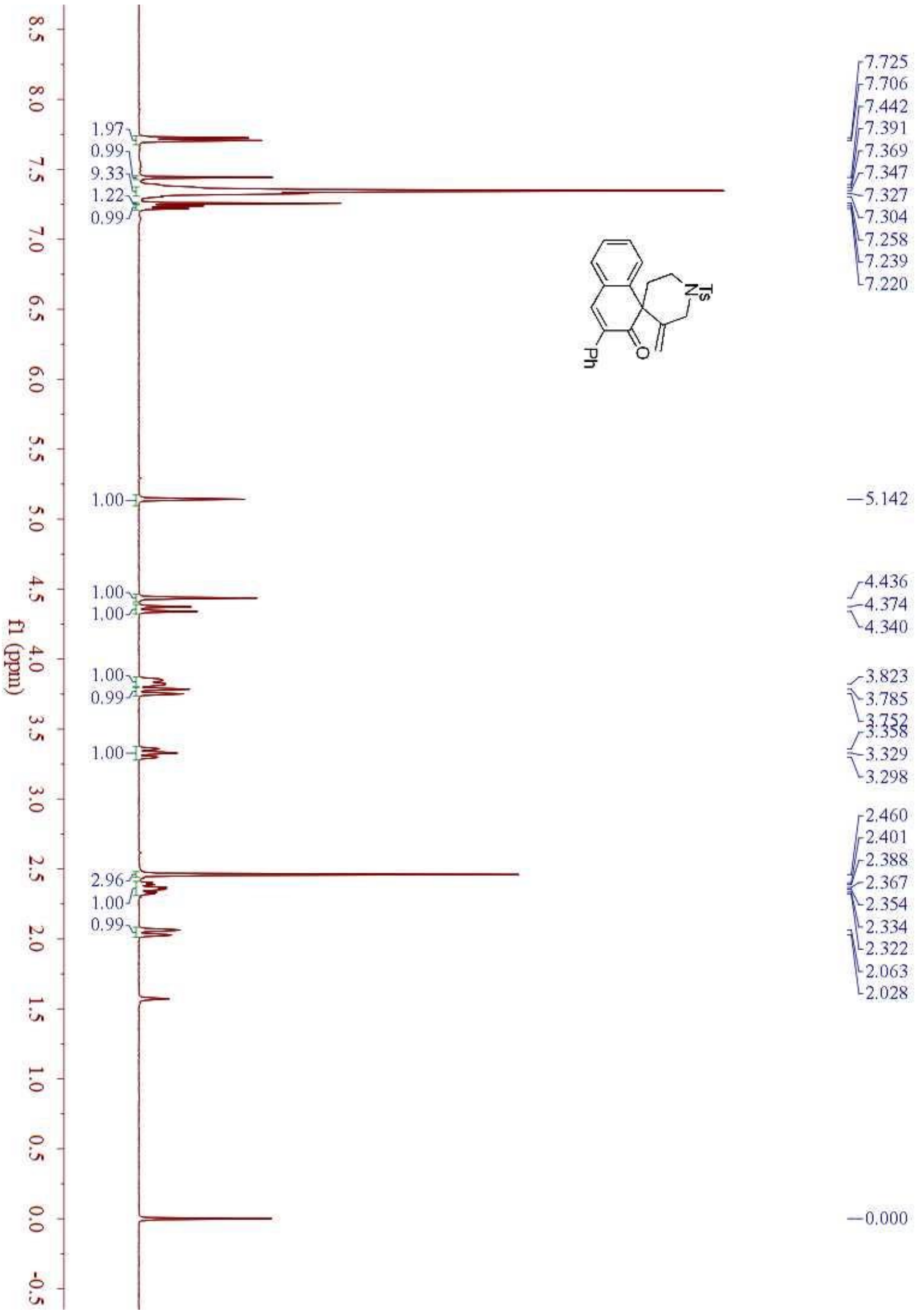


Figure $54{ }^{13} \mathrm{C}$ NMR spectrum of $2 \mathbf{i}\left(100 \mathrm{MHz}, \mathrm{CDCl}_{3}\right)$

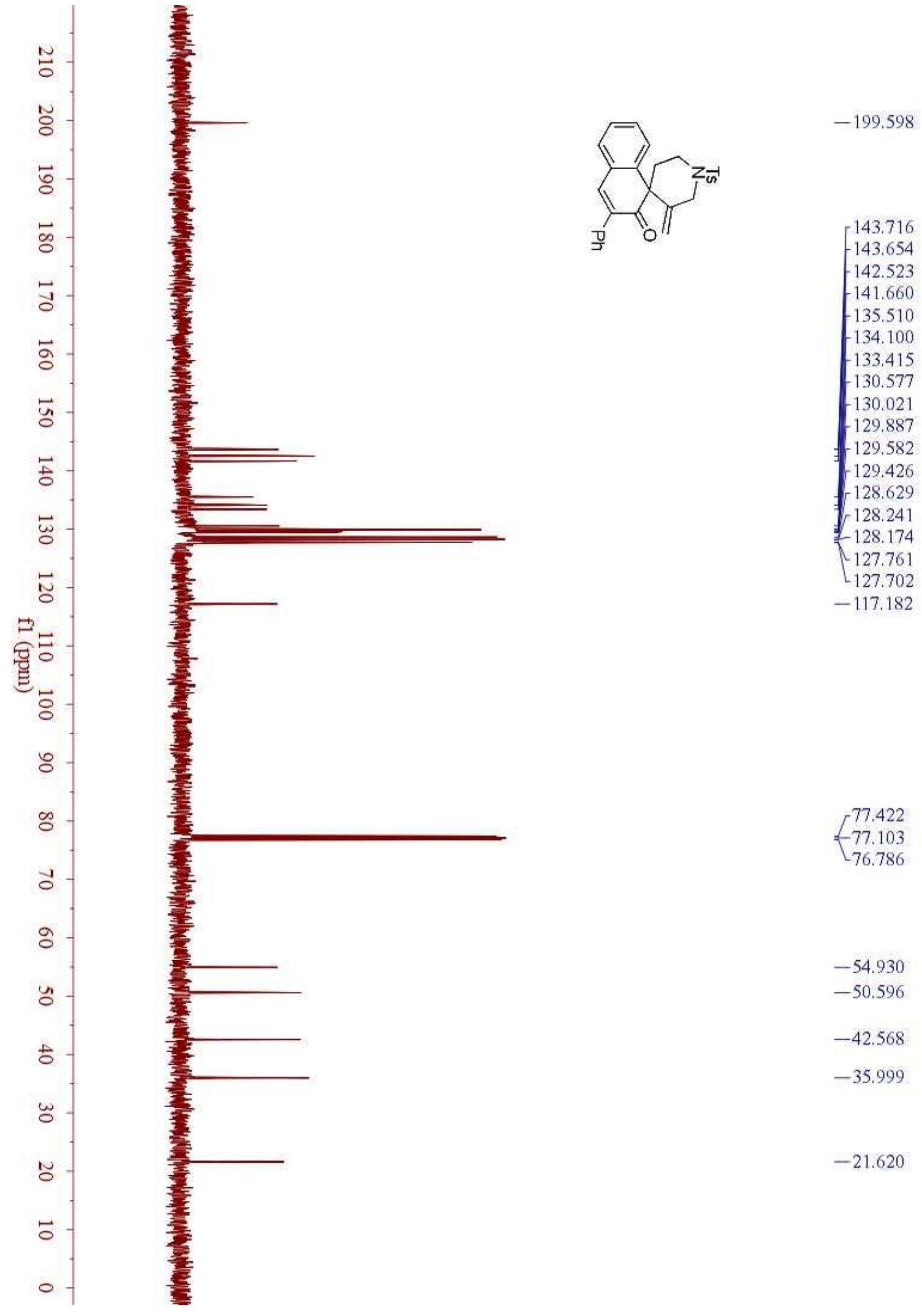


Figure $55{ }^{1} \mathrm{H}$ NMR spectrum of $\mathbf{2 j}\left(400 \mathrm{MHz}, \mathrm{CDCl}_{3}\right)$

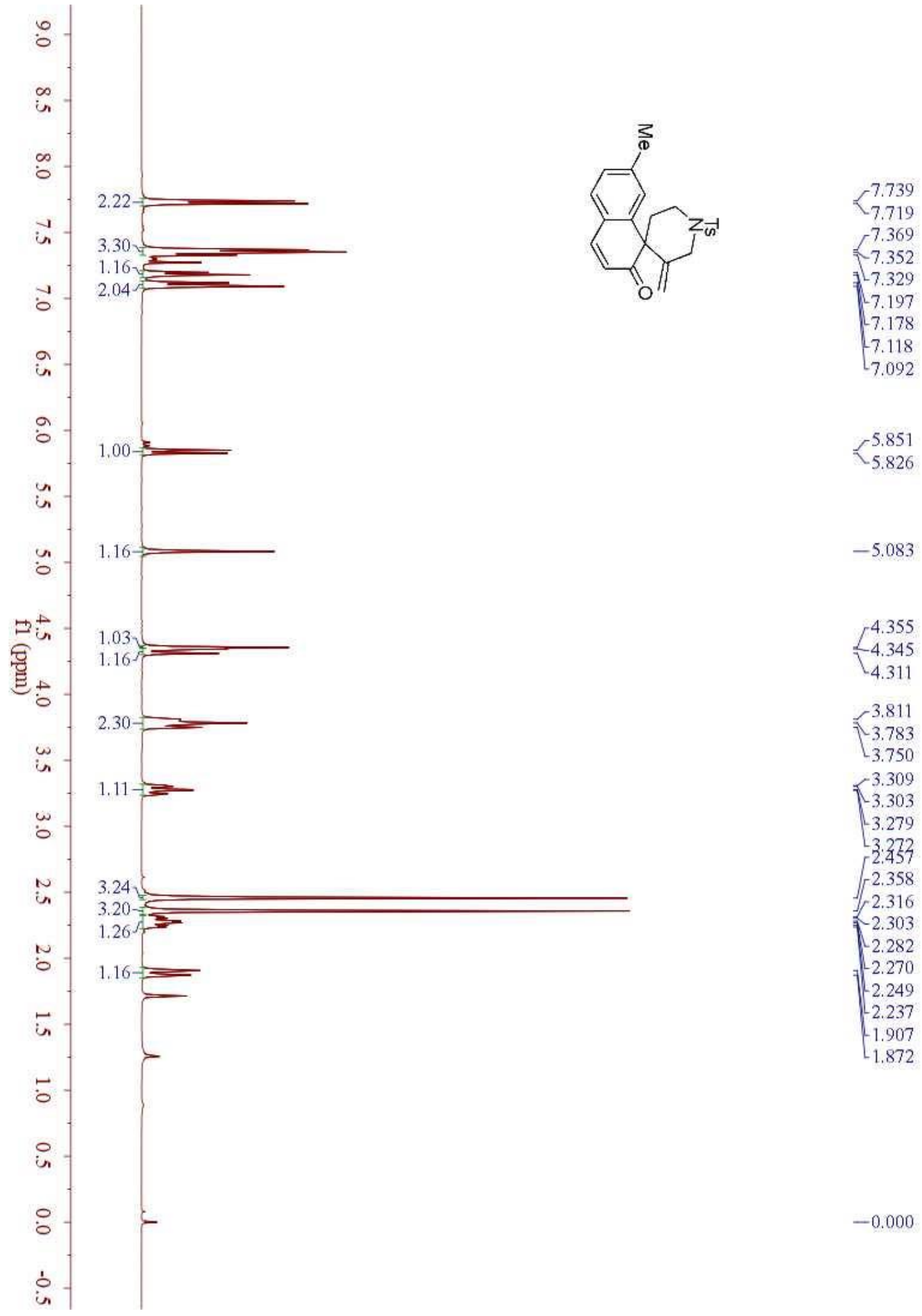


Figure $56{ }^{13} \mathrm{C}$ NMR spectrum of $\mathbf{2 j}\left(100 \mathrm{MHz}, \mathrm{CDCl}_{3}\right)$

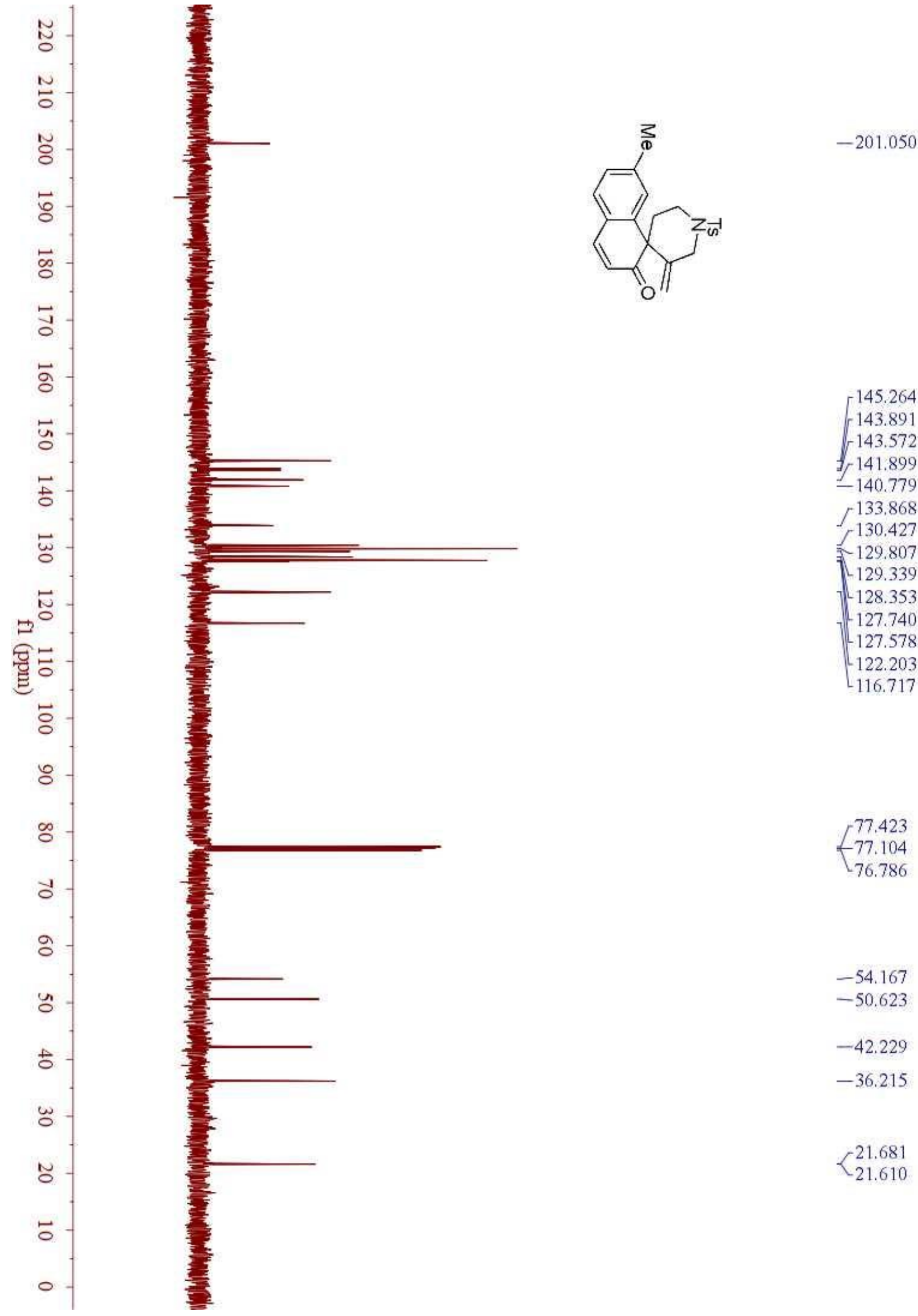


Figure $57{ }^{1} \mathrm{H}$ NMR spectrum of $\mathbf{2 k}\left(400 \mathrm{MHz}, \mathrm{CDCl}_{3}\right)$

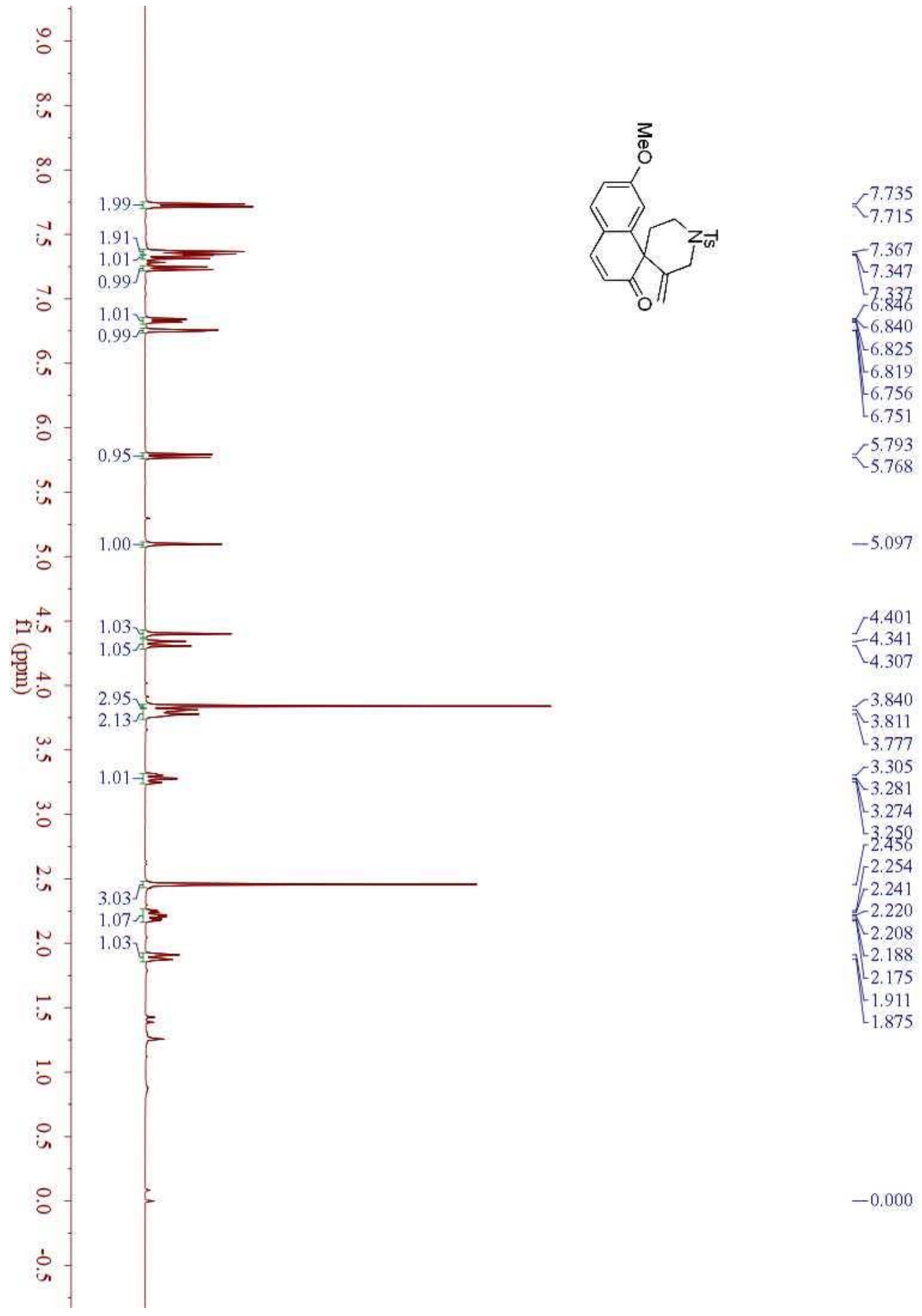


Figure $58{ }^{13} \mathrm{C}$ NMR spectrum of $\mathbf{2 k}\left(100 \mathrm{MHz}, \mathrm{CDCl}_{3}\right)$
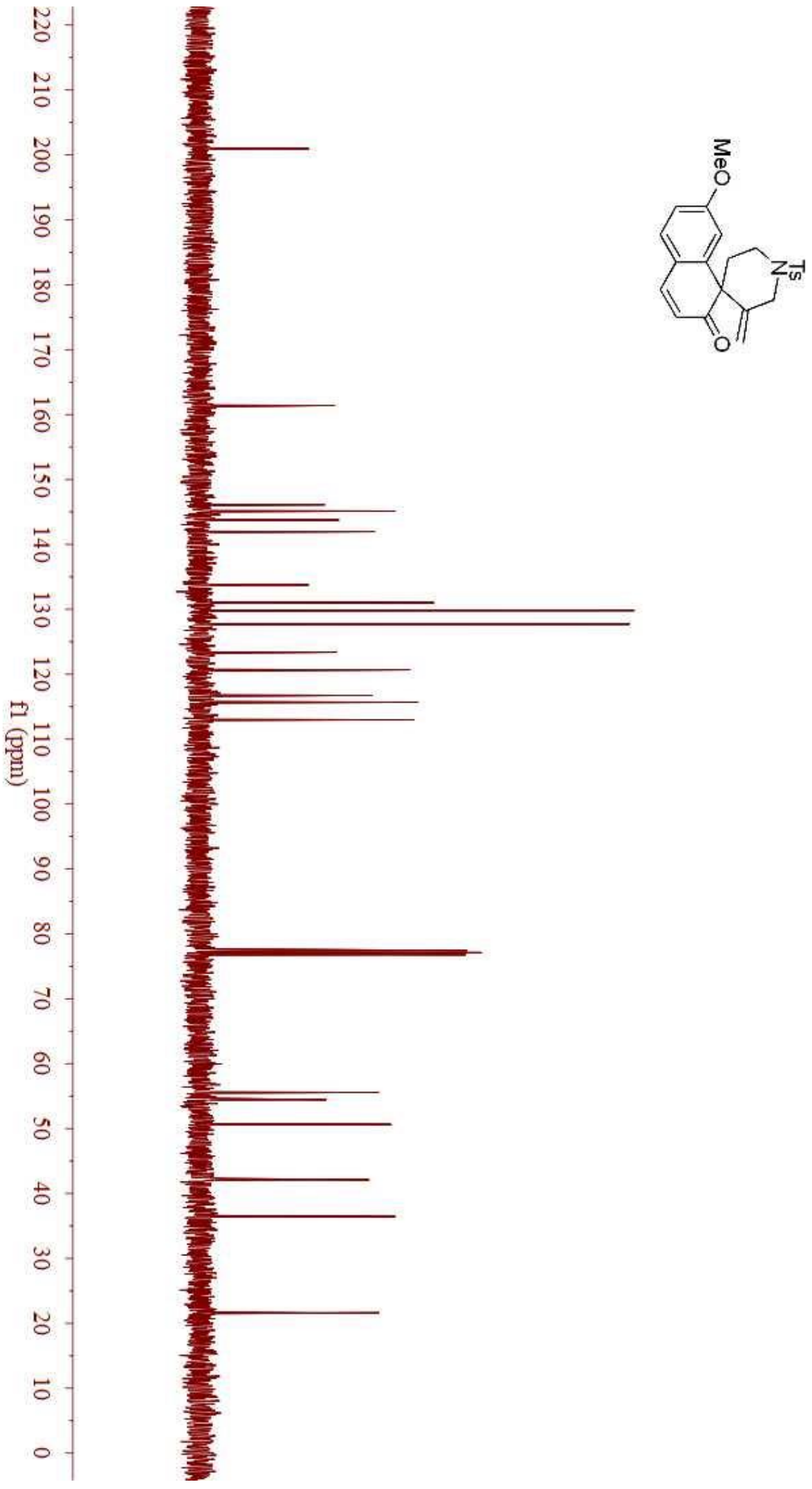

$-200.979$

$-161.405$

$-146.110$

145.142

$-143.744$

$\searrow_{141.963}$

133.761

$-131.004$

$-129.825$

$-127.723$

$-123.387$

$-120.670$

$-116.742$

$-115.679$

$-112.981$

77.424

$-77.106$

$-76.787$

$-55.572$

$-54.450$

$\checkmark 50.639$

$-42.118$

$-36.440$

$-21.596$ 
Figure $59{ }^{1} \mathrm{H}$ NMR spectrum of $2 \mathbf{2 l}\left(400 \mathrm{MHz}, \mathrm{CDCl}_{3}\right)$

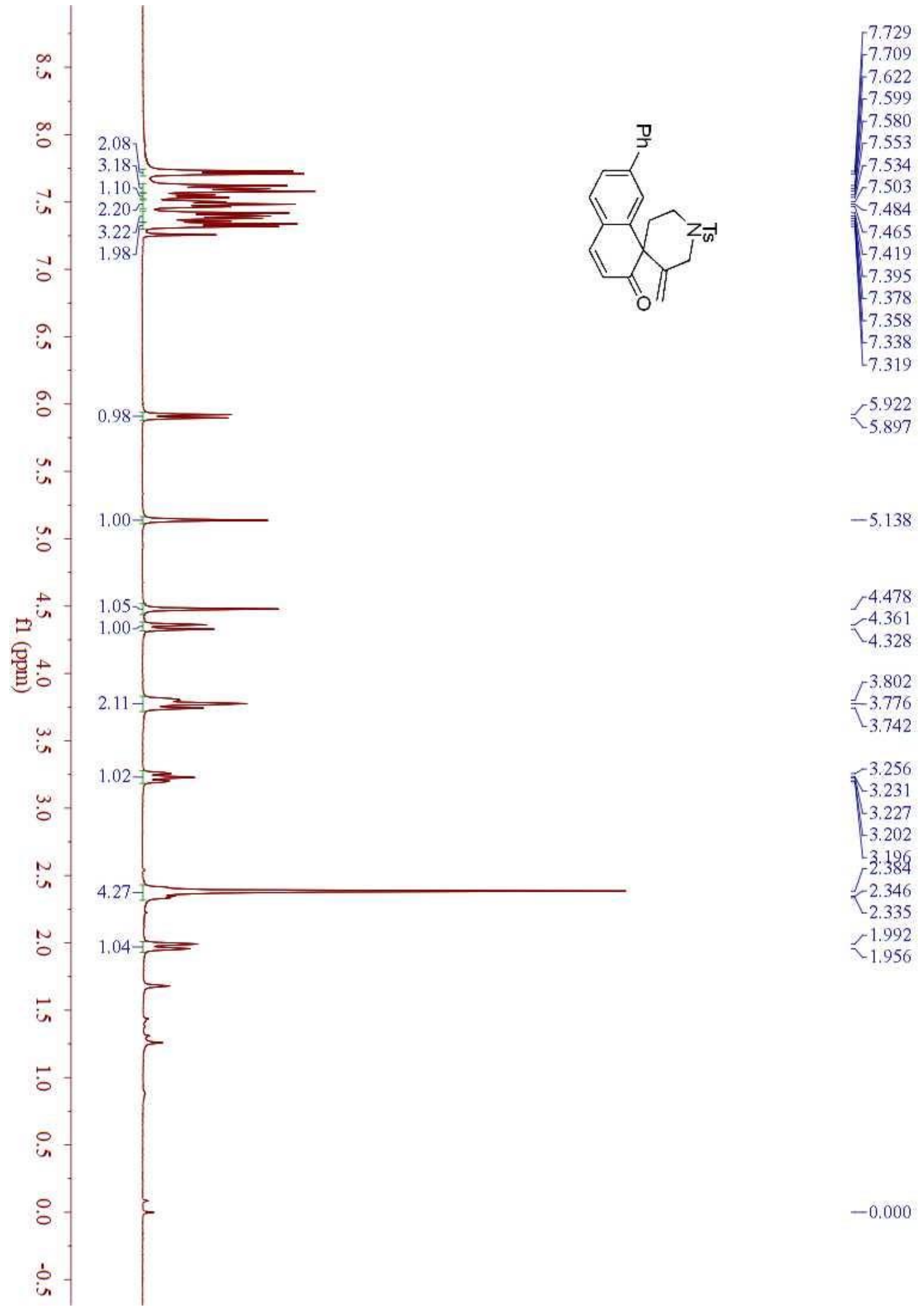


Figure $60{ }^{13} \mathrm{C}$ NMR spectrum of $21\left(100 \mathrm{MHz}, \mathrm{CDCl}_{3}\right)$
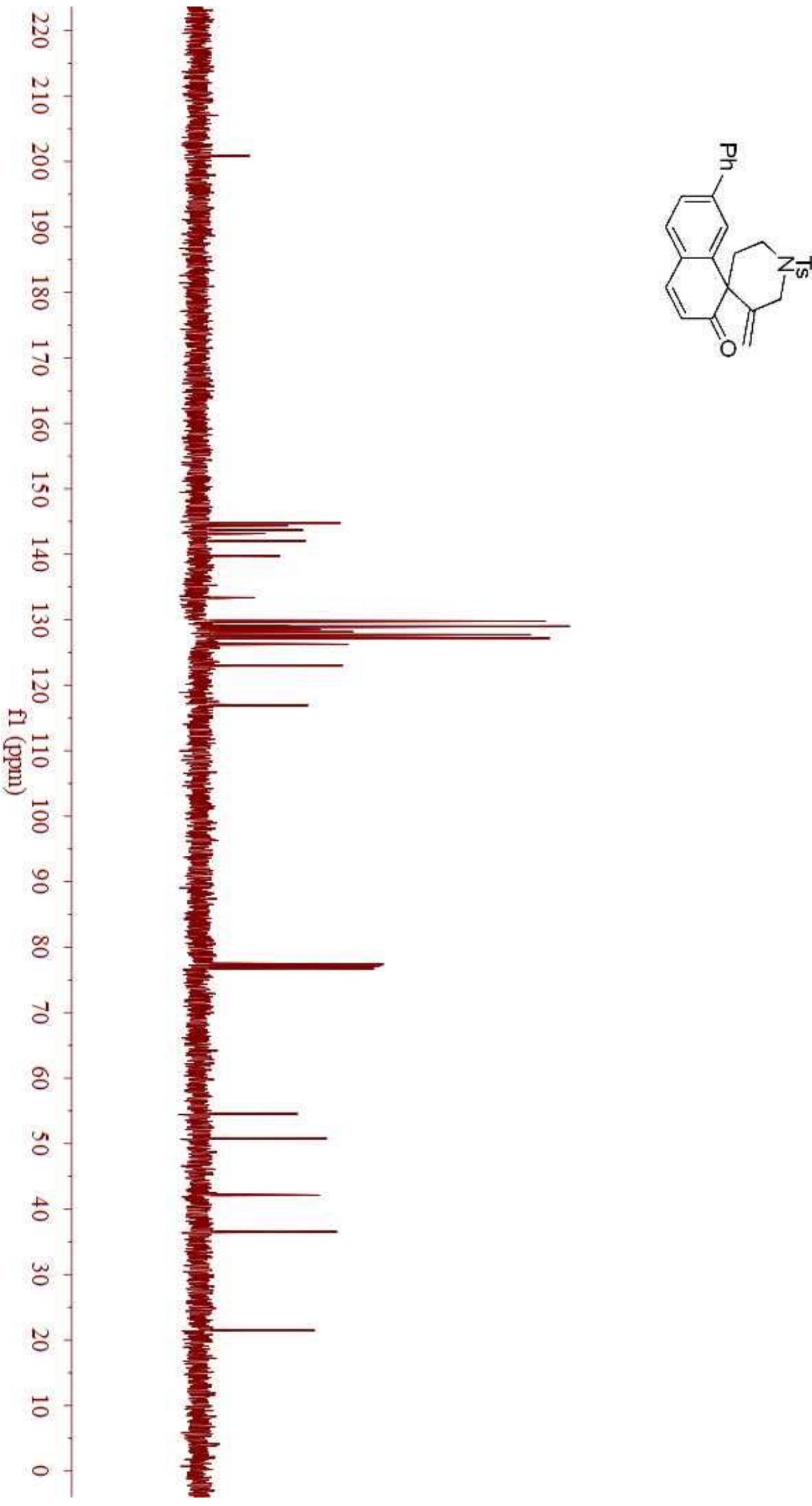

$-200.856$

144.795

$-144.396$

$-143.698$

$-143.175$

142.017

139.751

133.379

$-129.868$

$-129.797$

$-129.117$

$-129.000$

$-128.557$

$-128.168$

$-127.729$

$-127.190$

$-126.257$

$-123.026$

$-116.924$

77.384

$-77.059$

$-76.747$

$-54.552$

$-50.766$

$-42.121$

$-36.553$

$-21.517$ 
Figure $61{ }^{1} \mathrm{H}$ NMR spectrum of $\mathbf{2 m}\left(400 \mathrm{MHz}, \mathrm{CDCl}_{3}\right)$

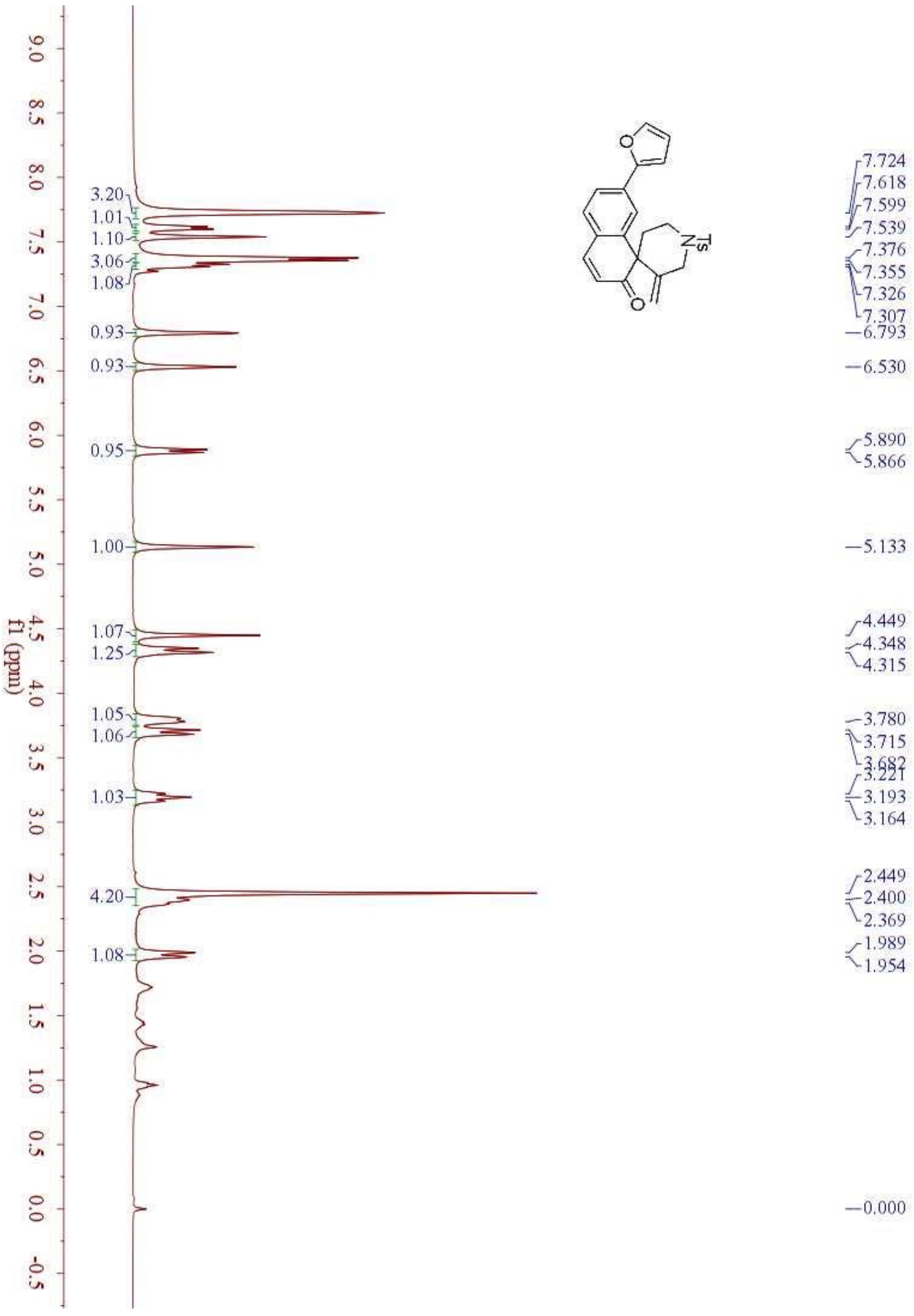


Figure $62{ }^{13} \mathrm{C}$ NMR spectrum of $\mathbf{2 m}\left(100 \mathrm{MHz}, \mathrm{CDCl}_{3}\right)$
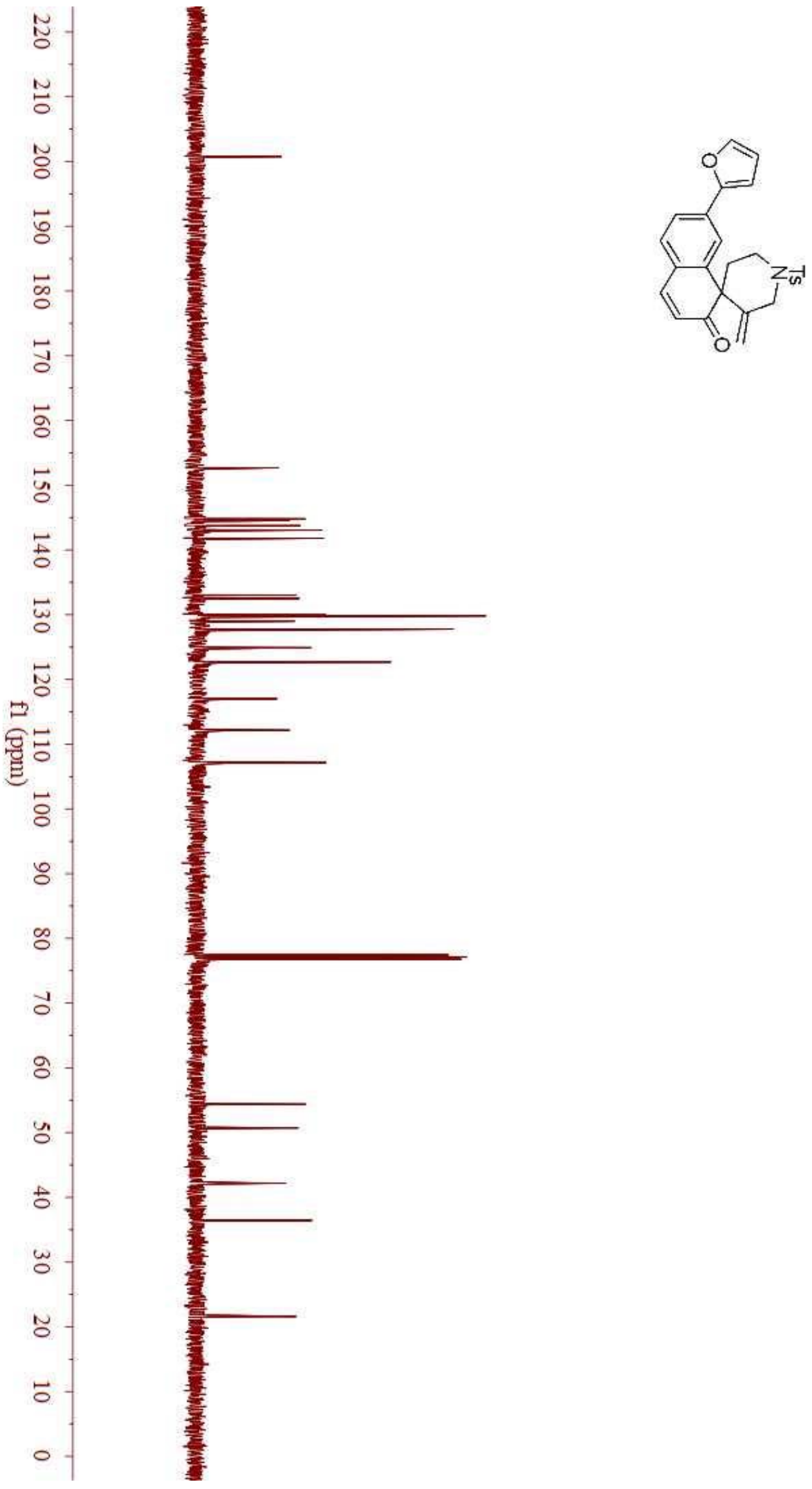
Figure $63{ }^{1} \mathrm{H}$ NMR spectrum of 2 n $\left(400 \mathrm{MHz}, \mathrm{CDCl}_{3}\right)$

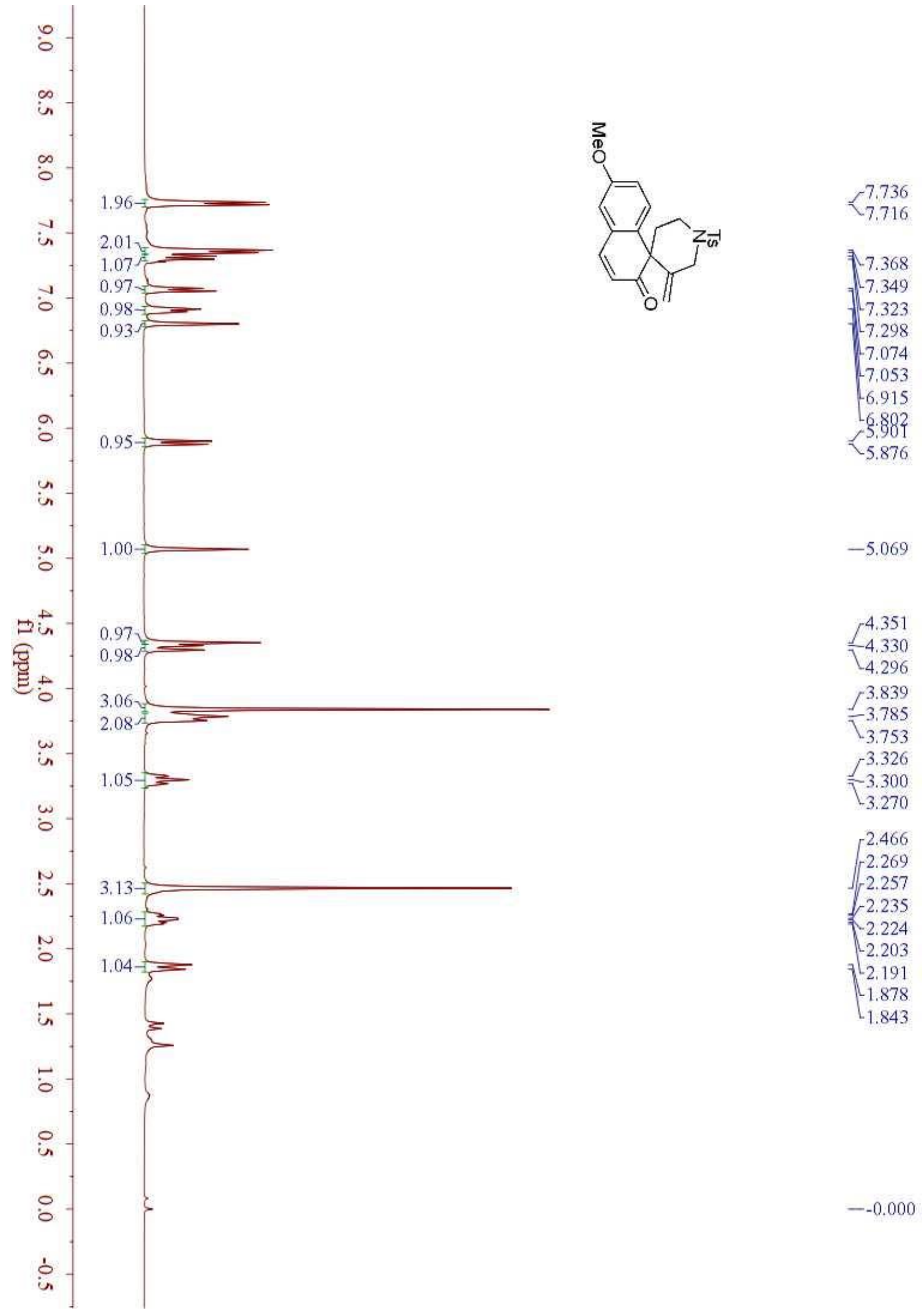


Figure $64{ }^{13} \mathrm{C}$ NMR spectrum of 2 n $\left(100 \mathrm{MHz}, \mathrm{CDCl}_{3}\right)$
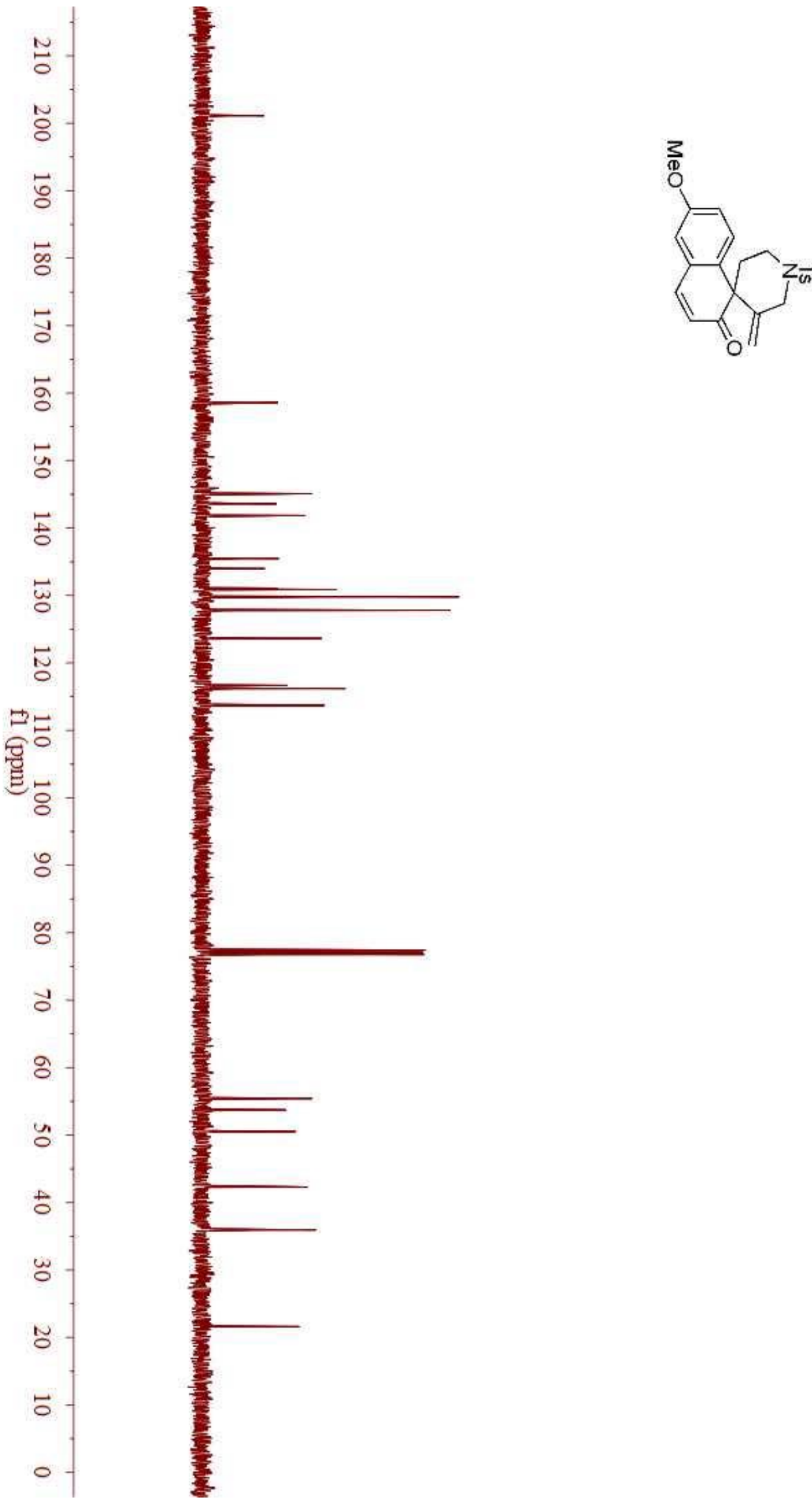

$-158.576$

145.086

143.596

141.859

135.479

133.965

$-131.086$

$-130.864$

$-129.793$

$-127.790$

$-123.613$

116.669

$-116.198$

113.672

77.416

$-77.098$

$-76.780$

$-55.431$

$-53.715$

$\sim 50.539$

$-42.297$

$-35.938$

$-21.596$ 
Figure $65{ }^{1} \mathrm{H}$ NMR spectrum of $20\left(400 \mathrm{MHz}, \mathrm{CDCl}_{3}\right)$

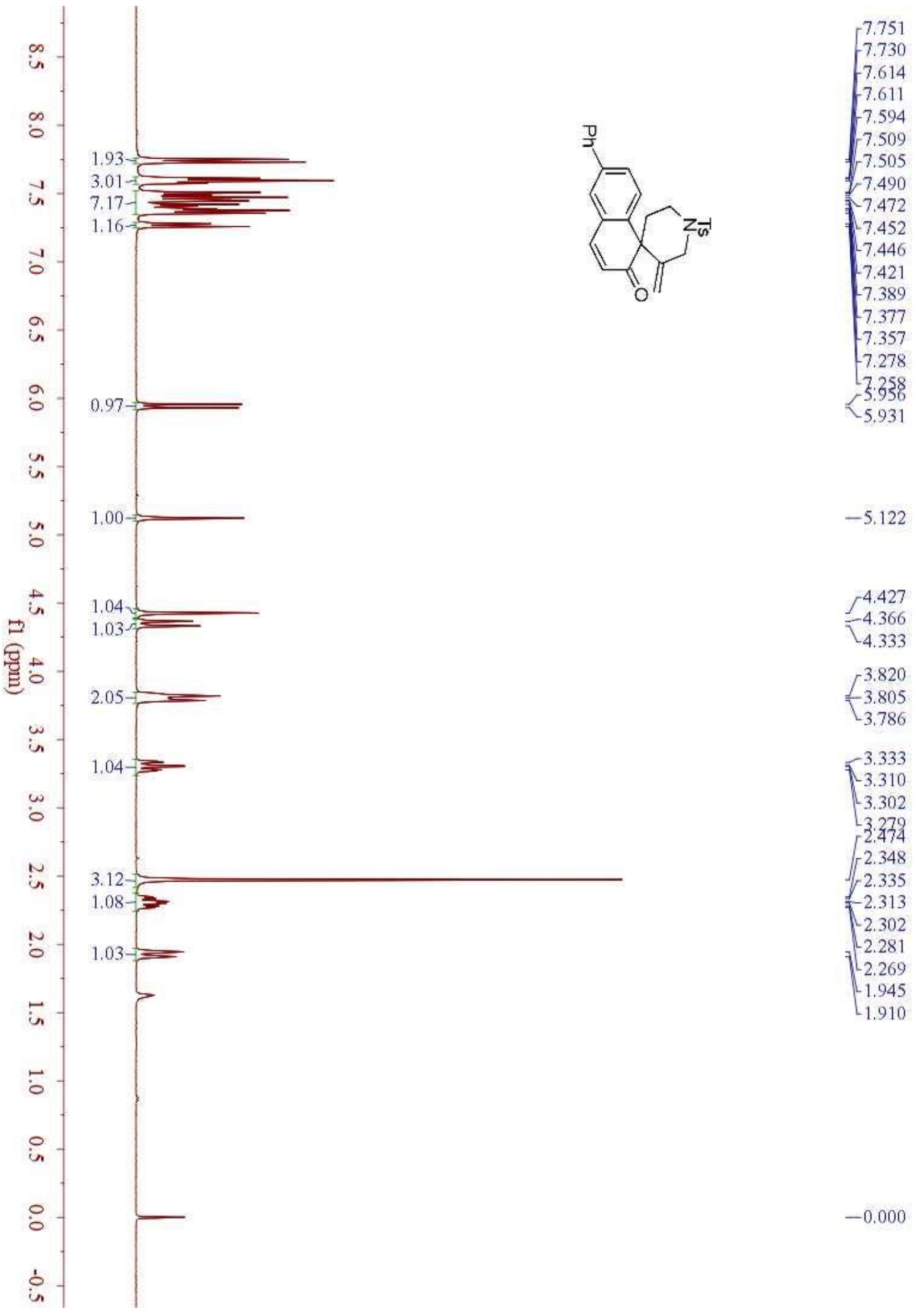


Figure $66{ }^{13} \mathrm{C}$ NMR spectrum of $20\left(100 \mathrm{MHz}, \mathrm{CDCl}_{3}\right)$
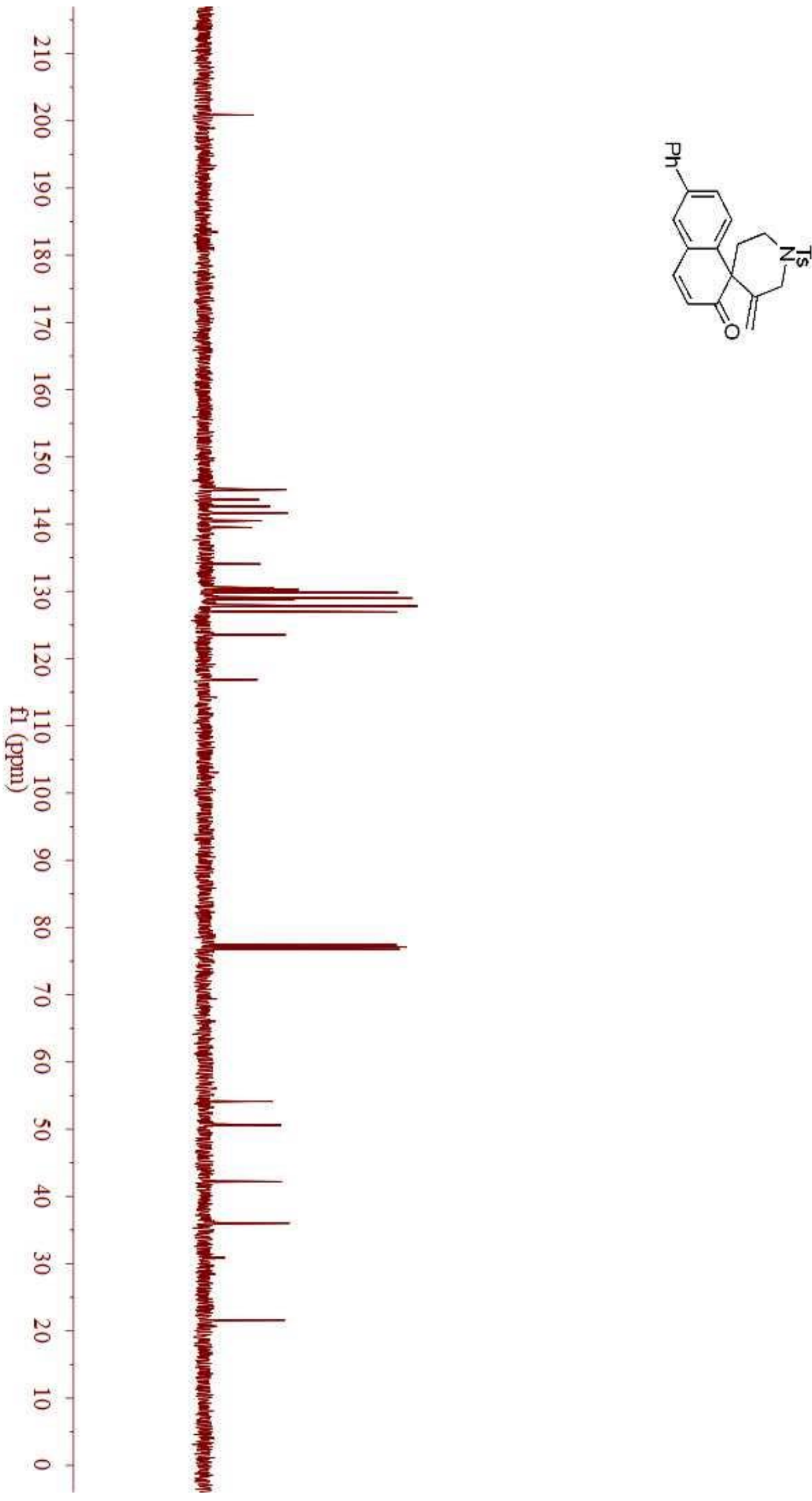

$\left[\begin{array}{l}145.144 \\ -143.625 \\ -142.617 \\ -141.676 \\ -140.515 \\ -139.501 \\ -134.064 \\ -130.522 \\ -130.253 \\ -129.822 \\ 128.980 \\ -128.793 \\ -127.899 \\ -127.816 \\ -126.927 \\ -123.563 \\ 116.881\end{array}\right.$

77.413

$-77.094$

76.776

$-54.136$

$-50.600$

$-42.200$

$-36.036$

$-21.608$ 
Figure $67{ }^{1} \mathrm{H}$ NMR spectrum of $\mathbf{2 p}\left(400 \mathrm{MHz}, \mathrm{CDCl}_{3}\right)$

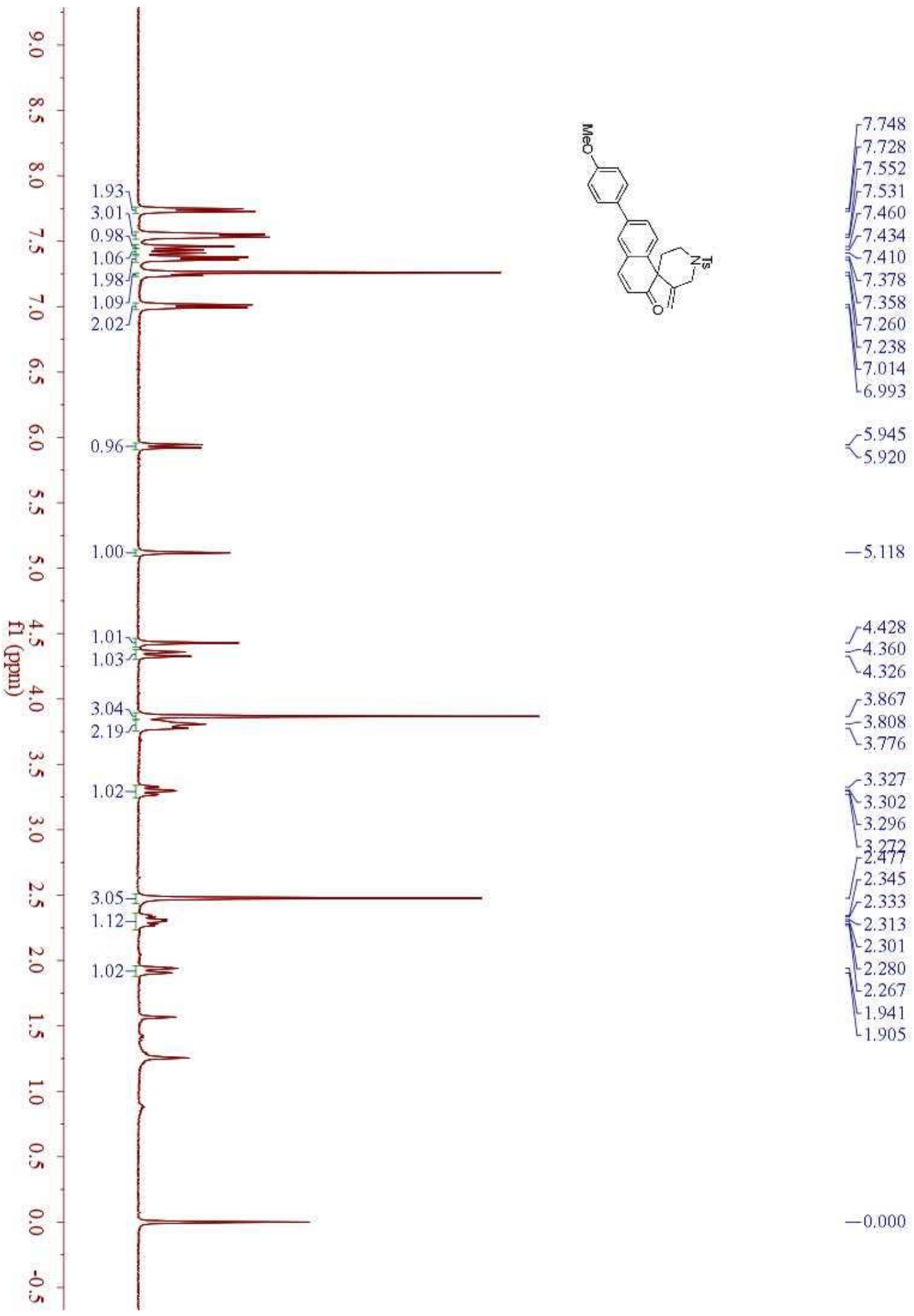


Figure $68{ }^{13} \mathrm{C}$ NMR spectrum of $2 \mathbf{p}\left(100 \mathrm{MHz}, \mathrm{CDCl}_{3}\right)$

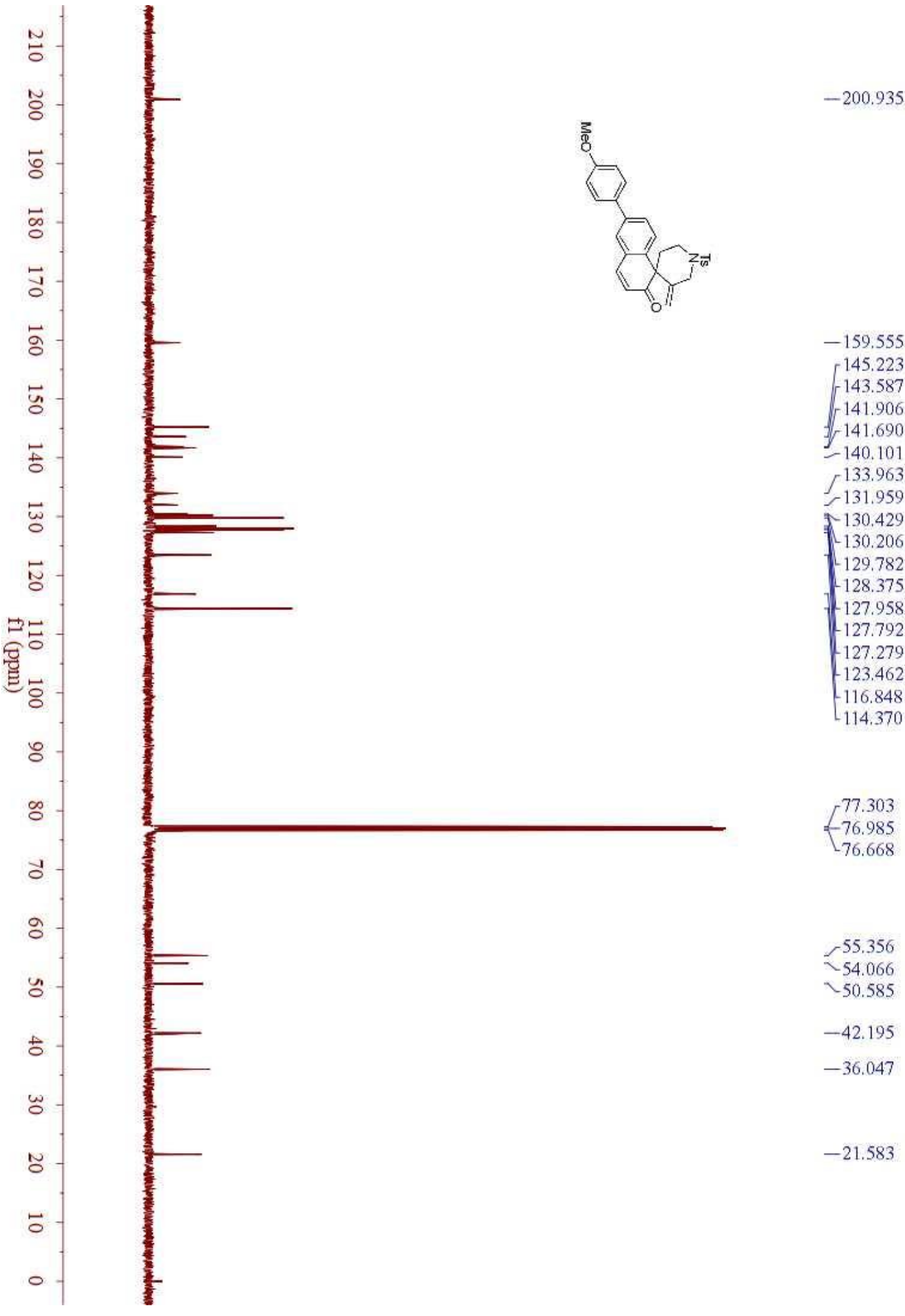


Figure $69{ }^{1} \mathrm{H}$ NMR spectrum of $\mathbf{2 q}\left(400 \mathrm{MHz}, \mathrm{CDCl}_{3}\right)$

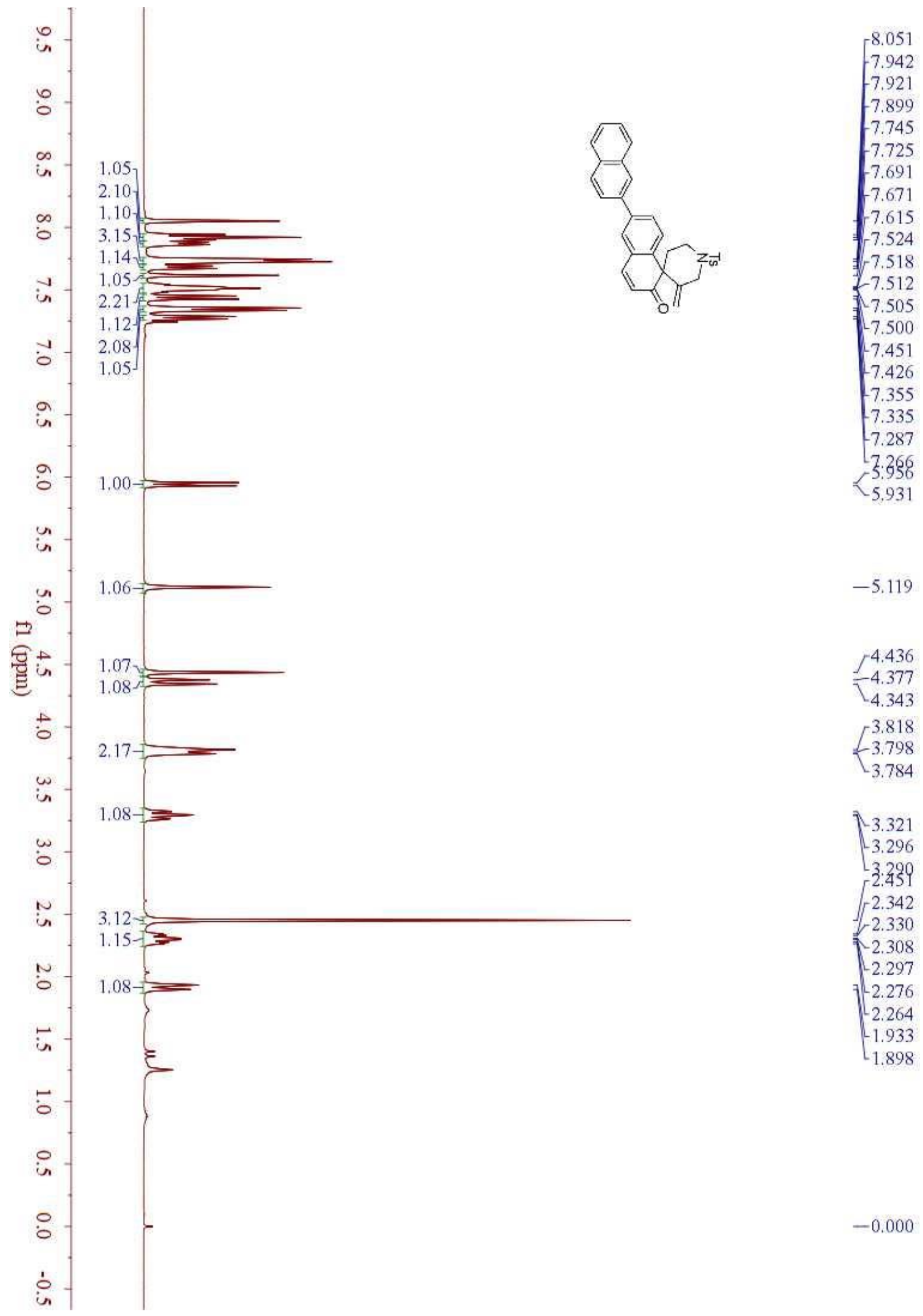


Figure $70{ }^{13} \mathrm{C} \mathrm{NMR}$ spectrum of $\mathbf{2 q}\left(100 \mathrm{MHz}, \mathrm{CDCl}_{3}\right)$
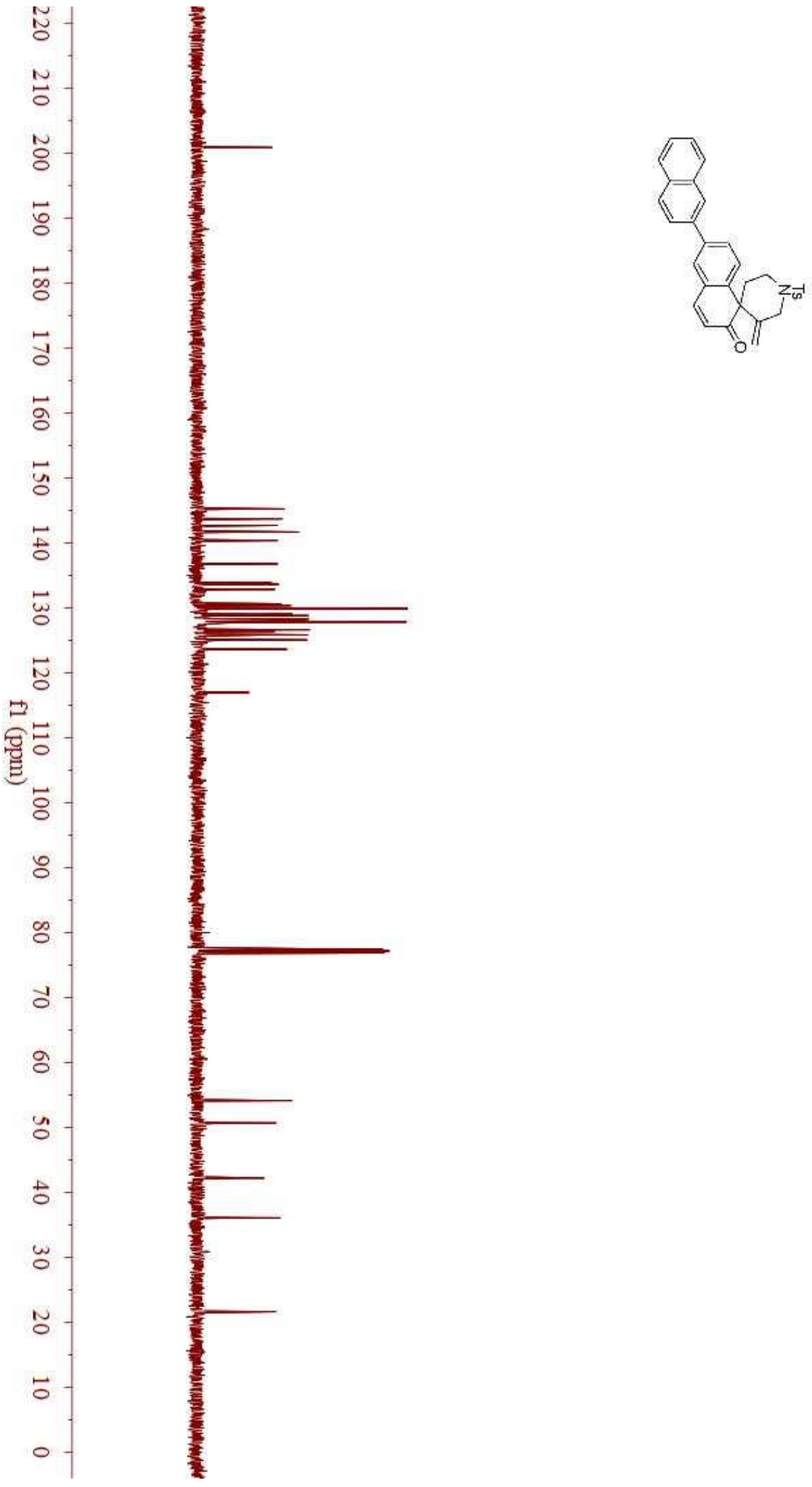

$-200.842$

145.213

$-143.688$

$-142.673$

$-141.668$

$-140.355$

$-136.776$

$-133.872$

$-133.605$

$-132.839$

$-130.621$

$-130.342$

f 129.863

$-129.023$

128.783

$-128.266$

$-128.070$

$-127.834$

$-127.736$

$-126.608$

$-126.369$

$-125.837$

$-125.033$

$-123.601$

$-116.975$

77.499

$-77.181$

$-76.862$

-54.135
-50.672

$-42.233$

$-36.079$

$-21.650$ 
Figure $71{ }^{1} \mathrm{H}$ NMR spectrum of $\mathbf{2 r}\left(400 \mathrm{MHz}, \mathrm{CDCl}_{3}\right)$

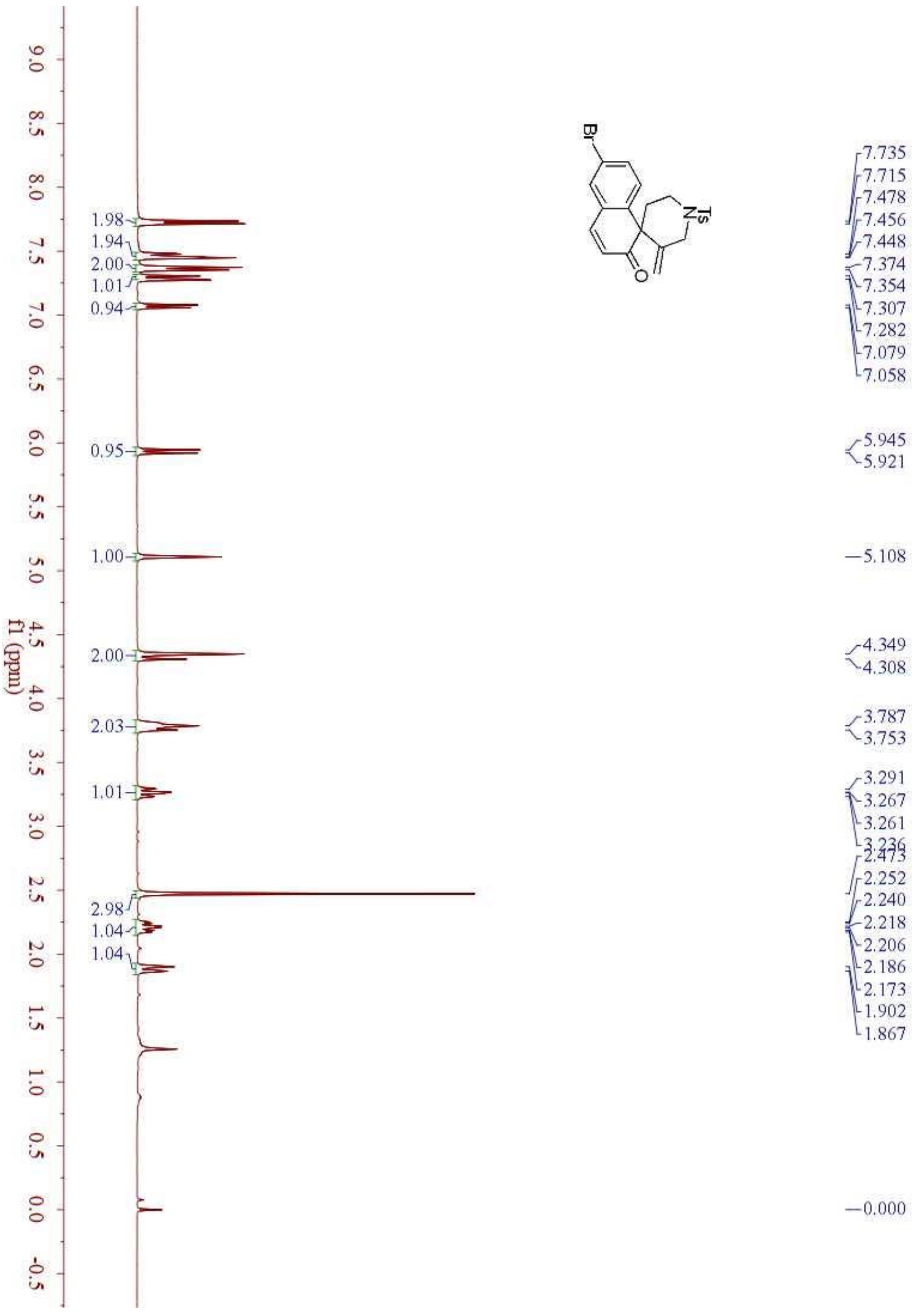


Figure $72{ }^{13} \mathrm{C}$ NMR spectrum of $2 \mathbf{r}\left(100 \mathrm{MHz}, \mathrm{CDCl}_{3}\right)$
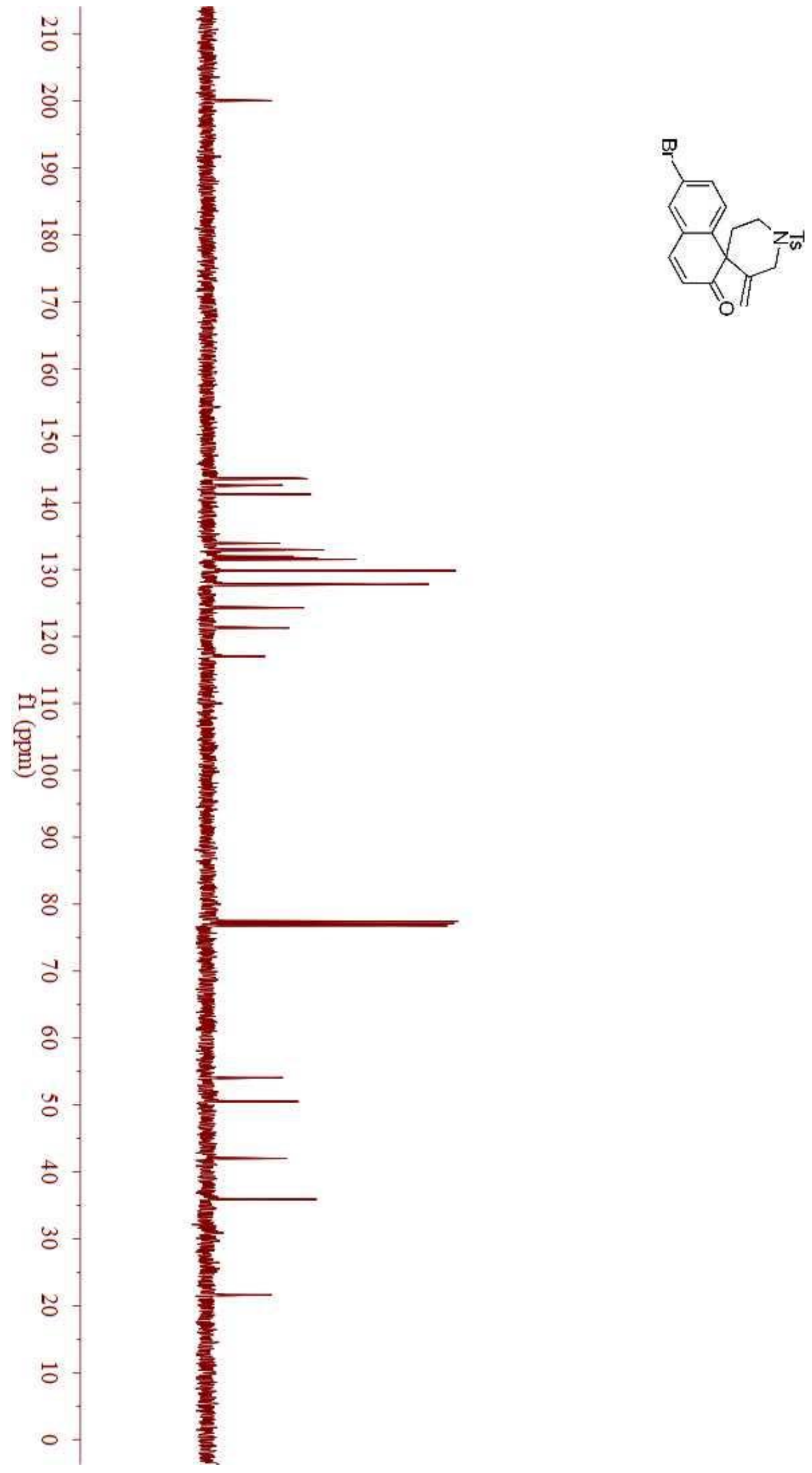
Figure $73{ }^{1} \mathrm{H}$ NMR spectrum of $\mathbf{3 a}\left(400 \mathrm{MHz}, \mathrm{CDCl}_{3}\right)$

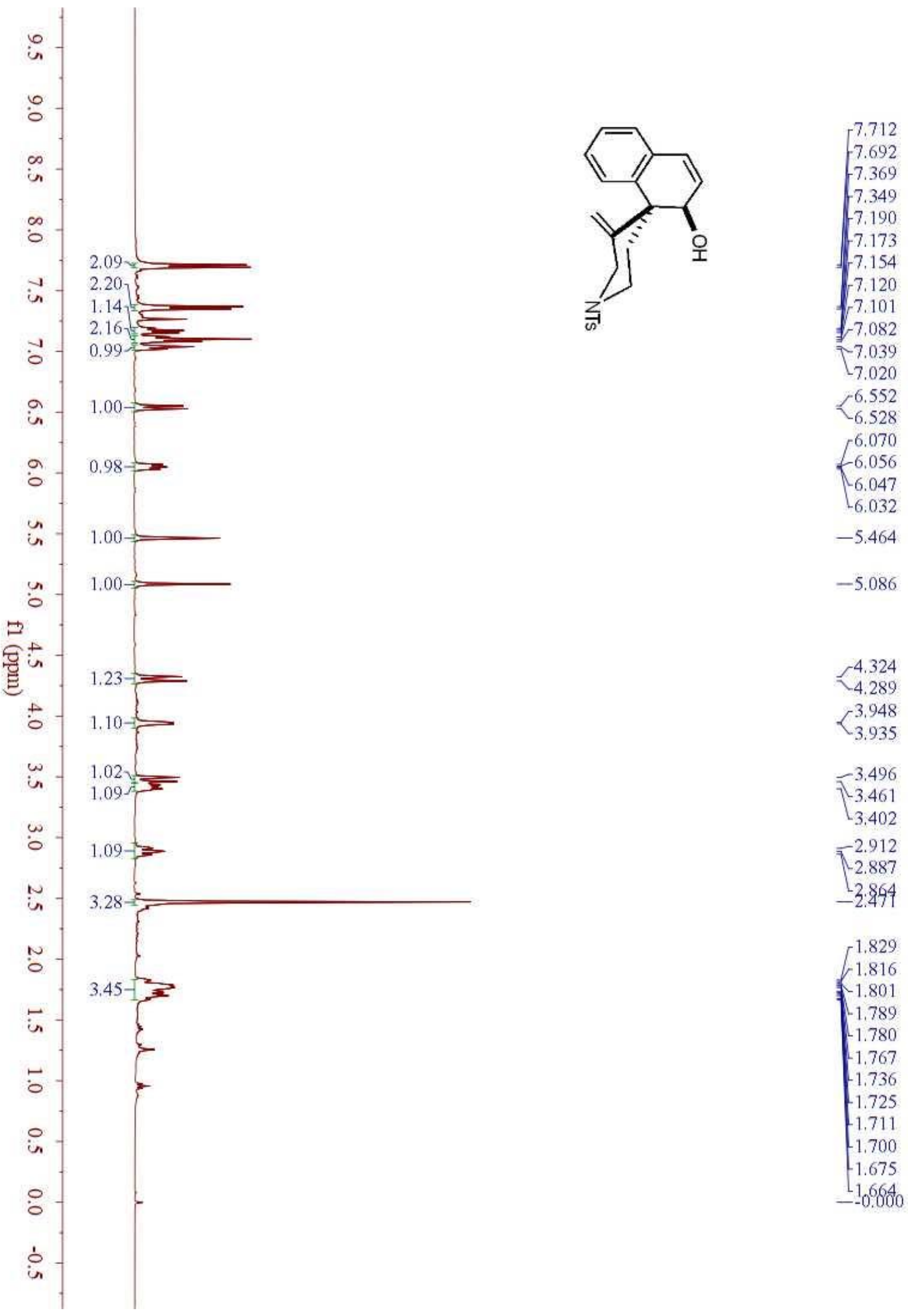


Figure $74{ }^{13} \mathrm{C}$ NMR spectrum of $\mathbf{3 a}\left(100 \mathrm{MHz}, \mathrm{CDCl}_{3}\right)$
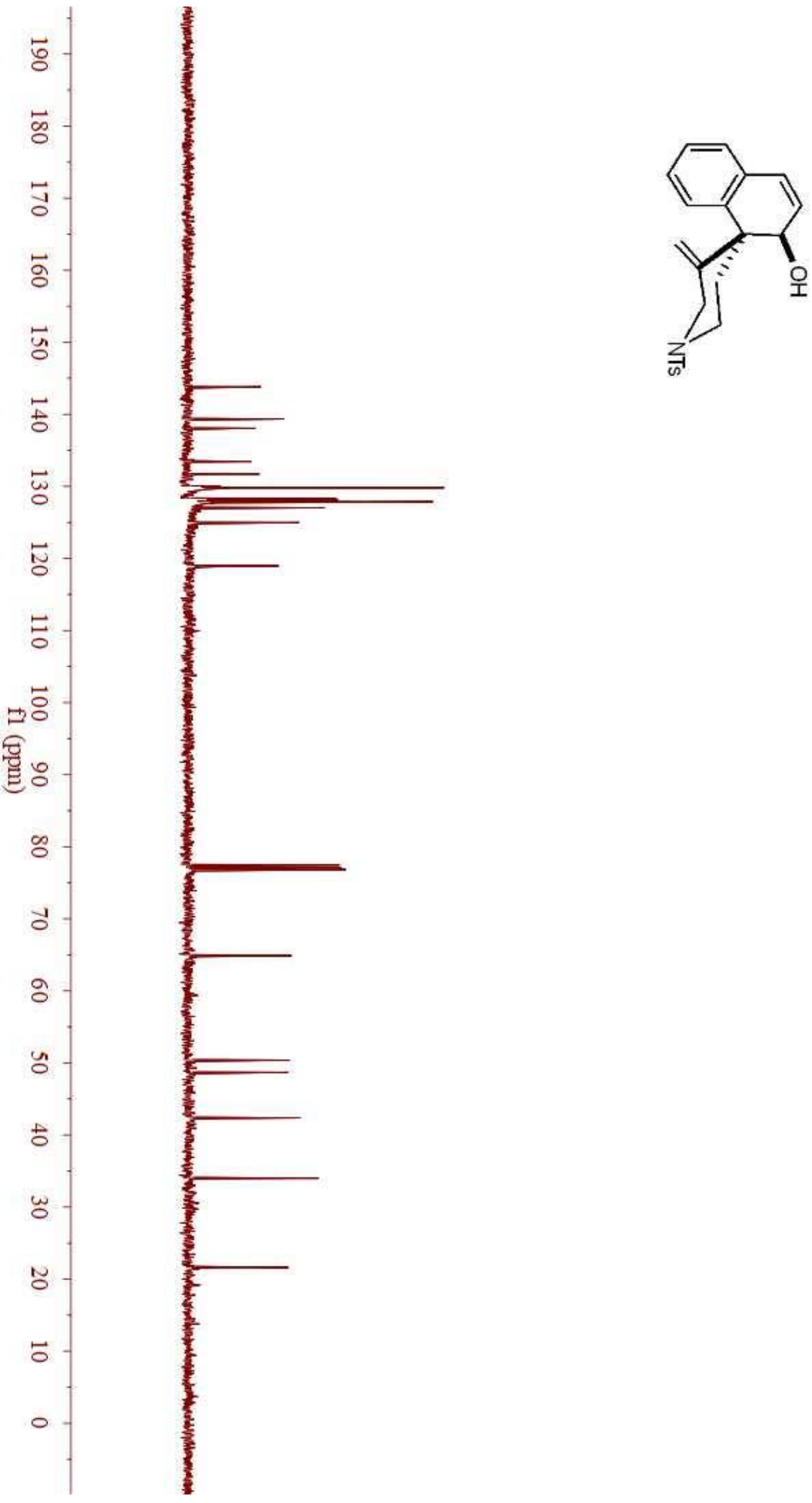

$\left[\begin{array}{l}143.800 \\ -139.358 \\ -138.074\end{array}\right.$

$-133,455$

- 131.724

129.942

- 129.819

$-128.325$

$-128.265$

$-128.132$

$-127.866$

$-127.044$

$-124.989$

- 118.974

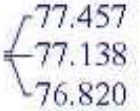

$-64.869$

$-50.383$

$-48.688$

$-42.381$

$-34.008$

$-21.627$ 
Figure $75^{1} \mathrm{H}$ and ${ }^{2} \mathrm{D}$ NMR spectra of $\mathbf{3 a}\left(400 \mathrm{MHz}, \mathrm{CDCl}_{3}\right)$

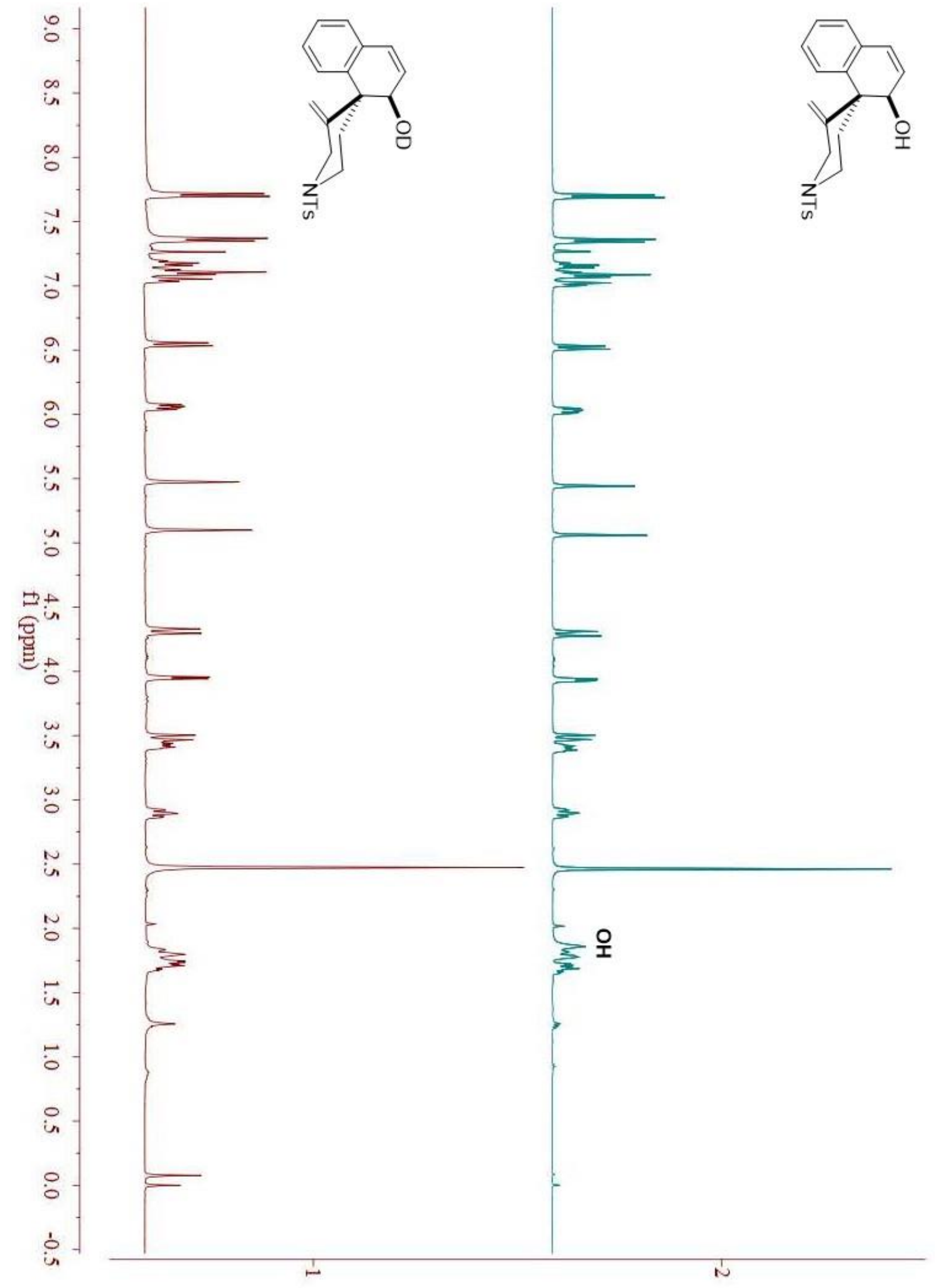


Figure $76 \mathrm{HSQC}$ NMR spectrum of $\mathbf{3 a}\left(400 \mathrm{MHz}, \mathrm{CDCl}_{3}\right)$

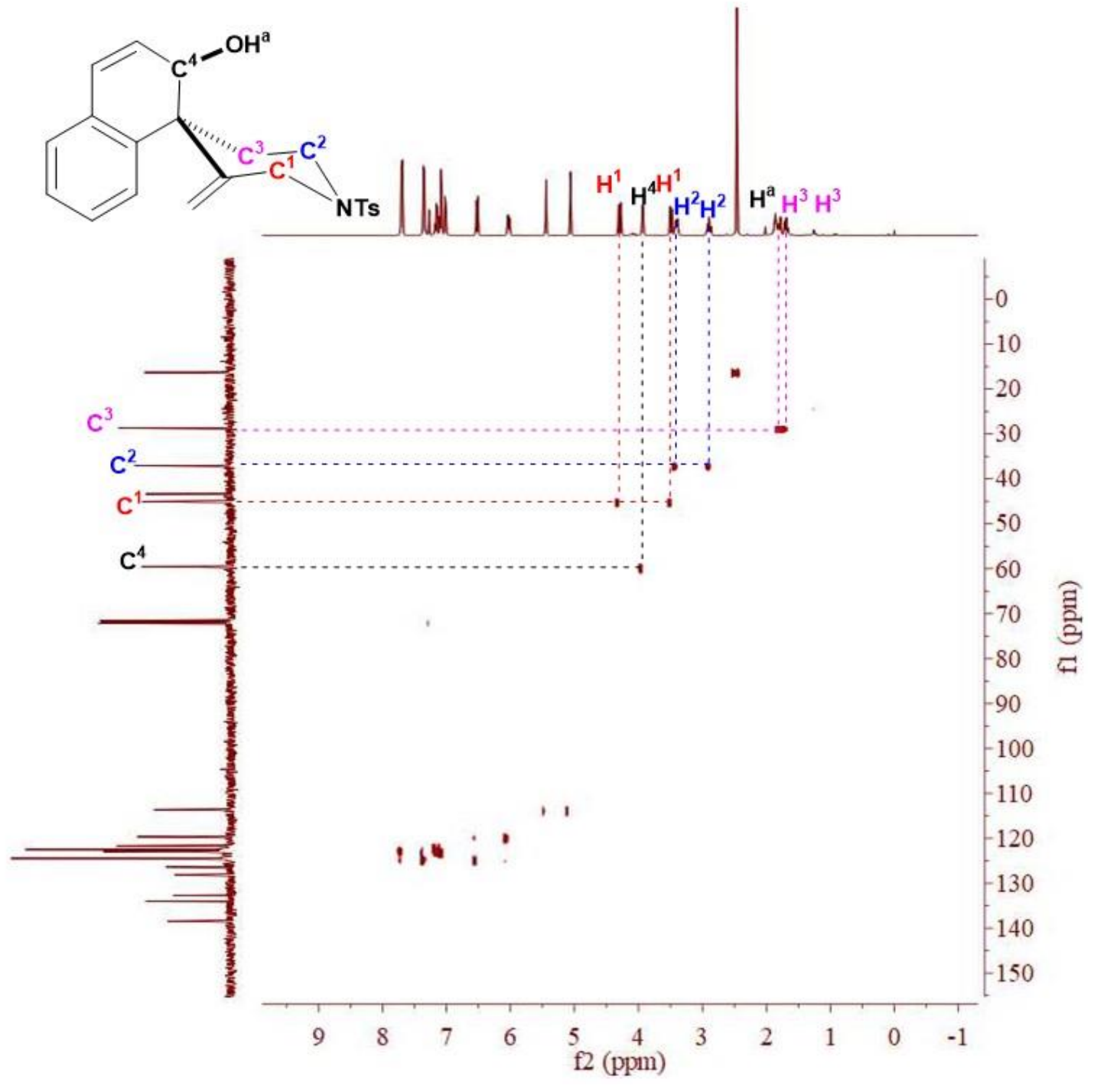


Figure 77 NOESY NMR spectrum of $\mathbf{3 a}\left(400 \mathrm{MHz}, \mathrm{CDCl}_{3}\right)$

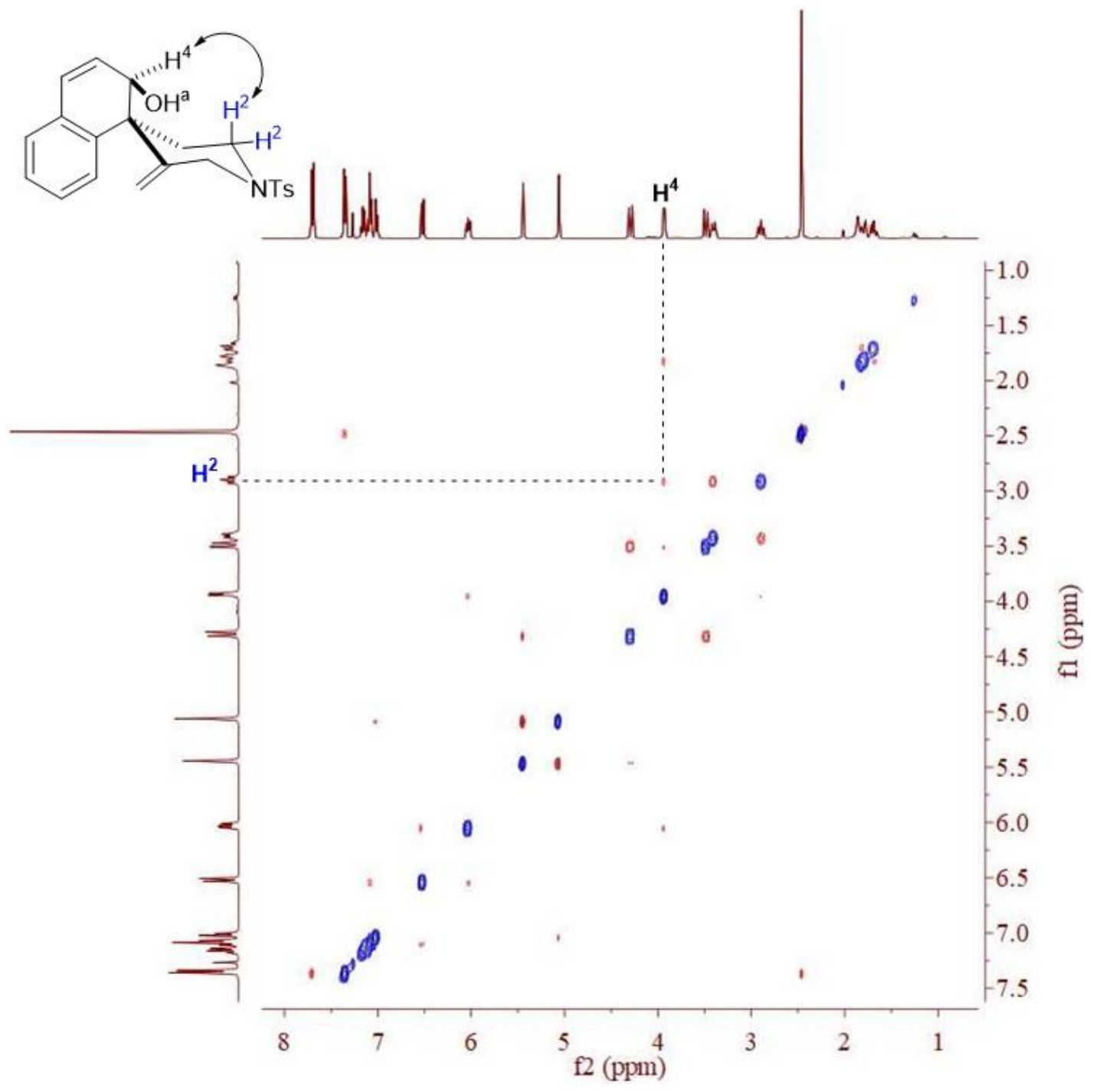


Figure $78{ }^{1} \mathrm{H}$ NMR spectrum of 3a' $\left(400 \mathrm{MHz}, \mathrm{CDCl}_{3}\right)$

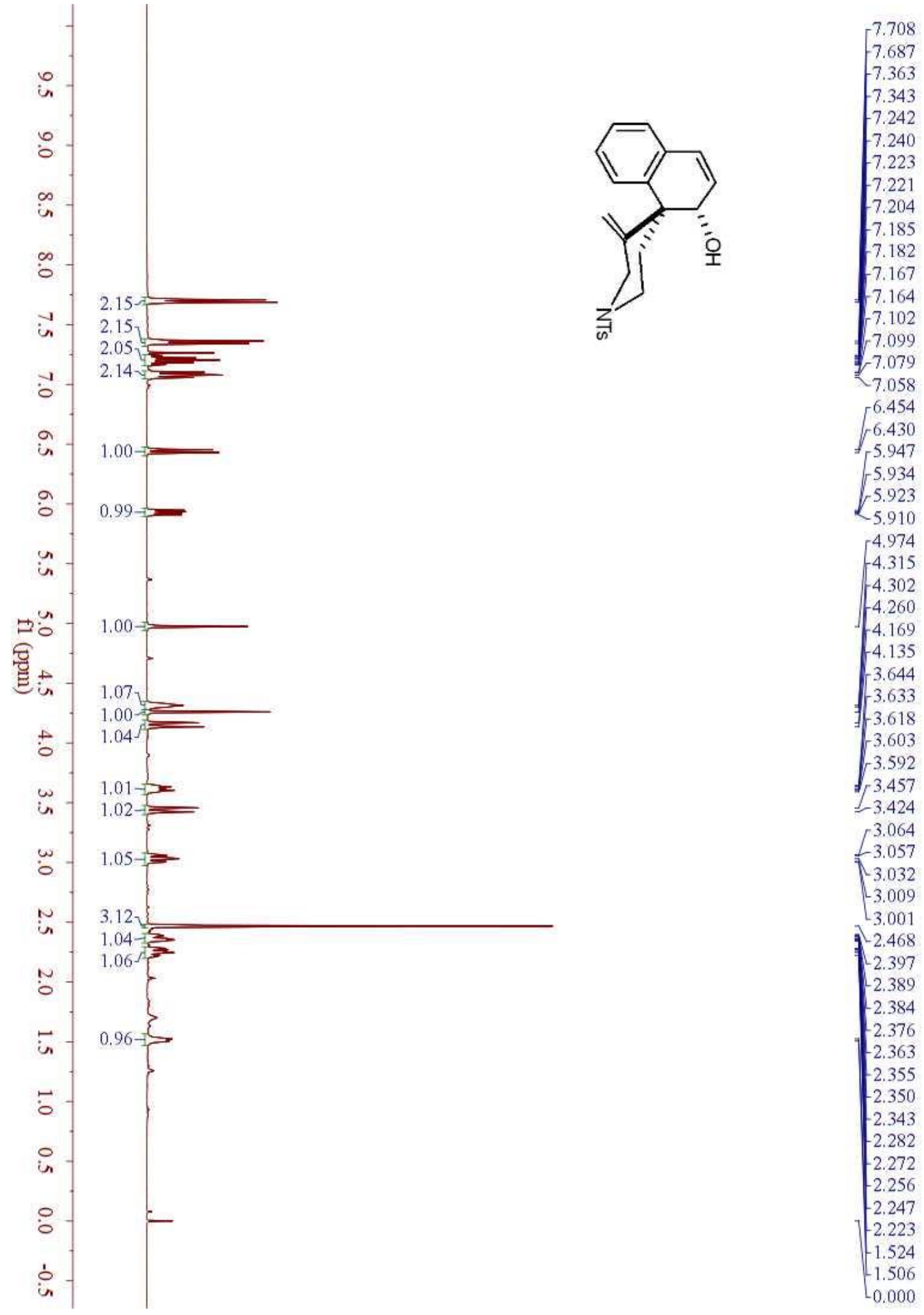


Figure $79{ }^{13} \mathrm{C}$ NMR spectrum of 3a' $\left(100 \mathrm{MHz}, \mathrm{CDCl}_{3}\right)$
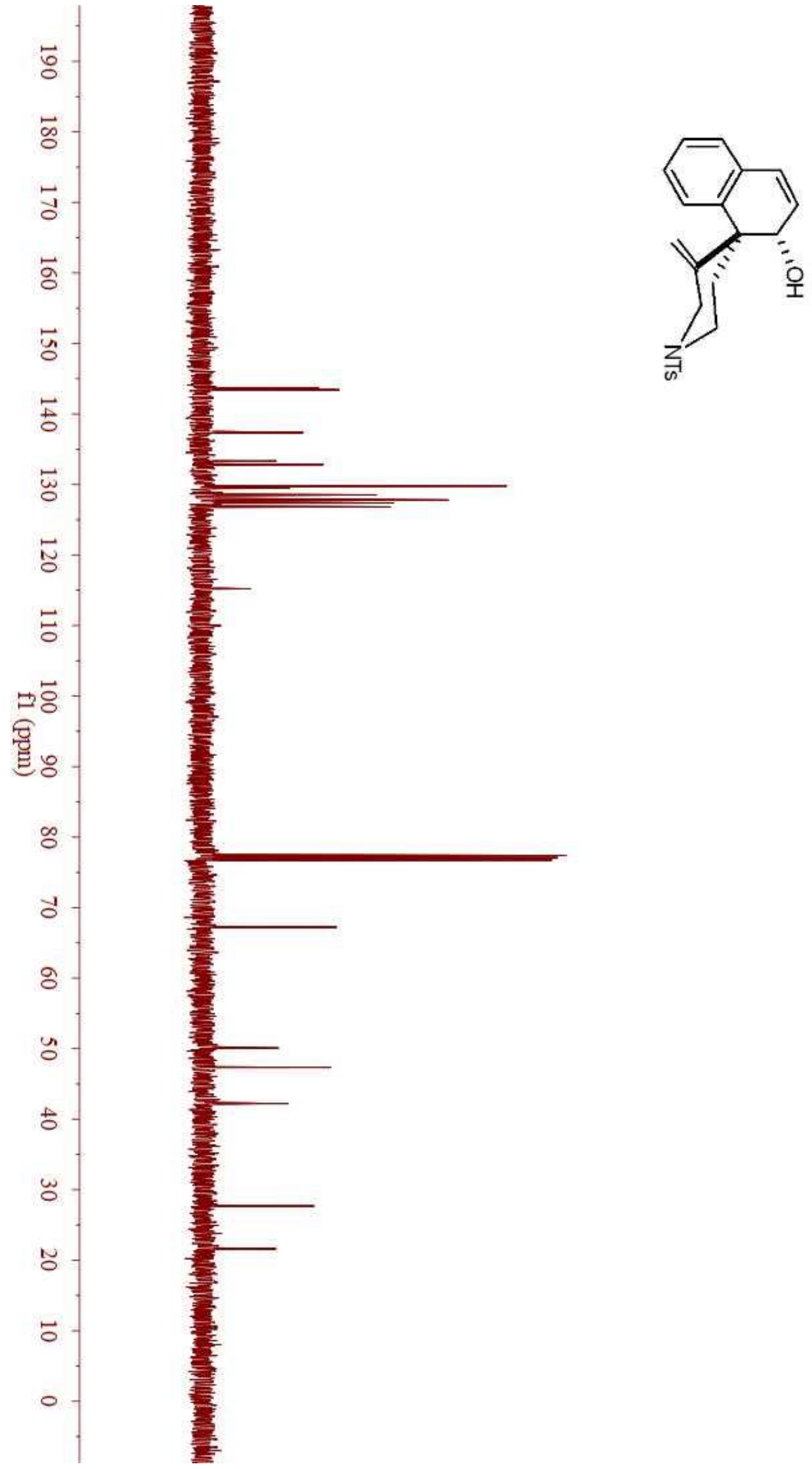

143.676

$-143.406$

$-137.344$

$-133.320$

$-132.835$

$-129.793$

$-129.476$

$-128.527$

127.793

$-127.460$

- 127.410

$-127.368$

$-126.831$

$-115.207$

77.397

$-77.079$

$-76.761$

$-67.224$

$-50.079$

$\checkmark-47.340$

$-42.208$

$-27.674$

$-21.610$ 
Figure $80{ }^{1} \mathrm{H}$ and ${ }^{2} \mathrm{D}$ NMR spectra of $\mathbf{3 a}{ }^{\prime}\left(400 \mathrm{MHz}, \mathrm{CDCl}_{3}\right)$

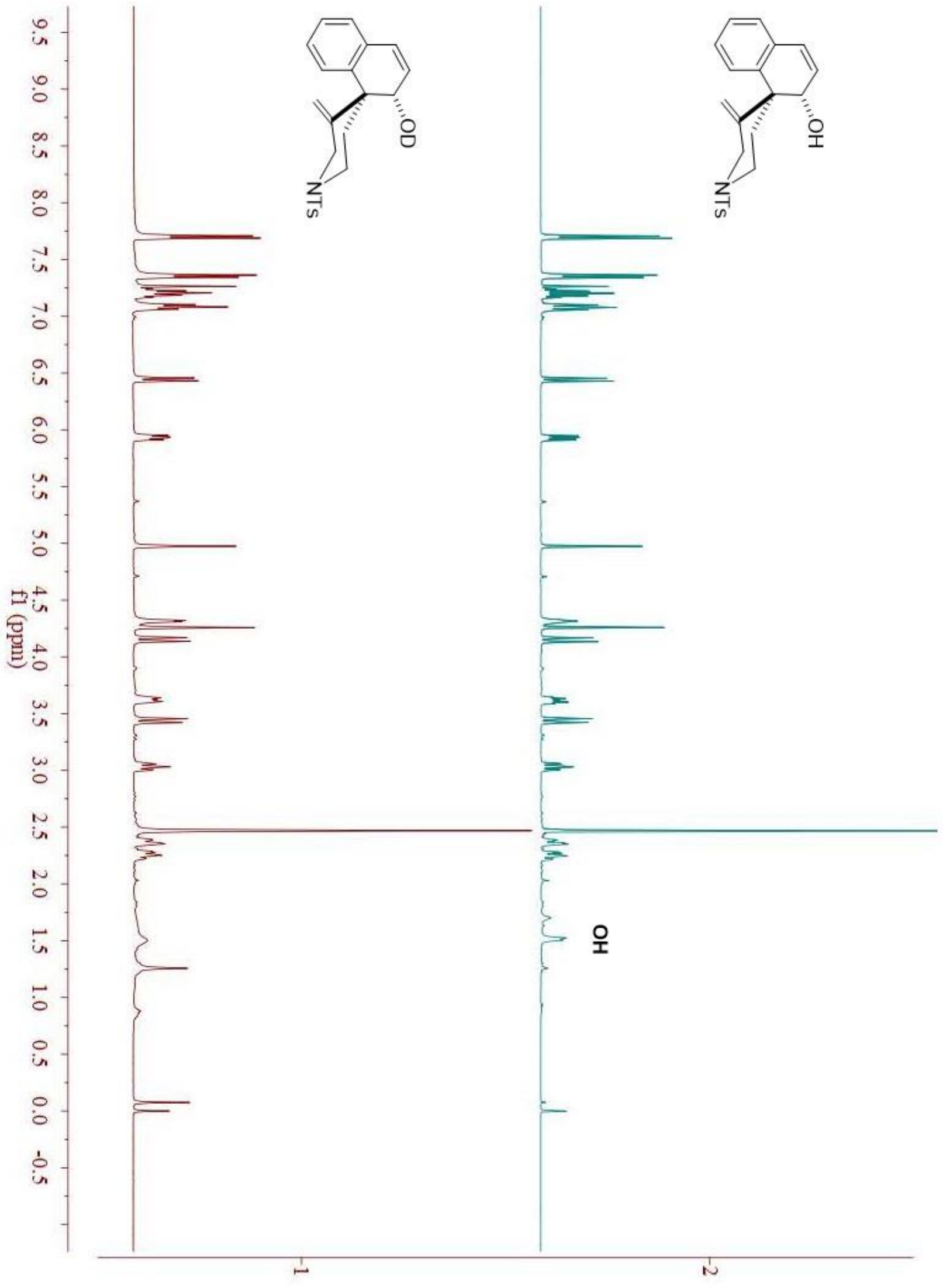


Figure 81 HSQC NMR spectrum of 3a' $\left(400 \mathrm{MHz}, \mathrm{CDCl}_{3}\right)$

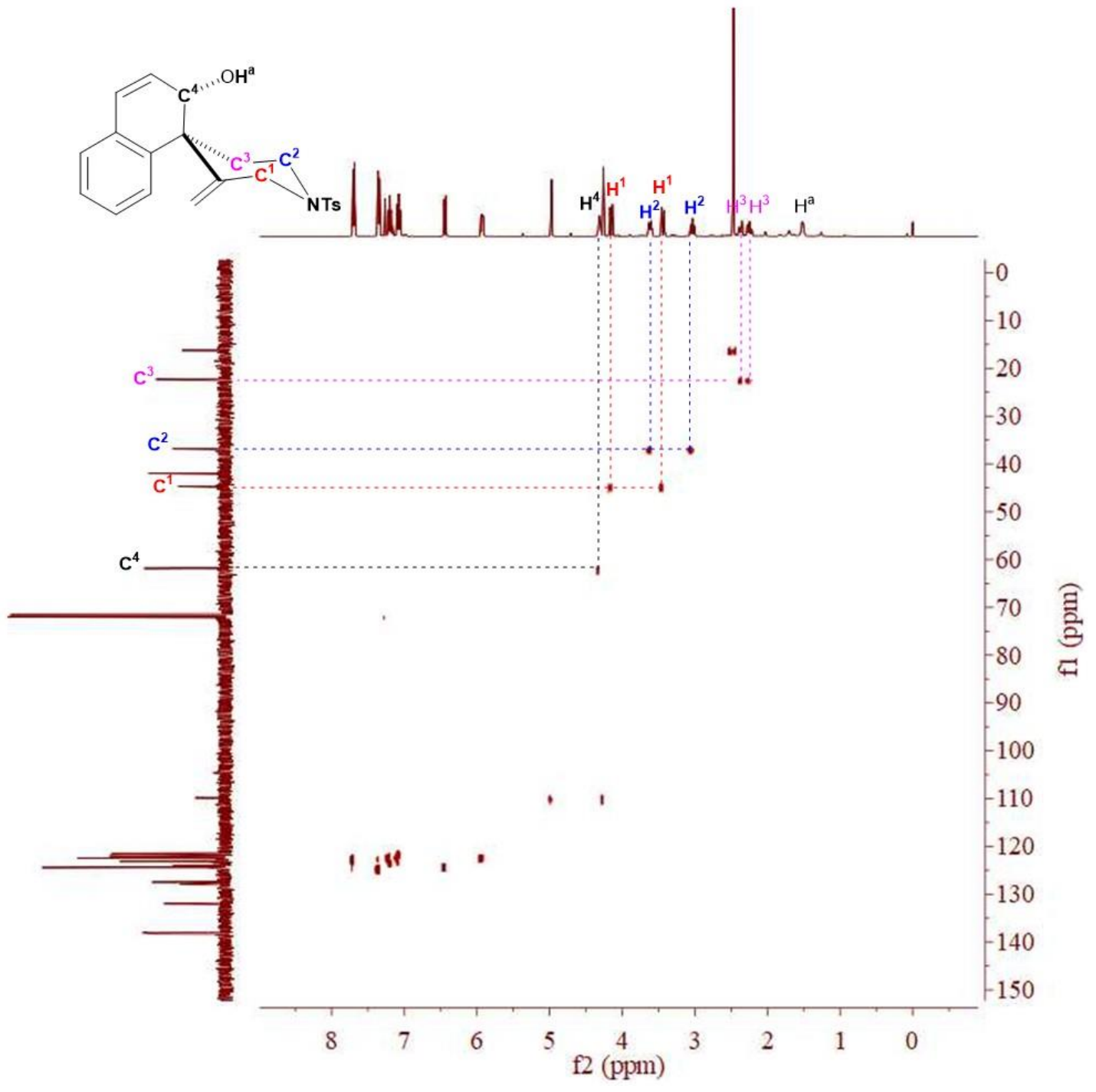


Figure 82 NOESY NMR spectrum of $\mathbf{3 a}{ }^{\prime}\left(400 \mathrm{MHz}, \mathrm{CDCl}_{3}\right)$

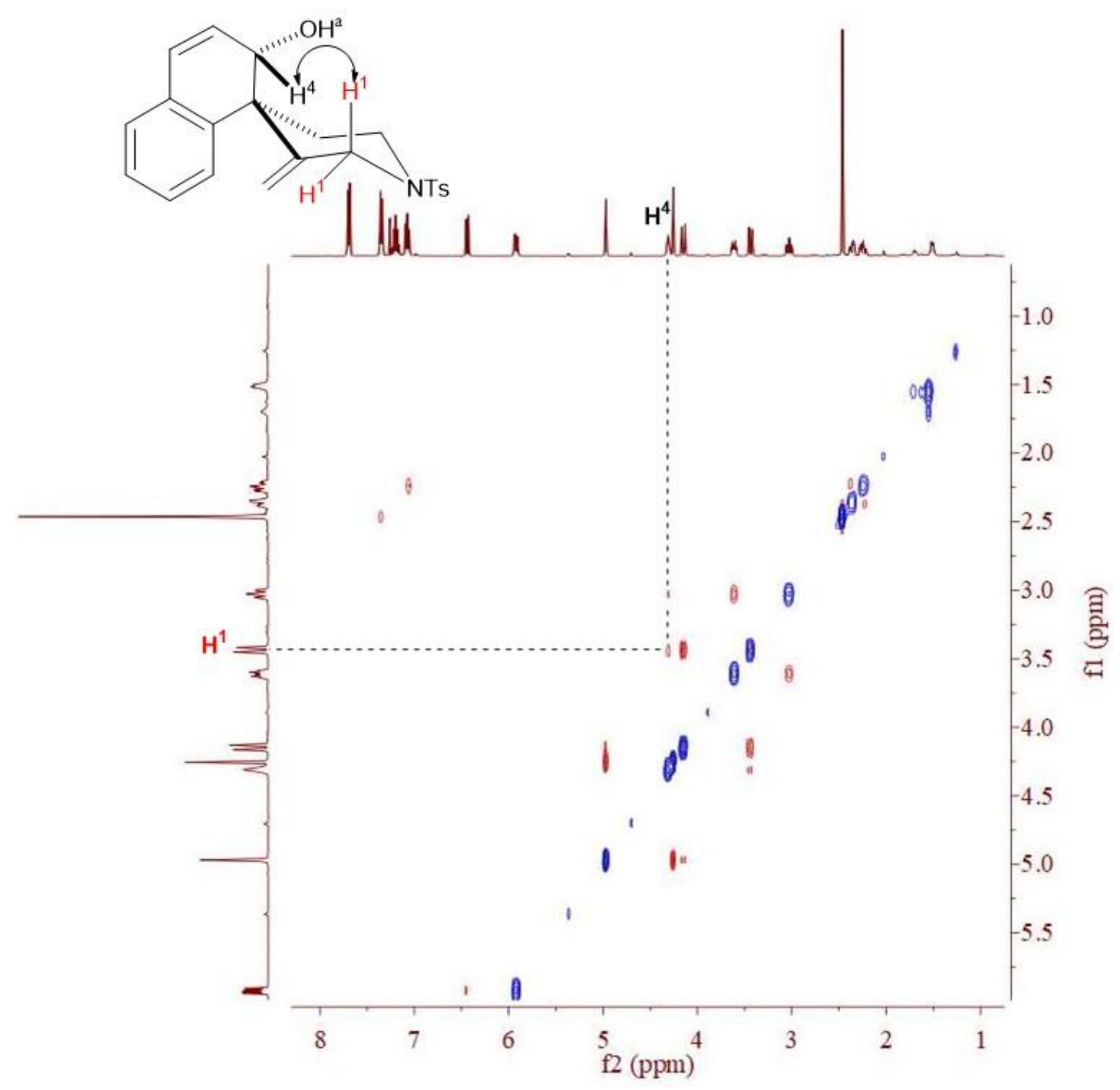


Figure $83{ }^{1} \mathrm{H}$ NMR spectrum of $3 \mathbf{e}\left(400 \mathrm{MHz}, \mathrm{CDCl}_{3}\right)$

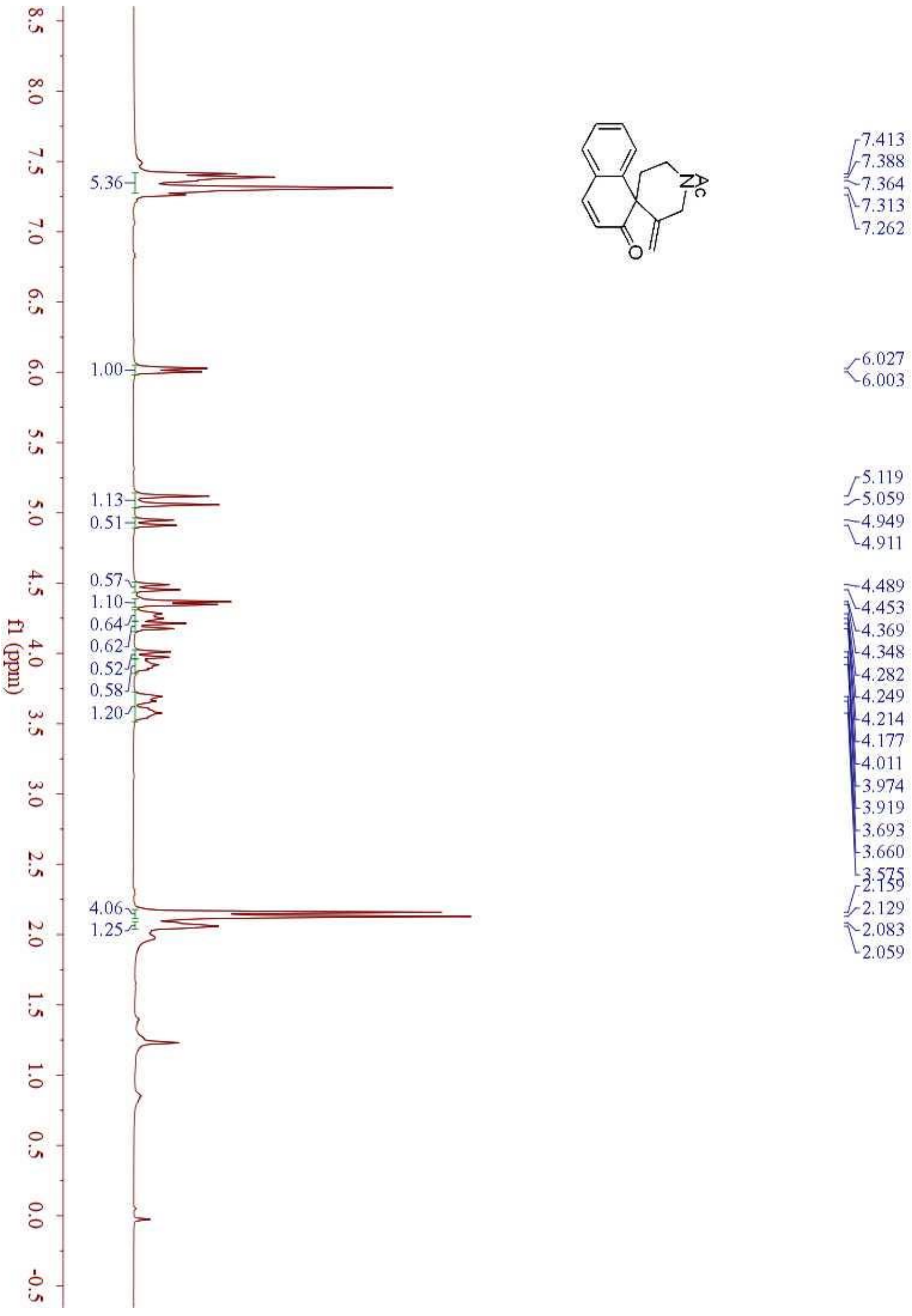


Figure $84{ }^{13} \mathrm{C}$ NMR spectrum of $3 \mathbf{e}\left(100 \mathrm{MHz}, \mathrm{CDCl}_{3}\right)$

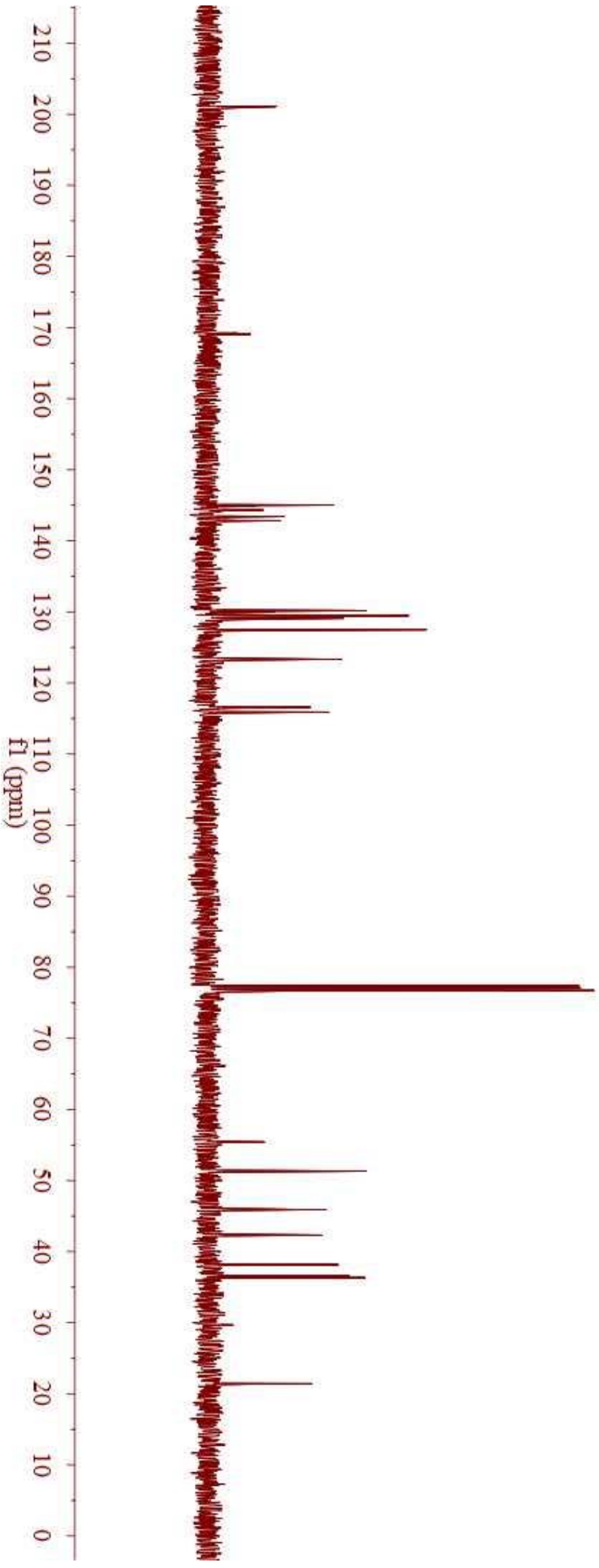

$-201.093$

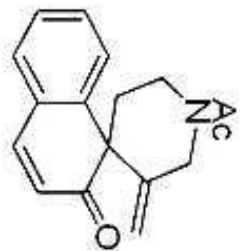

$-169.045$

$-145.027$

$-144.878$

$-144.429$

- 144.313

- 143.416

$-142.842$

130.195

$-130.167$

$-130.008$

$-129.95$

$-129.471$

$-129.360$

$-129.117$

$-127.501$

$-123.459$

$-123.341$

$-116.590$

$-115.908$

77.368

$-77.050$

$-76.732$

55.503

$-55.405$

$-51.323$

45.923

42.315

$-38.165$

$-36.591$

$-36.326$

21.570

21,406 
Figure $85{ }^{1} \mathrm{H}$ NMR spectrum of $\mathbf{3 e}\left(600 \mathrm{MHz}, d_{6}\right.$-DMSO)

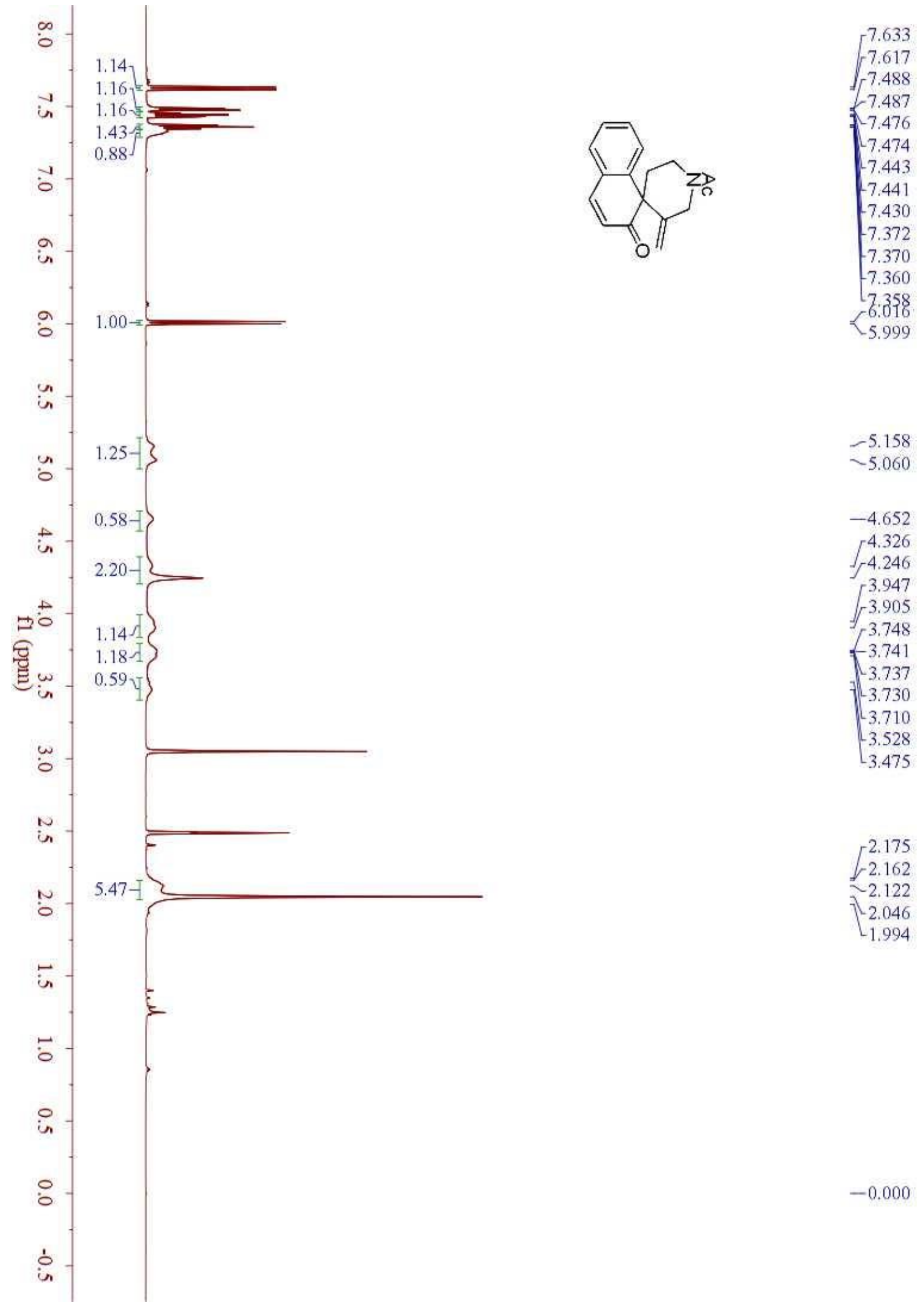


Figure $86{ }^{13} \mathrm{C}$ NMR spectrum of $3 \mathbf{e}\left(150 \mathrm{MHz}, d_{6}\right.$-DMSO)

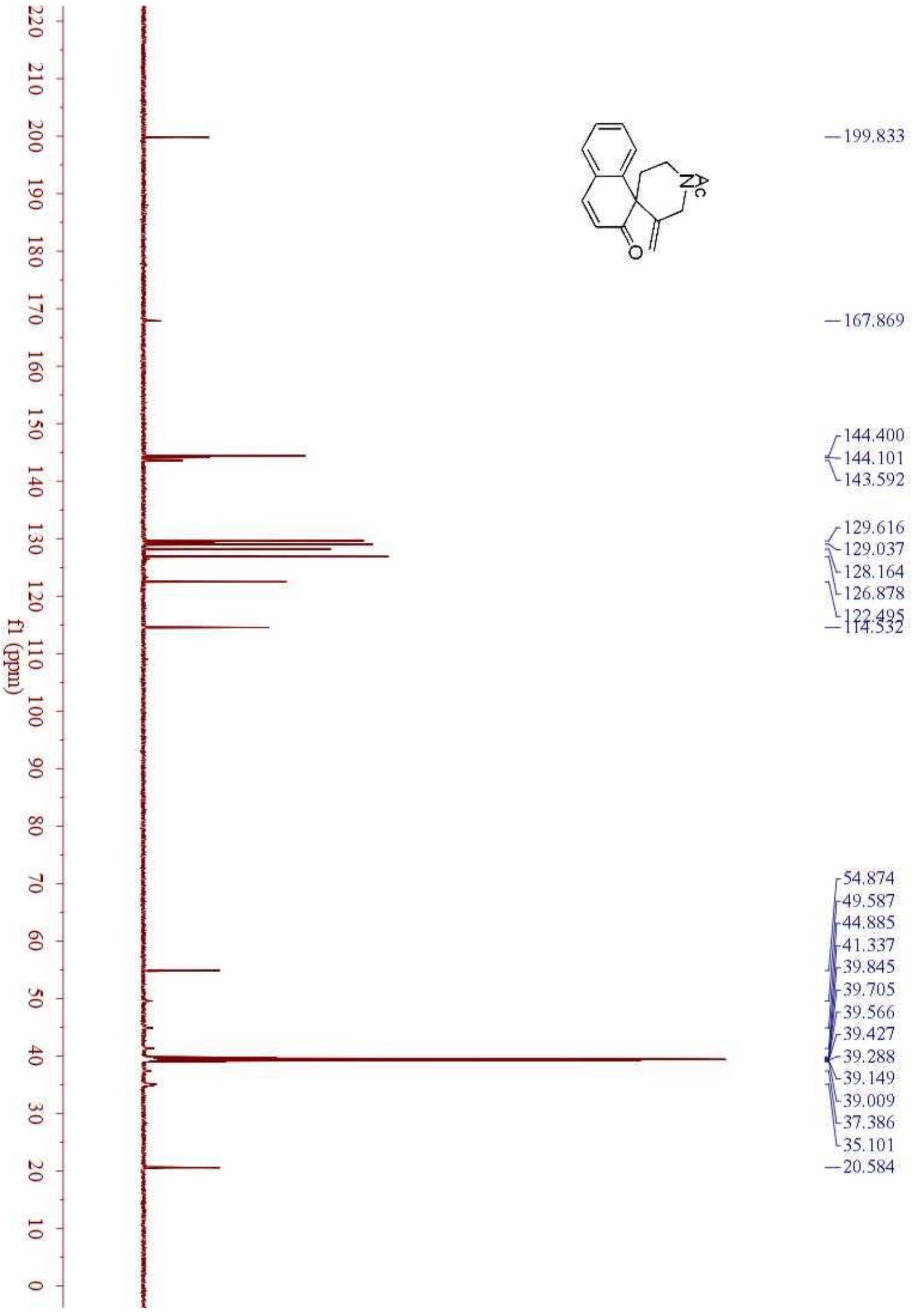


Figure 87 HPLC chromatograph of $\mathbf{2 a}$

\section{Empower2}

Multi Sample Summary

\begin{tabular}{|llll|}
\hline & \multicolumn{2}{c|}{ S A M P L E } & IN F O R M A T I O N \\
\hline \hline Sample Name: & wwt-14-67-rac-AD-H & Acquired By: & System \\
Sample Type: & Unknown & Sample Set Name: 1 \\
Vial: & $1: A, 1$ & Acq. Method Set: & wwt 9010254 \\
Injection \#: & 1 & Processing Method & fdfd \\
Injection Volume: & 10.00 ul & Channel Name: & W2489 ChA \\
Run Time: & 60.0 Minutes & Proc. Chnl. Descr.: & W2489 ChA 254nm \\
Date Acquired: & $11 / 12 / 2016$ 8:33:38 AM CST & & \\
Date Processed: & $6 / 28 / 202012: 10: 05$ PM CST & & \\
\hline
\end{tabular}

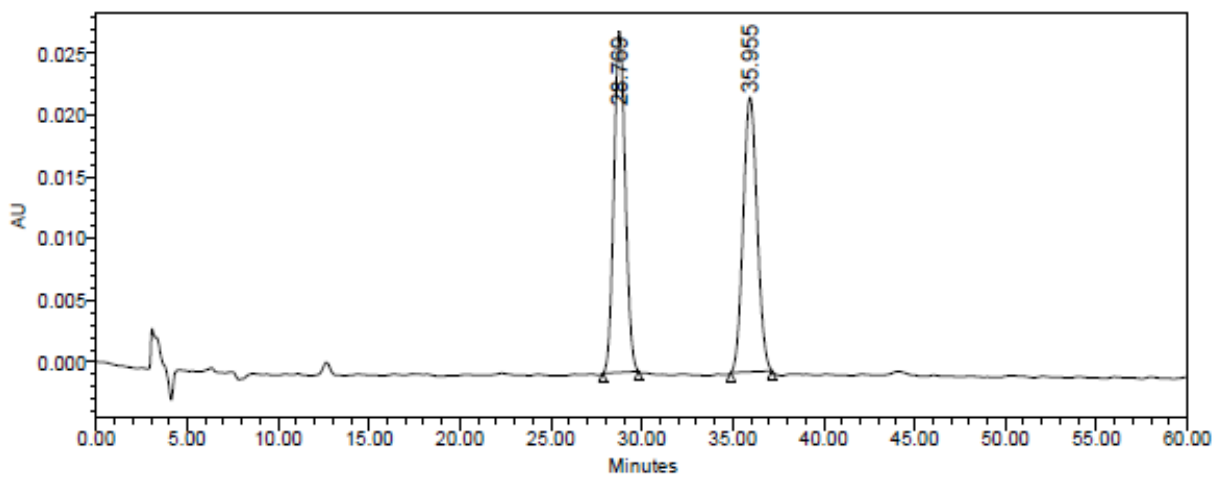

\begin{tabular}{|c|c|c|r|c|}
\hline & RT & Area & \% Area & Height \\
\hline 1 & 28.769 & 1200568 & 50.26 & 27633 \\
\hline 2 & 35.955 & 1187963 & 49.74 & 22246 \\
\hline
\end{tabular}

\section{Empower2}

Multi Sample Summary

\begin{tabular}{|llll|}
\hline \multicolumn{2}{c|}{ S A M P L E } & I N F O R M A T I O N \\
\hline \hline Sample Name: & wwt-15-35 & Acquired By: & System \\
Sample Type: & Unknown & Sample Set Name: & 1 \\
Vial: & 1:A,1 & Acq. Method Set: & wwt 9010254 \\
Injection \#: & 1 & Processing Method & rerer \\
Injection Volume: & 10.00 ul & Channel Name: & W2489 ChA \\
Run Time: & 50.0 Minutes & Proc. Chnl. Descr.: & W2489 ChA 254nm \\
& & \\
Date Acquired: & 1/5/2017 8:46:47 PM CST & & \\
Date Processed: & $6 / 28 / 2020$ 12:11:58 PM CST & & \\
\hline
\end{tabular}

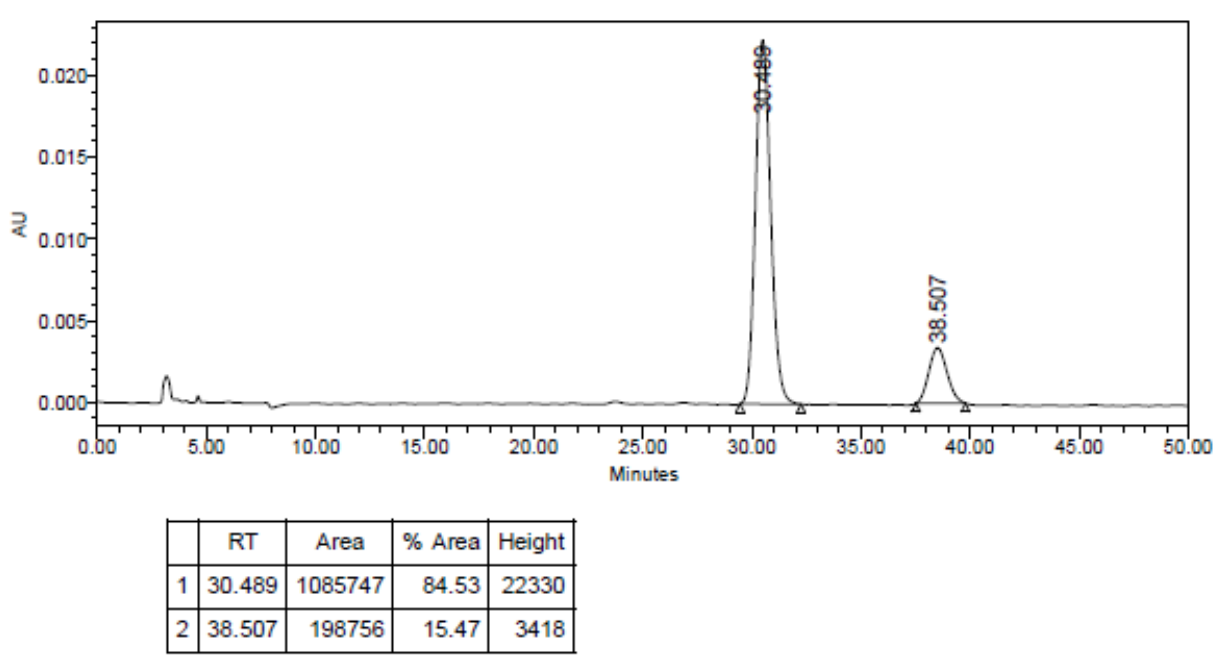

